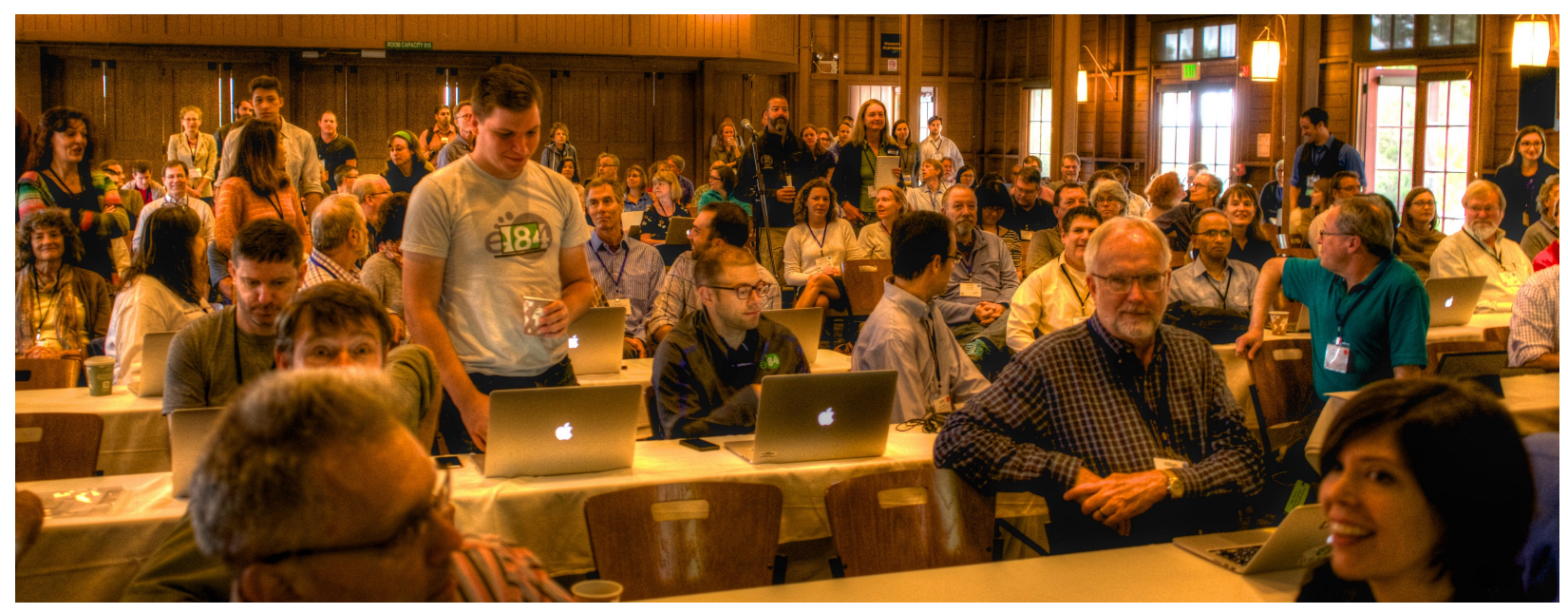

\title{
Open Scientist Handbook
}

\section{Bruce R. Caron}

New Media Research Institute, Santa Barbara, and the Ronin Institute

A sharable version of the Bibliography is on the web HERE

License: $\underline{\text { Creative Commons Attribution 4.0 International License (CC-BY 4.0) }}$

All photographs, unless credited with their own license, are the author's or public domain (CCO). 


\section{Acknowledgements}

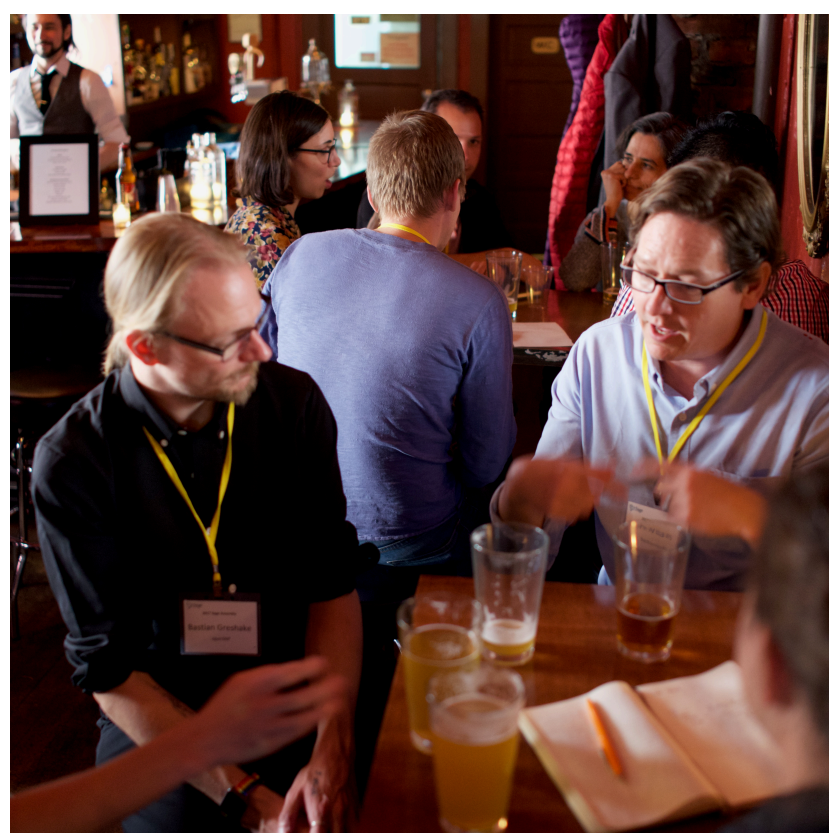

I have to thank an army of open-science nerds out there working to build Science 2.0 over the past 20 years. The list of individual names would be long, and some would be missing, so I thank all of you. I've had more cerebration (mind-opening conversation) events than I can count, with some of the kindest people on the planet. With thanks to all the organizations and foundations that are supporting the transition to open. As many of your ideas as I could, I have included in this work. I have borrowed these and reused them as they are meant to be reused. You are free to borrow anything you find in here that you can use. Thank you Alfred P. Sloan Foundation, in particular, for funding the scholarly commons workshop.

Contributors on this manuscript revisions:

As this work grows and changes, others have stepped up to add their insights and critiques. Dr. Kennan Salinero added several insights, and worked on the many typos I've missed. 


\section{Contents}

$\begin{array}{ll}\text { Foreword } & 5\end{array}$

$\begin{array}{ll}\text { The Open Scientist as a Culture Change Agent } & 7\end{array}$

A guide for scientists to become culture change agents for open science 16

The Work of Culture in Your Open Science Organization 20

Performing Open Science Culture $\quad 28$

Values, freedoms, and principles of Open Science $\quad 34$

Kindness, Culture, and Caring: The Open Science Way $\quad 40$

The Onlyness of the Career Open Scientist 45

10 things every open-science culture-change agent needs to know about. 53

$\begin{array}{ll}\text { Talking Virtue and Values in Open Science } & 60\end{array}$

Fierce Equality $\quad 66$

It's time for science to admit that no scientist is a lone giant in their field 75

$\begin{array}{ll}\text { Demand Sharing: a Real Sharing Economy for the Academy } & 81\end{array}$

Building a gift economy: the dance of open science culture 88

As an open scientist, you're good-to-get what you need 98

$\begin{array}{ll}\text { Demand sharing and the power of pull } & 107\end{array}$

$\begin{array}{ll}\text { A logic of abundance } & 112\end{array}$

$\begin{array}{lr}\text { Idea Farming } & 120\end{array}$

$\begin{array}{ll}\text { Against Patents in the Academy } & 130\end{array}$

Is My Learned Society Obsolete? 139

Open Science Badges are Coming-and the New Nobel 143

$\begin{array}{ll}\text { The Infinite Play of Science } & 154\end{array}$

$\begin{array}{ll}\text { Learning the infinite play of science } & 168\end{array}$

$\begin{array}{ll}\text { The practical wisdom of doing science } & 175\end{array}$

$\begin{array}{ll}\text { Knowing and conversation in the academy } & 180\end{array}$ 
Joy, fun, and love

$\begin{array}{ll}\text { Commons are the future homes for science } & 197\end{array}$

$\begin{array}{ll}\text { Open Science needs Online Organizations } & 218\end{array}$

Double-loop Governance is the launchpad for open science collaboration 221

Open Collaboration Networks $\quad 231$

$\begin{array}{ll}\text { Open Collaboration Networks are the future of science } & 241\end{array}$

Workshops and beyond $\quad 253$

$\begin{array}{ll}\text { Open science heals toxic culture } & 260\end{array}$

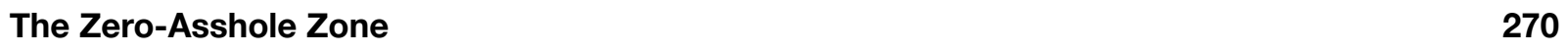

The Congruent Scientist: Infinite play builds personal wisdom 276

$\begin{array}{lr}\text { Bibliography } & 289\end{array}$ 


\section{Foreword}

\section{How to use this handbook}

"The shift is to implement, at all levels in each organization, a bias toward action" (Nowviskie 2012; <http://nowviskie.org/2012/lazy-consensus/> Accessed June 30, 2019).

The Open Scientist Handbook is designed to give any scientist on the planet the know-how and tools to become an effect open science culture change agent at your job, in your professional organizations and collegial associations, and in your personal life. "Open science" - what people after 2030 will call "science" - refactors $20^{\text {th }}$ Century science cultures to restore those practices, motivations, virtues, rigor, and joys that have long been the incentives for smart, creative individuals like you to challenge the universe's unknowns as a scientist, instead of devising clever derivative financial devices for Wall Street (which you totally could have done).

Open scientists take full advantage of emergent technologies (e.g., the internet, cloud computing, online networking) in order to build shared research repositories and platforms that provide abundant, mineable data, reproducible experiments, lateral learning for new methods, rapid research publication with rigorous review, streamlined and fair funding opportunities, and world-wide knowledge access with equal participation.

Your future is better with open science. Why is that? Open science offers new value to your work and your science life. Open science multiplies your research's impact. When you add your research objects (from ideas to findings) to open repositories, these can be rapidly discovered, evaluated, shared, and applauded; and all of this without being subjected to arbitrary metrics that institutions have gamified for their own purposes (e.g., journal impact factors), instead of providing value to your own work. As open science is grounded on Demand Sharing and Fierce Equality, you can also pull resources from the common pool to accelerate your work, and discover new collaborators across the planet.

This vision of an open, global science endeavor confronts a range of entrenched institutional practices and perverse incentives: a toxic culture that has hobbled science for decades. Open scientists need the know-how and tools to tear down these practices and to interrogate these incentives, in order to replace them.

Culture is a problematic term, not just for the natural scientist trying to wrap her thoughts around its many uses, but also within anthropology and sociology where it has more of a home. Culture is also at the core of the entire endeavor we call "open science." This handbook will provide a usable working description of "culture"-a handy culture monkeywrench-as this applies within the social arenas wherever scientists operate: from 
academic departments, to funding-agency proposal review panels, to professional associations; and also to their personal choices.

Many academics are familiar with projects that work to build science "infrastructure" using shared technology platforms and standards. The twin task of this is to build social infraculture using shared principles, norms, and governance logics. With this guidebook you can confidently announce that you are ready to tackle culture change, armed with real knowledge of what academic culture is, how it works (and where it doesn't), and what to do about it.

Use this handbook as guidance on how you can become an open science culture change agent in your life and your work. As a handbook, you can use this as a guide, dipping into its contents as you need, without reading the whole thing cover-to-cover. Do you want to know a bit about "culture" or "community"? You can do that here. Heard about "the commons" and need some real information? There's a chapter for that. Dealing with an asshole-withtenure? This book can help. Everything you need to begin your own conversations about open science in your lab, your university, or your learned society can be found here. Do you work in a funding agency or foundation? This book will help you focus your funding strategies.

This handbook's guidance does not require that you adhere to some single mode of thought or action; how you articulate your own position on open science is entirely up to you.

"Physics is to mathematics what sex is to masturbation" (Richard Feynman, in Krause and March 1994).

... "Open science is to science what sex is to masturbation" (everybody in 2022). 


\section{The Open Scientist as a Culture Change Agent}

\section{You do science. Do you know science?}

There are books and libraries of books that talk about science: its history, sociology, philosophy, politics, and practice. As a scientist, you've likely gotten this far in life without reading any of these. You probably don't need to start now. In this essay, a few remarks about science will help anchor the Open Scientist Handbook into a particular framework for science as a project, as an endeavor, and a life-way. You are already a scientist, so you don't need a general introduction to "science." Also, you can learn everything you need about open science as a practice by checking out the (Open Science MOOC; Accessed May 28, 2020).

This essay links into several other essays in the book that you can explore if you wish, when it's convenient. Here you will find several Richard Feynman quotes. Do you want a good example of an open scientist? Be like Richard Feynman (who died before open science became a meme):

"If it turns out it's like an onion with millions of layers and we're just sick and tired of looking at the layers, then that's the way it is, but whatever way it comes out, its nature is there and she's going to come out the way she is, and therefore when we go to investigate it we shouldn't pre-decide what it is we're trying to do except to try to find out more about it" (Feynman 2005).

\section{Science is infinite because nature demands infinite play.}

Nature is not entirely knowable; for very good reasons, including its emergent, adaptive complexity, and our embedded place within it (cf. Max Planck 1932/2015). Not yet knowing everything about nature and the universe is why science still exists. Nature not ever being knowable is the scientist's best job security.

Nature is a great part of what James P. Carse (1987) called the "infinite game." By studying nature, scientists get to be players in/with this infinite endeavor. Not many humans get to do this for a living, but all of us do this because we are alive. When we stop breathing, the infinite playing goes on without us.

Carse has a list of distinctions between "finite" and "infinite" games. Francis Kane's New York Times (04/12/1987) review of Carse's book says:

"Finite games are those instrumental activities - from sports to politics to wars - in which the participants obey rules, recognize boundaries and announce winners and losers. The infinite game - there is only one - includes any authentic interaction, from touching to culture, that changes rules, plays with boundaries 
and exists solely for the purpose of continuing the game. A finite player seeks power; the infinite one displays self-sufficient strength. Finite games are theatrical, necessitating an audience; infinite ones are dramatic, involving participants."

\section{Finite games and infinite play}

The Handbook has a quibble about Carse's use of the term "game" for what is really just infinite play: in this case, "science play." Using the word "game" to describe an event with constantly changing rules and boundaries, where anyone can play and the more the better, and where the play never ends; this twists all meanings of the word "game" into unrecognizable knots. We all know what games are. They are defined by fixed rules, starting and ending points and, in many cases, specified boundaries. We play in them, and play at them, but they have real structures and definite rules that must be followed for the game to continue. Like infinite play, finite games are open-ended: their result is a future event. Unlike finite games, infinite play is neverending.

So the Handbook will use "game" for all the "finite" cases, where this matches every usual sense of the word game, and "play" for the infinite form. This echoes the older use the word "play" to signal skill and creativity. Learning to play is a common feature of cultures: play the violin, play the market. There is even foreplay (real open scientists excel at this). "Word play" gives us poetry. "Sword play" gives us corpses. "Science play" gives us knowledge of the universe. Infinite play includes breathing in and out until we can no longer do this. It's a biological impulse to keep playing, understanding that when one must finally stop, others will continue.

The point of infinite science play is to keep playing science, to learn how to play better, and to add players to the mix; to sustain the play and the knowledge required to play at the highest levels; to change the rules, not to cheat, but to explore. In infinite play, your strengths are not what you are good at, but what you every so much want to get better at. That's why you jumped head first into science.

Infinite play goes on even when humans are distracted by the finite games they make up to give themselves victories to distinguish their efforts. The academy can choose to invest in science play, or it can get distracted by finite games of manufactured scarcity, ersatz excellence, and cumulative advantage. This is where we are and the choice we need to consider.

Because nature is intimate within infinite play, science cannot avoid playing along. Biological evolution, for example, is a theory that describes some of the adaptive and emergent possibilities of infinite play. There is no end-point to evolution; no species really 
wins, some of them just have the chance to keep on playing. In fact, species extinction has a general positive effect on the robustness of the ecosystem.

There is also some discussion in the Handbook for "play," as we know this from childhood. You can look ahead to The practical wisdom of doing science to find out how much of the wisdom you might bring to your life you learned playing as a child. Other sections include the emotional tone of doing science (Joy, Fun, and Love), which looking into playfulness as an attribute of scientific discovery. You might not be having fun yet, but if open science blossoms, who knows?

\section{Complexity theories for the academy}

Infinite play is an intrinsically complex knowledge-management endeavor. Recent organizational management theories, such as the Cynefin Framework (Accessed May 28, 2020) started at IBM, warn that there are no "best practices" to deal with the "wicked problems" of adaptive complexity. This warning includes not just the marketplace, but also nature and culture. It turns out we are surrounded by emergent forces, and $20^{\text {th }}$ Century management techniques are not up to the task. Open science mines the latest complexity theories to help guide its path.

While science methods have been addressing nature's complexity for centuries, science knowledge-management and organizational governance have not kept up. It's not hard to imagine science as an early-enlightenment project housed in late-medieval organizations. Open science looks to bring science governance and practice into the $21^{\text {st }}$ Century.

Nature is fantastically more complex, ambiguous (despite the claims of "natural laws"), and emergent than is our ability to understand this. Here is the asymmetry that makes science infinite play, a story instead of a recipe. The amount of knowing that happens with any new science discovery is an order of magnitude greater than the description of this "finding" in a scholarly paper. That's why any new finding is worth a thousand conversations. The record of the finding is only the scat: the knowing is the actual beast, and needs sharing.

The asymmetry of "knowing" in a room at a science conference is enormous. The room knows orders of magnitude more about the topic than does the speaker. The larger the conference, the more this unspoken knowing - this room silenced, listening to a speakeris wasted at the event. We never need to go back to 10,000 person science meetings. Better to have five hundred small conversations, with an online platform to capture these.

\section{No governance here. But soon.}

This is an essay on science, not governance. Many of the sections of the Handbook offer governance guidance. Here it is only important to relate a couple major ideas. 
- First: your organization's governance needs to support science play. If your department, university, or research lab is still talking about "excellence," or "we are ranked \# X!," or "the average salary of our graduates is $\mathrm{Y} \$$," you are still playing finite games.

- Second: organizations that play finite games against others that use infinite play will always lose. Infinite play is a “long game." Its players don't care what other organizations are doing. They play to get better, not to win. Over time, they will outinnovate, out-think, and out-knowledge any peer who is chasing short-term finite wins.

- Third: science is already positioned for infinite play; it gets funding from society (science goods are public goods); it holds a long-term privileged status within society; its "foe" (nature) is formidable and pushes science to ever greater tasks; its plan is flexible, it will reinvent itself as needed; its goal is just and grand: sharable knowledge of the universe.

To join into infinite play, however, science, and your workplace, needs one more thing: it needs you, and others like you, to step up and lead.

Science has never been winnable. Nobody gets to figure everything out and finish science. Every bit of new knowledge is inextricably bound with a whole lot of other bits. It is a great example of the "long game." Likewise, any bit of learning, every insightful thought or sentence delivered in your lecture, is fully dependent on a history filled with a whole lot of other learning moments: all of which are equally fallible.

\section{Science wallows in doubt, devours unknowns, and excretes bits of incomplete knowledge}

"When Socrates taught his students, he didn't try to stuff them full of knowledge. Instead, he sought to fill them with aporia: with a sense of doubt, perplexity, and awe in the face of the complexity and contradictions of the world. If we are unable to embrace our fallibility, we lose out on that kind of doubt" (Schultz 2011).

Science looks squarely into the unknown. A scientist is never as interested in the work she has already published as she is in the next unknown she is tackling in her research. Science's knowledge-mignardises (or petit fours: sounds better than excrement) can and have accumulated into important and useful—but still incomplete-facts and theories about our world and ourselves. And only science can do this.

"There are a lot of facts to be known in order to be a professional anythinglawyer, doctor, engineer, accountant, teacher. But with science there is one important difference. The facts serve mainly to access the ignorance.... You use those facts to frame a new question - to speculate about a new black cat. In other words, scientists don't concentrate on what they know, which is considerable but 
also miniscule [sic], but rather on what they don't know. The one big fact is that science traffics in ignorance, cultivates it, and is driven by it. Mucking about in the unknown is an adventure; doing it for a living is something most scientists consider a privilege (Firestein 2012).

Science is a "world-building" exercise; it strives to explain every-thing it contacts. There are strands of complementary knowledges or untested theoretics that could use some investigation, there are "pseudo-sciences" like Astrology, but there is no "alt-science" world. The placebo effect shows we have a lot to learn about the healing process, but does not invalidate what we know.

The main adversary to science is bad science; open science looks to remove the (perverse) incentives behind most of today's shaky research methods and results:

"[I]n science... it is precisely when people work with no goal other than that of attracting a better job, or getting tenure or higher rank, that one finds specious and trivial research, not contributions to knowledge. When there is a marked competition for jobs and money, when such supposedly secondary goals become primary, more and more scientists will be pulled into the race to hurry 'original' work into print, no matter how extraneous to the wider goals of the community" (Hyde 2009).

Science rests on the possibility that everything it knows today is wrong. As Feynman noted: "Once you start doubting, just like you're supposed to doubt, you ask me if the science is true. You say no, we don't know what's true, we're trying to find out and everything is possibly wrong" (2005). Kathryn Schultz wrote an entire book on Being Wrong; science has a central spot in this work:

"In fact, not only can any given theory be proven wrong... sooner or later, it probably will be. And when it is, the occasion will mark the success of science, not its failure. This was the pivotal insight of the Scientific Revolution: that the advancement of knowledge depends on current theories collapsing in the face of new insights and discoveries. In this model of progress, errors do not lead us away from the truth. Instead, they edge us incrementally toward it" (Schultz 2011).

Science makes no claim to be right, but every claim to be the go-to method that can find out if something is wrong. From there, it harvests knowledge that has not (yet) been shown to be wrong; this is as close to being right/true as there is. And scientists get to have fun by being less-wrong today than yesterday. Scientists are passionate knowledge explorers. 


\section{The joy of discovery needs a home in the center of science}

"Another value of science is the fun called intellectual enjoyment which some people get from reading and learning and thinking about it, and which others get from working in it. This is a very real and important point and one which is not considered enough by those who tell us it is our social responsibility to reflect on the impact of science on society" (Feynman 2005).

Science is hard. It is the hardest ongoing task in all of humanity: after child rearing. One might expect society to honor, celebrate, and reward scientists for their labor.

For now, just consider that time spent doing infinite play can be intrinsically rewarding. In fact, it is potentially the most fun anyone can have. There is no video game, extreme sport, puzzle, quiz, theatre experience that can compete with those moments you expand the edge of the planet's knowledge envelope. "You get these moments of thrill. There you are, at 3:00 in the morning, and you know something about how we evolved that nobody else in the world knows. It's a thrill of discovery. You make this breakthrough, and you find something. It's this wonderful, wonderful scavenger hunt when you got to the end. It's just so great to

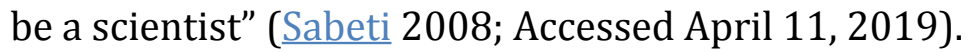

It is a privilege to be paid to spend your time in this pursuit. The privilege may not come with the type of salary/lifestyle society offers other occupations, but it does come with the freedom and the time to explore your own interests in nature/culture and the universe. This may be the best reason to keep the academy away from the logic of the marketplace, where freedom and time belong to others, where finite games fill your days and take you away from the very serious task of playing with nature.

\section{Looking for the next patent, weapon design, or mass-consumable gadget or drug might make you rich, but it's not science.}

Using science resources and funding for science to accomplish these things, and their like, fits extremely well into the neoliberal logic of the marketplace. The incentives and rewards are nicely lined up. These finite games have obvious winners, and lots of losers too. Here is where the Matthew effect (Merton 1988) translates into cash rewards. Nearly all the current incentives for/in the academy have perverse consequences, including patents (you can look ahead to Against Patents in the Academy). Marketplace counter-norms have already won, so it seems. Your "Research Excellence Framework" score matters a lot more than the actual new knowledge you and your colleagues have assembled.

This is why open science looks to build internal economies with its own logic, norms, principles, and rewards. There are lots of ways to be poor and have lots of money; "Money is just one of the many currencies you need to thrive and be happy in a world that requires and rewards attention, reputation, networks, learning, creativity, and tenacity....Progress in 
most academic disciplines now seems to move at the speed of 'instantaneous,' with discoveries building atop one another at a dizzying pace" (Ito and Howe 2016).

The coming logic of abundance for science arrives sooner when you manage your own expectations. Abundance starts are the edge of "enough." Having "few needs, easily met" lets you locate a range of opportunities you might have overlooked. Here you might want to remember that open science is not just about publication access, it is about refactoring the academy to eliminate the sources for bad science, to accelerate the sharing of science objects across the planet, and to reboot the cultural DNA of academic organizations around the logic of abundance (See: Abundance).

People will ask you, "how do you incentivize scientists to do the right thing [...when the wrong thing pays off so well]?" You might respond by saying something like: "how about giving scientists the means to do exceptional work, to have this work shared across the planet, to gather instant feedback from peers around the world, to live simply with plenty of time to do research without racing for funding, to have security of income and access to research tools." Time to do what you are passionate about is a great luxury, and has been for centuries. Setting your own goals, choosing yourself as the person who can contribute and accomplish great work, mentoring others to secure the future of science: these are incentives you can own.

\section{Being a scientist is...}

"Feynman always said that he did physics not for the glory or for awards and prizes but for the fun of it, for the sheer pleasure of finding out how the world works, what makes it tick" (Feynman 2005).

At this point you might be thinking that the science described in this framework is not what you wake up and do every day. Your life may be dominated by demands from your organization for high productivity scores, funded research proposals, and publications in high impact journals; editors nudging you for your peer reviews; assistant vice chancellors pestering you with patent forms to fill out; constant rejections (curse you reviewer three!) and revisions in your own output; courses to teach, lectures to prepare, and grades to give; and, right... home life. All this talk about joy and fun may seem oblique to your actual life.

Have hope. The high-pressure, low-fun career for scientists is not what science needs, and not how it was (and perhaps will not be again soon) designed to operate. We will discover below that infinite science play removes any attachment to glory as a goal. The pursuit of glory is a finite game activity, where a few get this and use it silence the many others who don't. Some decades ago, science was considered a pursuit best done outside of marketplace incentives: 
"[Vannevar] Bush convened a panel of leading academics to formulate a vision for postwar science policy. In July 1945, the panel produced a 192-page document dramatically titled Science: The Endless Frontier. Heralding basic science as the 'seed corn' for all future technological advancement, the report laid out a blueprint for an unprecedented union between government and academia-a national policy aimed at fostering open-ended blue-sky research on a massive scale. Though he was a conservative, Bush laid a groundwork for what Linda Marsa aptly termed a 'New Deal for science,' seeking to preserve a realm where university research was performed free of market dictates.

'It is chiefly in these [academic] institutions that scientists may work in an atmosphere which is relatively free from adverse pressure of convention, prejudice, or commercial necessity,' wrote Bush in Endless Frontier, 'Industry is generally inhibited by preconceived goals, by its own clearly defined standards, and by the constant pressure of commercial necessity.' Of course there are exceptions, he acknowledged, "but even in such cases it is rarely possible to match the universities in respect to the freedom which is so important to scientific discovery"' (Washburn 2008).

This freedom is what you've lost; what open science is determined to regain. You can find a lot of discussions around "academic freedom." Being a scientist carries a great responsibility to maintain a specific variety of this. Again, here's Feynman:

"It is our responsibility as scientists, knowing...the great progress that is the fruit of freedom of thought, to proclaim the value of this freedom, to teach how doubt is not to be feared but welcomed and discussed, and to demand this freedom as our duty to all coming generations" (Feynman 2005).

This "freedom of thought" extends to ideas shared freely within the academic community as gifts from scientists to the entire community. Hyde notes that this "gift" logic runs counter to the logic of the marketplace:

"A gift community puts certain constraints on its members, yes, but these constraints assure the freedom of the gift. 'Academic freedom,' as the term is used in the debate over commercial science, refers to the freedom of ideas, not to the freedom of individuals. Or perhaps we should say that it refers to the freedom of individuals to have their ideas treated as gifts contributed to the group mind and therefore the freedom to participate in that mind" (Hyde 2009).

Being a scientist means giving what you learn, the best you have, to your peers in a sharing community, with the expectation that they will do the same. It is beneficial to remember that when your mother or grandfather was doing science, the academy's position as external to the marketplace was valorized and celebrated. Being a scientist means you can 
demand the freedom, the time, and the resources to investigate your own infinite play: the object of your own study and your singular passion and potential joy.

"There can be occasions when we suddenly and involuntarily find ourselves loving the natural world with a startling intensity, in a burst of emotion which we may not fully understand, and the only word that seems to me to be appropriate for this feeling is joy" (McCarthy 2015; see also Brain Pickings 2018).

\section{Doing science is...}

Science is the most difficult, most ambitious, most challenging pursuit that the human species has ever attempted. Every unknown is integrally linked to the entire infinite playing that is the universe in which we swim. So your unknown-that bit of the game you have chosen to interrogate-is just as important as the next bit. Tackling your unknown is difficult by default (if it wasn't this would already be a "known"). What is really painful is not being in constant, constructive contact with the five, or twelve, or a hundred other scientists somewhere on the planet who are, at this moment, running the exact same thoughts through their minds as you hold in yours.

Open science means you no longer need to consider these colleagues as your "competition." A goal of open science is to connect you with these, your disciplinary siblings, and help you work faster, work better, and have more fun discovering more by working together than you can on your own. These are the people who can help you the most, and who need your expertise the most. Together you can make science stand up and dance through infinite play.

Doing open science means getting to dive into infinite play. Doing science means unleashing your passion for knowledge exploration and diving into your research. Doing science means sparking the same passion for learning in your students. The role of open science in your life and for your research and teaching-and through the places where you work and collaborate-is to release you from manufactured scarcity, ersatz excellence, and the quest for cumulative advantage; from all of the finite games that others use to manage your life for their goals. 


\section{A guide for scientists to become culture change agents for open science}

"I believe that by focussing our attention on communites [sic], groups, clubs and the incentives that they work within we will make more progress, because at some level the incentives for the group are the culture" (Neylon 2015; Accessed June 25, 2019).

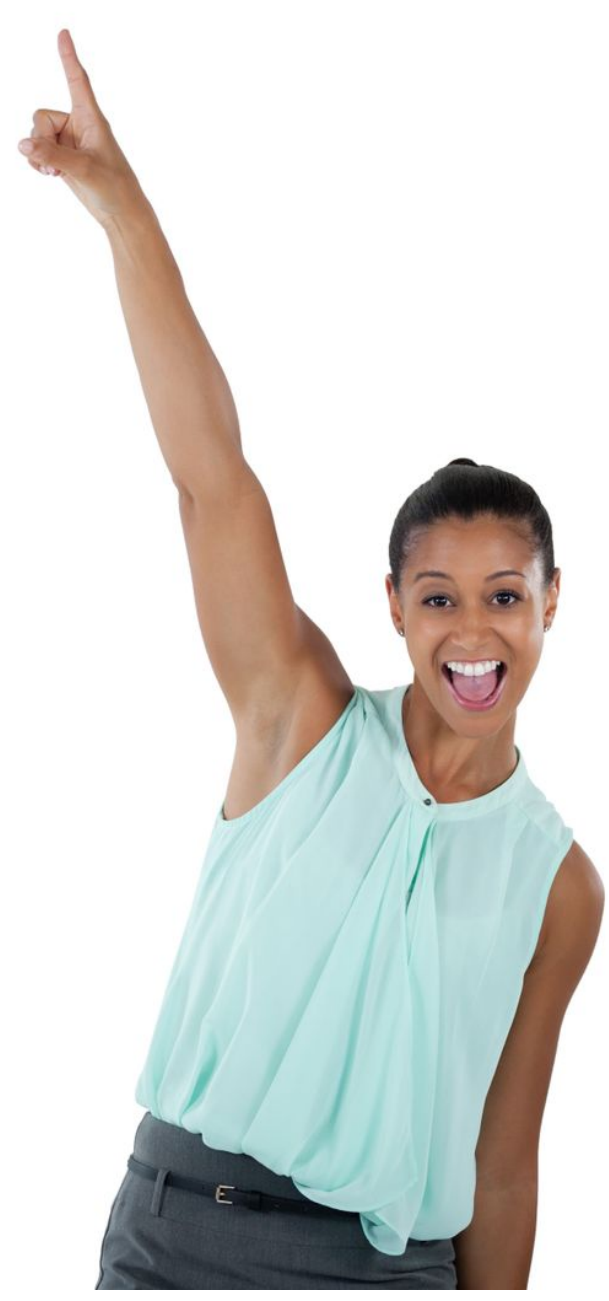

\section{Open science needs you to become a culture change agent.}

Almost everybody you can talk with might agree that changing culture is hard to do. Even getting your arms around the word "culture" is difficult, as we will see later in this chapter. What is becoming more obvious, in this, the opening innings of the $21^{\text {st }}$ century, is that stopping culture from changing is even harder in our daily lives. Outside our jobs, we swim in world-wide jet streams of cultural ideas, and culture-informed gadgets and all the stuff we now buy at Amazon (there's a cultural shift for you, too). Older cultural orders, such as religions and nation-states, struggle to stay current with their traditional messages.

At the same time, adopting to a new technology is often seen as trivial. We live in a stream of continuous small and large technology changes, from the internet, cell phones, and cloud services, to machine learning and artificial intelligence. We integrate these new technologies into our lives and our work as a normal course of how we operate, personally and professionally. 
We spend time in new ways, connecting on social networks, joining teleconferences, editing shared documents and code, sharing ideas, photos, videos, emotional moments, personal triumphs and dead pets. How hard is it to sign up for a new platform and start sharing stories, car rides, or spare bedrooms? Not very hard at all. Today. Today, much of what we do and use was impossible until the moment it became inevitable, like Wikipedia.

Here's the secret: every single one of these technologies that we've integrated into our lives has also changed our culture: the shared practices we use to learn and communicate the knowhow (and the know-where, and know-when) that technology requires to be used. A lot of culture change is just this easy. At the same time, it's really important to note that a great part of these changes involve commercial technology platforms teaching us to become better users for their purposes; optimizing the world around their needs, instead of ours, to achieve an optimal state for their growth (See: Lanier 2014). In this case it's not so much that we are changing culture, but rather some new variant of techno-culture is changing us.

Perhaps this is one reason some open-science promoters are hoping that all the new openscience technology platforms can simply remake us into "open scientists," or that, when we are presented with a list of the benefits that open sharing opportunities create for the academy, we will rationally choose to hop on the open-science wagon. As if that's all we need to do. 


\section{You can make your workflow more open by ...}

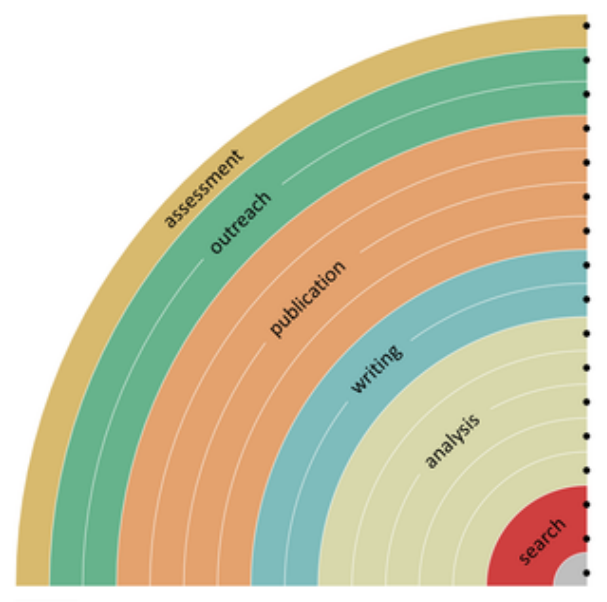

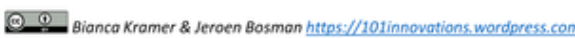

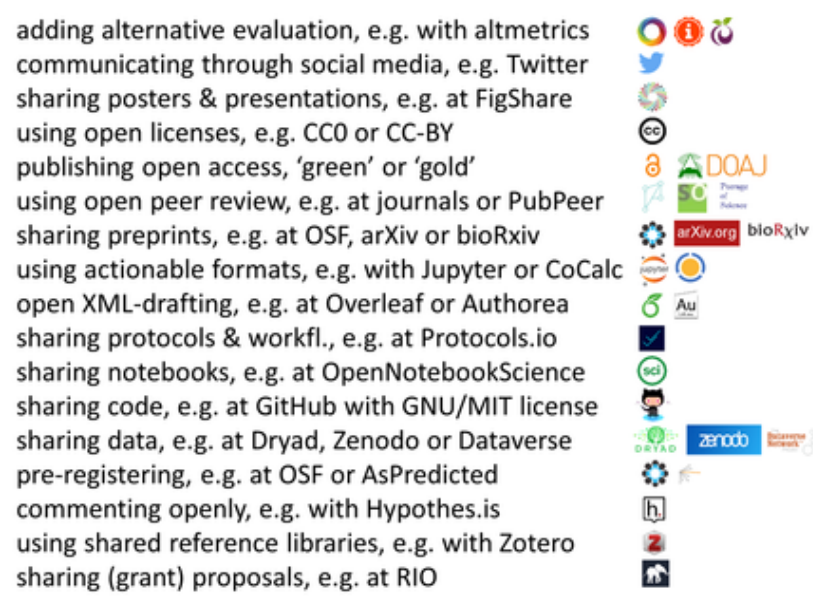

DOl: $10.5281 / z e n o d o .1147025$

Infographic on how to make your workflow more "open." Note: this infographic is years old, and does not represent the current state of thinking about "open." It demonstrates how platforms were/are being set up as solutions instead of tools. DOI: 10.5281/zenodo.1147025

“The culture of today's research universities is built from elements of past universities, including the medieval roles and responsibilities of faculty, the 1819 th century structure of departments, and the mid-20th century research funding model." (Katz et al. 2018)

Universities already carry around a lot of historical cultural baggage, and are said to carry a profound sentiment for resisting change. "Traditions" that uphold hierarchies and concentrate power and decisions at the top are difficult to challenge from below. These need to be challenged in any case. Hundreds of open-research projects happening today across the globe are sharpening the attack.

Actually, many of the "traditions" in play today are not actually old, but rather, they reflect compromises cobbled together in prior decades to corral the growth of universities within layers of neoliberal management. "...[S] ince the eighties, Ginsberg [2011] argues, university administrators have effectively staged a coup. They wrested control of the university from the faculty and oriented the institution itself toward entirely different purposes. It is now commonplace for major universities to put out 'strategic vision documents' that barely mention scholarship or teaching but go on at length about 'the student 
experience,' 'research excellence' (getting grants), collaboration with business or government, and so forth" (Graeber 2019).

This means, of course, that these neoliberal "traditions" mainly insinuated themselves into university cultures since World War II, which suggests they can be similarly removed through intentional cultural change tactics: through recalling and reinstating prior practices (older traditions of scientific norms and sociability-a kind of "retraditionalization" that might also be attractive to conservative culture-change skeptics); and/or replaced with practices that embrace new technologies and social justice. The point is that many of the most fiercely held traditions in the academy are not old, and are the outcomes of recent cultural changes; they are likewise open to change. In fact they are ripe for change.

"Currently, the vast majority of the science pie rewards the building of empiresthat is, the model that has scientists clambering over one another to reach the top" (Steeves 2018, Springer Nature Careers, January 18).

\section{Real change starts with intention}

If technology really can change our culture for its use, then using these platforms and services can help by supporting new cultural behaviors. But the real change we need to focus on is changing the intention that we, and our colleagues bring to our research. It is intent that can make an action kind or unkind; intent that colors how and why we share our research; and intent that is capable of moving the academy away from the perverse incentives that now toxify its culture. Technology alone cannot be expected to provide the cultural anchor for new, open-science behaviors. 


\section{The Work of Culture in Your Open Science Organization}

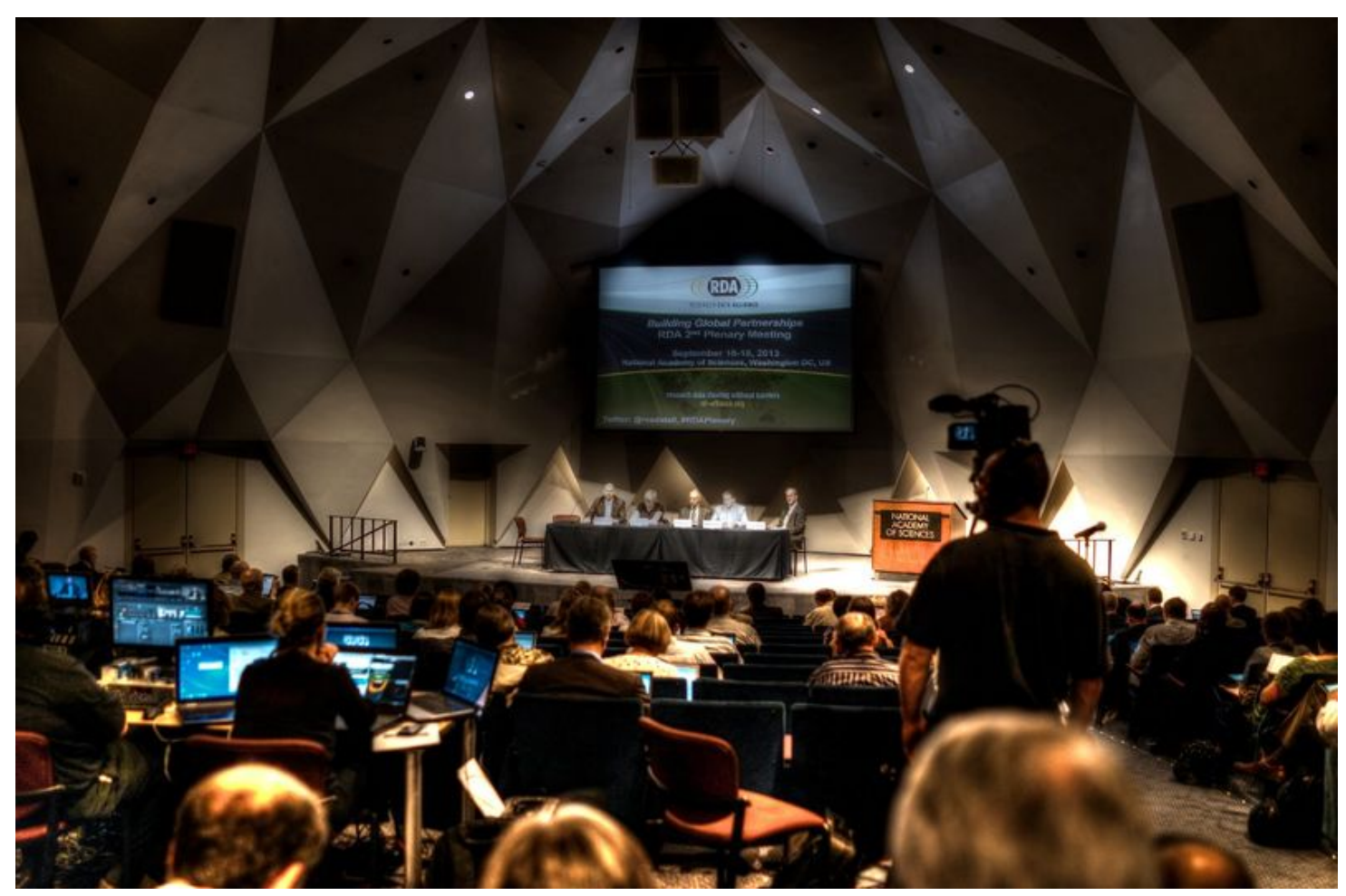

"Religion is a culture of faith; science is a culture of doubt" Richard Feynman (unsourced).

“Don't think of culture as other than accumulated learning that sits inside you as one of your layers of consciousness" (Edwin Schein 2016; Accessed April 4, 2019).

“'Culture' is everything we don't have to do" (Brian Eno 1996; W Magazine)

\section{All the culture that fits: exploring the work of culture to prepare to change it}

We want culture in the academy to work for us, instead of against us. The many meanings of the word "culture" - each with certain claims to capture essential aspects of this spectrum of human proclivity and activity - make the task of outlining a notion of the "work of culture" also a chore of definitions. What is it about culture that can be said to do work? And what work is important for open science? 
One goal of this book is to help scholars who have little or no background in the academic study of culture to gain a sufficient purchase on this notion to become confident, productive agents of culture change for their home institutions, their professional associations and research organizations, and for the academy as a global science endeavor. Like quantum mechanics and machine intelligence, the serious study of culture is not one of these "dip your toes in the shallow end" kind of endeavor. However, with a roadmap through just enough of this contested space, even tenured chemistry professors (or pick your discipline) can become bonafide organizational culture-change agents.

\section{Getting back to basics}

Beginning anthropology classes might spend a month covering the "history of the anthropological ideas of culture." These notions developed first through colonial excursions, and then with missionaries and colonial settlers, and finally ethnographers. A recent (2017) online book for teaching anthropology in community colleges has distilled culture down to a few pages, entitled "The Culture Concept"; Accessed April 4, 2019.) Courses on "organizational culture" are now required in MBA curricula and iSchools.

Arjo Klamer (2017), a Dutch economist, introduces culture to his economics class by adding two meaning domains for this word: culture as the accomplishments of a society (e.g., baroque style as a form of European culture), and culture as creative activity within sectors of the economy (the arts, architecture, music, etc.). His first meaning gives us the adjective "cultured," applied to individuals who exemplify a certain noticeable style; while his second is where you go to when you click on the "culture" link in an online magazine or newspaper.

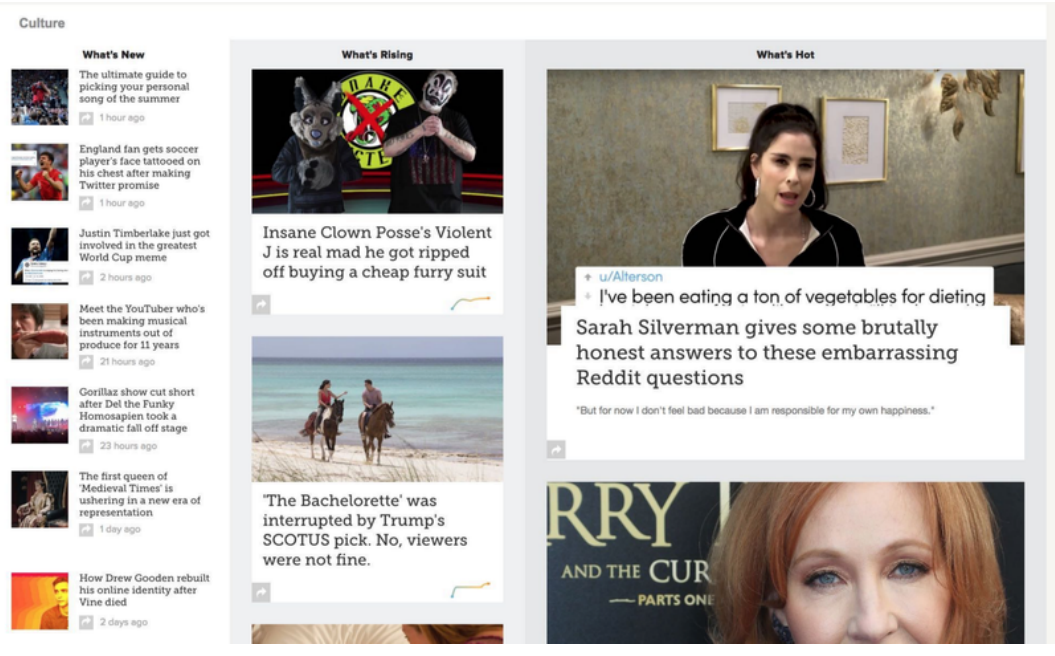

"Culture" is a section in your newspaper/magazine/webzine 


\section{Culture as a process}

Folks who want to use culture and culture change as a resource or a tool to change social groups describe culture as a process. They then offer a method to intercept and guide this process (Marcus and Conner 2014). Organizational management researchers are full of advice on the culture of organizations, but usually fail to look at how this type of culture fits into the larger sense of culture's role in society or in individual identity. Anthropologists describe cultures and how these change without intervention, but little advice on how to intentionally change this. Here, you will find both anthropological and organizational perspectives, just so you are fully comfortable that you've travelled the entire landscape of the term "culture."

\section{Do you own your culture, or does your culture own you?}

"Culture is public because meaning is" (Geertz 1973).

Much of the disputed territory for culture, whether as an object of study, or as a field for intentional change, is centered on how culture is carried more or less unconsciously by the individual. Sometimes it feels as though we've been "marinated" in cultural practices our entire lives: language, cuisine, music, art, and now online content. There is a part of culture that is tacit, embodied, unspoken, and non-conscious. Culture theories tell us this, and they are not wrong. This aspect of culture is often used to demonstrate how difficult it is to manage culture.

\section{A vague, squishy word, indeed}

Jean-Louis Gassée (not an anthropologist; but rather of Apple, BeOS, and Palm fame), in a blog about Intel's "toxic culture" writes:

"Our powerful human emotions are bundled into something we call Culture, itself a vague, squishy word......Culture develops within us in a manner similar to our taste buds: Our gustatory education starts with Mother's milk and accumulates over time. The trouble with our acquired tastes, particularly in the realm of ideas, is that they drop below our consciousness: Raw data are filtered, judged, and labeled before being passed to our conscious, 'rational' processes."

Gassée is pointing out that parts of the repertoire of shared meanings, behaviors, and sentiments that people would label "cultural" are known without any explicit knowledge of how and when we came to know these; and even less ability to describe them.

Schein (2010) calls this a cultural "layer." This layer is learned from birth at home, and then in school, and then in the workplace, where the same tacit layer proves the hardest part to 
change. When your company/university/agency is running on a tacit culture layer, instead of on a reflexive intentional culture layer, it is most vulnerable to becoming toxic.

\section{Science is a reflexive, interrogative activity}

Fortunately, the main aspects of academy culture we are hoping to change can all be made explicit and available to reflexive rebooting. In fact, open science is not reinventing science as much as clearing away the extraneous cultural underbrush (such as journal impact factors) that has collected in the past half-century or so. Scientists can openly interrogate these practices, and collectively move away from perverse incentives, conflicts of interest, and culturally-supported bad behavior in the academy. The leading advice to Silican Valley CEOs today is to avoid "f*cking up your culture" (See also: Don't F*ck Up Your Culture; Accessed May 17, 2019). The academy might want to listen here.

\section{You cannot really avoid culture if you want change}

A good point is worth saying twice: you may be an open-science pioneer who is eager and intent to bring productive changes to the academy, and yet still be uncomfortable with the notion of culture. You might prefer to offer solutions (e.g., coercive rules enforced by governments and funding organizations, novel technology platforms, and manifestos - so many manifestos) that, you hope, would shape "social behavior" without needing to confront or even consider culture. You look at the term "culture" and see a morass of competing meanings, with tangled and complex implications for the use of the term. How do you defend a program to change culture when you can't get any three people in a room to agree on what culture means?

Scientists are many things. Each of these things have something in common: a desire for precision. The "vague, squishy" term "culture" offers very little precision and a whole load of ambiguity and complexity. As a scientist, you already have your hands full of ambiguity and complexity; you are striving to understand the inherent, emergent complexity of the universe. You rely on instruments that achieve ever-better accuracy and precision to help you extract some level of near-certainty to observe your object of study.

Many scientists are dismayed by the sheer amount of fuzziness surrounding the notion of culture. So the project at hand is to un-fuzzy that corner of culture where the academy can work on intentional changes to promote open science. The rest can remain terra incognito. The fact is, you don't need to be an anthropologist to put culture to work in your organization.

In short: the good news is that the cultural work of open science is centered on those aspects of culture that can be intentionally described, discussed, and refactored-even if some of these might later become routine and get framed as default expectations. It's not a bad 
thing to have your active culture also inform the tacit level of culture, it's actually a goal: norms are cultural behaviors and attitudes that have become tacit culture. A norm is when "we open scientists do things like this," and think: why would we do anything else?

\section{Culture: trimmed down to size for the open scientist}

Here we will trim the semantic tangle of the term "culture" to a more specific notion of culture: to the point where it can serve our understanding of how this works and how this fits into the future of the academy. The word "culture" will still hold all of its diverse and multiplex meanings everywhere else, however, here we'll just agree to use it in one specific way to cut through a lot of the semantic shrubbery it has acquired over the centuries and around the globe.

\section{Learning from anthropology}

We can start by looking at some general attributes of "culture." In his 1993 book, Culture, Chris Jenks notes (following Ralph Parsons):

"...for present purposes three prominent keynotes of the discussion [around culture] may be picked out: first, that culture is transmitted, it constitutes a heritage or a social tradition; secondly, that it is learned, it is not a manifestation, in particular content, of man's genetic constitution; and third, that it is shared. Culture, that is, is on the one hand the product of, on the other hand a determinant of, systems of human social interaction" (Jenks 1993: 59).

Lets put these verbs into the following order: learn (first exposure) $\rightarrow$ share (locally) $\rightarrow$ transmit (across space/time). Repeat as needed. This sounds a lot like education, something the academy already does. For the individual, this process is, or can be, a lifelong activity. What Clifford Geertz reminds us is that these cultural activities are public. Nothing is cultural until it is shared. That means these activities are available to study, and to change, and to be changed through intentional intervention (although somewhat less available when they are only tacit).

One easy way to see what Jenks is proposing here is to substitute "language" for "culture;" after all, language is a good part of any society's cultural repertoire. Saying that language is transmitted is to acknowledge that we don't need to invent our own language anew every generation. Saying language is learned explains that we acquire this through learning as children and then hone this learning throughout our lives. To say that language is shared points to a key concept: we need others to make this work; it's called "conversation". In many ways, language is primarily a type of sharing. Other skills and cultural content exhibit these same features. 
The reverse is also true. If a language is not transmitted over time it "dies". If a person doesn't learn a language, they are left outside the conversations that happen in that language. And when a language ceases to be shared in everyday life (e.g., it becomes a "sacred" language that can only be spoken in certain places/times), other language forms will take over in daily life. Languages change all the time. Remember that. They manifest lifelong, tacit cultural practices, and they still change.

\section{Culture comes in community boxes}

"Community, therefore, is where one learns and continues to practice how to 'be social'. At the risk of substituting one indefinable category for another, we could say it is where one acquires 'culture"' (Cohen 1985).

The usual container for a culture is called "community." As an organization grows and governs its own cultural work, you can say that the group becomes a community. You can dive into "community" elsewhere in the Handbook (See: on community). Notions of community will also be threaded into many of the Handbook chapters.

Meaning, Symbols, and Memes; oh my!

Exactly what is learned, transmitted, and shared as culture is complicated. "Meaning" usually pops up here, together with "symbols" (meaning carriers). In many ways anything that can be learned (anything you can get better at by learning this), and that must be shared in order to make sense as something to do (write a song, choose a new fashion statement, enter a conversation, sports, theatre, etc.) becomes culture when the various meanings of that learned behavior are also shared. You cannot have your own private culture. That said, you can have a very small community with its own distinguished cultural behaviors.

Memes are symbols that have been reimagined as cultural-genetic replicators. The analogy to biology is intentional, and meme theorists also talk of culture change as evolution. Since the 1970s, meme theories have been proposed to explain how certain cultural content packages spread and persist.

“[Richard] Dawkin's way of speaking was not meant to suggest that memes are conscious actors, only that they are entities with interests that can be furthered by natural selection. Their interests are not our interests. 'A meme,' [Daniel] Dennett says, 'is an information packet with attitude'" (Gleick 2011).

The notion of a meme is centered on the idea that humans as social beings are shaped by culture the same way their bodies are shaped by their DNA. If you want to explore memes a bit more, here's a good introduction (by Dennett) and some good counter arguments (by Lanier). Here we will talk about meaning and symbols and culture change, but you are 
certainly free to talk about memes and evolution. You can also look into "cultural science," where evolutionary cultural studies are being done.

\section{Culture is a plural noun}

Not grammatically, of course, but we have seen and continue to see around us how cultural notions, skills, and activities are typically multiple, contested, fragile, and liable to change. Individuals tend to privilege those notions, skills, and activities they have invested time to learn (so nobody wants to be forced to use a different language). However, since culture must be shared to be viable, individuals continually find themselves in conversation with others who have differing cultural inventories. Culture is like a life-long song we only sing once, and none of us has been handed the score for the next chorus. We just keep on singing, in multipart harmony.

Of course, culture is not only a noun. Humans are cultural beings. Humans have culture. Humans do culture. Science is a culture. Universities have organizational cultures. Culture is alive on the internet. There is a lot of culture going on all the time. More recent takes on organizational culture reject this as being just some packet of ideas that gets passed around. Today, more than ever before, culture is viral, active, flowing (Appadurai 1996).

\section{The cultural work of social organizations}

Cultural practices and social organizations are intertwined in time and space. Social organizations are the social "appliances," the furniture, that anchor human groups into more durable cultural contexts, which they support and are, in turn, supported by. These contexts expand our capacity for collective action, including economic and political action. Just as we do not need to-or get to-invent our own language, we don't get to invent most of the social groups we intersect in our lives. But we can change them.

In order to pursue the intrinsic cultural work of the academy, we build communities inside organizations that use governance processes to support sharing knowing. We use can our organizations to manage other, social and economic tasks. If knowing is a dance, then community is the dance floor, and the organization is the dance hall.

\section{The social work of cultural organizations}

While this section is about the work of culture, it is good to remember that culture informs the social work of organizations. Later in this section we will look at values, virtues, freedoms, and principles, and at strategies, norms, and rules. All of these translate cultural work into social settings and systems. In some ways, social systems are like petrified conversations: they take the result of discussions and turn them into procedures, work codes, job descriptions, organizational charts, accounting schemes, etc. The important work 
of culture in any organization is to keep the conversations current. This is how an organization learns, pivots, when needed, and reinvents itself.

In the twenty-five years since Jenks' book, culture has seen a lot of new attention. From the academic discipline of "cultural studies" to the cubicles of Silicon Valley start-up companies, the importance of culture for the everyday life and future prospects of societies and corporations has become a central theme. It's high time for the academy to take a culture turn. You can help.

Now you know enough about the various aspects of culture to start rolling up your pants and wading in. You know that culture is (and must be) learned, shared, and transmitted. Most of culture is really vulnerable to intervention or substitution. Culture describes a broad range of human activities and a layer of meaning that is spread over (or under) social activities and organizations. The meanings of culture are all public. You can find them, interrogate them, and, yes, change them. That's the next topic in the Handbook: The task: culture change. 


\section{Performing Open Science Culture}

"Open Science, perhaps more properly termed Open Scholarship in English, represents a culture change in the way stakeholders in the research, education and knowledge exchange communities create, store, share and deliver the outputs of their activity. For universities and other stakeholders to embrace Open Science principles, policies and practices, there needs to be a culture change in these organisations if this transition is to be successfully negotiated" (LERU 2018. Open Science and its Role in Universities: a Roadmap for Cultural Change. Advice Paper 24, May: Accessed, June 12, 2019).

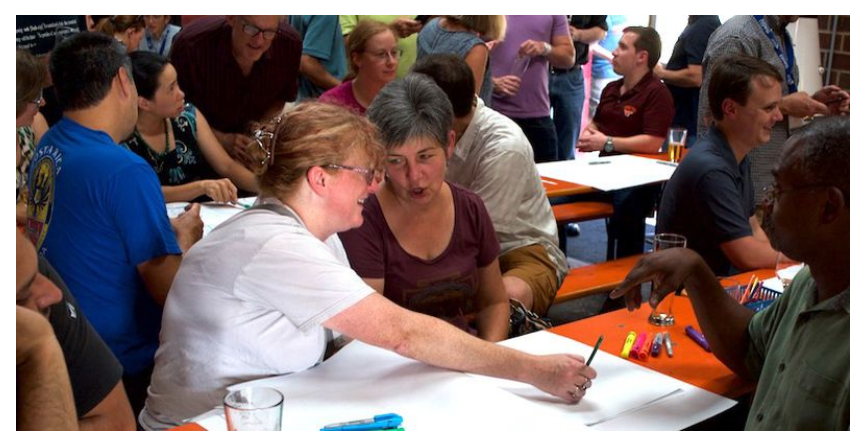

\section{Celebrate open science culture change and values: and then take these personally}

"[Y]ou get what you celebrate in a free culture," Dean Kamen.

"...intelligent failures, as the term implies, must be celebrated so as to encourage more of them" (Edmonson 2019).

"...frustrations can be vented; accomplishments and people spontaneously celebrated. In these moments, more is at play than simple information exchange" (Laloux 2014).

"You need to not only understand your values, but celebrate them..." (Bacon 2009).

In much of the literature on organizational culture, the advice is to "celebrate" values and norms. The term "celebrate" is used as its meaning suggests: first, to acknowledge with a social gathering, and second, to affirm through some shared event. Acknowledgement and affirmation require generosity. In the academy, the term has been mostly used adjectivally as an attribution: "She is a celebrated biologist;" or "Her celebrated work on X explores..." 
Here, however, celebrations are active and verbal. However, balloons and song are not required. Conversations and smiles are just fine. The events can be planned or spontaneous. Large group, or small team. Day long or a momentary. Celebrations are times when you do cultural practices together, with the message that these are really what open scientists $d o$. The important thing here are the three aspects that are happening at the same time in any real celebration.

\section{Celebrations of culture in your workspace: three important aspects}

1. There is a shared social activity.

It might be as simple as reflecting on an intentional culture practice that you are doing together. In the photograph above, participants are designing posters that show innovative projects they hope will acquire some micro-fundings. At the same time they are sharing in the open, transparent funding effort where they will know the results the next day. (See ESIP Funding Friday web page).

Everyone can participate as they wish. Time and resources may be spent to hold these events. Regular and irregular events break the routine of the workplace. Within the event any number of values and accomplishments can be mentioned, or the event can itself celebrate the value of being a community. Alternately, celebrations may be as simple as someone making a positive comment about someone or some activity, and everyone else nodding and smiling. Occasions where time and resources are spent are investments by the organization to its employee community.

2. This social activity requires and rewards a shared positive emotional tone among all who participate.

You do not need to be the most enthusiastic person in the room, but you are expected to actually want to celebrate. Lending your sincere emotional support to the activity is a gift you give to your community. This shared emotional space also opens up the social frame for interpersonal conversations that can build trust, and improve teamwork. Even most introverts can find something to smile about.

3. This activity is meant to be shared within a community.

Going out dancing on your own in a night club after work may be your way of "celebrating life," but here you belong with your team. And that sense of belonging is shared. In fact, these occasions for celebrating are times when "fitting in" ups its game to signal actual belonging. You get to be who you are. The rewards of belonging are gifts from the community to its members. This part of celebrate requires that you've spent some time creating community in your workplace. This usually means that you share building community as a value. Every time you celebrate something/someone you are also celebrating your community. Every sincere smile in the celebration is a gift from the community to its members. 


\section{Celebrations demonstrate generosity}

Celebrating starts with a clear intention: generosity. Even when there is no material sharing, generosity of spirit is present. Lacking this, no event can be called a celebration, even though it may look like one (balloons, songs, whatever). Celebrations are good barometers for the health of your institutional culture. On any one day, you might not be feeling very generous. That is fine, you can still participate with a modicum of generosity. Not feeling generous at all? Something might be wrong with your workplace.

\section{What failed celebrations signal}

You may have had the painful experience of an office party where nobody is feeling generous; where everyone knows that the event is for show only; and where there is no shift in the shared emotional mood that would allow for free conversation. In a culture turned toxic, you can no longer actually celebrate; in fact, genuine generosity will seem out of place.

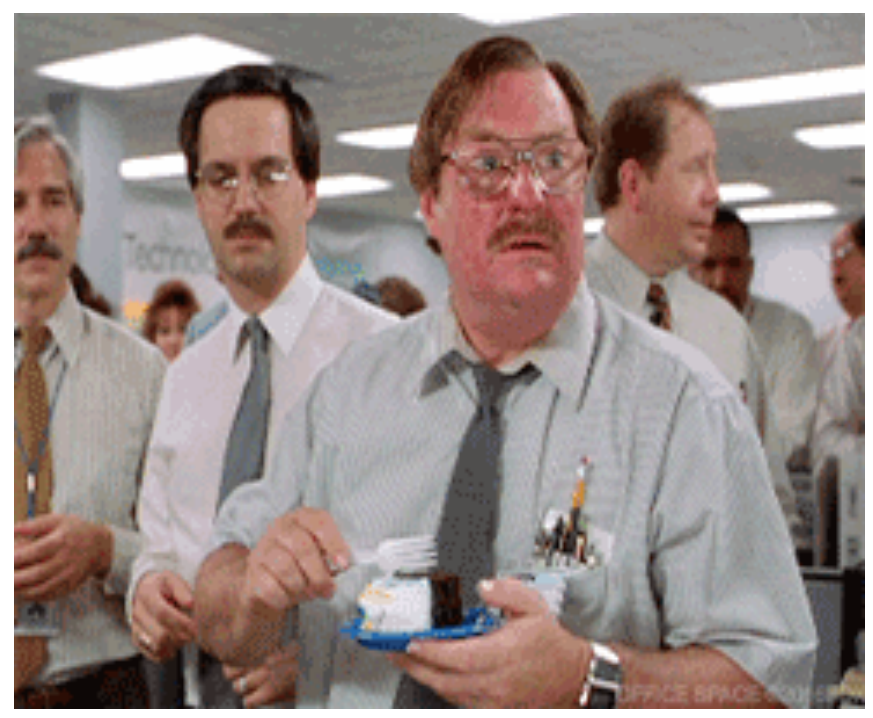

\section{The ratio of people to cake is too big...}

When celebrations fail, it's time to reexamine your culture. This means that celebrating your values (and each other) is also a litmus test on how well your governance is working to help you build a community-another reason to celebrate regularly. Putting up your values on a sign by the front door is not the same as celebrating these. This goes for your academic department or lab as much as it does for a Silicon Valley start-up. 


\section{Celebrate Real Change to bring back the Cynics}

When celebrations succeed, they enliven conversations and diversify the work day. Putting constructive fun into your open science culture change effort is a win for everyone. Academics around you-and, confess, probably you too-have once been or are still somewhat cynical about open science changing the academy. Academics get a lot of mileage from their shared cynicism, it seems. Cynics believe change is impossible. "You'll never incentivize academics to be generous when their career is on the line," they say, and wait for some response they can dismiss.

When you promote incremental changes to academy behaviors, through articulating new norms and pushing for open practices, you demonstrate that change is possible, opening up the door to bigger changes, and allowing cynics to slough off their old beliefs. Shared celebrations of new practices give former cynics an experience of change (however small) and the emotional support to engage in conversations, rather than postured challenges. Some cynics become the most committed change agents when they see change happening around them.

\section{Going Glocal}

This Handbook is designed to be used locally, and to reflect open science notions that are potentially global in scope. There are no precincts on Earth external to science. At the same time, it is just wrong to propose a single set of principles or a pre-imagined vision that locales must follow. Access to scholarly resources over the internet improves their global reach. Local or national infrastructures sustain these resources, and hundreds of academy organizations generate and steward them.

These organizations are imbedded into local education/research cultures that are the actual sites for culture change. Like a giant shoe store, the academy has a broad range of styles and sizes of organizational culture. It holds a vast inventory of different cultural attributes. And yet, to push this analogy is bit, these are still all shoes. The republic of science shares a common knowledge pool, and a collective goal. Your task, as an openscience culture-change agent is to work locally to support this planetary endeavor: science. This means that celebrating the norms of global science also opens up occasions where you can share how this is best articulated in your locale. The smallest locale you have is yourself.

\section{Taking culture change personally}

The task ahead: change the culture in your university; in your professional association; in your laboratory; in your department; in your life. Yes, in your life. The changes you want to make include changes that make your institution more responsive to your needs as a 
scientist, and as a full person showing up to commit your life (some part of it) to the pursuit of scientific knowledge.

Even as individuals can be culture-change agents, cultural practices-particularly shared principles and norms - can, and will change individuals. Acting fiercely egalitarian means becoming fiercely egalitarian. This will have impacts on personal relationship too. Marcus and Conner (2014) call this the "culture cycle."

"To summarize once more how the culture cycle works: Our I's (or selves) both produce and are produced by cultures out-in-the-world, including the customs and artifacts that give shape to our daily interactions, which themselves foster and follow from cultural institutions, which in turn reflect and support our cultures' big ideas', including ideas about what a person is and should be. Because our I's are embedded in cultures, we cannot survive without them. In this way we are like fish in water.

Also like fish in water, we evolved an that we don't notice culture. Indeed...culture is powerful precisely because it is usually invisible to the untrained eye. We are born into culturally saturated worlds, and seldom do we see or discuss how other worlds are arranged. Only when we travel to new places or, say, read a book about cultural psychology do we begin to understand how much culture shapes our selves and appreciate how many different forms cultures can take."

While organizational culture may, and probably should stop short of claiming the "big ideas" of life, there are ideals, norms, and values that are big enough to share within an academy organization; like the norms of great science, and the need for practical wisdom in personal relationships. Much of the change you are looking at is in no way radical, or even novel. These changes may simply be a realignment of the behaviors of those around you (and your own) to how you've already imagined science should operate. In fact, the changes you make might just reduce the feelings of dissonance you have about doing science. Open science is really just asking you to be congruent with the "implicit, collective understandings about how scientific research should be done" (Anderson, et al 2007).

You still have some work to do. To succeed in changing your organizational culture you also need to work on your own person. You will need to develop resistance against those toxic aspects of current academy culture that feed bad science and bad behaviors. To change culture, you need to take science-the whole enchilada of research and teachingpersonally.

"Taking it personally means changing the culture by owning our experiences and holding ourselves and others accountable (Brown 2007)." 
To become a culture-change agent, you will need to celebrate the values of open science in your everyday work activities and in active conversations with others. In the process, you will want to become "authentic" with these values. While you can certainly hold additional values and cultural notions from other aspects of your life (home, religion, politics), you will be much happier when you arrive at a congruence between the open-science values you maintain on the job, and those you hold elsewhere. 


\section{Values, freedoms, and principles of Open Science}

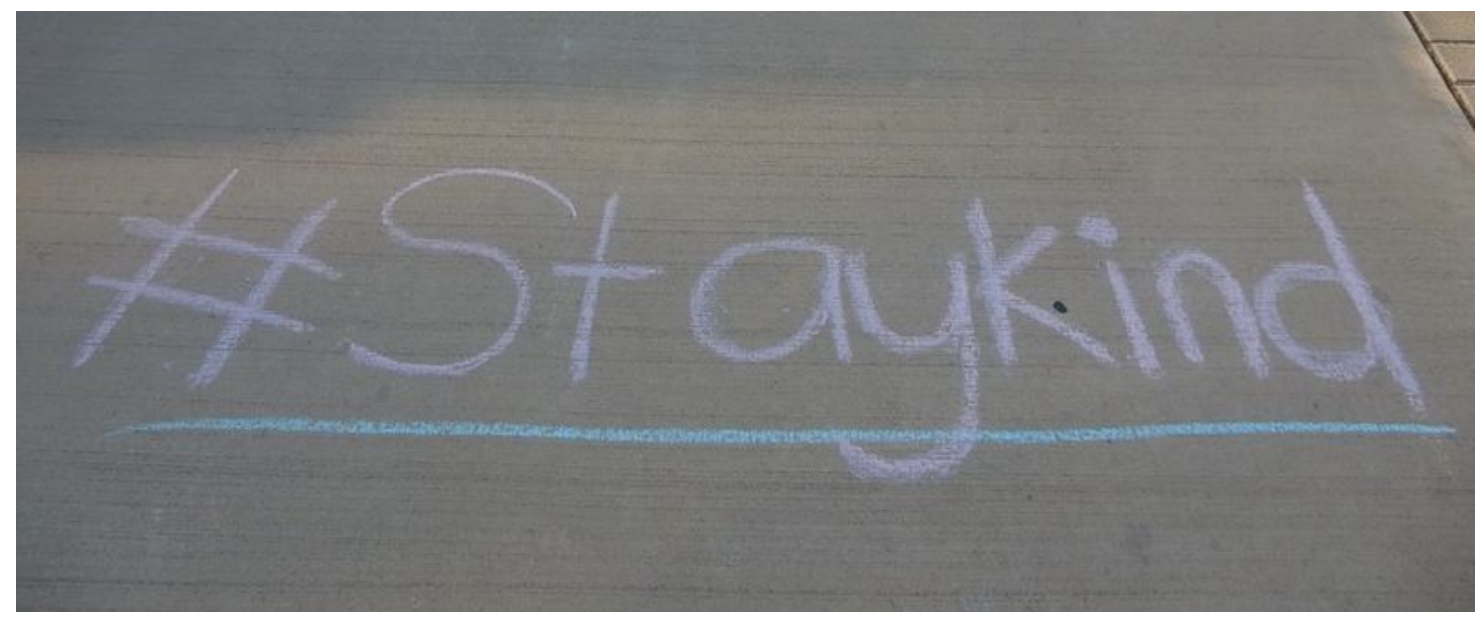

Photo Credit: "\#staykind" by mikecogh is licensed under CC BY-SA 2.0

"Nothing captures our understanding of moral commitment better than the way Marx astutely put it: 'These are my principles; if you don't like them, I've got others,' (That's Groucho Marx, in case you didn't know)" (Benkler 2011).

"Whether you're designing a business model, a website, or a legal statute, values are not an afterthought. Fairness is not something you attend to after the practical decisions about how to improve efficiency or innovation or productivity have been made. Fairness is integral to effective human cooperation. We care about fairness, and when we believe that the systems we inhabit treat us fairly, we are willing to cooperate more effectively" (Benkler, ibid).

"Advocates describe the Open Science ideal simply as science done right, as a public good that should be practiced in connection with society and societal values. Science done right, in this context, includes considerations of social justice and international human rights. The Open Science ideal requires researchers to pay at least as much attention to scientific responsibility as to scientific freedom. Part of that responsibility is to make the scientific literature freely available to all; but Open Science is more than open access. (Holbrook 2019)"

For the open scientist, and for open-science societies and communities, statements of strategies, norms, and rules for open science are expressions of the principles, virtues, and values of open science. Before you can start to talk about open science, you and your 
colleagues need to figure these out. It helps to start with a shared sense of the meanings for these concepts.

\section{Principles, Values, and Virtues... and Freedoms}

Ambiguity warning: again, these words get used in various ways. Here you will find one way to fit this all together. You might prefer other ways, but at least, here is one you can use. Let's unpack these a bit here, starting with values. Klamer (2017) introduces values like this: "Values are qualities of actions, goods, practices, people and social entities that people find good, beneficial, important, useful, beautiful, desirable, constructive and so forth. Values are personal in the sense that individuals experience them as such and they are social in the sense that values derive their impact from being shared among groups of people."

Values can be internal only, or shared. Individuals can value anything they wish, but shared values require cultural work to sustain. Problems arise when there are contradictions between personal and cultural values. The values you hold as an open scientist do not need to be all of your values: you have lots of other values in your life. You might be highly religious, or deeply non-religious, for example. You bring these other values with you, and they help inform the discussion over the values you choose to share in your organization.

Norms are shared values that have become universal inside the culture of your community/group. Norms inform ways of behaving that members perform without much thought, and would feel weird if they didn't do these. Norms are the basis for being able to say, "People like us (open scientists) do things like this." Norms are culturally stronger than rules within teams. When people like us behave like this, you do not need rules to support these behaviors.

Principles (here) are a subset of values that appear to be unquestionable; a kind of supervalue that might also be linked to fundamental meanings and connections to the world. "Scientific principles" are variously described as either the fundamentals of the scientific method, constraints on science (such as falsifiability) or very basic observations of nature (water seeks its own level). In casual use, the term sometimes overlaps with "laws." It is time to get more precise about this term.

"Fairness" is a principle that is often articulated though values such as "equity." The "open" part of "open science" is a value that is also a principle. Other values add facets of meaning to the principle of "openness." "Open" also unpacks to contain other values: findability, accessibility, sharability, etc.. Building a list of values often reveals common principles that they share. Being "principled" (as a person or a community) means that you are true to your principles/values. There is a lot of semantic overlap between "principles" and "norms." 
Norms describe the behaviors (including attitudes) that are informed by shared principles/ values.

The Open Science MOOC has a whole module on open-science principles, as these have been articulated by several organizations. You can use these examples to create your own list of values/principles. But do create your own; then own these and celebrate them. In this work we point to two principles that serve to distinguish open science to non-open science: fierce equality (Fierce Equality) and demand sharing (Demand Sharing). When these become norms, they might just be called "equality" and "sharing".

\section{Virtues to science by}

"Prudence is a virtue, as is temperance, courage and justice. These are the socalled cardinal virtues that we find in the Nichomachean Ethics of Aristotle. Together with the theological virtues faith, hope and love, they constitute the seven classical virtues" (Klamer 2017).

"Management is doing things right; leadership is doing the right things" (Drucker 2001).

Virtues are values that have ethical meaning for you. These are not simply good to hold/do because they make sense; they are good to hold/do because they are the right thing. Virtues are not limited to just those found in books. You can articulate your own.

You can make a virtue from any value you hold as an ethical position. For example, dietary value choices might be virtues. For example: "I would never eat meat" expresses a virtue assuming you consider this an ethical decision. In contrast, other dietary choices might be aesthetic values ("I only drink single malt whisky"); or they can have a medical reason ("I'm allergic to peanuts"). These are not potential virtues.

A virtue that needs a lot of work in the academy is kindness (See: Kindness). The idea that kindness might not be essential for the academy should be seen as bizarre. All learning happens through the kindness of shared knowing. The lack of kindness as a virtue has been linked to idealized hyper-masculinity (and the associated lack of ability/inclination to do emotional labor) (Schultz 2003) and hyper-competitiveness. Both of these are toxic for the academy. if your organization is ignoring or violating its virtues, you have a real problem. Shared virtues, like other shared values, can, over time become norms in the culture of a community. People like us open scientists hold these virtues.

\section{Open Science Freedoms}

"It is our responsibility as scientists, knowing the great progress and great value of a satisfactory philosophy of ignorance, the great progress that is the fruit of freedom of thought, to proclaim the value of this freedom, to teach how doubt is 
not to be feared but welcomed and discussed, and to demand this freedom as our duty to all coming generations" (Feynman et al 2005)

"Academic freedom" is larger, older, and more fundamental as a principle than the movement to open science. This freedom has also been abused in places (such as autocratic governments) and for purposes (neoliberal logics) that obstruct the academy's defense of this, its primary principle. The fundamental nature of academic freedom was written into the Magna Charta Universitatum on the 900th anniversary of the founding of Bologna University, and signed by more than 700 universities across the globe.

Open science is another weapon in the defense of academic freedom. The pursuit of demand sharing promotes the free flow of research objects across nations; the shepherding of any/all research within sustainable repositories; and the demand for state support to maintain and improve these resources. The pursuit of fierce equality promotes wide access to academy resources, and inclusion of research findings from all persons.

\section{What are the freedoms that open science brings to the academy?}

Along with its values and principles, its standards and norms, open science may also include certain new freedoms similar to those presented by the open-source software movement. (See: The Free Software Definition <https://www.gnu.org/philosophy/freesw.html>; Accessed May 15, 2019).

This brings up the question: is open science also "free science" (free as in "speech" not as in "beer")? Since the scope of open science is available for debate and to local formations, there is no universal answer to this question, but there are some ideas that might inform these formations.

One leg of open science is "open access" to research objects. Peter Suber; Accessed May 15, 2019; see also Suber 2012) offers an excellent overview of this topic. He notes that the current push for open access does not require "universal access" in this, its initial moment. Today, open access offers an alternative to paywalled subscription access to academy resources. When you discuss open science with others at work, you will need to decide the scope of open access your organization would like to promote. So let's explore this scope a bit. You will have your own conversations over freedoms as these are implied and supported by open science (or libra science).

\section{Possible freedoms your open science endeavor can consider}

- The freedom to access academy resources from anywhere. We do have the internet.

- The freedom to interrogate the methods/data/software of any research result in the system. Access is a precondition of this. 
- The freedom to reuse academy resources.

- The freedom to add to the academy's corpus of research objects; subject to the rules of the repository applicable to all (e.g., provision of data).

- The freedom to copy, mine, and analyze collections of research objects.

- The freedom to be kind to one another in all actives of the academy.

- The freedom to request help and receive kindness.

- The freedom to participate equally in conversations, discussions, and online forums.

- The freedom to always choose to do the right thing now, and not delay moral action.

- The freedom to point out infractions of community rules and principles without retaliation.

- The freedom to express the joy of doing science play.

Add your own freedoms to this list. And remember that freedoms are worth fighting for, as Toni Morrison reminds us.

"There may come a time when we-students, faculty, administrators, artists, and parents-will have to fight hard for education, fight hard for uncorrupted science (not the ideological or racist science); for sound social history, apolitical anthropology (not strategies of control); for the integrity of art (not its celebrity). There may indeed come a time when universities may have to fight for the privilege of intellectual freedom" (Morrison 2019).

\section{Use your freedoms to point toward justice}

Discussions of open science as a movement toward social justice practices within science, and societal justice outcomes as a concern for science should be a prominent aspect, and an active conversation, of how you arrive at fierce equality in your open-science organization. Grahe et al (2020) is a good starting place to pick up the concepts of justice in/through the academy.

\section{New behaviors will lead to new attitudes: build action into your culture change process}

In building or changing the culture of your organization, the first, and an ongoing, task for you and your organization is to discuss and agree upon the values you want to share. The process of culture change in your organization begins with a discussion about values, then 
it builds statements that support these built as strategies, norms, and rules. Then it looks at how things get done, at the practices that apply to getting to decisions and doing work, and realigns these behaviors with its shared value statements. After that, members of the organization continue to refactor how things get discussed, decided, and done, molding processes and behaviors to satisfy not just the boundaries of these values, but to express and defend their core principles. If you skipped The Work of Culture (above), you might want to take a look. Over time, these behaviors become shared norms. People like us open scientists here would not think of doing anything else. The whole process of how to do this is described below. 


\section{Kindness, Culture, and Caring: The Open Science Way}

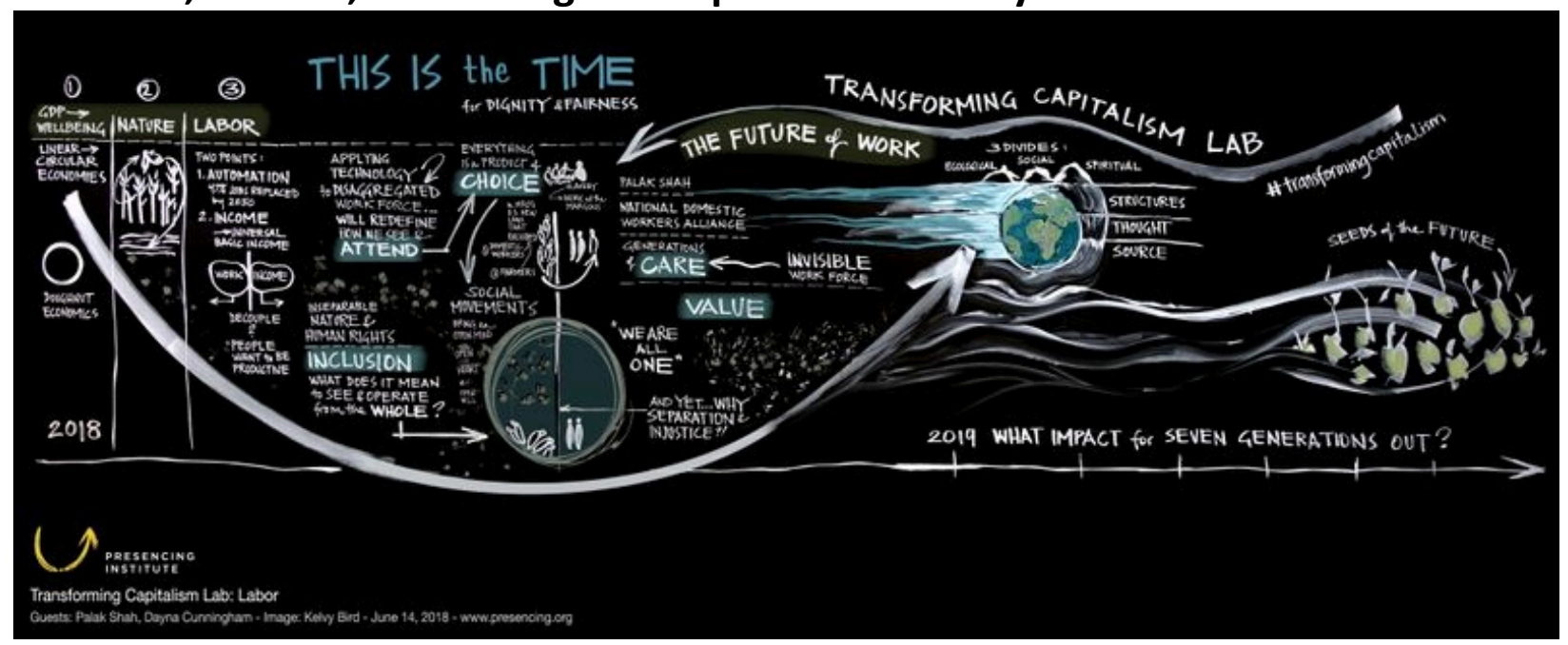

"So it's kind of like if your house catches on fire. The bad news is there is no fire brigade. The good news is random people apparate from nowhere, put out the fire and leave without expecting payment or praise. ...I was trying to think of the right model to describe this form of random acts of kindness by geeky strangers. ...You know, it's just like the hail goes out and people are ready to help. And it turns out this model is everywhere, once you start looking for it" Jonathan Zittrain, Ted Talk 2009.

\section{Becoming an Open Scientist}

There are lots of ways that the rational, logical, hyper-competitive, winner-take-all, zerosum, prisoner's dilemma, nice-guys-finish-last, single-bottom-line, annual-productivity ratchet - or add your adjective here-mindset is just wrong for sustaining the academy and bad for science. For decades now, the same neo-liberal economic schemes that have been used to reshape how governments budget their funds have also made dramatic and disturbing inroads into university budgets and governance. Open science can show how that trend is a race to the bottom for universities. What do you say, we turn around and go another way?

"I have learnt silence from the talkative, tolerance from the intolerant and kindness from the unkind." Khalil Ghibran, Sand and Foam. 


\section{A century without kindness: the impact of external logics}

The banishment of kindness as a necessary part of being an academic,- - just one more feature of adopting the neoliberal marketplace logic, and another effect of hypermasculinity in the workplace-allows academics to defer judgements about kindness:

"We want to argue, however, that although kindness is a commonplace in pedagogical encounters, easily recognisable by its presence or absence, attending to it can be subversive of neo-liberal assumptions that place value on utility and cost above other human values" (Clegg and Rowland 2010).

The word for kindness in Latin is humanitas: kindness makes us human. "[T]he Roman Emperor Marcus Aurelius, a leading Stoic philosopher, speaks of kindness as 'mankind's [sic] greatest delight' (Philips and Taylor 2009,18). In Aristotle's teachings, kindness is a component of phronesis: an entire type of "practical wisdom" that we've slowly devalued over the past 300 years (Juarrero 1999) [ and you can blame Hume and Kant and all the usual suspects for this]. Phronesis combines virtue with a notion of adult comprehension: a way of knowing the right thing to do in all circumstances. It has little to do with intellection, and everything to do with broad experience and learning.

"Kindness is not deference, not conflict-aversion, not niceness or politeness. It's a quality of grounded, dignified, powerful warmth. It's the acumen that allows you to see other people with exquisite precision, and to know that you love them in detail" (Academics Taking Action 2018) [Lab Notes on Power in Academia <http://sophiatintori.com/zine/readable concatenated.pdf> Accessed October 3, 2019].

The road to a doctorate is long and difficult, and so adding another layer of learning to the process might seem short-sighted. And yet avoiding learning phronesis in your daily life is probably not any easier than practicing this, since the absence of phronesis leads to serial mistakes in moral and practical judgement, any one of which can be "career defining" in a negative sense. "Practical wisdom" is integral to "doing the right thing" while you learn to "do the thing right." Doing the right thing often includes knowing how to exercise kindness with others.

A child can show kindness, and we welcome this. An adult (one who has learned some phronesis) can act in ways that are kinder than a child, because this adult is experienced in a broader range of social circumstances and personal relationships. An adult can be-to use the Yiddish-a mensch. And a mensch can be kinder than a non-mensch or a pre-mensch. 


\section{Kindness starts with intention}

Real kindness begins with a clear intention. This adds an important aspect of selfjudgement to its base. Without this aspect you cannot actually be kind, even if others might interpret what you are doing as being kind. How do you actually judge your intentions, particularly in relationships with other people and things? Something to contemplate. Also note: Clegg and Rowland (2010) remind us that kindness is not equated with leniency or "being nice." Real kindness uses courage to articulate accurate observations and open learning moments that can be difficult and painful for both parties.

\section{Kindness is something you learn and do}

Kindness is a normative human practice in a wide range of social frames: parenting, friendship, governance, teaching, caregiving, civil interactions. Zittrain (above) reminds us that the internet was built on kindness and generosity.

In nearly every human social endeavor, kindness matters. Even in highly-competitive sporting events, "sportsmanship" is highly valued, and is actually an internal normative form of kindness. Why should kindness, and critical interrogations about its role, be absent from research and management in the academy?

Like rationality, kindness is a form of practice, not an emotion. You can no more "feel" kind than you can "feel" rational. Unlike rationality, kindness necessarily involves others: their perspectives and needs. Kindness can and will also be judged by others for its qualities. Is it genuine? Is it motivated by a need to be perceived as kind? Is it effective in performing its intention? What is its intention? In the academy where intellectual judgements run wide and deep, kindness opens up another opportunity to be judged. But so does being unkind. Or it should. For decades, the lack of kindness in our research institutions and workplaces has gone unremarked. It is time to remark these.

\section{Culture provides meaning to intentions}

Again, kindness begins with intention. The same activity with different intentions can be a kind, caring conversation, or it can be a cruel interrogation. Intentions are themselves colored by culture. Culture provides a layer of shared meaning/learning that helps the individual (both the intend-er and the intend-ee) discover and interpret shared meaning as intended. You and the other person can answer the question: what did you mean?

The social world always contains this layer of culture. There is no society without it. Individuals hold this layer as a shared/learned resource. The cultural values you bring to your open science organization can assemble the meanings that add clear intentions to shared kindness. Just as some institutional cultures today-and inside the academysupport bullying and demeaning actions (NAS et al. 2018). 
One feature of kindness is that it enables both halves of the double meaning of the term "care." To really care about someone or something, you need to tap into genuine kindness. To care for someone or something can merely be a job. But this job is also reduced without the impulse of kindness. That is why it is time to...

\section{Put care back in your career}

"[B]y infusing bureaucratic maintenance work with an ethic of care, we can challenge contemporary workplace attitudes surrounding "productivity" and "efficiency," moving toward the recognition of maintenance itself as a valued contribution. We can also broaden access to systems of information, thereby supporting its generative value..." (Maintainers et al. 2019).

The Maintainers < http://themaintainers.org/> extend an ethic of care to each other and to their work: they keep everything running, instead of inventing new stuff. This ethic is born in kindness, and requires a level of humility not casually found in the academy, where intellectual heroics overshadow moral choices.

Nell Noddings, who is a "care theorist," someone who makes "the caring relation basic in moral theory" (2003), looks to recenter care as a normative behavior in education and the academy. She also separates the care that is expected in work (for example, doing something really well, or managing the needs of a student/patient) as a conformity to a workplace ethic, from caring: human acts "done out of love and natural inclination" (Noddings 1988). What really works - in teaching and learning, and in team dynamics for collaborative research-is not completing the task of due-diligence, but rather building a framework of mutual caring nurtured from authentic kindness.

Bringing care into this discussion has now moved us away from communities, cohorts, and institutions. Care directs us back to intentions that are articulated in culture, but which also speak to being human in a mutually responsible human environment: a phrase not usually descriptive of the academy. "[W]e are led to redefine responsibility as response-ability, the ability to respond positively to others and not just to fulfill assigned duties" (ibid).

Open science is also science done through care and kindness: science that much more resembles the model of peer production within a commons, than it does a winner-take-all corporate struggle. "[W]ithout receiving conventional, tangible payments or favors in return, peers exercise kindness, benevolence, charity and generosity" (Benkler and Nissenbaum 2006). Open science demands new levels of response-abilities: based on new and expanded academic freedoms (See: Values, freedoms and principles) and internetenabled collaborations grounded in what Fitzpatrick (2019) calls "generous thinking."

"This is generous thinking: listening to one another, recognizing that we have as much to learn as we do to teach, finding ways to use our collective knowledge for 
the public good. From the broadest rethinking of our political and institutional landscape, to developing new ways of working in public, to sharing our ways of reading, to focusing on the most intimate practice of listening - at each level, we must be connected to, fully part of, the world around us" (Fitzpatrick 2019).

Generous thinking expands here, emerging out from the university to help heal the troubles of the surrounding communities through active caring, and also to grow publics that can continue the work of caring outside the academy and among themselves.

\section{Coda:}

There are a lot more articles and books about the history of kindness and care that point out how these virtues were heralded as the basis of human happiness for centuries, and only recently (last 3-400 years) have these been eclipsed by more individualistic moral models (thanks to Hobbes, Kant, etc. - the usual suspects). So... practicing open science may also be good for your happiness. Doing open science can improve your happiness, and the happiness of those around you. How about that? 


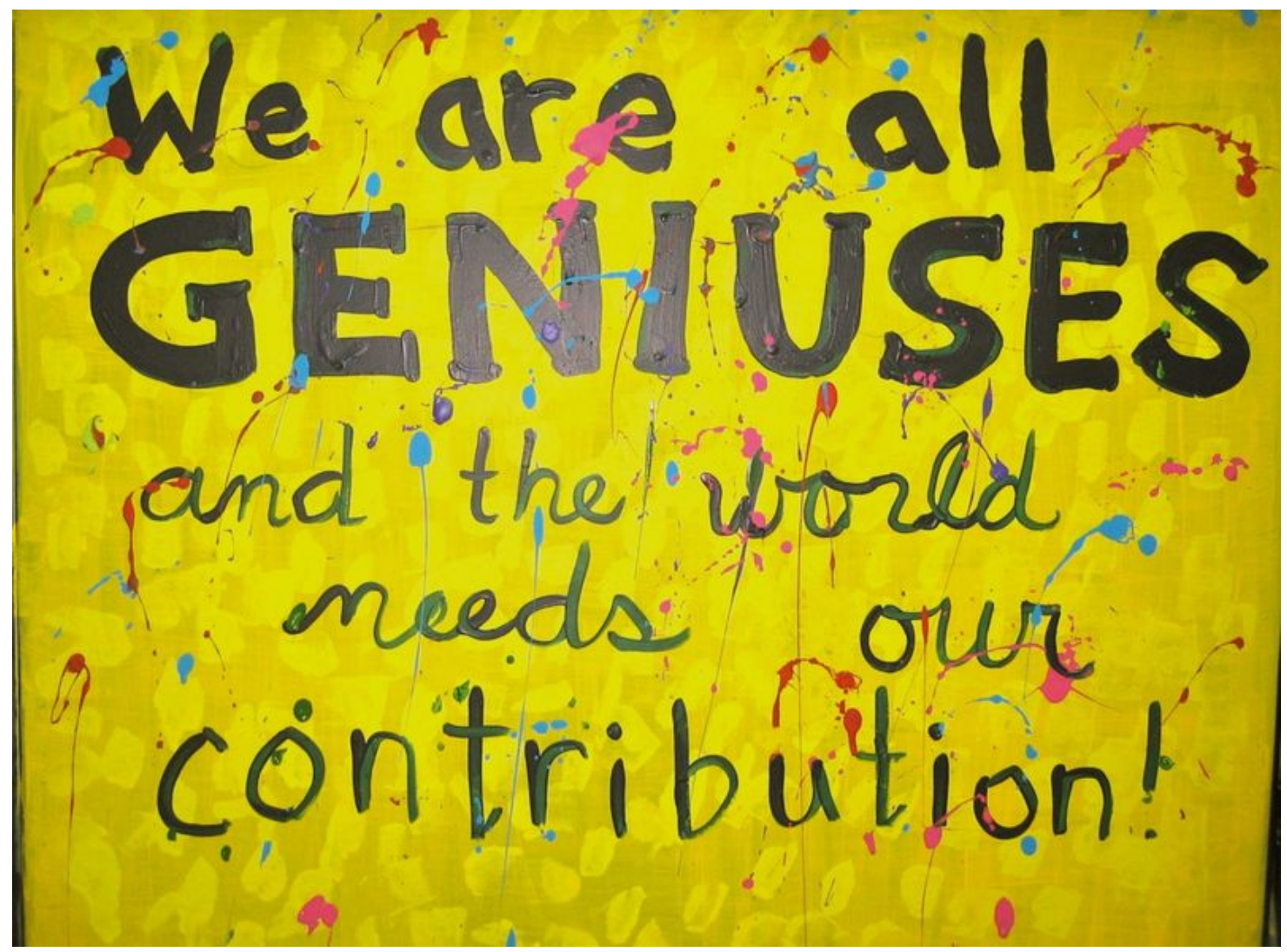

Photo Credit: Denise Krebs on Flickr. CC by 2.0

\section{The Onlyness of the Career Open Scientist}

"Through the power of onlyness, an individual conceives an idea born of his narrative, nurtures it with the help of a community that embraces it, and, through shared action, makes the idea powerful enough to dent the world" (Merchant 2017)

When we look ahead at some near-future, open-science-based academy, we can point to a new science workplace solidly anchored into its own logic, with internal values that reveal its core practices. Each open organization operationalizes these values locally.

Open science is aspirational because science is hopeful: it aspires to add significant new knowledge to human understanding. The open-science movement started with open access to research results. This struggle continues. The current pandemic may accelerate this process. Next, open science needs to move to implement cultural practices that enable Demand Sharing and Fierce Equality at all levels and organizations. Certain common 
notions about academy practices-certain notions inherited from decades of science infested by toxic cultural habits and perverse incentives-will need to be interrogated as open science looks to open cultural practices to shape how it changes in the future. Let's examine some of these practices.

\section{On Onlyness}

But first. The notion of, and the term "onlyness," is from Nilofer Merchant* (2017), an entrepreneur/business consultant (See also: $<$ https://nilofermerchant.com/>; Accessed May 12, 2020). Merchant penned this term to highlight how the quality of ideas in any group springs from the distinct contribution that each person can bring into conversation, when they speak from the totality of their own knowing and being (See: Knowing and conversation). She then outlines how to optimize for this potential. Most teams and organizations downplay, or worse, prohibit, this potential, asking team members to be flexible generalists and leave their individual genius at home. They ask their members/ employees to "fit in" instead inviting them to belong on their own terms.

By locating "diversity" and "inclusion" in the distinct features of each individual, Merchant re-places the standard arguments for these values within teams and organizations. No tokenism permitted here. Instead, we find a keen respect for the distinct biographies of learning and knowing carried by each person in their whole being. As Merchant (2017) notes; "To claim yourself as whole is to assert your own value-not because everything about you is perfect but because it is all perfectly yours. This acceptance of your full self is nonnegotiable in claiming the power of onlyness. If you can't value what you alone have to offer, no one else can either."

Onlyness belongs to every individual to the extent they make a claim about it. It is not exclusive or elite; not just a property of egoistic narcissists. It does not automatically lead to assholish behaviors. It is not acquired from membership in a population cohort or a generation, even though it is socially attuned. It is informed throughout your biography, and includes what you alone can bring to your family, your society, and the planet. Einstein's housekeeper had as much onlyness as did Einstein, only Einstein managed to explore and mine his to the advantage of his ability for creative insight.

Very much in line with recent theories of organizational knowledge management (See: Demand sharing and the power of pull), onlyness powers innovation and creativity within teams and projects by surfacing insights across vital conversations. This also amplifies the value of networks over hierarchies. Open innovation studies have shown that "marginal individuals" contribute significantly to solving grand challenges online, in part, by reframing the problems: "The ability of marginal individuals, with different perspectives and heuristics, to come up with novel solutions to broadcast problems, indicates that they may be conceiving of a given problem in a different way than the seeker. Thus problems 
should not be considered as fixed and given but open to redefinition by the solvers themselves" (Jeppesen and Lakhani 2010). Each team member has a contribution to make to this conversation. No more deference to the highest paid person in the room. Are you looking for better conversations (of course you are), then help each person in your research network explore and claim their onlyness.

"[T] he new research suggests that our everyday thinking and learning is strikingly continuous with scientific thinking and learning. The pre-schoolers see probabilistic evidence and revise hypotheses, but they don't necessarily know that that is what they are doing_nor indeed do ordinary adults" (Gopnik 2012).

\section{Onlyness in learning}

There is no textbook sufficient to map the individual's journey into science. There is no common, model individual open scientist: no career best practice, no business-school recipe for success can identify which scientist will excel in their research endeavor. There is no "mold" for an open scientist. Like a concert violinist, a scientist must master techniques and become proficient in their practice and precise in their methods. But that's more like saying they've stopped being children and are now adults. Or, in a curious fashion, that they've stopped trying to be so adult, and have opened up their internal child-driven curiosity and wonder. To be an open scientist, as Berger (2014) notes: "We must become, in a word, neotenous (neoteny being a biological term that describes the retention of childlike attributes in adulthood). To do so, we must rediscover the tool that kids use so well in those early years: the question."

Knowing what the best questions are and how these are situated into the landscape of scientific discovery is the admission price to enter the "knowledge club" (Accessed May 18, 2020) of science. The other side of wonder is rigor. Rigor is learned later in school. You cannot have wonder without rigor. The reverse is also true. "[R]igor cannot be sustained without wonder; and without both capacities, creativity - and innovation-will suffer" (Nixon 2020). However, rigor is not what drives the individual capacity for science. It's a launch utility, not a fuel. You can teach methods and you can learn content. That gets you to the door of science. To open it, you need to apply your own brand of onlyness.

"It was opal and this was something I knew, something I could draw a circle around and testify to as being true. While looking at the graph, I thought about how I now knew something for certain that only an hour ago had been an absolute unknown, and I slowly began to appreciate how my life had just changed.

I was the only person in an infinite exploding universe who knew that this powder was made of opal. In a wide, wide world, full of unimaginable numbers of people, I was-in addition to being small and insufficient—special. I was not only a quirky 
bundle of genes, but I was also unique existentially, because of the tiny detail that I knew about Creation, because of what I had seen and then understood" (Jahren 2016).

Onlyness here means deep and broad individuality and intellectual curiosity: open scientists are deep into their own specific passion and love for some aspect of science, and their own corner of the unknown in the infinite play of science. Anyone who has completed a dissertation knows the onlyness - and also the loneliness - of understanding something deeper than their books, better than their advisors, and newer than anyone else. Open scientists are also broad enough in their sense of how science works and in the landscape of methods and literature to see the larger picture of open science practice shared in their discipline and beyond.

Onlyness is the reason why scientists can uncover astonishing new insights. By "scientists" here, we mean all students of science, all members of a science team (data nerds, technical specialists, grad students, and principle investigators), in fact, all people on the planet who find that their curiosity - and their own life-to-right-now - has moved them to a distinct point of understanding: "You're standing in a spot in the world that only you stand in, a function of your history and experiences, visions, and hopes. From this spot where only you stand, you offer a distinct point of view, novel insights, and even groundbreaking ideas. Now that you can grow and realize those ideas through the power of networks, you have a new lever to move the world" (Merchant 2017). Onlyness is why your dissertation nearly drove you insane, since it required you to dive deep into a personal journey of discovery that now means you know more-and you know differently—from anyone else on the planet.

If professional scientists act similarly, this similarity comes from the shared depth of their appreciation for the "role of ignorance and the importance of uncertainty" in science (See: Brain Pickings; Accessed May 5, 2020). Rather like we all are in the current situation (now being May of 2020), scientists are, and have always been, alone, even when together.

\section{Onlyness and fierce equality}

Institutional prestige is a profound drag on the potential for networked science. If your administration has a plan to "win" the college ratings game, this plan will only make doing science harder. It makes being a scientist less rewarding. Playing finite games of chasing arbitrary metrics or bullshit prestige drags scientists away from the infinite play of actually doing science. In a world where science happens elsewhere, the first thing your campus can do is become more attached to all those academy "elsewheres" that can amplify your inhouse efforts.

The best thing your campus can do is to became that really attractive "elsewhere" to which others want to attach themselves. This means opening up to demand sharing. Once science 
gets funded across a broad spectrum of institutions and across the globe, online collaboratives will form, and work together, and create new knowing without regard to game-able institutional rankings. The entire academy will become more nimble, creativity will quicken, and good work will find its rewards outside of current reputation schemes.

"One will weave the canvas; another will fell a tree by the light of his ax. Yet another will forge nails, and there will be others who observe the stars to learn how to navigate. And yet al.l will be as one. Building a boat isn't about weaving canvas, forging nails, or reading the sky. It's about giving a shared taste for the sea, by the light of which you will see nothing contradictory but rather a community of love" (Saint-Exupéry 1948; translation: <https://quoteinvestigator.com/ 2015/08/25/sea/>; Accessed May 11, 2020).

The future of open science will be much more distributed and democratic. Open scientists work wherever their research and teaching acumen is needed and supported. The perverse lure of bullshit-prestige institutions disappears as great work emerges from highly diverse teams in hundreds of institutions and locales across the planet, and along the internet.

Instead of boasting of their employment at some famous university or lab, open scientists and their in-house and online teams are deep into infinite play wherever they are employed. They shape the culture of their teams, bending this toward fierce equality and demand sharing. Their combined onlynesses serves to push the team effort beyond what any one of them might do. They fill open repositories with new data and findings. They care for their work together, and for each other as people. They celebrate their team culture. A great team in a sad organization with a toxic culture works better than a sad team in a great organization.

\section{Onlyness against neoliberal organizational metrics}

The notion that a university can increase managerial control over research practices using performance-based funding schemes, and so to capture year-by-year productivity gains, has been tried in various places on the globe. But the practice of top-down, goal-driven, productivity management translates poorly from the commercial world (where this is also failing) into the academy. Metrics applied in this manner are highly susceptible to Goodhart's law, and subsequent gaming attempts. The best incentives for better science are those goods internal to the professional practices of doing science. The best way to improve on these is to support governance practices that open up more avenues for sharing and knowing.

There is an authentic "meritocracy," here, not the artificial sort claimed by prestigious organizations. A fluid, dynamic, emergent shared sense of where new knowing is being forged. In the interconnected intellectual rooms of online science communities, the 
acceleration of knowing and discovery through access to open shared resources, active, global collaborations, and diverse team-building assembles shared intelligence to solve wicked problems. There is no organizational strategic plan, no business model, no tactical hiring that can match open innovation collaboratives that push the boundaries and change the rules of their infinite playing together. The merit belongs to the team, and to the work. What the scientists get is the joy and wonder of a lifetime of science play.

\section{Open science optimizes resources when onlyness gets support across organizations}

Doing open science gets a boost when the culture of open science is shared and celebrated at the top level of academy institutions - whether its a college, a university, a learned society, or a science agency. Under these circumstances, institutional values and their shared meanings cascade down across all departments, labs, and teams. With solid, toplevel institutional support, teams build their own shared mini-cultures to encourage caring and rigor.

The cultural project of open science is actually quite small. As much as open science is just "science done right" and a "return to former science norms," the professed culture of most science organizations really only needs to be rearticulated for open science use, and celebrated as an active, reflexive cultural layer (See above). Goals are left to teams to identify and activate for themselves.

All biographies of "notable scientists" spend a great amount of their content describing the onlyness of these individuals (without calling it that). Every "genius" you read about is someone who managed to tap into their onlyness and find insights into infinite play (which is commonly referred to as a "serendipitous" event). Every one around them-these individuals are nearly always surrounded by colleagues - contributed to these conversations. They need to noted too.

\section{Science organizations create prizes that fail to capture the onlynesses of teams and collaboratives}

"In the end, it cannot be doubted that each of us can see only a part of the picture. The doctor sees one, the patient another, the engineer a third, the economist a fourth, the pearl diver a fifth, the alcoholic a sixth, the cable guy a seventh, the sheep farmer an eighth, the Indian beggar a ninth, the pastor a tenth. Human knowledge is never contained in one person. It grows from the relationships we create between each other and the world, and still it is never complete" (Kalanithi 2016).

No scientist has ever refused a Nobel Prize. (NOTE: Two people have declined this. JeanPaul Sartre did, because he was Sartre. And, Le Duc Tho did, because his award was shared with Henry Kissinger, who supported bombing Hanoi during Christmas.) Learned societies 
and professional academic groups offer a wide range of honors that scientists gladly accept (usually along with travel support to a meeting they would like to attend). Other honors come directly from universities or funding agencies. All of these honors fatten résumés and grease promotion portfolios around the planet.

Scientists crave the economic support they need to do the work they love. If they can translate honors into cash, they will do so. And yet, should these particular honors stop being awarded, there is little to indicate that science would be less rewarding or less able to track the value of science work. Open science offers to expand opportunities to find and use great science. New methods of acknowledging the work of scientists and teams, and also the provenance of research can replace or enhance how scientists get connected to each other through their work.

Great work in open science can be found anywhere on the planet, and also within any team working in an open-science organization, small or large. The gifts of new knowledge are freely shared, but they also obligate others to pay attention. applaud their value, and scorn those who seek attention for their own finite game. This is central to the culture of demand sharing. Recognize the work. Applaud the teams, the history, and the ideas. Show appreciation for how this knowledge is shared, without needing to pin this discovery on an individual scientist.

"Scientists are like those levers or knobs or those boulders helpfully screwed into a climbing wall. Like the wall is some cemented material made by mixing knowledge, which is a purely human construct, with reality, which we can only access through the filter of our minds. There's an important pursuit of objectivity in science and nature and mathematics, but still the only way up the wall is through the individual people, and they come in specifics-the French guy, the German guy, the American girl. So the climb is personal, a truly human endeavor, and the real expedition pixelates into individuals, not Platonic forms. In the end it's personal, as much as we want to believe it's objective" (Levin 2016).

\section{You can help your students grow their onlyness too}

Growing and tapping into your onlyness is not just a trait that the best open scientists share, it's a trait that open science needs to support, to grow, and to reward. Open science is the common work of millions of un-common individuals. The education of an open scientist requires a healthy dose of infinite-game training, the unleashing of purpose and imagination, and courage and caring in service of collective knowing. In the end, it is the research goal that becomes the teacher, and every scientist plays this alone, even within their team. Each scientist contributes the difference they have cultivated in their own insights to the collective knowing of their team. 
To help your students develop their onlyness requires that you promote in them the courage to be essentially different from those around them, and also the honesty and humility to recognize the onlyness of others. Mainly you do this by working on your own onlyness and showing how this matters. You cement your knowledge on the wall of science, daring your students to step up to a new level through you. You share conversations with them that provoke responses only they can provide. Be open to learn from their knowing.

Several authors in the past decades-from Foucault and Illich to Robinson and Godinhave pointed out that factory-style schooling diminishes onlyness (without calling it thus) in favor of standardized understandings and personal disempowerment. Open science will need to also work on the cultures of learning and teaching science. Like practical wisdom (See: The practical wisdom of doing science), onlyness needs to be exercised for it to grow.

Merchant's work on onlyness was designed to help companies become more creative and innovative through the collective conversations of their employees, powered by an organizational culture that welcomed each of them to belong to these conversations in their wholeness. What she offers to businesses holds true for the academy, and most particularly in the academy, where onlyness powers great science through networked collaborations.

- Many thanks for Nilofer Merchant's comments on a draft of this section. 


\section{0 things every open-science culture-change agent needs to know about.}

Here are the 10 things you need to know about to be an open-science culture-change agent. Pick the ones you want to challenge yourself to master.

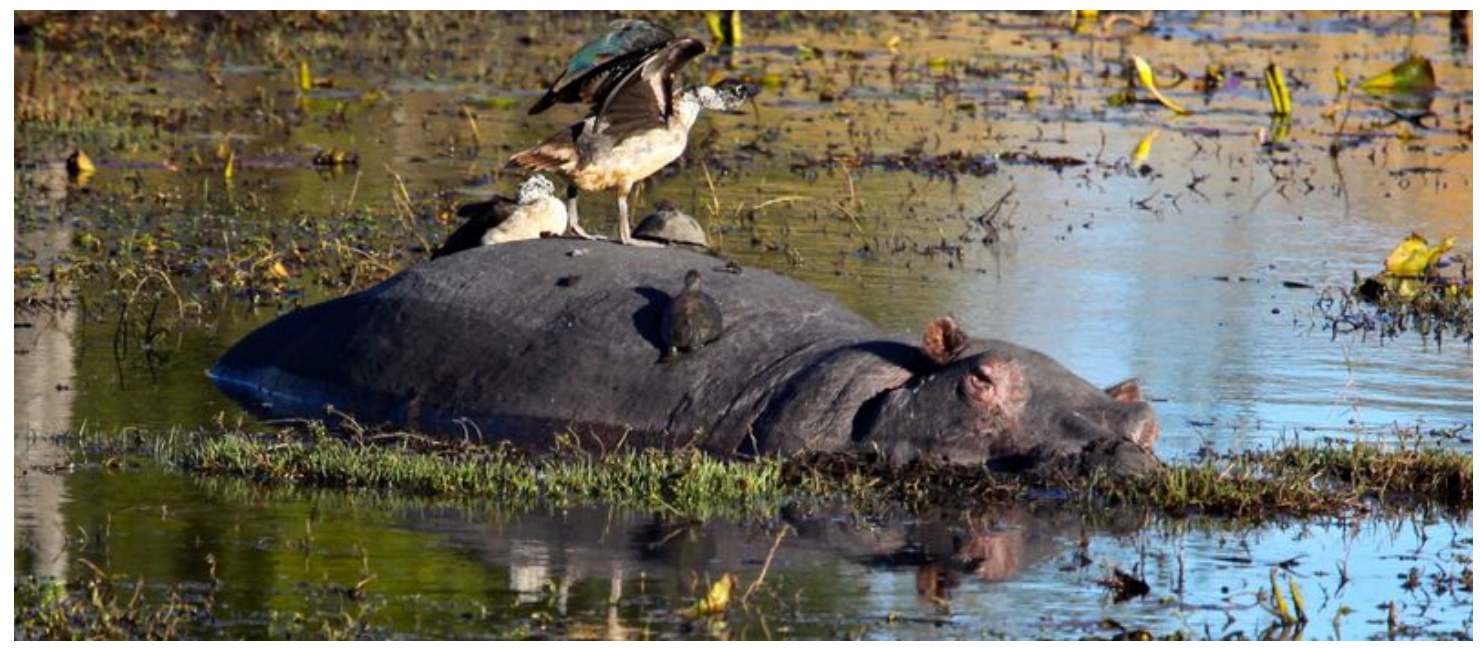

\section{Open science culture starts with the logic of demand sharing:}

This is the same logic used to teach science in classrooms: knowledge gains value when it is shared. The more it is shared the more it is worth; the faster it is shared the greater its impact; the wider it is shared the better the chance that someone else will improve upon it, and share this improvement back with you.

\section{Intellectual humility is integral to open science:}

"The humility of scientific genius is not simply culturally appropriate but results from the realization that scientific advance involves the collaboration of past and present generations" (Merton 1973).

Here are some aspects of humility and reasons why this is a great fit with open science, and a powerful agent against bullshit prestige (Moore, et al. 2017) and narcissism (Lemaitre 2015 ) in the academy. Tangney (2000) constructed a working definition of humility, one that is not simply philosophical, but also informed by social and interpersonal circumstances. This definition rejects humility as a psychological weakness, instead, humility demonstrates a range of abilities highly valuable in the conduct of science. According to Tangney, humility has five elements:

A. the ability to acknowledge mistakes and shortcomings;

B. openness to perspective and change; 
C. an accurate view of the self's strengths;

D. ability to acknowledge and experience life outside the direct consciousness of the self; and,

E. the ability to appreciate the worth of all things.

As an open scientist (or just someone who wants to do science really well), you might consider how to develop all of these capabilities. You acknowledge your mistakes in order to learn new facts; you broaden your perspectives on your topic to achieve a wider level of understanding; you evaluate your own skills to discover where you must improve your methods; you journey into the unknowns in your field to stretch the envelope of our knowledge; and you reserve judgement on the work of others long enough to fully grasp their meanings. Carl Sagan notes:

"In science it often happens that scientists say, 'You know that's a really good argument; my position is mistaken,' and then they would actually change their minds and you never hear that old view from them again. They really do it. It doesn't happen as often as it should, because scientists are human and change is sometimes painful. But it happens every day. I cannot recall the last time something like that happened in politics or religion" (Sagan 1987).

You also give others more attention and respect. This does not mean you respect yourself any less. You just learn to step around your ego to see others and their work as more valuable. Recent research has found that intellectually humble individuals may acquire new knowledge better than others (Krumrei-Mancuso, et al. 2019). Also note: "only humility can navigate complexity" (Fred Kofman <http://www.youtube.com/watch?

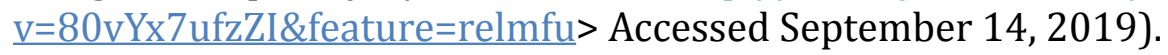

Humility helps you learn. Humility enables your research. You are a scientist: you have the freedom to be humble about it. It's not modesty. Nobody is asking you to be modest. Think of it more as "hum-ability".

In Aikido, humility becomes hum-ability. Open science is the same.

\section{Intentional kindness is the platform for open science culture:}

"The power of happiness, kindness and humility in the competitive academic environment is underrated, but I firmly believe that they are a force for change for the good of scientific practice. In my opinion, widespread application of these principles could vastly improve the quality of life of scientists and university professors worldwide" (Maestre 2018). 


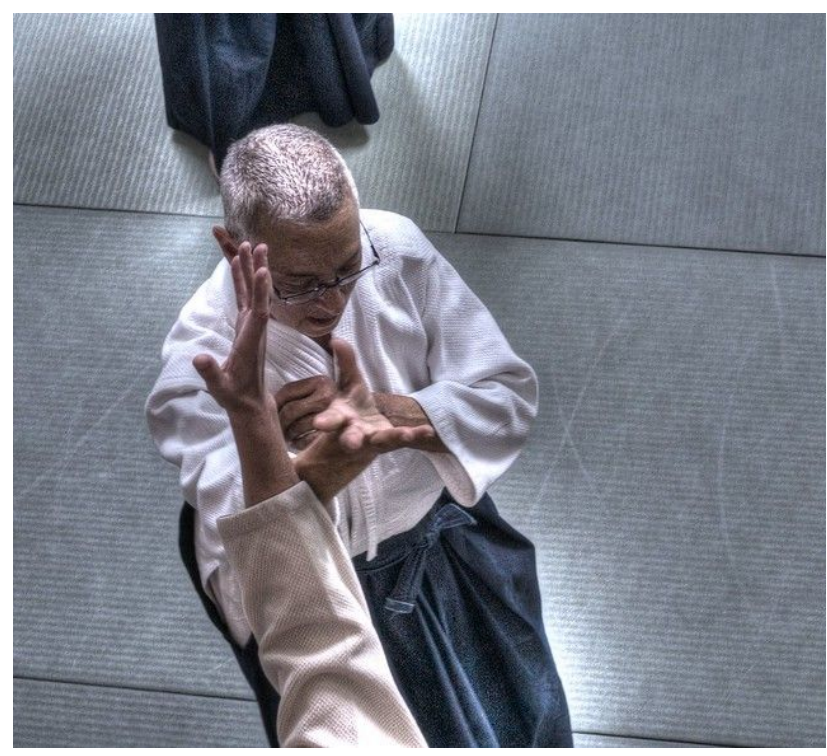

mean weakness.

Kindness in open science (end elsewhere) begins with intention. Intentions are themselves colored by culture. Culture provides a layer of shared meaning/ learning that helps us discover and interpret and map shared meaning as intended. The same conversation with different intentions can be a kind, caring dialogue, or it can be a cruel interrogation. The cultural values you bring to your open science organization can assemble the meanings that add clear intentions to acts of kindness, and to the generosity that all science requires. Just as some institutional cultures today - and inside the academysupport bullying and demeaning actions (NAS et al. 2018). Note: kindness does not

Shared kindness is a platform that lifts open science up to new potentials for sharing knowledge. In the academy, kindness is a radical form of courage. "Everyone here is smart. If you want to truly distinguish yourself: be kind" (From Anne Galloway: <https:// twitter.com/annegalloway/status/438412389319319552>).

Kindness flows from a concern for the whole science community and the planet, not just your own lab or students. The best teachers are already kind in their classrooms. Bring that kindness to your research too. Don't be that one asshole who makes others stop sharing. Kindness is not optional.

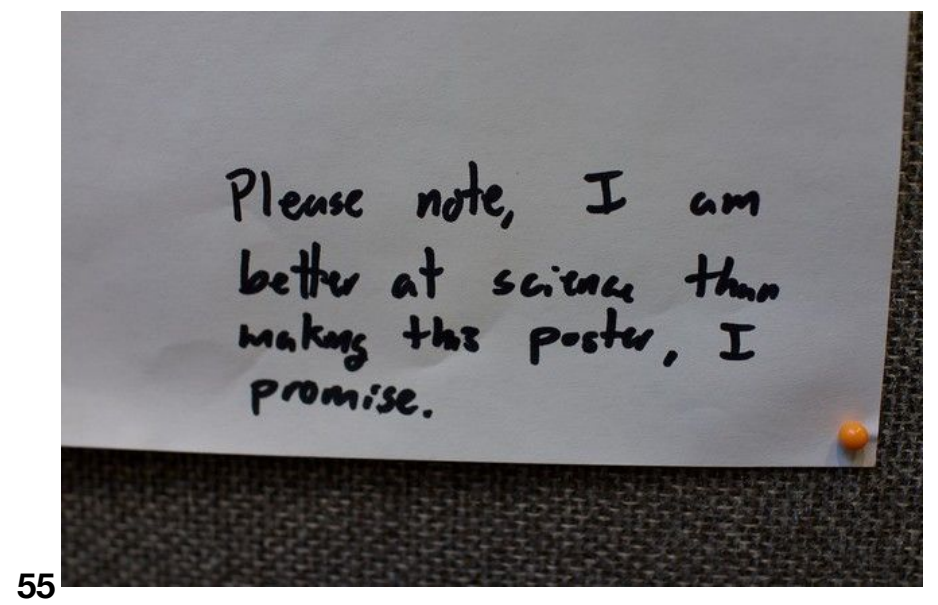

4. Open science means really open:

Open science may have started by opening up paywalled publication workflows, but it only succeeds when open extends back through the whole 
research process. Open is a manner of doing research that seeks to reveal as much of itself as it can or might, to promote shared knowing and reproducibility. Open is a transparently governed and democratic workplace in your organization. Open is open across the planet.

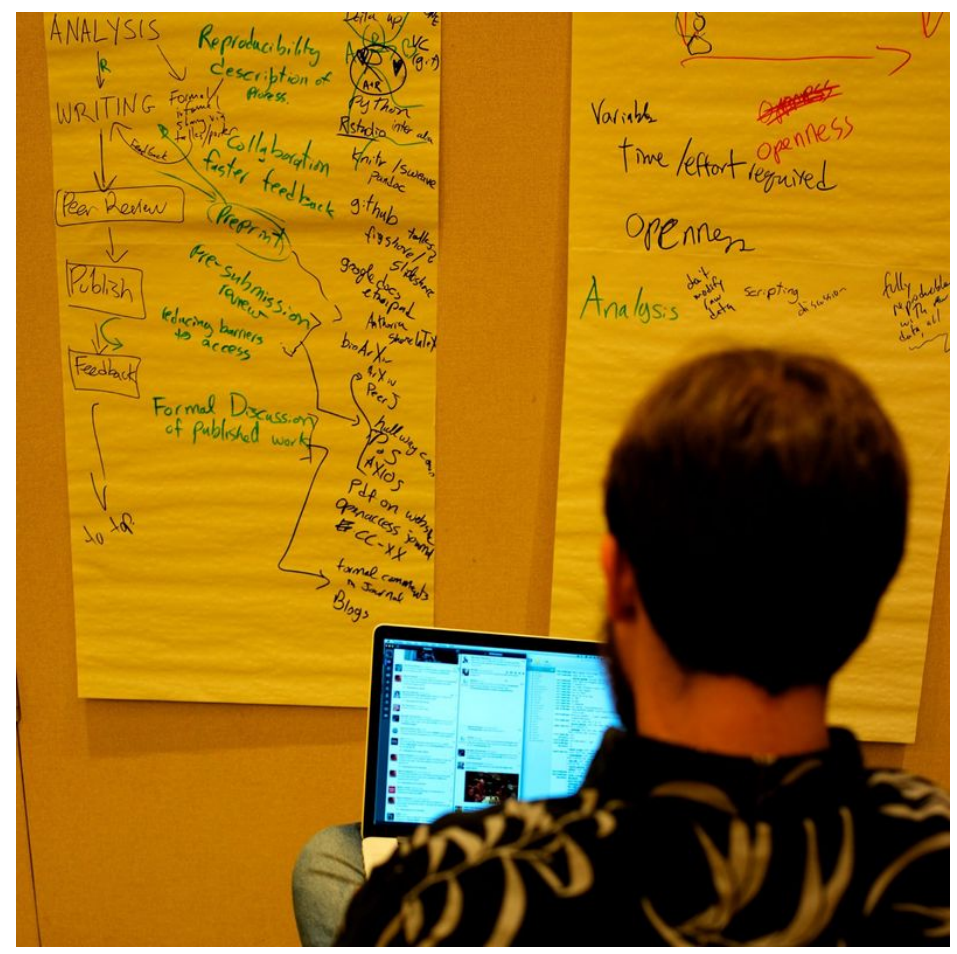

\section{Open-science culture change starts with you:}

Now is the time for you to lead your own open-science cultural change project. When you look around, you might be dismayed by the (dead) weight of organizational culture in your workplace. You can start small, and you can recruit others. The goal is to get back to the way science is meant to be pursued: infinite play against intractable unknowns, to squeeze new knowledge from observations and information.

Remember first that leadership means humble conversations (Schein 2013), fear-free interactions (Edmonson 2019), and democratic participation. You provide the compass-an informed open-science perspective-not a map. You and your colleagues are on a new learning curve toward a workplace where the only fear you find is the joyful thrill of playing with nature and data to unlock new insights. Open science needs you to find this kind of leadership inside and bring it to the academy. 


\section{Open science culture is learned:}

You learn culture just like you do science, only you started early on, and without knowing this. That's what this handbook is for. Disney and the Boy Scouts have been conscious, intentional, culture-learning organizations for decades. So too has the US Navy (for example), and your elementary school. This Handbook and hundreds of web resources are available help you discover more about open science and how to be an open scientist.

Culture is not just some subliminal vibe that you soaked up somehow (although you did a lot of culture learning really early on, and it seems like it was just soaked up). You are an adult. You are now responsible for your cultural behaviors. You can bring your focused intention and behavioral skilling to the goal of becoming more open each day. You succeed as an open scientist (and, in some fashion, as a person) by being more open today than you were yesterday.

\section{Open science culture is an on-going conversation:}

Make it a point to talk and question others about open science culture. The more people who talk the talk, walk the walk, and share what they value most, the better science will become. As John Wilbanks once said: 'the opposite of 'open' is not shut. The opposite to open is broken." Share your open science practices and stories. Keep talking with one another as you build common agreements.

\section{Open science culture must be transmitted:}

Teach your students to be open scientists. Talk to you children about how science really works in the open. Talk to their science teachers about the benefits of open science. Be an open-science mom or dad. Don't have kids? Make sure the freshmen in your class know the difference between old-science and open science. The next generation of open scientists will need to assemble their own cultures. You can give them a head start.

\section{Open science means open to all:}

Not just to the "Republic of Science," to the long-tail and beyond. Publication works when anyone on the planet can find your knowledge and share theirs with you. Do not worry; technology will help provide filters to keep you from drowning in information you do not need. Technology is one side of being open. Culture is the other side. The entire planet gets into the act at some point. 


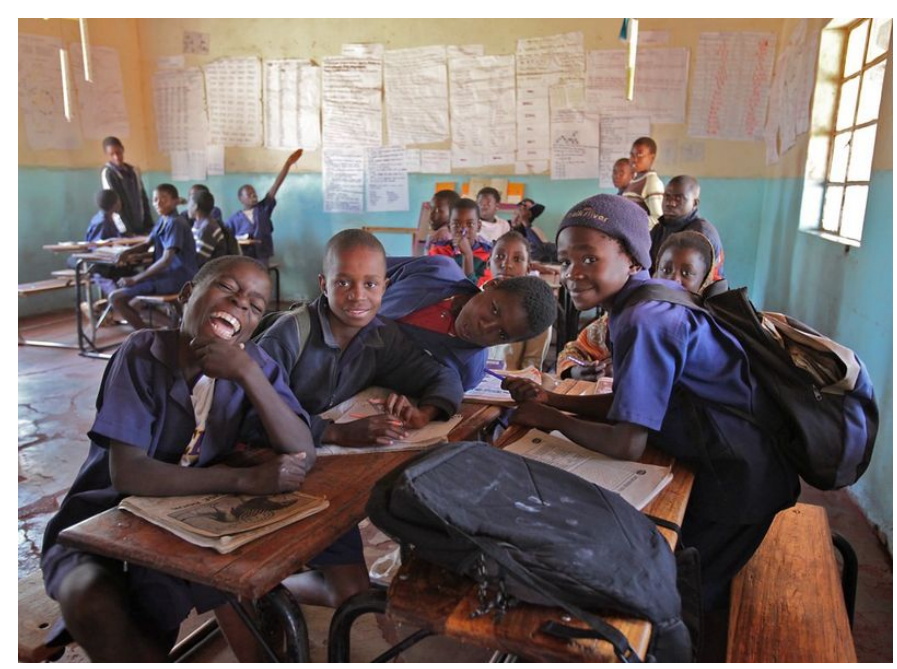

10. Open science culture will become your culture too:

You get to grow your own personal virtues aligned with the shared virtues you use in your work. You get to add passion (and nuance too) to how you realize your own cultural flavors within your various social/workplace groups. Open science wants as much of you as you care to bring to it. You can take and carry away as much open science culture as works for you. You can own your unique style of open science. Grow it. Show it off. Add new thoughts to the mix. Make a ruckus with it.

\section{Fitting into the norms of your open science workplace}

Organizations that grow their own cultural intentions and shared purpose demand more cultural/personal alignment of their members than those that do not. This is a cultural "bargain" the organization makes with its members/employees. This does not mean that the organization is oppressive, but it can feel that way if the member does not also benefit from this bargain.

As a student or faculty/staff, when you join a team that holds shared cultural norms, you are forced to reexamine your own cultural norms:

1. You can fit the organization's norms into your own: learn new intentions and meaning that are in alignment with those of the organization-grow into the organization's shared values and begin to care about these as you do your own values; or,

2. You can argue for different shared norms that are more aligned with the ones you hold as an individual. Try to convince others that different values are better. Should your arguments fail, you may need to either quit or be ready to get fired-or admit you were wrong and fit these norms into your life, or; 
3. You can pretend to celebrate the organization's norms and continue in silence to operate through your own orthogonal "principles" (until you get found out). The last choice is corrosive to the organization's purpose and culture. You are better off working somewhere else. They are better off if you leave.

Basically, when it comes to organizational culture, you can either fit in, add your own ideas to the mix, or pretend to fit in. Pretending to fit in works against the organization. Fitting in without reflection limits your ability to own the culture. So, step up and challenge any norm that doesn't seem optimal for you. If your organizational culture doesn't let you add your voice, it doesn't deserve your respect. However, if you learn you cannot fully participate in the practices expected of team members, you might want to work elsewhere. Norms and principles are open to interpretation in their practice, and enable skilling and virtuosity, like any cultural practice. The idea that you need to "be more like us" to express your own individual principles may seem counterintuitive. When you are reflexively engaged in the ongoing conversation about your organizational culture, you are creating the meaning for "like us". You own your own part of this. You can belong, and not just "fit in." 


\section{Talking Virtue and Values in Open Science}

\section{How to forge statements about your organization's open science culture}

“...when we institute prosocial or cooperative norms, they become self-reinforcing over time. The systems become dynamic: The more we practice cooperation, the more we believe in the virtue of being cooperative. These changes, of course, make the problem of sustaining cooperation easier" (Benkler 2011).

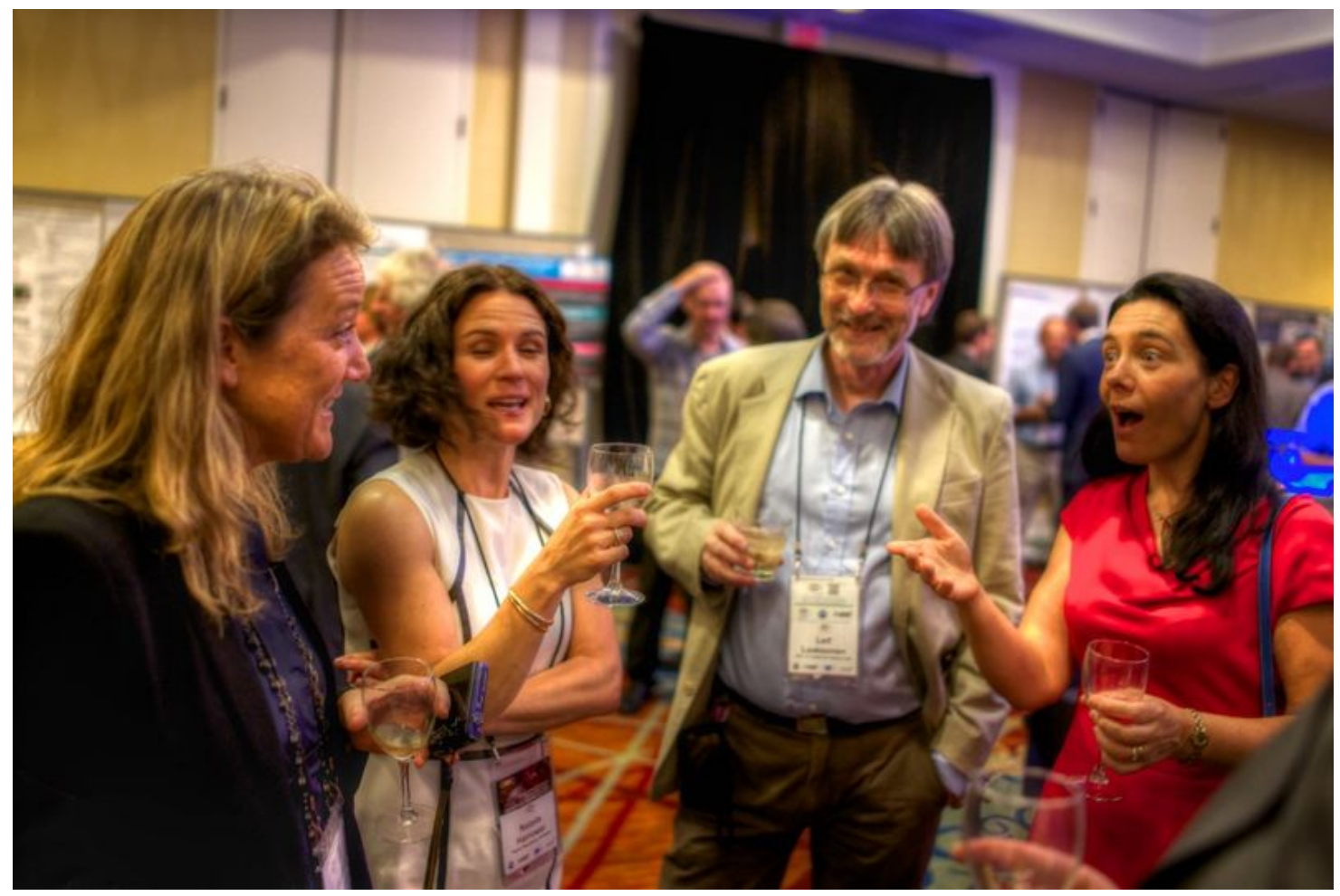

Find ways to talk about open science at your meetings

\section{Strategies, norms, and rules}

The open scientist carries a tool-belt of cultural practices that can help change the cultural habits for her organizations. Many of these tools are discursive: they happen when open scientists converse and discuss open science with their peers. Eventually, some of these conversations end up with decisions around statements that are meant to guide organizational actions.

Above, you were introduced to values, virtues, principles, and freedoms. What kinds of shared statements can you build in your organization to articulate these? This bit of the 
Handbook gives you a "grammar" for statements that should help you in your endeavor as an open science culture change agent. Statements are important, but always remember that changing behaviors is what changes attitudes. You will also need to "walk the walk" for culture change in your organization.

You've often heard about strategies, norms, and rules, but you might not know which to apply in your situation. What are the differences and similarities between these? These discursive tools are all are forged as mutually agreed-upon statements. To ratify the "agreed-upon" part, it helps to have a way to register agreement, some platform for governance, or a simple way to register a vote. The statements can then be delivered as shared agreements in conversations, offered in speeches, tweeted and retweeted, and discussed and reformed through further deliberations.

Shared strategies, norms, and rules announce and define how the organization's actions match its shared values and common interests. Culture change agents can nudge their organizations to consider new strategies, norms, and rules that can promote open science by supporting new behaviors that spawn new attitudes.

\section{A grammar for organizational norms}

One helpful system you can use to know how to craft statements that become strategies, norms, or rules was devised by Elinor Ostrom (of commons governance Nobel fame) and Sue Crawford (Crawford and Ostrom 1995). This has since been expanded by other economists and social scientists. Crawford and Ostrom proposed a grammar for organizations (in analogy with a Chomskyan syntax for language) based on five descriptors. Statements about organizational strategies, norms, and rules will contain a certain selection of the following grammar:

$A=$ Attributes of the actors to whom this institutional statement applies.

$\mathrm{D}=$ Deontic content of the statement, specifying which actions may, must, or must not be undertaken by the relevant actor.

$\mathrm{I}=$ Aim or Target denotes the action or outcome to which the action in question in to be applied.

$\mathrm{C}=$ Conditions under which this particular statement is deemed appropriate or relevant for application.

$\mathrm{O}=$ Or Else specifies the actor or actors to whom is given the responsibility of imposing sanctions on those who fail to implement the statement as intended.

(From: McGinnis 2011)

Let's unpack these five parts of statements to show to they can be useful for most of the circumstances an open scientist might face: 
$A=$ Members of the group, or some sub-set. For example: all tenured professors in your college, all members of your professional association, all post-docs in your laboratory. Attributes are used in every strategy, norm, or rule statement, unless there is an implied "everyone."

$\mathrm{D}=\mathrm{A}$ dutiful attention to either performing a task or avoiding a task; or a permission or prohibition about the task. For example: "you must make your research data public whenever possible"; "everyone should put their published work into an open repository as soon as they can"; or, "we should boycott for-profit publishers that don't allow open data mining of their corpus." This is what separates rules and norms from strategies.

I= The aim or goal of the action of the statement. For example: "In order to build transparency and reproducibility, you must make your data public"; "To increase discoverability..."; or, "With a goal of pressuring them to open up their corpus...;"

$\mathrm{C}=$ Conditions. Some statements apply to specific circumstances. When no condition is listed, the circumstances are, by default, universal to the group's activities. For example, a group may create a statement (code) about proper conduct. They may have rules for on-line conduct, and different/additional rules for conduct at conferences. In terms of open science, open data statements might include privacy conditions when the data were donated by individuals. Conditions pin the statements to real-world circumstances.

$\mathrm{O}=$ Or else. The punishment/sanction for not following the statement. As you'll see below, this part is what separates a rule from a norm or a strategy.

\section{Some Statements of Open-Science Strategies}

\section{Strategies use AIC grammatical parts}

What is good to remember here is that information about open science is strategic, as it informs the choices of others. A strategy means a statement about a practice. The statement may be experimental, say, a suggestion about a new technology. It may offer a good practice to follow. Such statements add to the inventory of useful information in support of openscience practices and virtues, or they refute statements made that may mischaracterize open science.

Here are some examples:

"As an open scientist, when you are asked to review an article, check to see that the article will be openly available."

"Members of (insert name of a professional academic association) can add their publications to our open repository." 
"Tenure decisions should not use journal impact factors."

"Take the trouble to see if there is an open alternative text book for your class subject."

"Maintain the provenance of data, so that others can reuse this more effectively."

\section{Some Statements of Open-Science Norms}

Note here: your organization will be crafting and modeling its own norms, and statements about these. Norm statements become real norms when they describe default behaviors (people like us open scientists do things like this), not aspirational behaviors, within a community. Of course, that requires work and time and governance to accomplish. Without real democratic governance processes to grow these, norms resemble rules laid down by those in power. Norms add the element of dutiful attention [AIC+D]. So they are strategies that are actually practiced. When spoken, they take strategy statements and add a "we...." Norms are strategies that all of us do all the time here, in this community/organization.

You can help uncover and then recode norms by opening up conversations about "the way we do things here." Remember that the impact of these norms will rely on changing/ inventing practices and processes that align with them.

Here are some examples:

"At our university, we only publish in green open-access journals."

"Scholars in our discipline always preregister our research to improve transparency."

"Our lab never uses journal impact factors at all, ever."

"Every panel in our society's conference has at least two under-represented scholars."

"Everyone hired to teach at our college, from senior professor to new adjunct, gets a vote on every issue."

Norms are cultural practices that become "normal behaviors" by being widely shared among a community (e.g., your college). They are the community's vehicles for announcing "the way things are done here." Norms can become tactic knowledge, and can be learned through emulation, instead of instruction. When enough of a community do the same thing the same way, or talk about what they do the same way, this "way" is a norm. Norms describe common practices that express virtues (valorized cultural/social behaviors that carry ethical meaning) and shared principles (valorized value positions).

\section{Some Statements of Open-Science Rules}


Rules are like weaponized norms. Rules add the "or else" to a norm (AICD+0). While it may be easy to write the "or else" phrase, this is a more complex aspect to use in practice, since it requires that the organization has a method to enforce the sanction, and it puts the violator into a new category of attribute (e.g., as someone who may no longer be eligible for election to group leadership roles, or who is kicked out of the group entirely). It also means that others in the group may then have a new duty to act differently to the violator (e.g., not reviewing their work, not hiring them). So this statement will be connected to other statements that spell out the procedures for the sanction. Again, the "or else" clause is what separates a rule from a norm or a strategy.

Here are some examples:

"All proposers will add a data management plan to their proposal." [Implied: "Or else your proposal will be rejected.]

"Behave professionally. Remember that harassment and sexist, racist, or exclusionary jokes are not appropriate." [Or else you will be asked to leave the conference.]

"We do not tolerate harassment in any form. Discriminatory language and imagery (including sexual) is not appropriate for any event venue, including talks, or any community channel such as the chatroom or mailing list." [Or else you will be excluded from on-line participation.]

"By 2020, the final published version of articles will be made immediately Open Access (with a CC BY licence) and copyright retained by the author." [Implied: "Or else you will not be funded.]

Rules are valuable when used by organizations against bad actors in the academy. Codes of conduct only really work when there are consequences to violating these. (See: The Carpentries Code of Conduct Enforcement Document. Accessed May 29, 2019) Rules that set behavioral boundary conditions are necessary to help re-ground a culture that has become toxic. Your organization can signal that a culture change has happened by ruling out formerly accepted behaviors. Rules are much less valuable for announcing and enforcing good behaviors. For example, Clegg and Roland (2010) warn that rules concerning "due care" in education can interfere with the practices that carry authentic kindness.

\section{Enforcement by leverage}

The easiest way to enforce a rule is when you have economic leverage over the actors in the attribute part of the statement. At that point you may not need to create the "or else" phrase, because it is implied. A funding agency can create a rule by stipulating a task as part of applying for funding. "All proposers (this is the A statement) must (this is the D 
statement) submit a sustainability plan (the I statement) with their proposal (the C statement)." The 0 statement, "or we will not review this (favorably) for funding" is implied.

Open data efforts may push for funding agencies to enforce open data rules rather than for societies or universities to engage their members to do so. Enforcement by leverage is often viewed as easier than hosting democratic governance to manage a situation; and, yes, it is certainly less cumbersome. But rules also become social obstacles as they foster rulebending behaviors, and cannot alter the intent of the individual scientist, nor encourage voluntary normative behaviors. Voluntary normative behaviors are informed by shared virtues and principles.

The Handbook looks to give you the tools you need to work with your colleagues and change your organization's culture. Knowing how to fashion strategy, norms, and rules enables you to avoid creating unnecessary rules, or mistaking strategic statements for norms. You will populate these statements with the values (principles, etc.,) that you've already determined.

\section{Don't forget the narrative}

Finally, you need to add some narrative framing to your statements to reveal their dynamics. Open science offers us new choices, new knowledge tools, and new ways of working together. Framing your statements as narratives invites others to join in. John Hegel $(2018$, Accessed September 3, 2019) notes, "a narrative is a call to action directed at the listeners, stressing the role that they can and need to play in resolving the narrative." 


\section{Fierce Equality}

"The Ju/'hoansi people of the Kalahari have always been fiercely egalitarian. They hate inequality or showing off, and shun formal leadership institutions. It's what made them part of the most successful, sustainable civilisation in human history..." James Suzman in The Guardian, October 2017

"Open scientists in the academy are fiercely egalitarian. They hate inequality or showing off, and shun formal leadership institutions. It's what made them part of the most successful, sustainable intellectual forces in human history..." Hopeful message from the near future.

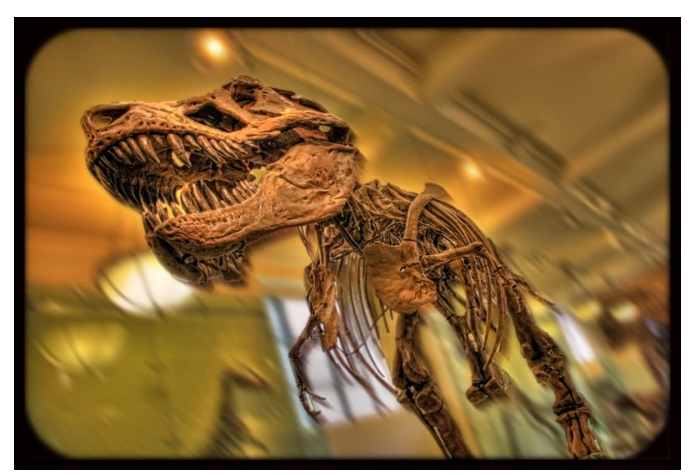

This is Sue (true). She really loves open science (not as true). Fierce equality is universalism with teeth. Photo credit: Daniel Mennerich on Flickr. CC by-nd-nc 2.0

\section{Why fierce equality matters to the academy}

The academy needs equality, and not just the word. It needs normative, active, celebrated, fierce equality. It needs this first as a corrective to the twisted incentives of the past century of perversely cumulative advantage (DiPrete and Eirich 2006). It needs this as an open door for scientists in the south who have been locked out of conversations. It needs this to ground a new operating logic that does not permit the hiring of temporary faculty at penurious wage scales. It needs this to repair so many years of gender inequality. It needs this because the best science comes from a requisite variety of knowing that is all inclusive. Here we will explore this need.

\section{The Academy Lacks Fierce Equality Today}

The contrast between what fierce equality would look like in the academy and what you will find today, looking around your university, your discipline, your career (and those of your students), is probably striking. It was never supposed to be this way. 
Science was meant to be rigorously inclusive. Merton (1942) used the term "universalism" to describe the foundational democratic norm of science (one of four norms, also the norm that most tended to be "deviously affirmed in theory and suppressed in practice" (ibid)). Universalism meant, and still means, that scientific discoveries can be made anywhere, by anyone. New discoveries are validated by the community (usually through replication). Their discoverers have equal standing in the "republic of science"(Polanyi 1962) without the need for additional institutional or personal validation. There are pragmatic constraints about proper methods and reporting that add a higher threshold to enter the cadre able to do and report science. Fierce equality ensures that crossing this threshold is not anybody's exclusive privilege.

\section{Cumulative Advantage}

The suppression of universalism has several sources, including the external logic of neoliberal markets. Another factor is what Merton termed the Matthew effect. The Matthew effect describes all the ways that advantages accrue to a few individuals and are, simultaneously stripped from the rest. "Differences in individual capabilities aside, then, processes of cumulative advantage and disadvantage accentuate inequalities in science and learning: inequalities of peer recognition, inequalities of access to resources, and inequalities of scientific productivity. Individual self-selection and institutional social selection interact to affect successive probabilities of being variously located in the opportunity structure of science" (Merton 1988).

Cumulative advantage has well-studied institutional and geographic features, which lead to advantages and disadvantages in hiring, funding, and publication. Despite a raft of entitled pronouncements to this effect, the academy is not a meritocracy; or else, it's a terrible example of one (Morton 2019 (Accessed May 30, 2019); Standing 2011; Emkhe 2018 (Accessed May 30, 2019); Way, et al. 2019; Harmon 2018 (paywalled, Accessed May 30, 2019); NAS Committee on the Impacts of Sexual Harassment in Academia 2018). Academia is an informally reproduced aristocracy. It was never supposed to be this way; apart from the fact that it's been this way for a long time. Which is why fierce equality matters.

"It is the priority rule which identifies the author of the discovery as soon as he or she publishes and which thus determines the constitution of "reputation capital," a decisive element when it comes to obtaining grants. "The norm of openness is incentive-compatible with a collegiate reputational reward system based upon accepted claims to priority' (David 1998). The priority rule creates contexts of races (or tournaments), while ensuring that results are disclosed. It is a remarkable device since it allows for the creation of private assets, a form of intellectual property, resulting from the very act of foregoing exclusive ownership of the knowledge concerned. Here the need to be identified and recognized as the 
one who discovered forces people to release new knowledge quickly and completely. In this sense, the priority rule is a highly effective device that offers non-market incentives to the production of public goods..." (Foray 2004).

\section{Hyper-competitiveness (and funding)}

Hyper-competitiveness at the institutional and personal level "crowds out" (Binswanger 2014) science's intrinsic motivations and promotes quantity over quality, "bad science" (Smaldino and McElreath 2016), and marketable formalism over research needs. Worse, it crowds out scientists who refuse to play the bullshit-excellence game required by the gamification of reputation in the academy. The "priority rule" of discovery in the academy is really just a method to gamify episodes in the lives of research teams for the reward of individuals against the benefits of discovery for science and the world. Foray does not claim that personal recognition is required for scientists to rapidly release research results; or that personal ownership of the knowledge contributes to knowledge sharing. "On the contrary, the tournament contexts created by the priority rule, as well as the size of related rewards, tend to encourage bad conduct" (ibid). The production of the public goods of science in these circumstances has become sub-optimal, feeding the goods of reputation metrics instead of the benefits of open demand sharing across the academy.

Competition also feeds the Matthew effect: "[I]ntense competition also leads to 'the Matthew effect'...this competition and these rewards reduce creativity; encourage gamesmanship (and concomitant defensive conservatism on the part of review panels) in granting competitions; create a bias towards ostensibly novel (though largely nondisruptive), positive, and even inflated results on the part of authors and editors; and they discourage the pursuit and publication of replication studies, even when these call into serious question important results in the field" (Moore, et al. 2017). Science loses on all scores.

For science, hyper-competitiveness is a race to the bottom that so many institutions are fighting to win using arbitrary metrics as goals. "Competitiveness has therefore become a priority for universities and their main goal is to perform as highly as possible in measurable indicators which play an important role in these artificially staged competitions" (Binswanger 2014).

\section{Fierce equality and funding}

Universities, funding agencies, and major foundations will need to construct new hiring, promotion, and funding practices that ignore ersatz excellence, pseudo-merit, and cumulative advantages. This process begins by envisioning how the outcomes of funding can be shared with equity across society, and then operationalize this vision. A recent funding model (Pluchino et al. 2018) revealed that, since talent is well distributed across 
research populations, funding researchers who have already had a success (mainly due to luck, which is another interesting finding) underfunds others with great talent, but less fortune to date. Giving every researcher access to some funding would result in greatly improved research returns on these funds. Refactoring hiring, promotion, and funding is the academy's greatest need, and largest challenge, today. Changing the core logic for hiring, promotion, and funding will be a monumental task (Smaldino, et al. 2019). Failing this task, science will continue its race to the bottom. Tossing this task onto the shoulders of "open science" is perhaps unfair: this is a wider, deeper need of science and society (Newfield 2016).

What fierce equality adds here is a new/old logic to anchor the discussions and decisions over what must come next. Like Merton, you can begin with the classic science norm of universalism; this time around, it's vigorously affirmed in practice. You will find discussions on alternative research funding schemes and tenure solutions in other parts of the Handbook. As we learned in The Work of Culture (above), the academy will need to change behaviors to change attitudes, to change practices, to change research culture toward new ways (and sources and, hopefully, new amounts) of funding.

\section{A closer look at fierce equality}

What is "fierce equality" and how is this better than simple "equality"? You might note here that the Ju/'hoansi people, those hunter-gatherers who have practiced this for millennia, do not call their own cultural practices "fierce equality." This is how anthropologists have captured the integral role that equality has in their cultural practices, and the tough behaviors that are used to maintain this equality. Highly visible, shared cultural behaviors protect this norm against those within their group who are "bad actors." Fierce equality is equality publicly defended at every opportunity where personal or group entitlement pops up. Those who might argue that fierce equality would only work in small-scale cultural groups might want to reflect that most academic work happens in small-scale cultural groups (labs, departments, college faculties, teams).

"The more widely the republic of science extends over the globe, the more numerous become its members in each country and the greater the material resources at its command, the more clearly emerges the need for a strong and effective scientific authority to reign over this republic. When we reject today the interference of political Jr religious authorities with the pursuit of science, we must do this in the name of the established scientific authority which safeguards the pursuit of science" (Polanyi 1962).

Fierce equality means that open-science organizational behaviors: governance policies, rules, codes of conduct, plans for sharing and access to resources and to recognition, funding strategies, hiring practices, and face-to-face interactions are liable to be judged by 
how they promote equality within the global "republic of science." Fierce equality operates internally in the academy (nobody expects the rest of the world to comply), and internally in all of the academy's various organizations, each of which expresses this norm in their own self-determined governance. Every chapter in this book will talk about how open scientists can promote and perform fierce equality in their daily work.

Fierce equality challenges hierarchy in the academy. Benkler (2016/17; Accessed June 6, 2019) calls out hierarchy as a key concern when refactoring an organization: "The first is the concern with the power of hierarchy; the power within an organization to be controlling. This is the concern with the bossless organization, this is the concern with participatory governance." The NAS (2018) pointed to hierarchy as a factor in "mistreatment" in the academy. Organizational change in the academy needs to start with unchallenged hierarchies.

Fierce equality is not a luxury. It is a long-term optimization strategy for the global republic of science; an expectation that emergent capabilities for sharing, mining, mixing, and reusing science objects can only realize their potential as a planet-wide, provident scientific resource when the entire community adheres (in multifarious ways) to the norm of equality. To build knowledge-maintenance organizations that are self-sustaining across decades and centuries of time, and for the whole of the global academy, there is no more fundamental principle than fierce equality. And there is no better time than now to refactor the academy using fierce equality as a foundational principle. Fierce quality was the advanced cultural practice system that informed the behavior of a majority of humans for tens-of-thousands of years.

"This research also revealed that the Ju/'hoansi were able to make a good living from a sparse environment because they cared little for private property and, above all, were 'fiercely egalitarian', as Lee put it. It showed that the Ju/'hoansi had no formalised leadership institutions, no formal hierarchies; men and women enjoyed equal decision-making powers; children played largely noncompetitive games in mixed age groups; and the elderly, while treated with great affection, were not afforded any special status or privileges. This research also demonstrated how the Ju/'hoansi's 'fierce egalitarianism' underwrote their affluence. For it was their egalitarianism that ensured that no-one bothered accumulating wealth and simultaneously enabled limited resources to flow organically through communities, helping to ensure that even in times of episodic scarcity everyone got more or less enough.

There is no question that this dynamic was very effective. If a society is judged by its endurance over time, then this was almost certainly the most successful society in human history-and by a considerable margin. New genomic analyses suggest that the Ju/'hoansi and their ancestors lived continuously in southern Africa from 
soon after modern H sapiens settled there, most likely around 200,000 years ago. Recent archaeological finds across southern Africa also indicate that key elements of the Ju/'hoansi's material culture extend back at least 70,000 years and possibly long before. As importantly, genome mutation-rate analyses suggest that the broader population group from which the Ju/'hoansi descended, the Khoisan, were not only the largest population of $\mathrm{H}$ sapiens, but also did not suffer population declines to the same extent as other populations over the past 100,000 years.

Taken in tandem with the fact that other well-documented hunting and gathering societies, from the Mbendjele BaYaka of Congo to the Agta in the Philippines (whose most recent common ancestor with the Ju/'hoansi was around 150,000 years ago), were similarly egalitarian, this suggests that the Ju/'hoansi's direct ancestors were almost certainly 'fiercely egalitarian' too" Suzman (Accessed June 6, 2020).

\section{The academy as a gift economy}

Fierce equality opens up contributions from across the world of science, and works at strengthening the "long tail" of discovery where real diversity spawns a massive variety of intelligences and promises innovation, discovery, fresh ideas, new knowledge. Fierce equality upholds the academy as an open gift economy, with its own logic of demand supply.

As Lewis Hyde puts it: "A scientist may conduct his research in solitude, but he cannot do it in isolation. The ends of science require coordination. Each individual's work must 'fit,' and the synthetic nature of gift exchange makes it an appropriate medium for this integration; it is not just people that must be brought together but the ideas themselves" (Hyde 2009). You can check out Gifting and Reciprocity later in the Handbook. What is important here is that "the academy" or "the republic of science" - whatever you wish to call the planetary endeavor for new knowing-needs to operate as a specific type of gift economy, using Demand Sharing as its logic, and fierce equality as a core norm. An interesting tension that Hyde notes and resolves is how the academy uses knowledge (e.g., published papers) as gifts to offer status rewards, but does not actually attach this status to individuals as much as to the quality of their work and to their willingness to give this away to the scientific community. Any additional "prestige" attached to these gifts actually works against the interest of the global science community, and can be labeled a perverse effect on this.

"If you can find it within yourself to stop using conversations as a way to convince people that you're right, you will be stunned at what you've been missing. A flood of information will rush in to fill the vacancy left behind by your ego. You might be overwhelmed with knowledge, perspective, insight, and experience. You'll hear 
stories you had refused to hear because you were too busy stating and restating your case. If you enter every conversation assuming you have something to learn, you will never be disappointed.

If you want to articulate your opinion, write a blog. If you want to have a conversation, set your opinions aside, at least temporarily. You might find you never want to return to them. You may find you've evolved beyond them" (Headlee 2018).

\section{Fierce equality does not mean "all ideas are equal"}

Fierce equality is about equity of inclusion in academic life and work. It makes no claim about the relative qualities of the ideas introduced into the scientific conversation. All ideas are liable to validation and evaluations of their usefulness within their research domains. All findings are liable to interrogations of the methods and data that produced them. All scientists deserve to be heard, to be an equal part of the conversation.

Fierce equality is about erasing the dead weight of privilege, in exchange for open (as in to all, with additional recognition for contributions) knowledge collectives: cultural groups inside, outside (or both) of the current academic establishment. The goods of the academy will still be vetted; in fact, reviewed with greater transparency, fairness, and effectiveness than current peer review (Tennant, et al. 2016).

"Given the right opportunities, humans will start behaving in new ways. We will also stop behaving in annoying old ways, even if we've always tolerated those annoying behaviors in the past" (Shirky 2010).

Applying a logic of fierce equality to your organization might present a variety of challenges. Your long-standing academic organization may have settled into any number of "annoying behaviors" that are defended as traditions, or simply as "the way we've always done things." This Handbook is here to help you become a culture change agent, to kickstart the conversations that decenter pre-internet, pre-open science practices. Open science is here to offer a whole mix of "the right opportunities," so your organization can do better things and stop getting better at doing obsolete things (Dintersmith 2018).

\section{Make a vision statement for fierce equality in your organization}

A vision of the academic world operating though fierce equality is a thought experiment that many people in many academic organizations will need to do in the next decade. You and your colleagues can open up Culture Changing Activities beginning with statements about values and vision.

Here is one example of a fiercely equal, future-of-the-academy vision statement: 
We envision an academy where members openly share their most important thoughts, processes, data, and findings through self-governing commons that are intent on the longterm stewardship of resources, on the value of reuse, on the absolute equality of participation, on the freedom of scientific knowledge, on open access to common infrastructures, and the right of all to participate in discovery and of each to have their work acknowledged, if not with praise, but with kindness and full consideration.

The particulars that inform this vision might include the following:

- Widespread use of lotteries for institutional or volunteer "leadership" positions (including department chairs and some deans), with initial terms of office fairly short (just long enough to evaluate performance) and opportunities for follow-on appointments (with limits). Good service is still noted and can be another source of informal recognition.

- $\quad$ Badges - when these are openly available to be acquired - can also be used as preconditions for entering lotteries. Want to be considered for dean? Take this badge MOOC. Skilling can be acknowledged and rewarded through badges. Badging also can become a primary task for professional associations/societies, as long as the ability to acquire the badge is not made exclusive.

- The act of making one's science work objects publicly available supports non-exclusive, anti-scarcity services: open repositories, pre-prints, idea farming sites, etc.

- $\quad$ Career moments (promotion, job switching, etc.) are evaluated externally, and keyed to a record of active demand sharing, indications of non-assholish behaviors, and activities that celebrate the institutional values and norms. Also, job applications have a layer of lottery to randomize selection (perhaps between an initial evaluation and the final decision). Implementing this is tricky and will require experimentation to optimize.

- Lotteries are distributed into diversity buckets to be sure that the variety of selectees includes those who might otherwise be excluded.

- Funding gets spread out to the long-tail of the community, with an ability to/ requirement to also crowd-source the redistribution of some funds to promote work that is of widespread benefit.

- Laughing at bullshit "excellence" and at the former desire to build exclusive academic "brands." Remember it is possible to be elite, without being exclusive. Remember "Harvard"? Remember "Nature"? Smile. Recognition shifts away from individuals and institutions and to the actual work and all the teams currently adding to this, and the long history of that work. 
- Nobel—and other-prizes honor ideas shared among networks (Keating 2018). Lists of scientists across the planet who have contributed to a selected avenue of research might be assembled, mainly as a reference for future collaborations or historical records. Even as we might ridicule a government official for demanding gratitude when he was only doing his job, we need to start ridiculing those who want to claim personal credit for research results that a built on a wellspring of shared knowledge, teamwork, and luck. 


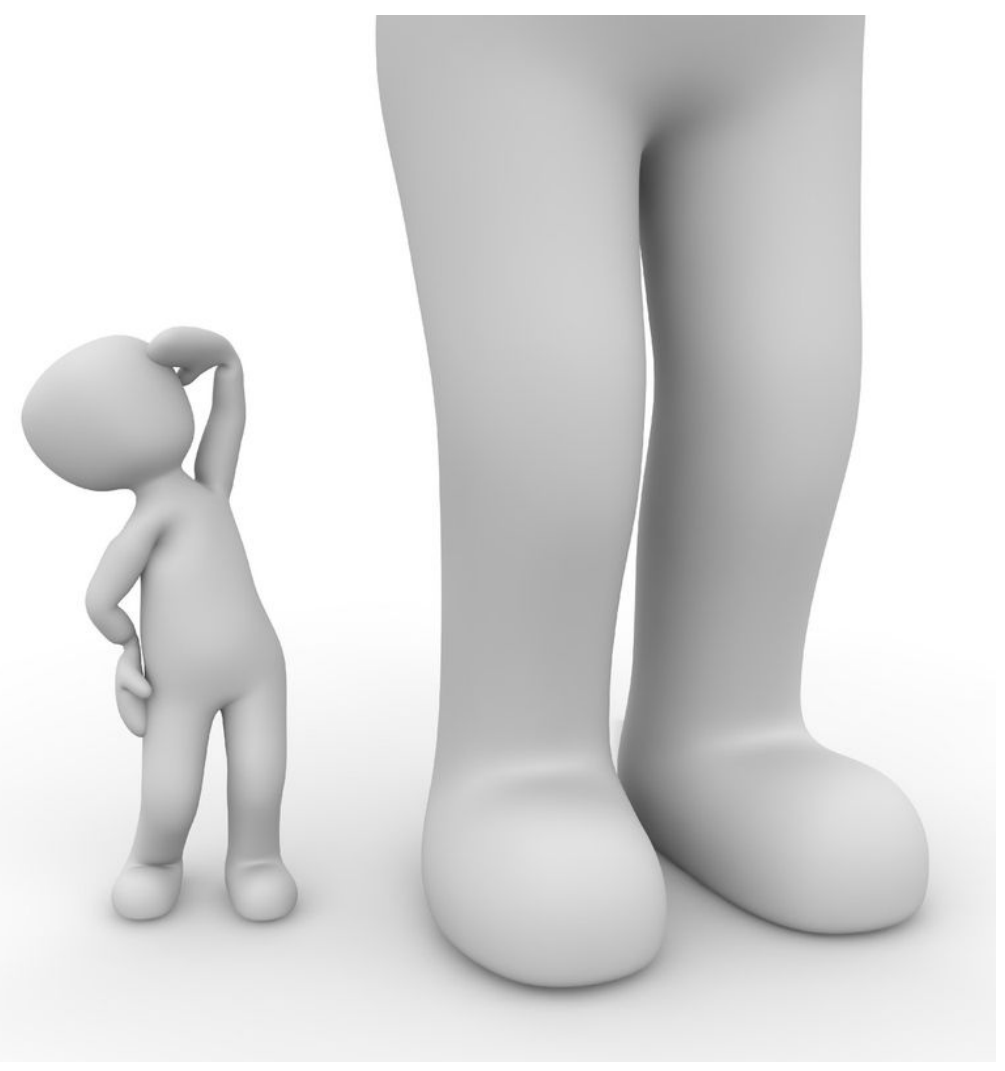

"If I have seen further it is by standing on the sholders [sic] of Giants." Isaac Newton. 1676. Letter to Robert Hooke (before they became bitter enemies). This notion was a commonplace in the $17^{\text {th }}$ Century, with the implications that even a dwarf would see further than a giant if he were standing on the giant's shoulders. (Wikipedia).

"If our team's ideas add value to the current state of knowledge, it is because we have stolen widely and well from the abundance of prior understanding surrounding us, and climbed a stairway of knowledge built by others." Modern version... no giants.

\section{It's time for science to admit that no scientist is a lone giant in their field}

One of the hard lessons for open science is to abandon the notion that "great" scientiststhose "giants" of the academy - were and are individuals of some unique and rare quality; that their shoulders tower above those of their peers, and that the optimal career goal of a scientist is to become a giant in their field. And, if you are a woman in science, while standing on the shoulders of "giants" in the academy, you can be fairly certain that some of 
them would have been intent on looking up your skirt; another reason why open science needs Fierce Equality.

In Isaac Newton, the Asshole who Reinvented the Universe (Freistetter 2018) we get a picture of Newton's brilliance as a natural philosopher, and of his serial acts of intellectual and careerist selfishness. Biographies of Newton's career and personality issues are several (See also: Clark and Clark 2001; Manuel 1979; Gleick 2004). The biographies of several of his contemporaries (Leibniz, Hooke, Gray, and Flamsteed for starters) reveal their side of the dangers of being on Newton's wrong side. But then Newton's bad behavior was not as unusual as it perhaps should have been. Rather, it was distinguished by its obsessive persistence, and made potent by Newton's position at the head of the Royal Society. Freistetter excused much of Newton's bad behavior as emblematic of an academy culture where intellection was - and still is - a cerebral variety of blood sport. He did venture that Newton would still be called an asshole if he were working today.
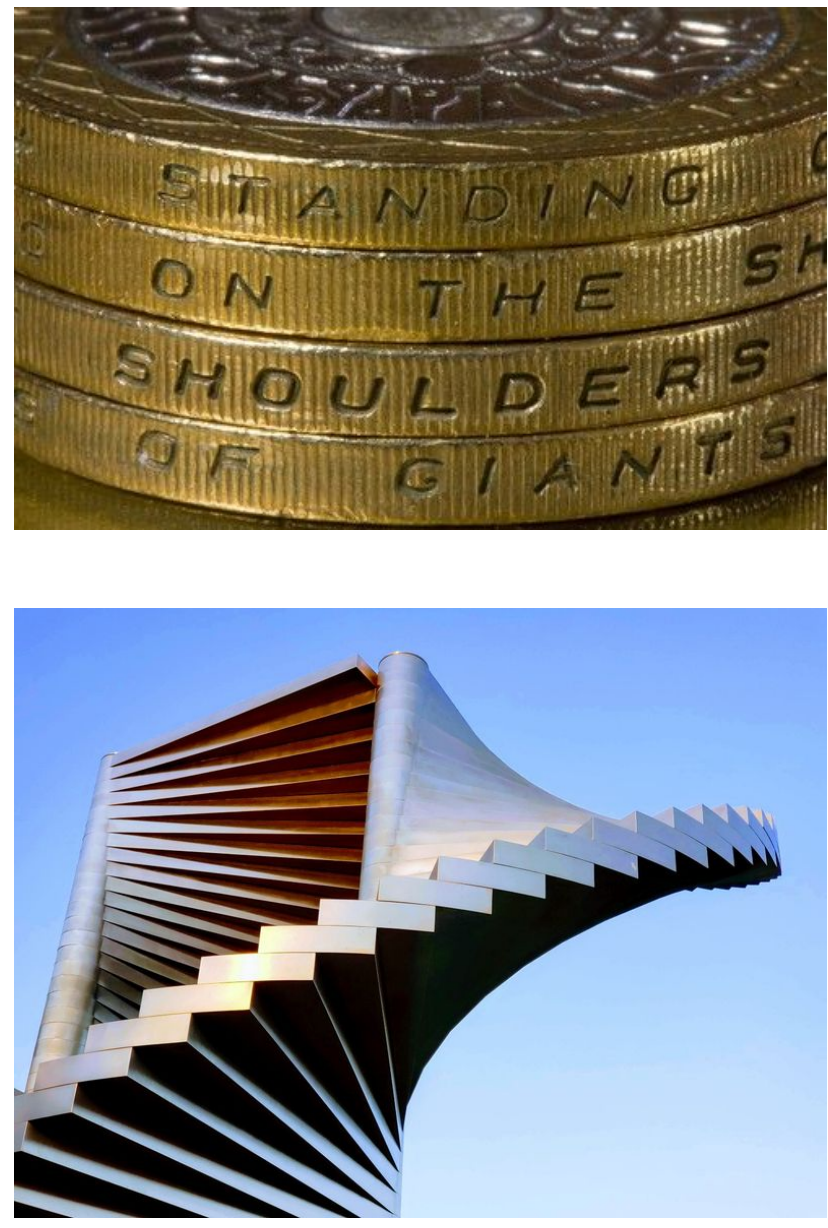

Newton's interpersonal misconduct is less of an issue here. For more on assholes, take a look at The Zero-Asshole Zone. While producing a series of astonishing research findings from his own work, Newton was soaking up ideas and credit from others, while insisting that his ideas were his alone. Apparently, we wasn't entirely serious about the whole "giant sholders" thing.

You want to get good at doing science-as a personal goal-because this leads to more satisfaction in your daily life and career, and because you can become more valuable to science by having better conversations that lead to more interesting questions and new ideas. You getting better at being a scientist should, in no manner, obstruct others on their path to getting better at this. In fact, one of the advantages of open work in science is that you can lift others during your climb up the same stairs. You 
can always grow. You can grow a larger sense of the science you are working with/on, a perception of how your work fits into the field, and appreciation for the work of your colleagues. Your primary challenge is to be better at science (and being human) today than you were yesterday.

"[B]y some measure, every important innovation is fundamentally a network affair" (Johnson 2011).

"[M] odern scholarship is based on cooperation. Ideas are not created in a vacuum. Reuse of research processes, methods and results as well as abstraction and extension should therefore represent basic values of scholarly communication. The possibility to reuse data, materials and results enables researchers and communities to learn from each other and to speed up the production of new knowledge" (Vienna Principles 2016; Accessed July 10, 2020).

\section{There's a badge for that}

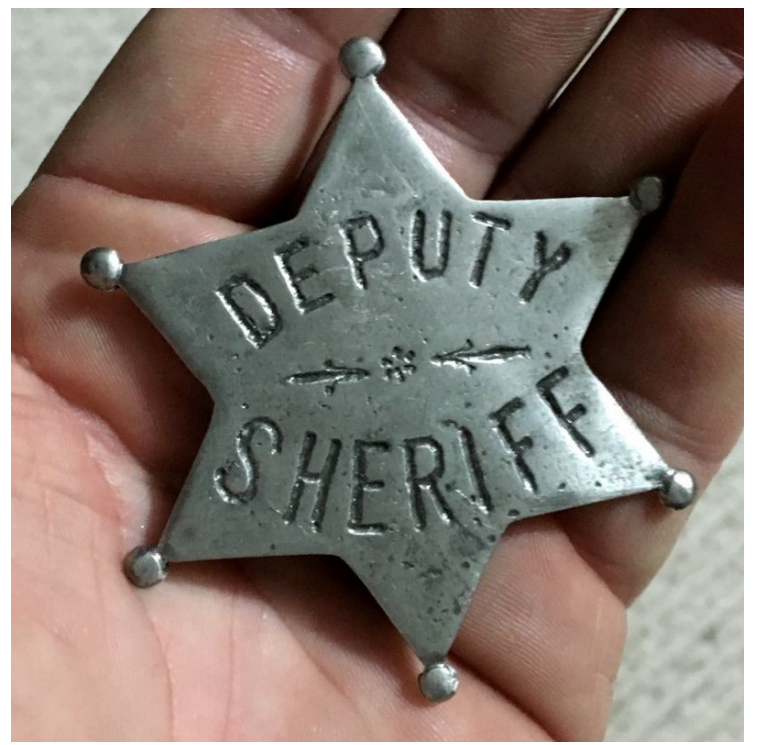

What's wrong with having and celebrating "giants" in your field? We can explore this. Firstly, the goal of exclusive achievement and individual fame requires and produces way too much scarcity in the process. In the game of "giant-making,"recognition points might need to be hoarded, reputation metrics jealously guarded, and ideas (and data) locked away until some strategic moment. Secondly, the practice of acknowledging a science giant requires the production of science dwarves. It's a zero-sum game. If nobody's small, someone can't be giant. Most giants only look large from far away because of the cumulative advantages they were given across their careers. They are standing on the shoulders of privilege. Finally, the desire to be a giant fuels narcissistic behavior, which the academy has an abundance of already.

"As Justice Louis Brandeis, who witnessed our previous Gilded Age, might have said: 'We may have democracy, or we may have praise showered on the heads of a few, but we can't have both"' (Johnson 2019: Accessed July 24, 2020)

In a fiercely-equal, open-science culture, zero-sum games of prizes and awards handed out to would-be giants can be replaced in favor of a larger emphasis on a system of open badges 
that anyone can earn: with intention, time, and effort. The use of badges earned instead of prizes won for recognition of accomplishments would build a reputation economy for the academy that rewards achievement anywhere on the planet, and refocusses attention on science's generative engine: learning and community effort. "Although the edifice of scientific understanding is sometimes envisaged as an accumulation of individual discoveries, in reality science is a community effort comprising innumerable interdependent contributions. Credit is disproportionately awarded to principal investigators for what is truly the product of teamwork, and nearly all scientific contributions are heavily dependent on knowledge obtained earlier... In the spirit of an Amish barn-raising, a celebration of the collective achievement of science should subsume individual achievement" (Casadevall and Fang 2012 [ASM]).

The finite game of "making a name for oneself" in the academy is far too expensive to the academy to allow this to be a central goal of science. Science demands so much already from you: both rigor and wonder, and in generous amounts. "Science is an inherent contradiction-systematic wonder-applied to the natural world" (Lewis et al. 2001).

Because it is important to regularly celebrate open science cultural practices, and contributions to science, and to institutions, and teams, you can create honors that are playful and honest. Science doesn't need fellows in national academies as much as it needs researchers who can get honored for their dedication and their kindness. Be generous to those who are, too. Don't tell your team members to "leave their frowns at home," but hand out medals (perhaps made of chocolate) to those with the most difficulties to overcome, and the best spirit. Give away prizes every week. Cheer when someone earns a difficult badge. Turn learned society elections into lotteries, and celebrate when volunteer leaders chosen at random step up and perform. Find ways to reward as many early career colleagues as possible. In the end, you realize that everyone who makes a serious attempt to do science is already a giant. You didn't notice because you are one too.

You are automatically a member of an elite group: the club of scientists

\section{Exclusivity stops you from diving deep.}

An elite cohort is a group of individuals where some special level of skilling makes these particular individuals eligible to join. An exclusive cohort is a group that has created a scarcity and manages entry. The group reserves the right to join. Private clubs can be really exclusive while not being regarded as elite (at least by others).

Free divers as a cohort can support an elite group among them without needing to consider restricting entry, without becoming exclusive. You want to be an elite free diver? Work at it. Dive deeper. Then dive even deeper. You can achieve elite status on your own. 
The academy is already elite. Only about one percent of humans over the age of twenty-one have a PhD. Only a very small minority of people (with or without PhDs) decide to spend vast amounts of their time exploring unknowns in the universe. This means that the academy has no need to also be exclusive. Like bullshit claims for "excellence" (Moore et al. 2017), claims for exclusivity are counter-

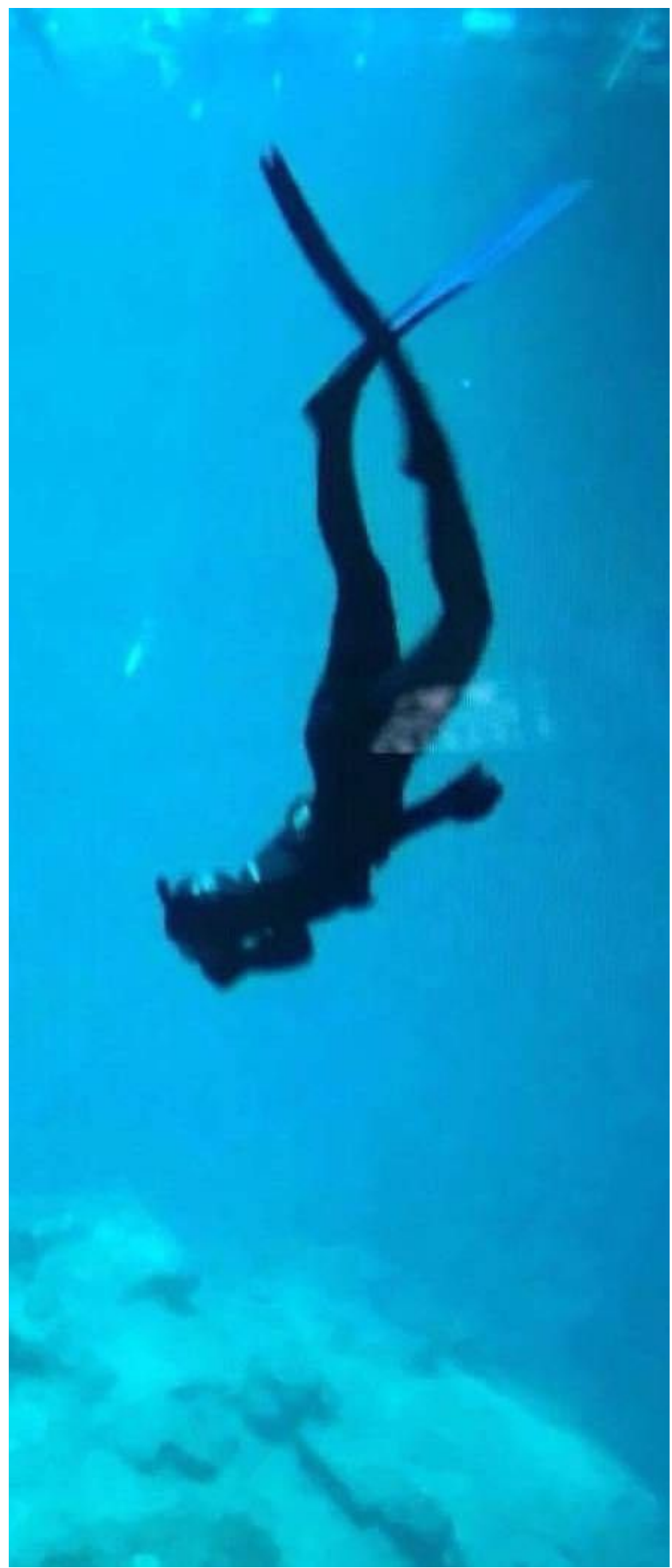
productive. They announce that science can only be accomplished by a selected few. Selected by whom? Editors at Elsevier?

Still, within the cohort of scientists, some are known as really good scientists. These elite scientists are self-selecting. They select how much work they plan to put into doing good science.

"A central claim made by proponents of open and collaborative production is ...based on the argument that commons-based, non-proprietary systems of production are able to draw on a much wider range of human motivations than those deriving from participation in markets, or coercion by managerial command and waged labour. Benkler (2006) suggests that the freedom to co-operate in collaborative ways with others to make things of value to humans, and to be generous and kind (behaviors and patterns familiar to us from social relations generally) motivates people far more effectively and efficiently than traditional market mechanisms or hierarchical models of social organisation" (van Zwanenberg et al. 2017).

\section{Open science is an escalator to becoming elite.}

Open science will help you to do what you 
need to do to become an elite scientist: build your knowledge, your craft, and your reach. Share your research work flow so others can offer advice and kudos. Share because sharing accelerates the feedback that drives new ideas in your own work. Share because others will take your data to places you never considered.

Open science is the smart way to become elite. Be elite not through some erzatz "journal impact factor" but by sharing your work openly, and by being generous with your colleagues, particularly those few who are struggling with the same object of study you have chosen.

You and a handful of other scientists have somehow been drawn to the same problem, the same phenomenon. Together you can dive deeper into this problem than you can ever go alone. The only impact factor you need is the one that comes in your inbox from a colleague thanking you for solving one of their research pain points.

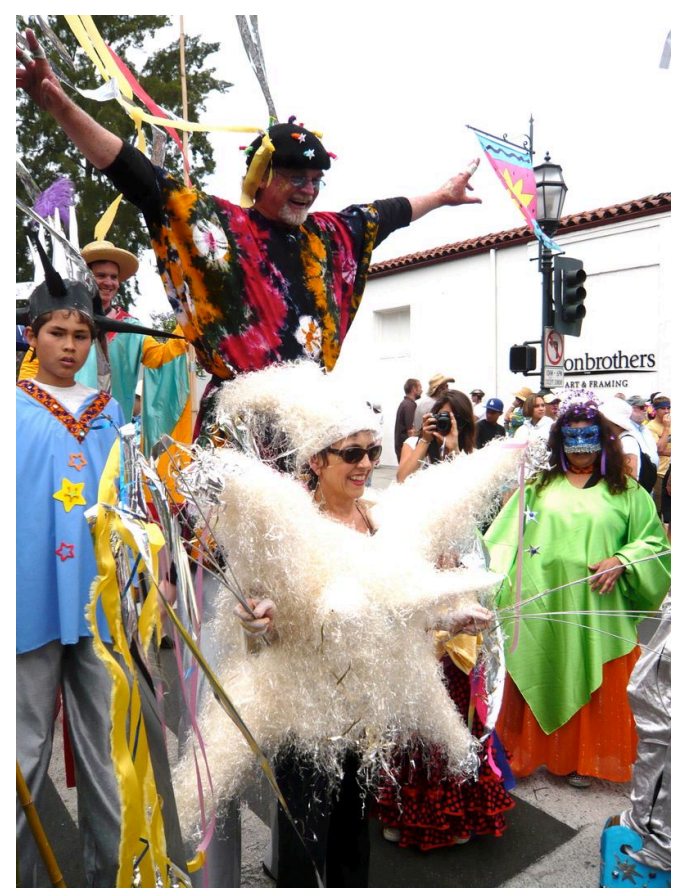

Marc McGinnes, who taught for decades at UC Santa Barbara, puts on stilts every year to become an "occasional giant."

Afterthoughts: If you still want to be a giant, be a giant for your family, be a giant in your town.

Perhaps there used to be giants, back when the only way to fund science was to attract the attention and the purse of a king. If the person paying your rent is named de'Medici, perhaps you should get used to wearing stilts, just ask Galileo Galilei. The main lesson of that famous "standing on the sholders of giants" quote is that if you are going to be a life-long jerk, pop some really nice sentiments on your blog that people might remember you by three hundred years later. Even then, someone will write a book about what an asshole you were. 


\section{Demand Sharing: a Real Sharing Economy for the Academy}

In a famous letter of 1813, Thomas Jefferson compared the spread of ideas to the way people light one candle from another:

"He who receives an idea from me, receives instruction himself without lessening mine; as he who lites his taper at mine, receives light without darkening me."

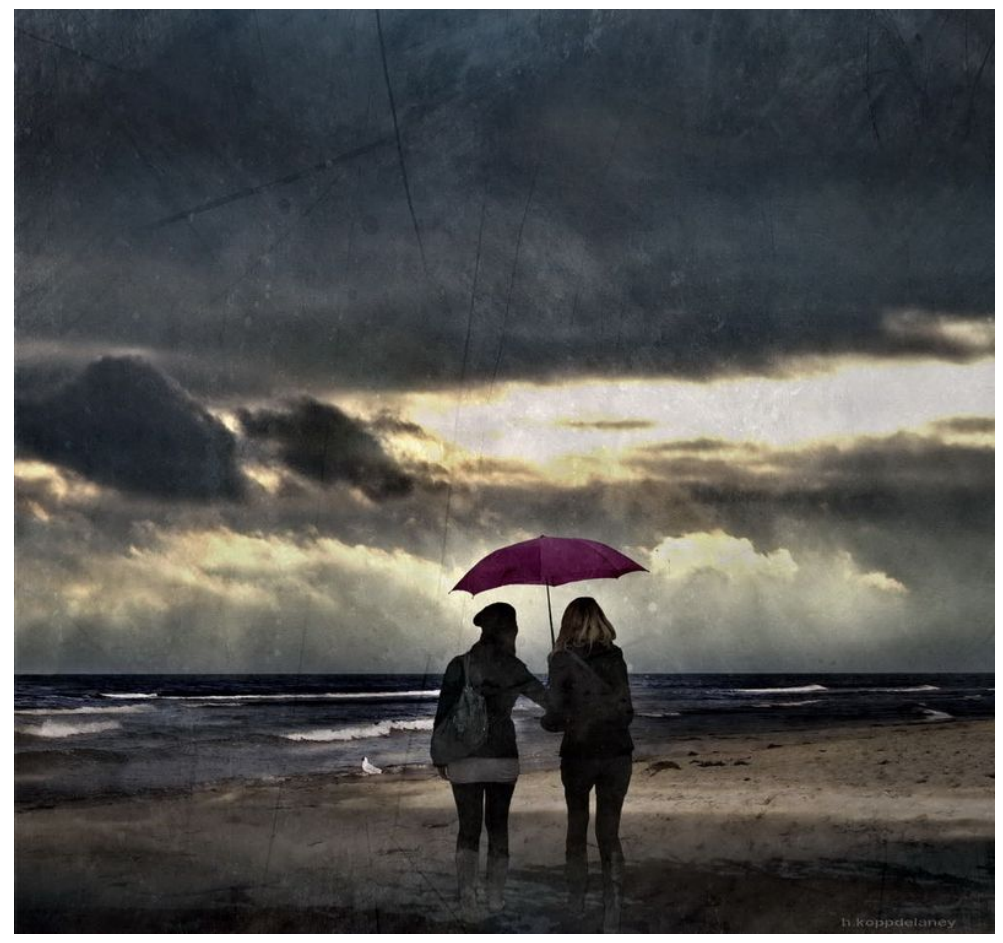

Photo Credit: Hartwig HDK on Flickr, CC By-ND 2.0

Demand sharing means you can ask for everything you need to do your science... with one proviso...

We've all heard about the "sharing economy," where we can gain new streams of income or convenience by simply sharing excess capacity (that spare room, the car ride, an electric scooter, etc.). And we've been told since childhood that sharing things we no longer need can help those with greater needs. Most of us feel we have a good idea about what it means to "share." But then most of us are also mistaken, and here's why.

Anthropologists who look at the ethnographies (and who do their own) of hunter-gatherer groups, and who sometimes also look at modern attempts to create sharing economies (e.g., Uber, Airbnb, etc.), tell us several things about sharing that most of us may find new and different from what we expected (Widlok 2016; Suzman 2018; Accessed May 6, 2019). 
These ideas about sharing, synthesized from the study of human groups that have been successfully building their own lives for tens of thousands of years, say to us that we have "sharing" almost completely wrong.

For example:

- Real sharing is not charity. Charity is an artifact of the marketplace (and of personal wealth) and the logics of artificial scarcity.

- Sharing something you own that you are not using (like a spare room or space in your car) in exchange for cash is just another form of market transaction.

- Giving away things that you don't need or no longer want is not a good example of sharing. This is an edge case.

\section{Demand Sharing: share what is most important to you. Get what you need in return}

In this Handbook, we use the phrase "demand sharing" to designate a culturally advanced form of sharing, a type of cultural behavior that has been in widespread use of the majority of the human population for tens of thousands of years, and only recently eclipsed by marketplace logics in the past two to three hundred years. "Millions of years of evolution have designed us to live and think as community members. Within a mere two centuries we have become alienated individuals. Nothing testifies better to the awesome power of culture" (Harari 2014). Additional information about demand sharing is available elsewhere in the Handbook.

\section{Society uses demand sharing to fund its needs}

A rather good (perhaps unexpected) example of demand sharing in modern society is having your representative government demand a tax that everybody pays, which then, for example, supports your state's public colleges and universities (and pays your salary). That's right; taxation is how a society demands of itself those resources it needs to prosper (Widlok 2016).

Another example is sharing within a household, where family members can grab a snack from the refrigerator without much bother or need to justify or account for their choices. In the case of the academy, the "refrigerator" is the rapidly expanding corpus of digital research objects, and the family is fellow scientists who stock this with the outputs of their work, and who can then dive in and grab what they need for their own research. Note: this is a never-empty refrigerator, as these digital objects are not used up by their taking. Note again: they are anti-rivalrous: they gain value when they are shared. This is something every open scientist needs to remember. 
"[L]earning is taken as much as given" (Godin 2019; Accessed May 6, 2019).

\section{Learning is demand sharing for knowing}

Teaching and learning already require demand sharing. As an open scientist you've probably taught in a variety of classroom situations. Your students asked questions to extend their learning. Your best students (bless them) outright demanded to be taught. They marched into libraries (buildings, or on-line) and demanded the resources they need. They came to your office hours and demanded answers to their quandaries.

This means that nearly every scientist is well versed on how to participate in a demandsharing economy. First, the state demands that its citizens fund the university, supporting teachers and learning. Then the student shows up and demands to be taught. We all did this as students. It's not obscure, it's how we learn.

Imagine a professor giving a lecture who stops in the middle and says, "This next part is really interesting; if you want to learn it, go to my app on your phone and deposit $\$ 10$." This should sound bizarre to you: if it doesn't, then the neo-liberal university is your real home. In part it sounds strange because the professor's salary is already paid, hopefully through taxes. But mainly, it sounds wrong, as professors (who were once students) are completely happy for their students to learn. These learning moments in the classroom are seen as socially important and personally rewarding. When a student asks you a question, you do your best to help them learn something new. Note: making your students buy a textbook you authored, for a price that might equal the rent they pay in a month, should also sound strange.

In a hunter-gatherer culture, such as that of the Ju/'hoansi, when a child comes to your fire and demands a bit of meat from your catch, you give it to them. Like food at a huntergatherer fire, information in a university is something that can be demanded. Demand sharing in education is a type of cultural economy where the norms and rules-the times and places, the manner of asking, the desire to teach and the value of learning - are wellknown, without being written down. Students know they cannot demand the answers to a quiz in advance. What is sometimes forgotten is the need for and role of kindness in these interactions.

\section{Got a PhD? You know how to demand what you need}

This means that you already know how to do demand sharing. Let's look how demand sharing differs with what we just described as poor examples of "sharing."

- You don't give your classes as a form of charity (even though you may consider your own salary inadequate). You are a professional. Teaching is important. Your students 
have legitimate demands on your knowledge and your kindness. Passing on knowledge is why you teach.

- You also don't teach your students content that you find worthless to you or loan them books that you are no longer satisfied with, unless these books are instructive in other ways. You share what is really important to your professional life: the best knowledge you've acquired.

- You expect students (at least, grad students) to demand from you what they need to learn and grow as scientists.

\section{Demand sharing means sharing what is valuable and important to your research}

This is the proviso we mentioned above. The same demand-sharing logic that collects the taxes that pay your salary, and enables your students to learn, also enables the academy to manage its knowledge resources for the benefit of all scientists, and the planet through the internet. Until today, a scientist might legitimately point out the huge amount of processfriction that would overly complicate sharing her data or workflows. A lot of the work of open-science advocates in the last two decades has been focused on reducing that friction through web-based platforms and services. Much of the remaining friction is cultural; linked back to institutional practices that do not reward or actively punish open resource sharing.

In an open-science, demand-sharing academic culture, sharing as much of your research as early as possible is a virtue strong enough to be a norm. Share what matters most to you: your methods, your findings (even null fundings), and your data. Share your ideas openly too, not simply those ideas that you have no interest in pursuing and every interest in having someone else pursue. Share your knowing by listening and adding to the conversation.

Open science requires generosity with a simple promise: each scientist will get more than they give. That's the bargain the academy makes with you when you join and actively participate in the open-science academic society; a bargain that today gets broken all too often. It is the promise of the trove of knowledge that the academy maintains in libraries and repositories. This bargain is bolstered by the network effects of academy organizations. Demand sharing optimizes this bargain across academic networks and clubs (Redaction 2016; Accessed June 1, 2019).

Sharing imbeds your work into the community of science as a gift, a form of offering that also signals your membership. Sharing includes reviewing and acknowledging the work of your peers (See also: Perils of peer review). The open-science community creates its internal authority through relentless self-critique. 
This authority works through a special soft of reciprocity and a level field of mutual status. As Polanyi (1962) noted, "[0]nce the novice has reached the grade of an independent scientist, there is no longer any superior above him. His submission to scientific opinion is entailed now in his joining a chain of mutual appreciations, within which he is called upon to bear his equal share of responsibility for the authority to which he submits." This reciprocal authority of "mutual appreciations", based on openly shared and critiqued knowledge is the basis for all applications of authority and leadership in an open-science academy.

The offerings you provide to the "republic of science" (Polanyi 1962) lend you the cultural capital to demand the resources you need for your work from the abundance of openaccess resources, and the knowing of others in your field. These, in turn, offer up their research for your use. As Hyde (2009) notes, the "constant and long-term exchanges between many people may have no ultimate 'economic' benefit, but through them society emerges where there was none before"; your contributions help create the academy society.

Amplified by the internet's global reach, these exchanges expand and accelerate the process of science. You share the most important ideas you have, even at the risk of being scooped, because getting the most important work done now-whether you do this or someone else does (and attributes you with the idea) - moves science forward. You share your research results, all of them, knowing you will be critiqued by your peers, as you will also critique theirs.

One more advantage of demand sharing is the occasion where you find a piece of information outside your close associations and intellectual domain, and you demand (ask) to gain more knowledge so that you can include this in your work. Demand sharing activates weak ties (Granovetter 1983), bridging between knowledge domains to spark innovation. "From the perspective of innovation, it's even more important that the information arriving from one of those weak ties is coming from a different context" (Johnson 2011). Of course, you will also, on occasion, be asked to give out knowledge around your research, opening up weak ties with new potential collaborators.

"The self-image of humans who are embedded in sharing relations is not one of homo faber who creates his or her world out of nothing and without anyone else. Rather it is an image of what I have called homo sumens ... who takes into use what is available through the company of others and that can be claimed from them" (Widlok 2016).

\section{Academic clubs: collectives for research collaboration}

Demand sharing is a dense cultural practice, with its own behavioral expectations. When you share, you signal your desire to be included in the community. What you must learn, 
then, are the guidelines for demanding resources. "[T] he problem is not one of deciding what to give to whom but rather what to demand of whom. The onus is on the potential receiver to make his or her claim acceptable and the rules for appropriateness are not about acceptable giving but acceptable demanding" (Widlok, ibid; emphasis added).

The cultural shift to demand sharing will create a social basis for new science collectives, for "clubs" that share internally as though the club were a single, social organism. These formations are not entirely new. R\&D Think-tanks have been funded for this purpose, and the NSF in the US spends a billion dollars a year funding academic workshops to assemble temporary collectives to solve common problems. "Club goods" are non-rivalrous inside the club, but not necessarily without shared costs (Hartley, et al. 2019). Science club goods have the additional property of being anti-rivalrous. Thomas and Brown (2011) describe these clubs as collectives, "Collectives are made up of people who generally share values and beliefs about the world and their place in it, who value participation over belonging, and who engage in a set of shared practices. Thus collectives are plural and multiple. They also both form and disappear regularly around different ideas, events, or moments." Collectives enable collaboration across the internet, inform team-building and open up the situations for shared knowing.

The cultural practices of demand sharing will be emergent in the academy as open resources-including access to and discoverability of collaborators-become increasingly available in the next decade. This Handbook will help you to kickstart your own collectives, and forge demand-sharing cultural norms that suit your situation; see also Building new collectives.

"That ideas should freely spread from one to another over the globe, for the moral and mutual instruction of man, and improvement of his condition, seems to have been peculiarly and benevolently designed by nature, when she made them, like fire, expansible over all space, without lessening their density in any point, and like the air in which we breathe, move, and have our physical being, incapable of confinement or exclusive appropriation. Inventions then cannot, in nature, be a subject of property" Thomas Jefferson 1813 letter, quoted in (Boyle 2008).

Together with "fierce equality," demand sharing as a cultural norm can help realize an actual sharing economy for the academy, separated from the arbitrary scarcity of the neoliberal marketplace; a special type of gift economy grounded in mutual appreciation, rather than reciprocity. The demand sharing economy helps scientists grow networks that are far larger than those available through simple reciprocity (Grant 2013), and also far more emotionally satisfying (no need to keep track of each transaction). The particular practices of demand sharing will need to grow inside thousands of institutions across the globe. 
A goal of this Handbook is to give you the resources you need to build demand-sharing logics inside your academy homes. You can be a demand-sharing change agent by sharing your research objects and your research questions and problems; by listening more and adding your knowledge when asked. Demand answers from others; learn together. It's science, not alchemy. You are not alone. 


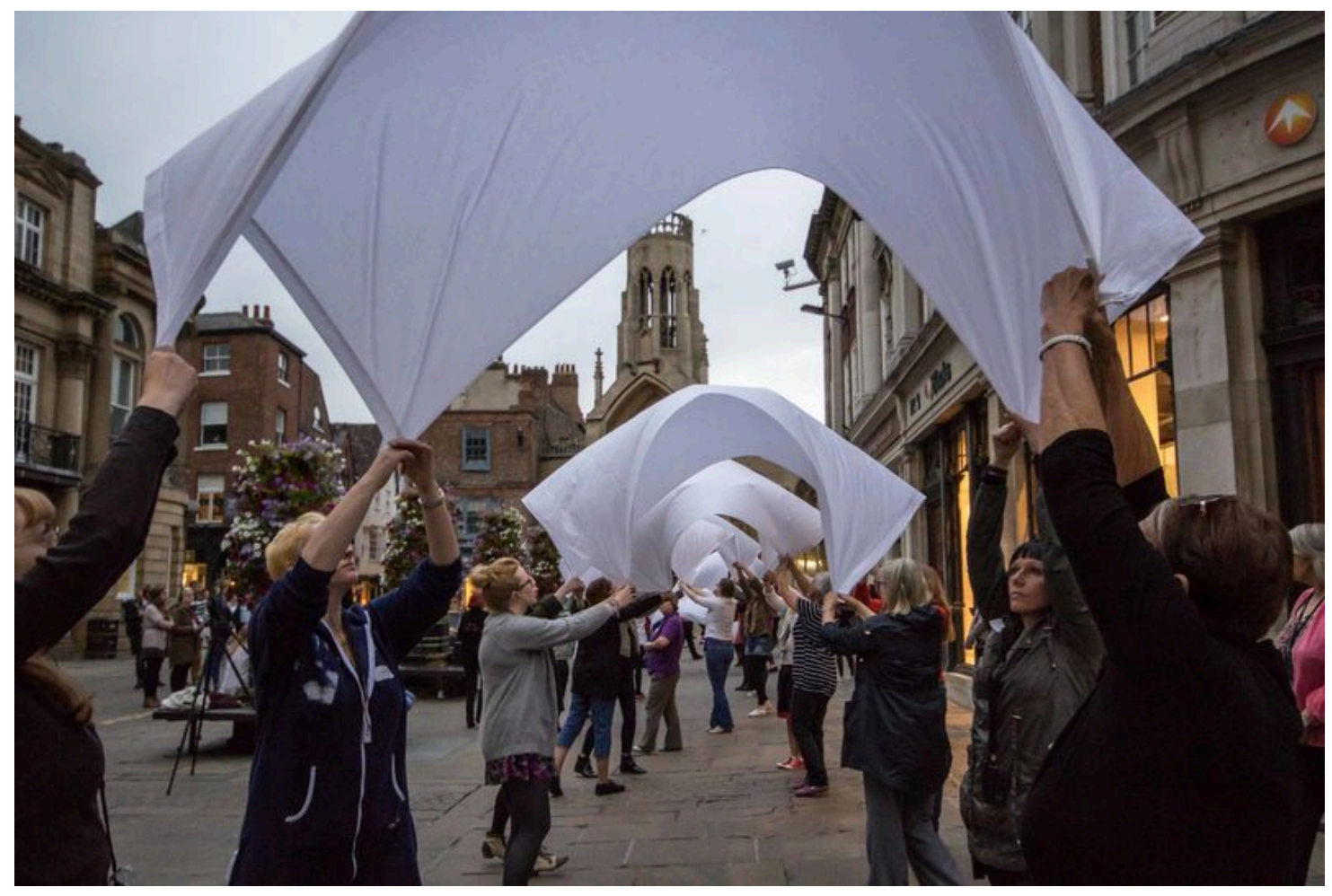

Culture gives your gift the meanings it needs

\section{Building a gift economy: the dance of open science culture}

"I am not saying science is a community that treats ideas as contributions; I am saying it becomes one to the degree that ideas move as gifts" (Hyde 2009).

"The specificity of [demand] sharing... is rather that it also constitutes sharing in, granting access to the flows of objects, their intrinsic goods, and their intrinsic value" (Widlok 2013)."

"That is the fundamental nature of gifts: they move, and their value increases with their passage. The fields made a gift of berries to us and we made a gift of them to our father. The more something is shared, the greater its value becomes. This is hard to grasp for societies steeped in notions of private property, where others are, by definition, excluded from sharing" (Kimmerer 2013).

So, you will want to change the culture of your organization to enable "demand sharing" (See: Demand Sharing). This is something the Handbook encourages, and, after reading this section, you will understand why. The goal of this culture change is to build an internal 
economy for the scholarly resources you are now assembling. This economy will do two somewhat intertwined things: optimize the value of these resources and support their use through practices that respect and enable fierce equality across the global "republic of science" and beyond.

The argument here is that the current, scarcity-based market economy that has penetrated inside the academy does neither of these things well enough, and sometimes not at all. Instead, it promotes hyper-rivalrous games to capture funding and prestige, while it marginalizes and silences the long-tail of science talent across the globe. What the market economy does do really well is capture external motivations that appear to power efficient use. Instead, these motivations infest the academy with unavoidable conflicts of interest and perverse incentives.

This form of economy ends up externalizing much of the value of research goods. These become properties held outside of the academy_from which they were born; this happens today, every time you gift Elsevier or Wiley the copyright to your research article. While this arrangement frees the academy from investing in the repositories that could hold these goods internally, and in recognition schemes to highlight great work and show gratitude to science teams doing this work, the cost is significant and ongoing: the academy needs to pay over and over again to access its own resources.

\section{Slow is smooth and smooth is fast: with enough culture you can go slow enough to accelerate science}

"What peer producers are doing-for now mostly in the sphere of 'immaterial' production of knowledge, software, and design -is to create an abundance of easily reproduced information and actionable knowledge; that which cannot be directly translated into market value, because it is not at all scarce-on the contrary-it is over-abundant" (Bauwens 2012; Accessed 10/2018) .

The cultural practices that support demand sharing are not simple at all. Not nearly as simple as the market transactions we do every day. They require active, culturally coded, shared intentions. Think of them as a learned, shared cultural "choreography." Every member of the group knows how to dance with the group. These practices are durable enough to have sustained hunter-gatherer groups across the planet for tens of thousands of years, sophisticated enough to enable entire small societies to manage almost all of their internal transactions, and logical and transparent enough so that children do learn and follow them. Peer-to-peer production uses these same cultural practices to build the infrastructure of the internet. 


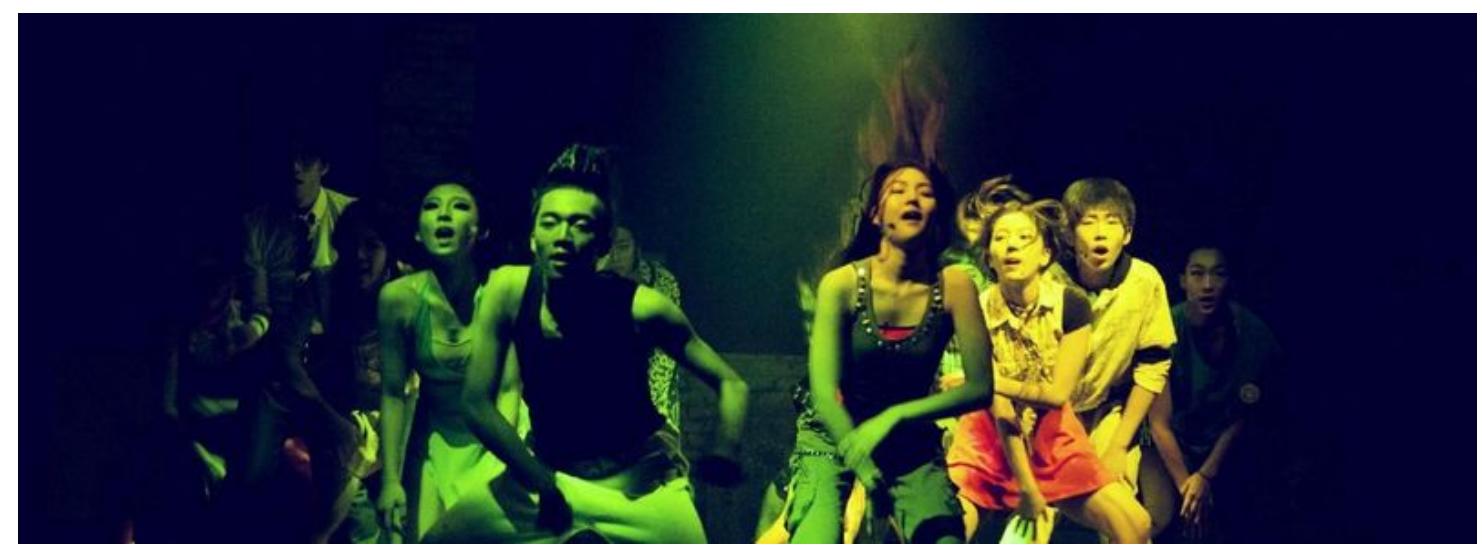

"Almost everyone [in the social sciences] continues to assume that in its fundamental nature, social life is based on the principle of reciprocity, and therefore that all human interaction can best be understood as a kind of exchange...

...Exchange is all about equivalence. It's a back-and-forth process involving two sides in which each side gives as good as it gets" (Graeber 2011).

\section{Reciprocity and gift economies unpacked}

Let's look closer at socio/anthropological notions of a "gift economy" and "reciprocity," and more recent work in economic history and economic anthropology for new insights to the "sharing economy." In anthropology, reciprocity is a topic that has launched a thousand dissertations, that has informed entire schools of theory and argument, and that has been the wellspring of anthropology's connection to economics since Marcel Mauss's book, The Gift, was published in French in 1925.

The Handbook assumes your goal here, or one of them, is to avoid needing to become an anthropologist in order to be a culture-change agent. Here are the basics of gift economies and reciprocity you might call upon without further study. Let's start with the conclusion: demand-sharing is a form of reciprocity that requires active, intentional cultural practices to deliver an optimal return for the academy. Demand sharing is a practical/theoretical upgrade on the notion of the academy as a "gift economy." It describes a relationship in practice between scientists and science, between scholars and the academy.

\section{Start with reciprocity}

At its core this is a durable obligation to interact with others. So, this behavior is culturally coded. Inside the community, exchanges get made that motivate future exchanges. Importantly, these obligations are never designed to achieve a final closure. Reciprocity in life and in the academy is a feature of infinite play. Reciprocity colonizes your future by 
enrolling you in longitudinal practices of giving and getting. When your child finishes college, you do not present them with a bill for all of the expenses they cost you growing up. If you do, you are planning to never see them again.

Market transactions (and also theft) avoid this type of ongoing obligation. So does charity, mostly. When you sell your old car to a stranger for cash, you have every hope that you will never see them again. When a thief breaks into your office and takes a computer, you do not expect them to give you something back in the future. When you give some food to a homeless person on the street, you don't expect to get something material back from them later on.

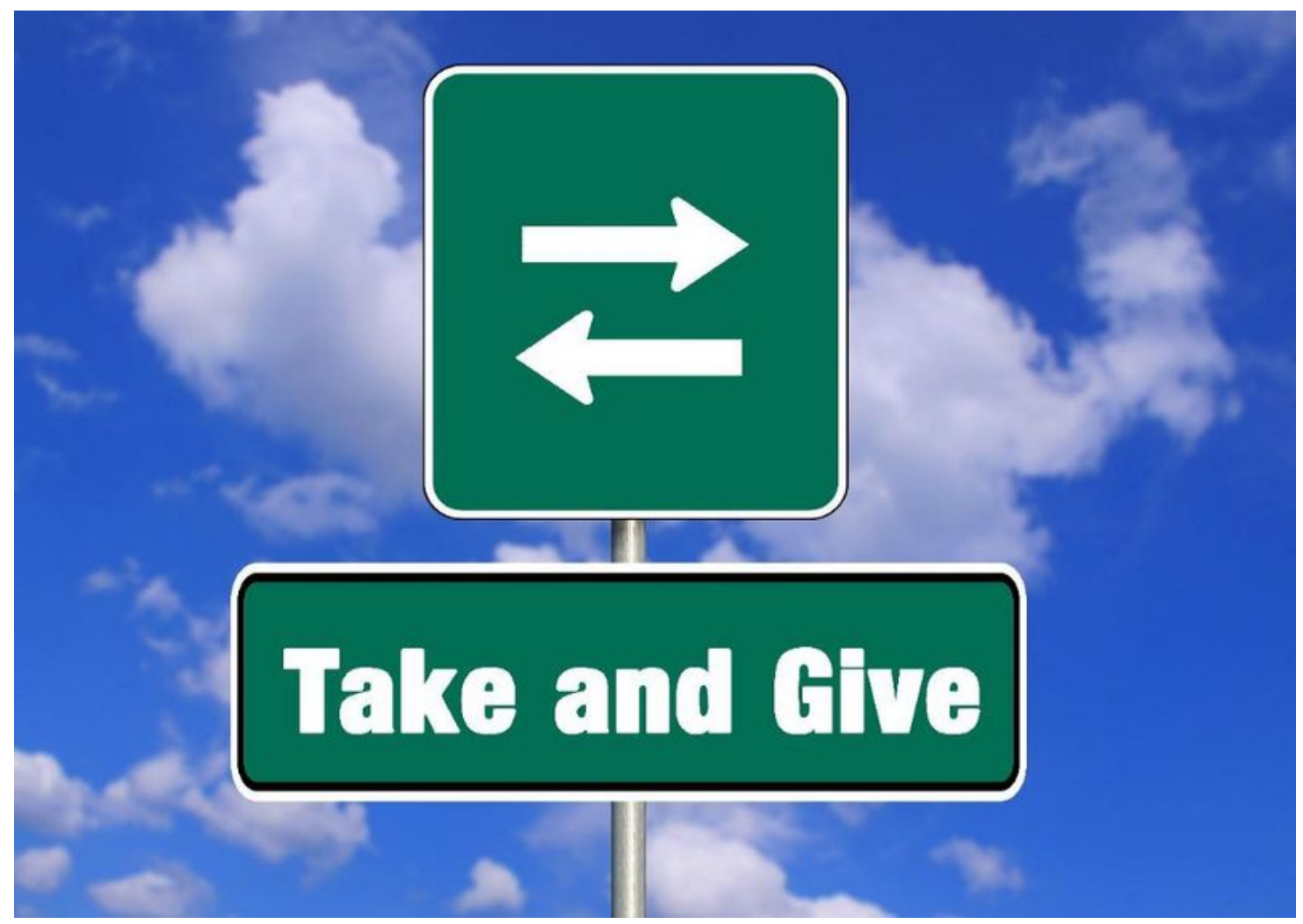

Reciprocity takes more work (cultural and emotional) to maintain than direct market exchanges. It takes a shared understanding of the implied obligations for reciprocity to succeed as an economic logic. For this reason, reciprocity works best inside a community. Transactions between different communities involve more rule-making, and less cultural coding. Considering the academy as a community, or a collection of like-minded communities, some form of reciprocity is an economic logic that fits very well, once the cultural practices for this become normative. 
In some cases, the implied obligations of reciprocity can negatively color the relationships between individuals: "When favors come with strings attached or implied, the interaction can leave a bad taste, feeling more like a transaction than part of a meaningful relationship. Do you really care about helping me, or are you just trying to create quid pro quo so that you can ask for a favor?" (Grant 2013).

Reciprocity can be generalized or more specific, and both forms can be active in the same culture. "Generalised reciprocity is characterised by Marshall Sahlins as being marked by a weak obligation to reciprocate and an indifference to the time, quality or quantity of the return. It is typically the behaviour found between such closely related people as parents and children or siblings, where asking for things is widely acceptable..." (Peterson 1993). Generalized reciprocity brings in a key feature of demand-sharing: the right to ask for what you need. You can say that demand-sharing is a certain specific type of generalized reciprocity, highly coded to be efficient and sufficient across an entire group (not just a family). So, what about gift economies?

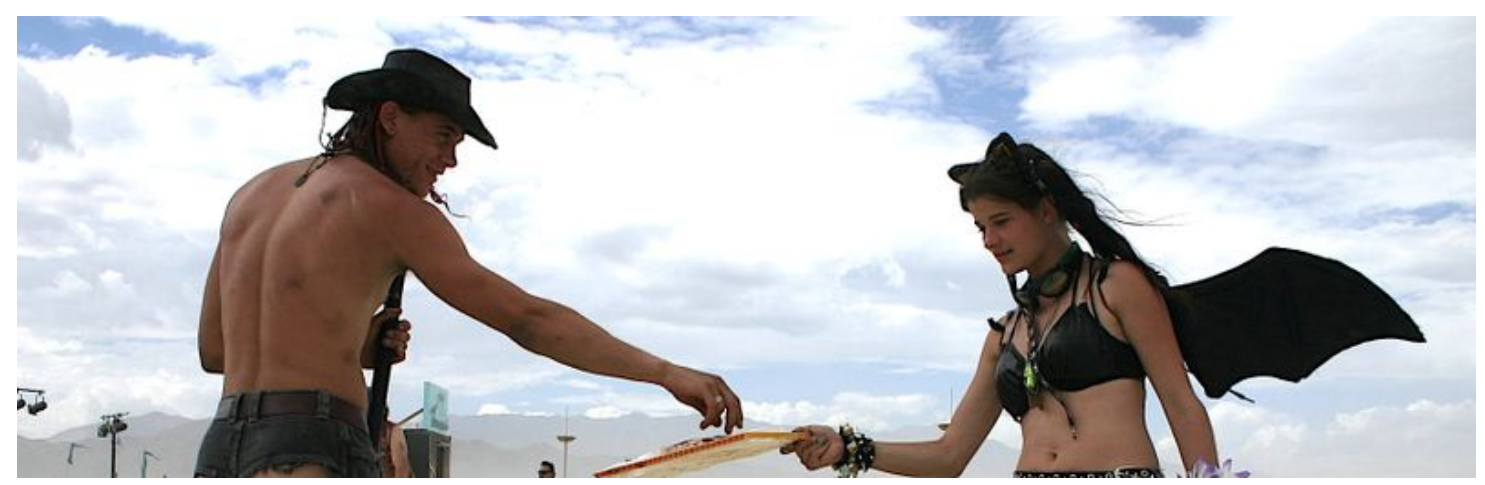

Gifting on the Playa. Photo Credit: Bill Dimmick on Flickr CC

"Gifts in Burning Man culture are offered unconditionally. In the case of individuals who contribute to our community, such gifts are relatively easy to accept, and it is only common courtesy to recognize these givers and their contributions. ...This is an application of the Principle of Radical Inclusion" (Harvey; Accessed June 17, 2020).

"These remarks on the scientific community are intended finally to illustrate the general point that a circulation of gifts can produce and maintain a coherent community, or, inversely, that the conversion of gifts to commodities can fragment or destroy such a group. To convert an idea into a commodity means, broadly speaking, to establish a boundary of some sort so that the idea cannot move from person to person without a toll or fee. Its benefit or usefulness must then be reckoned and paid for before it is allowed to cross the boundary" (Hyde 2009). 


\begin{abstract}
“The important lesson I learned from Adam [Rifkin, a Silicon Valley entrepreneur] is that you can be a genuinely kind-hearted person and still get ahead in the world' [quoting Stephanie, a LinkedIn recruiter]. Every time Rifkin generously shares his expertise or connections, he's investing in encouraging the people in his network to act like givers. When Rifkin does ask people for help, he's usually asking for assistance in helping someone else. This increases the odds that the people in his vast network will seek to add value rather than trade value, opening the door for him and others to gain benefits from people they've never helped-or even met. By creating a norm of adding value, Rifkin transforms giving from a zero-sum loss to a win-win gain" (Grant 2013).
\end{abstract}

\title{
Understanding gift economies
}

Gift economies span from indigenous peoples to science cohorts, with Burning Man in the mix, somewhere. Speaking of indigenous gifting, Kimmerer notes; "The essence of the gift is that it creates a set of relationships. The currency of a gift economy is, at its root, reciprocity. In Western thinking, private land is understood to be a 'bundle of rights,' whereas in a gift economy property has a 'bundle of responsibilities' attached" (Kimmerer 2013). A great way to dive into the academy gift economy is to read Lewis Hyde's (2009) book, The gift: Creativity and the artist in the modern world. In his book, Give and Take, Grant (2013) explores giving as a socially valuable practice for $21^{\text {st }}$ Century commerce. Both Hyde and Grant use gifting to illustrate the value of "openness." For Hyde, openness produces scholarly objects that grow in value as they are shared without regard to direct compensation. For Grant, openness creates weak ties across vast networks where generosity is also generative for creativity and innovation.

A gift economy uses gifting as its primary, and/or its celebrated form of exchange. There are no purely gift economies; people create exchanges for complex reasons that might not fit in this description, even when they use gifting for most exchanges (Graeber 2001). At Burning Man, where you can find someone to gift you any recreational drug you might desire, you can always purchase coffee at Center Camp. Gift economies co-exist with other forms, such as market economies. Hyde (2009) calls this a "mixed" economy.

In a non-gift economy, gifts can still be reciprocal, even if the return gift is only an expected "thank you." Families may send out holiday cards and keep careful track of the cards they receive in return. They trim their card list accordingly. Birthday gifts or dinner invitations to friends open up expectations of similar goods coming back. Edge cases are also available. Oprah Winfrey added to her fame by giving away cars (accessed 06/20/2020) on her television show. Philanthropy channels donations to a range of causes where the return is not a gift, but some resolution of a deficit or a wrong. In what way is gifting essential to the academy? 
The idea here is simply to catch the central meaning of what a "gift" is within a scholarly community. Hyde points back to Warren Hagstrom's work on The Scientific Community (1965): "Hagstrom writes that 'in science, the acceptance by scientific journals of contributed manuscripts establishes the donor's status as a scientist-indeed, status as a scientist can be achieved only by such gift-giving - and it assures him of prestige within the scientific community"' (Hyde 2009). However, this exchange of status for the gift of a research article was, and never is that simple. The bundle of responsibilities in this exchange includes (not exclusively) assurances of research integrity, access to data (optimally), and continuing conversations about the research results. The community gets to demand what it needs to realize the value of this gift for science.

\section{Demand sharing is different}

Demand sharing is focused on a relationship between individuals and the group. Whereas a gift economy can be focused on how particular transactions are handled between individuals across their lifetimes, demand sharing is grounded on belonging to a group and knowing when and how to offer and to ask for goods from the group. You could say this is a certain form of gift economy, with added group-sanctioned cultural practices. Social distancing during a pandemic is a type of demand sharing. In this case, the group benefits when each individual participates, and the individual benefits when the whole group acts in concert.

Demand sharing resembles "tolerated scrounging" (Widlok 2013). There is no score to keep, as in monitored reciprocity, and no specific value attached to the goods, as in a market. In place of these are cultural practices and norms that work to preserve the process of sharing, guarantee sharing to all individual members, and protect the integrity of the shared resource pool through active governance. This is precisely what "commoning" in the academy supports. 


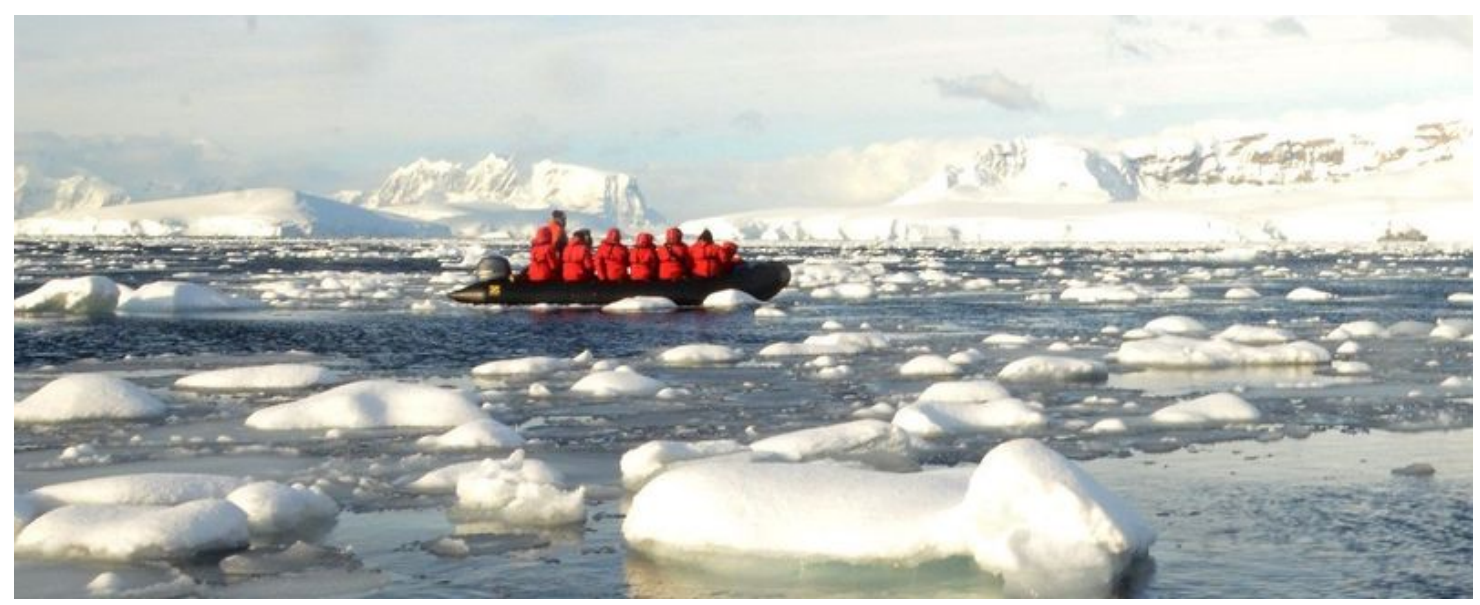

On the open science expedition, nobody gets left behind

\section{Demand sharing is the economy for the commons}

Demand sharing practices require active cultural intention to remain clear and durable. You cannot put demand sharing on autopilot. Rules are less useful here than strategies (See: Making statements about open science). One non-hunter-gatherer example of demand sharing is what is called "expedition behavior." "Expedition behavior involves putting the group's goals and mission first, and showing the same amount of concern for others as you do for yourself. Jeff Ashby, a NASA space shuttle commander who has flown more than four hundred orbits around Earth, says that 'expedition behavior - being selfless, generous, and putting the team ahead of yourself-is what helps us succeed in space more than anything else."' (Grant 2011). Expedition behavior also demands that the group leave no expedition member behind.

Demand sharing begins with a recognition of the legitimate demands from others. It is other-focused. Instead of serial, disengaged market transactions that have no consequent advantage for the group, demand sharing requires and rewards engagement with others. Consideration of others, and consequent consideration by others, creates social closeness: it is a holding close of others into a sharing society.

Demand sharing has been "operationalized" in smaller societies for millennia. Demand sharing practices are local and as complex as their locale requires. "...sharing is in itself a complex phenomenon, more complex than usually imagined by those who are not participating in the economy of sharing on a daily basis" (Widlok 2016). Responding to the specific demands of research arenas, science also groups its activities into smaller societies of researchers that share their own province of infinite play-their own precinct of phenomena, theories, and methods. Within and among these groups, demand sharing 
practices will become as complex as required to fulfill the needs of the group to discover, access, and mine their shared resources.

"A basic goal of provisioning is to reintegrate economic behaviors with the rest of one's life, including social well-being, ecological relationships, and ethical concerns" (Helfrich and Bollier 2019).

\section{Investing in, provisioning from, and sustaining scholarly commons for infinite play}

The main kind of "demands" in a demand-sharing academy are demands that new research be shared with colleagues in a manner that promotes rapid reuse and further knowledge generation. You can consider these as "investment demands." These demands provide a valuable return for each individual scholar and team, which only need to add their work into a shared repository in order to get culturally-supported access to the entire corpus. You add your "carrot" (or onion, or whatever) to the shared research soup bowl, and you get a whole meal back. Only this bowl is never empty, as its ingredients are digital and antirivalrous. So, you get a shared abundance of meals. These are "provisioning demands." You get your fill of the latest data and research findings from across the planet.

A second group of demands center around science play (See: Open Science and infinite play). Within a culture of demand sharing, scientists and teams can demand that their institutions support science across its horizons, and through time within and beyond the lives of individual scientists. This means reaffirming those freedoms that allow science to advance at its own pace and without external influence, and provisioning science teaching and research as a public good (Newfield 2016). These new demands might include a durable, guaranteed minimum income for all researchers, say, and more university funds for new projects and science infrastructure. Freed from the enormous friction of selfinterested finite games where stealing ideas and the fear of "getting scooped" guide a lack of sharing (Hyde 2009), the academy can focus on stewarding its resources and mining new knowledge from these.

The third group of demands are governance and stewardship responsibilities and activities that require individuals as commoners to work to maintain pooled resources and effective governance strategies across time. This governance "overhead" is inherently problematic when the pooled resources are open to all to use. One solution to this problem is to localize the resources (Neylon 2017) and task those who use them a lot to step up and be more active in their stewardship. This is one functional reason why scholarly commons (plural) will need to be localized for governance, and globalized for impact. The immediate issue this solution creates is the need for robust interoperability among the commons, including some sharing of cultural norms for mutual use of combined resources. 
The last demands are really the first ones in the careers of scientists: the demands that students make on their teachers and schools to provision their learning path. If "tolerated scrounging" describes how scientists gather their research goods, teaching the next generation of scroungers means more than opening up scientific content access and understanding. Open-science teaching includes inculcating cultural knowhow about the norms and practices of commoning in scholarly commons. 


\section{As an open scientist, you're good-to-get what you need}

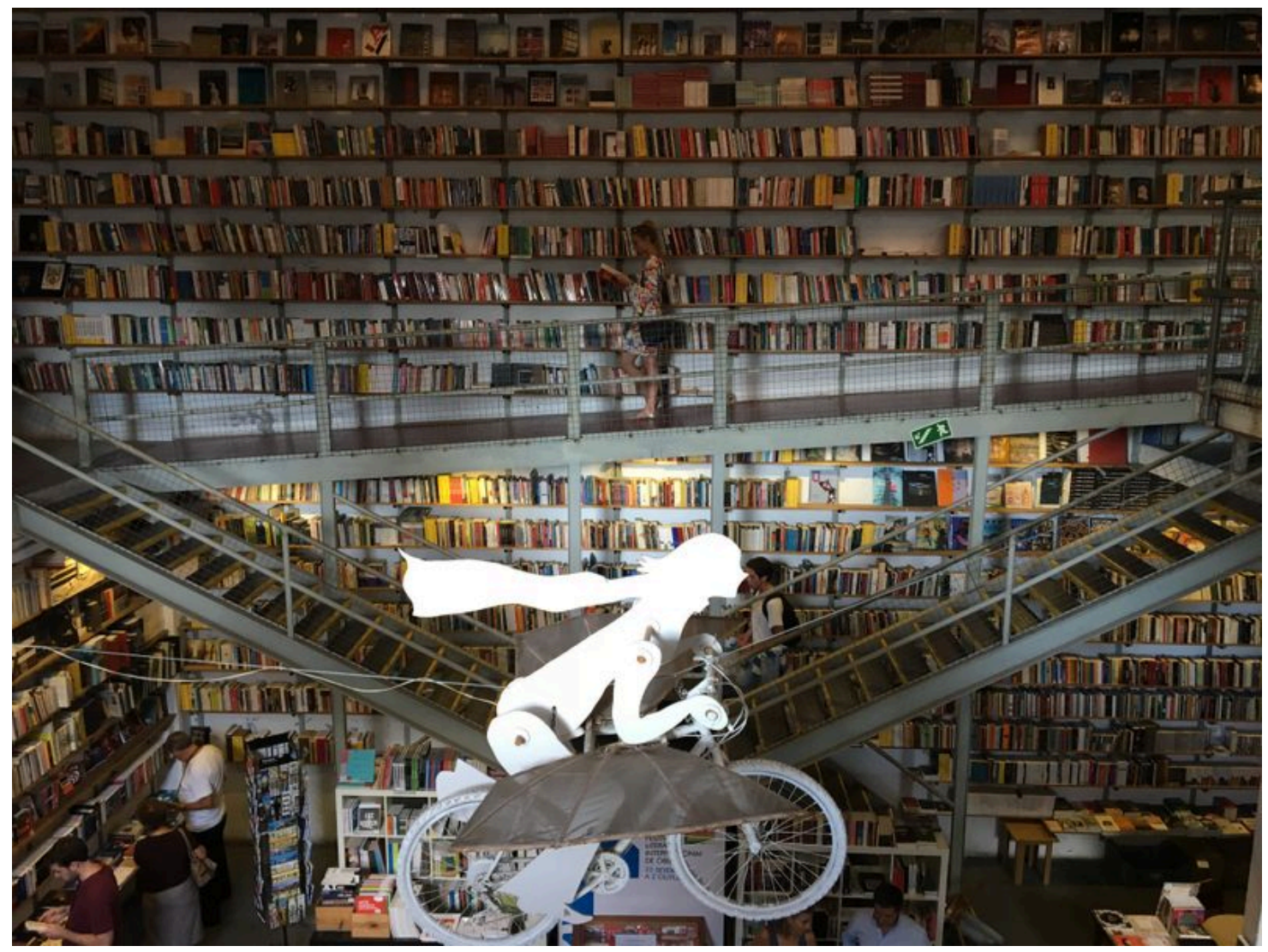

You're a scientist, with a license to give and to get...

"After giving talks about open science I've sometimes been approached by skeptics who say, 'Why would I help out my competitors by sharing ideas and data on these new websites? Isn't that just inviting other people to steal my data, or to scoop me? Only someone naive could think this will ever be widespread.' As things currently stand, there's a lot of truth to this point of view. But it's also important to understand its limits. What these skeptics forget is that they already freely share their ideas and discoveries, whenever they publish papers describing their own scientific work. They're so stuck inside the citation-measurement-reward system for papers that they view it as a natural law, and forget that it's socially constructed. It's an agreement. And because it's a social agreement, that agreement can be changed. All that's needed for open science to succeed is for the sharing of scientific knowledge in new media to carry the same kind of cachet that papers do today" (Nielson 2011). 
[T] he work of culture more generally may be seen as training towards letting (things) go. If culture is all about conveying things and skills to others...then learning to let go of things that others appropriately demand is a permanent process. Just as those from whom I have received skills and knowledge (and positions and objects and my life) had to let go of their possessions in the course of their lives, so will I have to learn to let go..." (Widlok 2016).

In this Handbook, there is a lot of description of the future, open academy within a gift economy. If this sounds like an open scientist needs to spend their career giving away their knowledge, that's actually pretty much spot on. But the other, equal, side to this is that an open scientist also gets to get what they need to do their research and build their life. The practice of getting-to-get as a feature of open science culture needs to be explored. What is clear is that this "half" of the gift economy in an abundant open academy is by far the bigger half. It's like putting your potato in the common pot and getting a feast in return. Think of this gift economy as a loan-and-borrow economy, instead of a give-and-take economy. When you share, you also get to keep what you share, and when you borrow, you have no exclusive claim on what you've get: just a promise that there is more out there to use. Below, we will discover how this works.

"Saul Bellow, writing to a friend ... said: 'The name of the game is Give All. You are welcome to all my facts. You know them, I give them to you. If you have the strength to pick them up, take them with my blessing'" (Lethem 2007; Accessed July 20, 2020).

\section{Artists steal, scientists give and get}

In his book Steal like an Artist, Auston Kleon (2012) reminds artists that their lives have been surrounded by art, and that their "original" ideas have been informed in myriad ways by their exposure to this. Stealing is unavoidable, so do it right. Additionally, new art (including music) is always positioned inside of and/or away from the art preceding this. There is an abundance of influences to use, and a debt to all of them. Artists need to be bold and remix what they find, to celebrate the old ideas in their new work. And like science, an artist borrows from the best in order to improve on this using their creative imaginations.

Today, within the academy, stealing practices-the hoarding, scooping, credit-grabbing kinds that are supported by the invented scarcity of ideas and the diminished value for generosity in science-flourish in the absence of social attention and alternative cultural norms. Tomorrow, when open science defines the norms for borrowing, sharing, and celebrated reuse, stealing can be banished to the social margins, and ridiculed as needed.

Borrowing is the main form of sharing in the academy's gift economy. Investigate the meanings and potentials of what you find, and then transform these from those insights 
born of your personal onlyness (See: The Onlyness of the Career Open Scientist). Science is very much like an art here, with a similar relationship to its ideas, but a greater need to maintain the provenance of its knowledge goods. Everything in your science life is borrowed: the knowledge, the methods, every insight up to the point when you add your own, the data you collect. So your job is to learn how to borrow as a scientist.

You are a scientist. You're not agent 007. You are really more like agent $\mathrm{C}_{20} \mathrm{H}_{25} \mathrm{~N}_{3} \mathrm{O}$. But you do have a license. A license to borrow. Come closer. Be honest. You are always on the lookout for ideas worth borrowing. If the journal article you're reading is not worth borrowing, toss it away and keep looking. You are always looking. It's called "research." You hope your own team's ideas are worth borrowing. You make borrowing these easy. It's called "publication." You are a professional thief. You keep careful track of the ideas you borrow. You know where they are from. You've read the articles, you've read the articles they cited. You've read the articles from the citations in those articles. Life would be a lot easier if you could just spin up new ideas on your own. It doesn't work that way.

Borrowing as a scientist in the open-science economy means you get to ask for and take what you need, in culturally specific ways. You take resources to use and reuse, to mine and remix. You pull knowledge from these, and add insights to them in the process. In huntergatherer societies (and sometimes in college dormitories), this is called "tolerated scrounging." In the academy it's more like "celebrated reuse." Open-science research repositories make reuse quick and easy, and they are filled with ideas worth borrowing. And, since these are "non-rivalrous" (See: Neylon 2016), an unlimited number of scientists can borrow them. Better still, the more these ideas are borrowed, the greater their value.

The culturally specific rules for borrowing are being fashioned through the governance processes of scholarly commons (scholarly commons), as these are created to steward common pooled resources toward optimal use. The removal of patents for basic research (See: Hyde 2009; Barnett 2020), is one starting point. Fully public open-access publishing is another. Start somewhere and grow a culture of tolerated scrounging for the resources in your scholarly commons.

"We may consider sharing to be tolerated scrounging but for the scrounging to be tolerated it has to build on a number of recognized modes of action and interaction" (Widlok 2013).

\section{The responsibility is yours, the credit belongs to the whole scholarly club}

$[\mathrm{R}]$ esearchers saw maintaining responsible conduct as the mandatory responsibility of every individual scientist. By choosing this card, the discussants assumed that science's most important responsibility to society was to produce 
reliable knowledge. Research misconduct is then seen as the main threat to this practice..." (Sigl et al 2020).

When you gift (publish) a new scholarly work, you shoulder every responsibility for the rigor in your methods and any issues with your data. This is the first of several social responsibilities (such as mentoring others) you always carry, and one of the keystone virtues the academy has demanded for hundreds of years. Still, you are not the first nor the final author of your own findings. That authority is attached to a thousand places in the prior ideas of others, and in the work of more scientists yet to happen. You merely added one piece to an ongoing solution to the puzzle of nature (or society, etc.): to the "one long experiment" (Martin 1998) that is science. Time to get humble; but if intellectual humility doesn't sound like you, you can claim "hypo-egoic nonentitlement" (Banker and Leary 2019) instead.

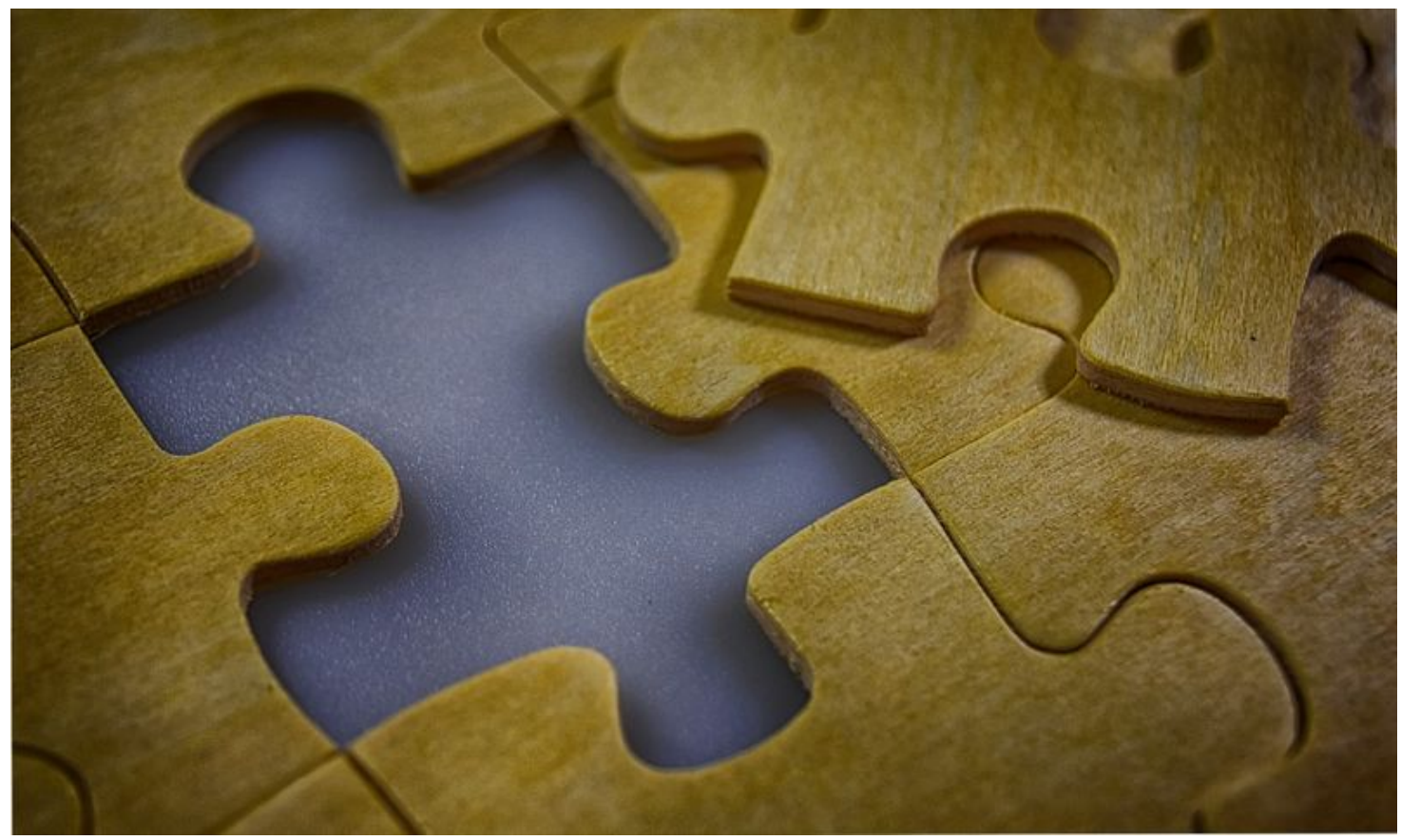

You (and your team) own the event of discovery, but not the piece of knowledge that was produced

Borrowing like a scientist in an open gift economy also means everyone else gets to borrow from you. When they borrow like scientists, this makes you happy. It means your works are borrow-worthy. You celebrate their reuse. In fact you need others to reuse your work to show its reproducibility. Your claim is that anyone would necessarily arrive at the very 
same insight you had, proving that this insight has a durable purchase on its object. If nobody can or does reuse your work, its value is unknown and even suspect.

What is harder to admit is the amount of luck, the confluence of good fortune that brought you to the event where you and your team acquired some new insight (Pluchino A. et al. 2018). Nobody gets to own serendipity. "Serendipity is a category used to describe discoveries that occur at the intersection of chance and wisdom" (Copeland 2019). Riding on the back of the serendipity of reading what you did, talking with whom you have, and trying something new, you've exercised rigor and wonder and perseverance enough through your research to find that one distinct piece of the puzzle to apply it exactly where it fits. Now, you are expected to honor and celebrate the many contemporary and prior ideas that helped you and your team arrive at the singular event within which this new insight was born (See: Shaming the giant). By this, you also show that you belong to the elite club of science. And those who borrow your ideas will honor and celebrate them in theirs.

\section{Commoning needs to get and give to work}

As an open scientist, you have five jobs:

1. produce ideas worth borrowing, and;

2. make these ideas as easy to borrow as possible;

3. borrow as much from other scientists as you need, but borrow like a scientist;

4. become an active maintainer in your commons, to keep the borrowing opportunities rich and rewarding for everyone.

5. as a member of a scholarly commons, you also have the duty to create normative cultural practices to optimize borrowing going forward.

Your list of "good getting" practices will be fashioned to meet the needs of all the commoners in your community. Here's a sample list: 


\section{GETTING}

nead all the work you can in youn field cite as much as possible everything you use

credit goes to all members of youn team emphasize that youn findings are based on the work of others

neuse and credit the methods you've borrowed from a colleague

find someone's data and reuse/rems this with attribution

build on existing open software. enhancing this with new code

work toward badges, not prizes

when offered a prize. insist that the whole team be recognized

\section{STEALING}

grab and publish as original the findings from someone else's work that you've neviewed

take content from a funding neview panel and submit youn own proposal elsewhere

copy ideas from conference posters without attribution

credit youn grad student's work as youn own

demand to be ferst author on a team paper

hoard your own data

insist youn lab-built nesearch software is proprietary

patent youn basic research

Getting practices involve care and attention to the provenance of what's being borrowed, and active credit for those who have made borrowing possible. Stealing practices all point to a game of personal gain based on hiding the sources of your own learning. Getting as a scientist aims to steward the abundance of open resources is a long-term-longer than any lifetime-practice. 


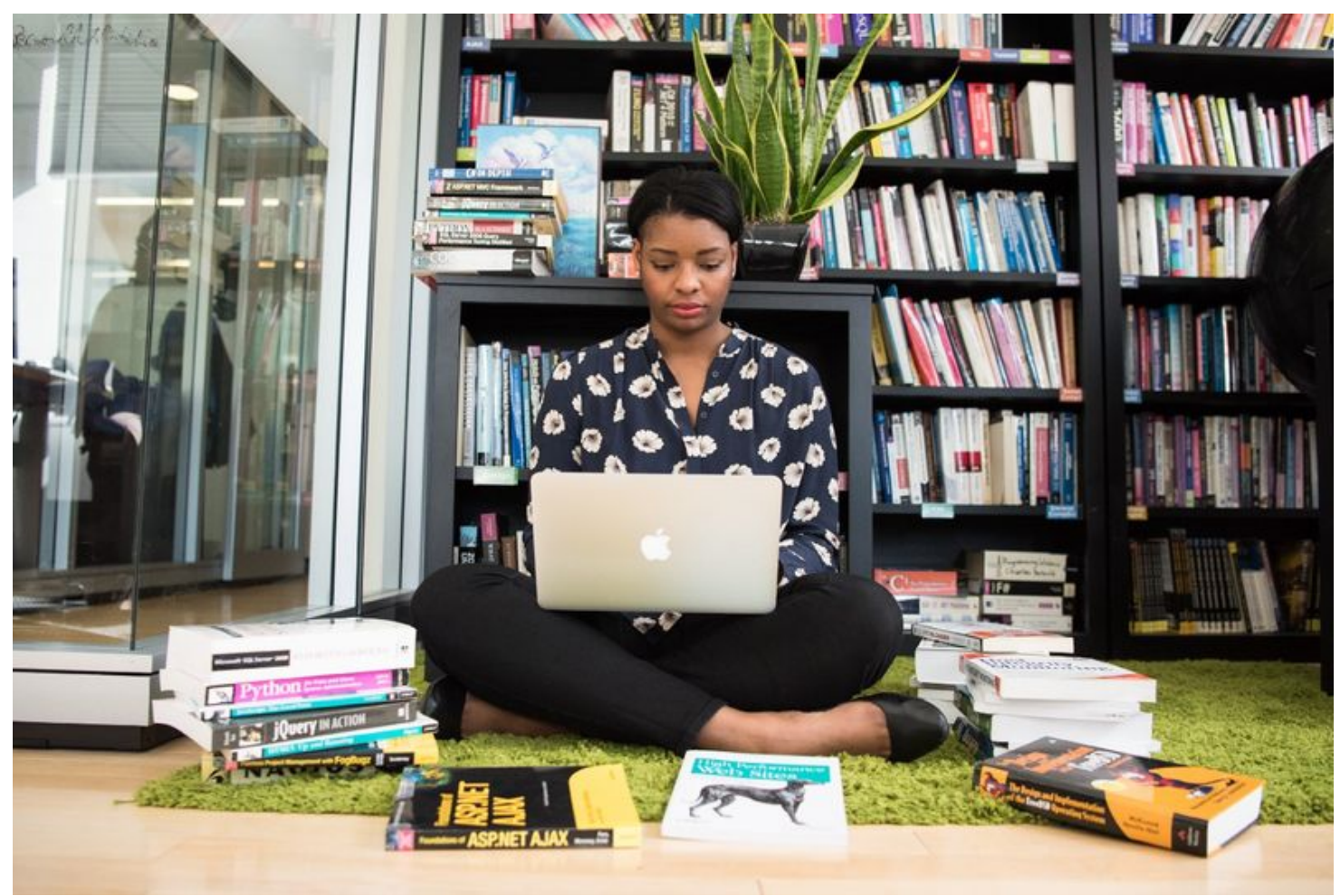

Tolerated scrounging (celebrated reuse) takes time and effort

\section{On disinterestedness: the freedom to discover and share in (open) science}

"Disinterestedness: Scientists are motivated by the desire for knowledge and discovery, and not by the possibility of personal gain.

Self-Interestedness: Scientists compete with others in the same field for funding and recognition of their achievements" (Anderson, et al. 2007).

Let's dig a little deeper into "celebrated reuse" and the history of science. The Mertonian norms of science include a notion of "disinterestedness." At the time Merton was writing, this norm announced a basic freedom to pursue science without conflicts of interests, to shield basic science research from the motivations and (perverse) incentives that come with the marketplace, say, or with other external social/political/military desires. As Vannevar Bush (Accessed August 1, 2020) noted: "Scientific progress on a broad front results from the free play of free intellects, working on subjects of their own choice, in the manner dictated by their curiosity for exploration of the unknown." Disinterestedness is also the culturally valued attitude of "letting go" when others build on your findings.

Disinterestedness was, and still is, the classic norm that frees you to let others in the academy club borrow your work. The same lack of self interest that validates your 
independent research choice also validates you being able to let other people freely use your work. Since you chose to not let external interests determine your research path, you have no reason to hoard the results.

Disinterestedness is one of the social costs of academic freedom (you probably can't have one without the other). It is the reason why the imbalance between responsibility (you have $100 \%$ of this) and authority (you have very little of this) makes perfect sense. You take the freedom to choose your research path in exchange for gifting the results back to the community; you release your personal interest in these results to benefit the whole scientific club.

If self-interest is your main incentive to do science, you are not doing open-science. Worse than that, you are doing science wrong. If you decide to wait until you have tenure to throw off self-interest (Anderson et al 2010), you are also doing it wrong. Certainly, we all have a stake in our own interests. Science expects us to care for these interests outside of our scientific explorations, and we need institutional reform and support to get there. But mainly, disinterestedness tells us to avoid conflicts with interests from outside the "republic of science" (Polanyi 1962).

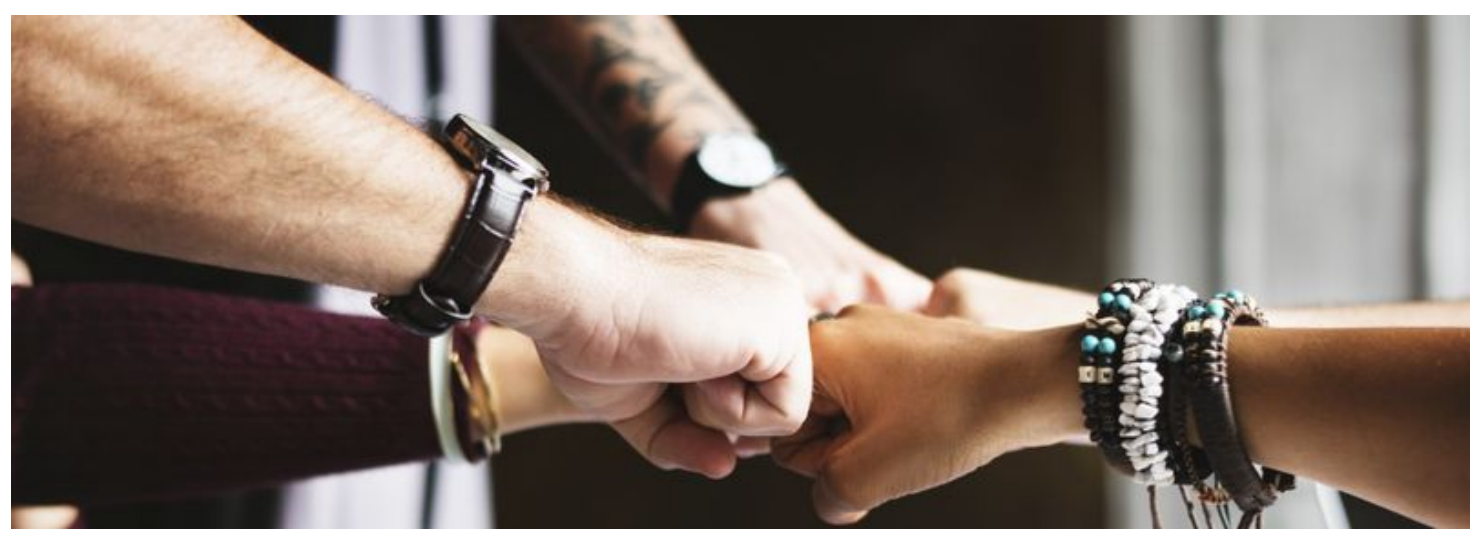

\section{Kindness and care are not optional}

"[M] uch of academic thinking brackets issues of emotions and values outside of academic understanding, even though emotions and values inhabit research and teaching by virtue of what we know, what we choose not to know, what we prioritise and what we trivialise" (Lynch and Ivancheva 2016).

Research needs to be free to follow its own objects, but being passionate about your research is no free ticket to act poorly with others (See: Six rules about passion in the workplace). Not caring about the marketplace and not caring for your colleagues are two different practices. 
Disinterestedness is not an alibi to ignore/resist other organizational cultural values for the academy, values that include kindness and care in academy workplaces and in relations with colleagues. You can start by bracketing out the perverse marketplace incentives that might warp your research path and diminish your own passion for the pursuit of science. Passion is another part of science that is not peculiar to you. You are not the only person in the room or on your team that has been infected with the intellectual disease of science. This is a long-term global knowledge pandemic. Everyone gets to be infected-to be passionate-in their own way. Exploring these passions through years of rigorous research effort builds a kind of shared practical wisdom inside the profession.

\section{Applied practical wisdom: the practice of open science}

The practical wisdom that underpins actually doing science removes the need for other incentives. The answer to the question: "How do you incentivize scientists to do research and teaching?" is simply this: "give them more opportunities to learn the practical wisdom required to do science" (See: The practical wisdom of science praxis). Science requires/ rewards its own unique practical wisdom. In addition to the practical wisdom one might (and perhaps should) acquire through social experiences with others (colleagues, family, strangers), doing science offers opportunities to acquire practical wisdom through a career experiencing nature as a complex emergent system.

For many years, you borrowed learning from your teachers. Now, you encourage your students to scrounge new knowledge. Today, you borrow like a scientist: information from your objects of study and insights from conversations with your colleagues. In tomorrow's open-science culture, culturally-informed practices for getting-to-get will help you and your team and your organization optimize the use of the emergent scholarly commons infrastructure and content. The work needed to articulate and support these practices will be significant. But know that the work needed to support stealing in the academy today is just as arduous, except that so many academics have already learned how. Unlearning these toxic cultural practices will take time and reflection.

Today, dozens of open-science platforms and communities are encouraging effective reuse. Reuse is one metric that deserves to become a goal (and one goal that makes a handy metric). How does your organization, your discipline, or your team celebrate active reuse? Where can it improve? 


\section{Demand sharing and the power of pull}

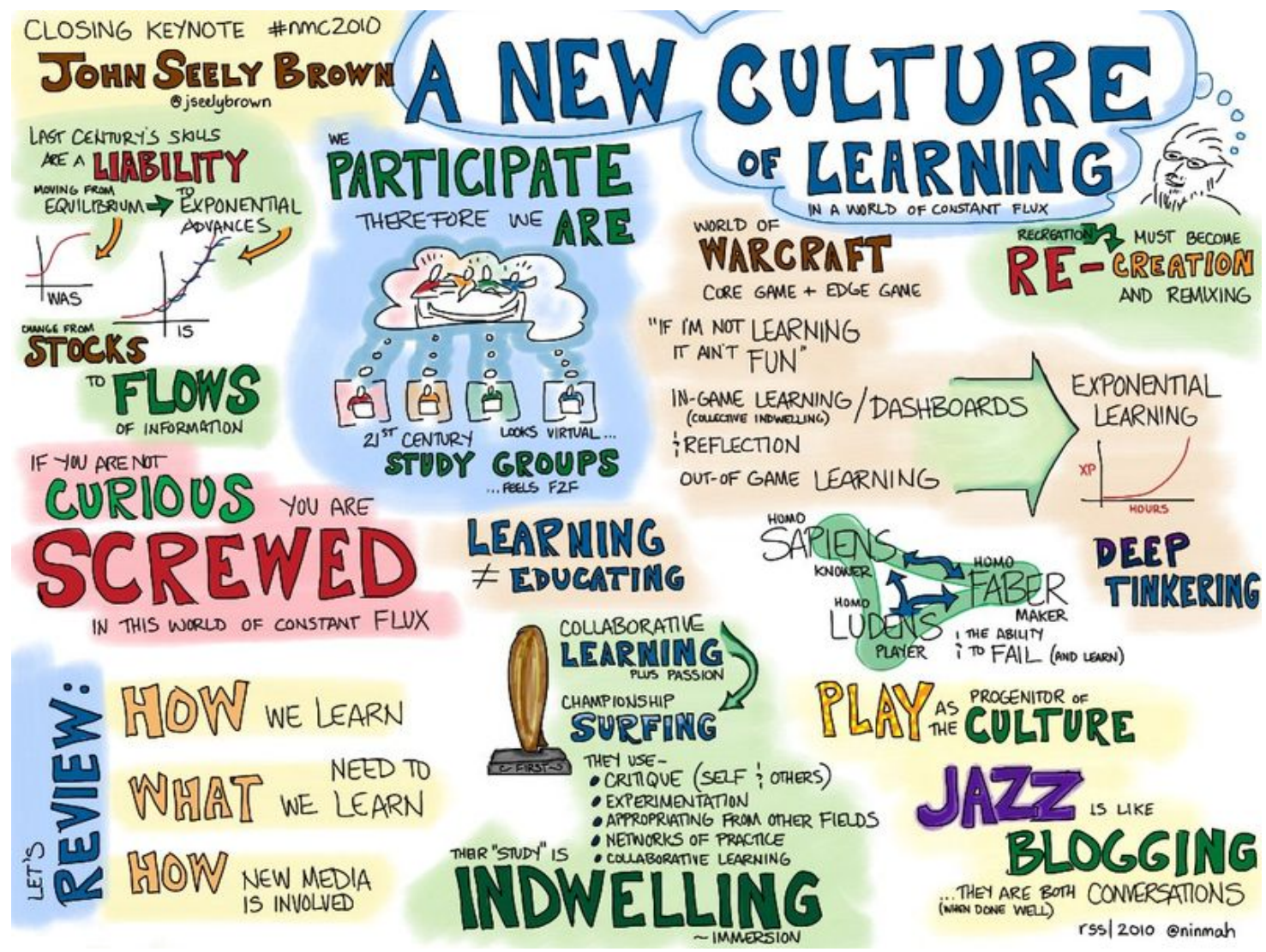

Credit: Rachel Smith on Flickr CC. Notes from a John Seely Brown talk

\section{Unleash the "power of pull" for your science research}

"In a closed society where everybody's guilty, the only crime is getting caught. In a world of thieves, the only final sin is stupidity" (Thompson 1971).

"Pull allows each of us to find and access people and resources when we need them, while attracting to us the people and resources that are relevant and valuable, even if we were not even aware before that they existed" (Hagel 2010; Accessed February 26, 2020).

\section{"Sharing" gone massively wrong: academic publishing}

Why is Demand Sharing so important to open scientists like you? We are going to explore this question here. Let's begin with the poster-child for research-sharing-gone-wrong: "for- 
profit science publishing." At the same time you've been perfecting your demand-sharing techniques in the classroom, you've surrendered your research to one of the strangest marketplace transactions in modern times.

Academics give their research to publishers; give the publishers their copyrights; and also donate an additional sixty-eight million hours a year (Nature News Sept 7, 2018: $<$ https: // www.nature.com/articles/d41586-018-06602-y>) reviewing the work of others for free. Academic libraries must each pony up millions of dollars a year to keep their subscriptions current. The public gets dinged thirty to forty dollars (US) a pop just to read a single article. The process of selecting articles often leads to months or years between discovery and publication, and warps the output toward positive (and false-positive) "sexy science" results. The remainder of research results go unpublished. "Economists may not have terms adequate to describe a market as dysfunctional as the one operating for academic publishing" Neff (2020; Accessed February 26, 2020) notes.

\section{How did this happen?}

Potts (et al. 2017) points to a failure of the publishing capacity of academic societies to scale with the blossoming of science output after World War II:

"The wartime and post-war expansion of public research funding and consequent expansion and globalisation of research communities were soon exploited by an entrepreneur-led proliferation of increasingly specialised journals, following the lead of Robert Maxwell's Pergamon Press (Buranyi 2017). The small society presses, struggling to cope with growing scale, were supported and then largely supplanted by the 'Big 5' commercial presses: Elsevier (which acquired Pergamon in 1991), Wiley, Springer, Taylor \& Francis and Sage. These newly-empowered players brought an industrial approach to the publication and dissemination process, for the first time realising the benefits that these specialised capital and skills could provide by operating at a scale that was unprecedented to that date. The successful publishers grew (and consolidated to grow further) alongside a pre-Cambrian explosion and specialisation of journals to create the modern landscape in which the majority of journals is owned, controlled or at least produced by a handful of globalised companies."

A committee at the National Academies of Sciences (2018) offers additional historical information:

"The 1990s brought a wave of consolidation among scientific publishers, as Netherlands-based Elsevier acquired Pergamon, leaving it in control of over 1,000 journals (Buranyi 2017 [ibid]). Further increases in subscription prices and the advent of "big deal" agreements between publishers and libraries followed in the 
late 1990s. Under these agreements, publishers agree to provide online access to a bundle of their journals, including all back issues, priced at a discount to the sum of the individual journal subscriptions (Bergstrom et al. 2014). Despite paying lower per journal prices, total outlays by libraries increased to the point where this has been called the "serials crisis" (Panitch and Michalak 2005[white paper is no longer online]). In 2015, Larivière et al. found that the five most prolific publishers, including Reed-Elsevier, Taylor \& Francis, Wiley-Blackwell, Springer, and Sage, control over one-half of all the scientific journal market, and that the profit margins of these companies have been in the range of 25 to 40 percent in recent years (Larivière et al. 2015). According to one economist who studies the industry, this situation 'demonstrates a lack of competitive pressure in this industry, leading to so high profit levels of the leading publishers that they have not yet felt a strong need to change the way they operate' (Björk 2017a)".

Both of these accounts point to older, established cultural practices based on demand sharing within the academy being disrupted and displaced by marketplace profit seeking. By the end of the Nineteenth Century, the academy had taken control of its own research sharing practice through the advent of hundreds of member-run professional societieseach with their own publishing effort. Within these societies, members freely gave up their research for review and publication. University presses added their capacity as well.

\section{In demand sharing, a "demand" is a culturally-grounded request}

As academic institutions, the societies and universities demanded research finding from their members, in much the same fashion that students can demand knowledge from their professor in the lecture hall. Sharing is both expected and normative. Merton (1973) noted that:

"The institutional conception of science as part of the public domain is linked with the imperative for communication of findings. Secrecy is the antithesis of this norm; full and open communication its enactment. The pressure for diffusion of results is reenforced by the institutional goal of advancing the boundaries of knowledge and by the incentive of recognition which is, of course, contingent upon publication."

Societies provided both the means of building the shared, public, academic corpus, and the platform for recognition. Yet this individual recognition was also tempered with the larger sense that all knowledge is interlinked and historically accumulated. Merton (ibid) writes: "The communal character of science is further reflected in the recognition by scientists of their dependence upon a cultural heritage to which they lay no differential claims."

\section{Demand sharing on the open web}


Open science efforts in the last twenty years have been centrally focused on refactoring the means of academic publication to take advantage of the opportunities provided by the internet, and to remove the foreign, marketplace, logic in order to reassert the "communal character" of science publishing, grounded in the logic of demand sharing (although they haven't called it that). Unlinking the act of giving research results back to the science community - which has long been a community norm - from the more recent practice of giving away research results to the marketplace - to own from there forward-restores these results as internal "gifts" within a community guided by demand sharing. There is more. At the same time that open science releases the academy from its recent marketplace bondage (freeing up financial resources in the process), a new, networked marketplace for "ideas" in and out of the academy is also challenging the notion of organizational knowledge ownership, in favor of what Hagel (et al. 2012) calls the "power of pull."

Large corporations, fledgling start ups, and, yes, even ivory tower universities can access an explosion of shared knowledge and lateral learning when they decide to pull information from global networks. "Institutions can significantly amplify the power of pull, making it far easier to connect with a broader range of people and resources and to learn faster from each other than we ever could in the absence of institutions. We must therefore reclaim our institutions - whether from the inside of existing ones or by creating a new generation of our own." (ibid) Open science works to reclaim the academy as learning hubs that can pull information from academy commons resources across the planet.

Goldman and Gabriel (2005) observed that "innovation happens elsewhere"; that the crowd- and network effects of open communities could assemble more talent, a greater variety of knowledge, and effective collective intelligence(s) well beyond those that any company/university/lab could afford to assemble internally. Their arguments were informed by Bill Joy, a co-founder of Sun Microsystems, who wrote in the 1990s: "no matter who you are, most of the smartest people work for someone else" (Wikipedia). This is known in management theory as Joy's law. And it holds ever more strongly for your university, your agency, or your laboratory.

Many corporate management experts point out that openly sharing ideas across corporations (Golden and Gabriel 2005; Leadbetter $2005<$ https://www.ted.com/talks/ charles leadbeater on innovation?> Accessed April 21, 2019), and gathering ideas from customers and external sources (Bissola et al. 2017), will lead to better, faster corporate innovation. In fact, you can say, with some authority, that the future of innovation in the academy will require the logic of demand sharing.

The marketplace logic of intellectual property ownership and practice of demand sharing for knowledge are antithetical. They do not play well together. For-profit efforts to include "open practices" invariably lead to open-washing, and the final closure of collected resources as intellectual property (Neylon 2017). Open for them means "free to acquire" 
and mainly serves to lower their resource costs. Demand sharing is an older practice, older in the academy, and still older in the species, being a primary form of cultural practice over thousands of years. It privileges internal goods over the external goods and incentives of the market.

To understand this further, jump to (See: Gifting and Reciprocity), where you can explore how each act of demand sharing builds a social bond that can be used to stimulate other occasions of sharing. The academy also needs to break completely from seeking to own intellectual property inside individual organizations (Against Patents in the Academy) in favor of academy-wide ownership supporting a shared resource commons for intellectual property. Current work on the creation of "Civic Trusts" offers a productive way forward for the academy (See: The Civic Trust; Accessed February 26, 2020). 


\section{A logic of abundance}

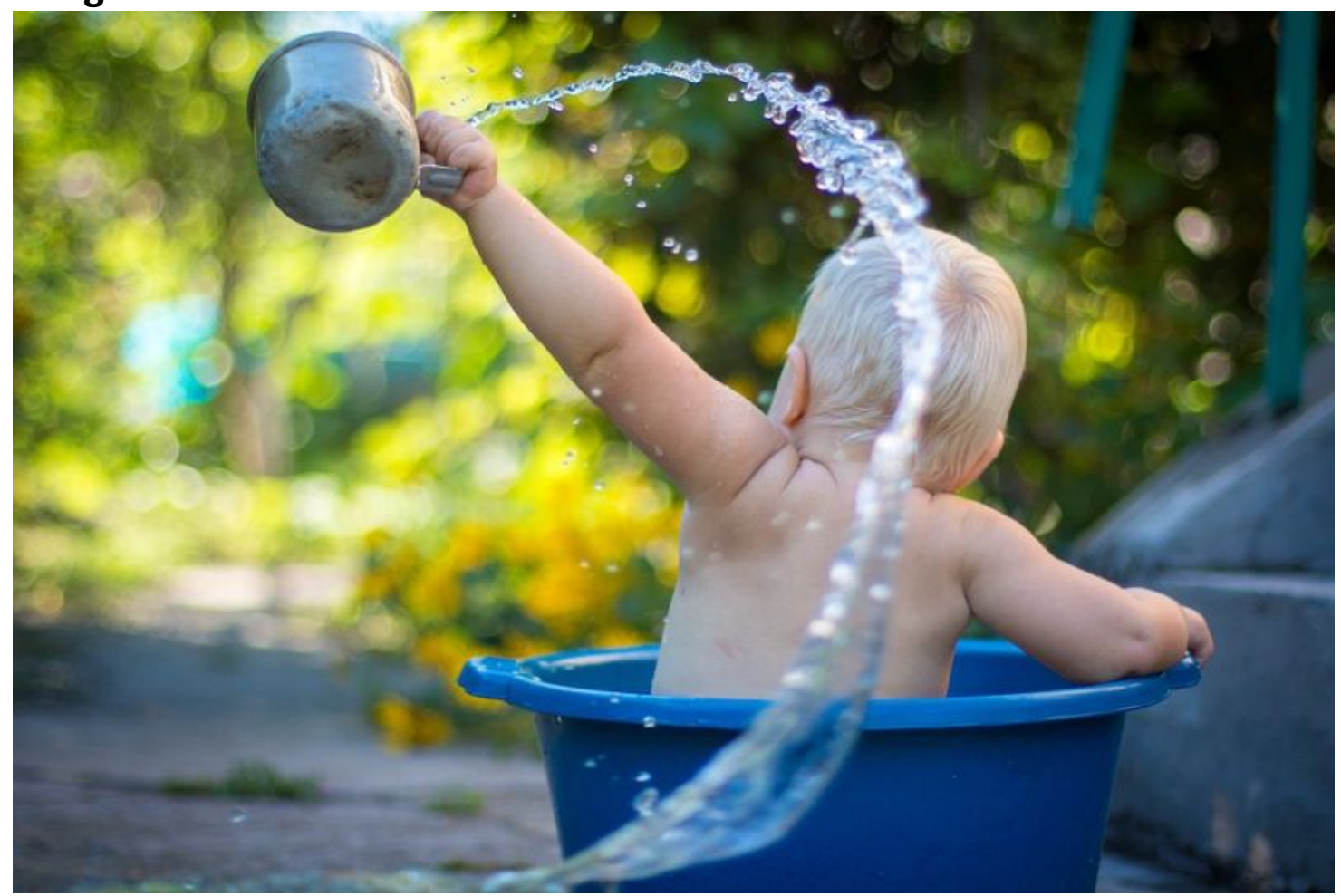

How big is your bucket? Make it smaller to find "enough".

\section{Get Ready for Abundance}

"The opposite of scarcity is not abundance. The opposite of scarcity is... enough" Brené Brown Podcast <https://youtu.be/YxAcUOelnAY>.

"Scarcity is easier to deal with than abundance, because when something becomes rare, we simply think it more valuable than it was before, a conceptually easy change. Abundance is different: its advent means we can start treating previously valuable things as if they were cheap enough to waste, which is to say cheap enough to experiment with. Because abundance can remove the trade-offs we're used to, it can be disorienting to the people who've grown up with scarcity. When a resource is scarce, the people who manage it often regard it as valuable in itself, without stopping to consider how much of the value is tied to its scarcity" (Shirky 2010).

"For the Ju/'hoansi, that fundamental axiom of modern economics, "the problem of scarcity", simply did not apply. Where this holds that it is human nature to have 
infinite wants and limited means, the Ju/'hoansi had few wants that were simply met" (Suzman 2017 [The Guardian]).

\section{Abundances}

We have not really begun to explore the many varieties of abundance that can emerge once we abandon arbitrary scarcity. It is important to remember, as Brené Brown (2012) reminds us, that abundance is also simply enough: enough access and discovery tools to know your research is new and where it sockets into existing knowledge; enough communication with colleagues working in your specific arena to collaborate your way through pain points; enough research funding to move ahead; enough time to do your work, and; enough credit to know your work is out there and appreciated. Enough is never too much. It is all the abundance an open scientist needs.

Primary abundance is built into digital science objects which, like Jefferson's thoughts, can be copied infinitely without diminishing the original. Quite the opposite, the more copies that circulate, the more valuable the original object becomes, only not as the private property of an individual, but rather as a common pool resource for the commons.

Combinatory abundance is what happens when science objects (and scientists) enter into collaborative matrix to mix, meld, and produce new objects. This is also where the network effect applies to objects, not just people.

"The difference between humans and animals lies in the ability to collaborate, engage in business, let ideas, pardon the expression, copulate. Collaboration has explosive upside, what is mathematically called a superadditive function, i.e., one plus one equals more than two, and one plus one plus one equals much, much more than three. That is pure nonlinearity with explosive benefits-we will get into details on how it benefits from the philosopher's stone." (Taleb 2012) paraphrasing Ridley (2010).

Language is a good example of the kind of combinatory abundance that open science hopes to achieve through mineable/mixable repositories of a wide variety of knowledge objects. The English alphabet has twenty-six letters and the English language about forty phonemes. From these all words, sentences, paragraphs, texts, and conversations are spun by combining and assembling them using rules and shared semantics.

You're an academic, you know that academics might run out of patience, or time, or even wine, but rarely do we run out of words (or ideas for that matter). In fact this is one 
abundance that we have always enjoyed, perhaps a bit too much. To achieve the "explosive upside" of collaboration, scientists need to build open cultures of collaboration.

Emergent abundance describes the complex objects of study, the unknowns that feed science and also science's willingness to not seek "truth". Whether you are tracking the micro-second changes of a single cell or the collision courses of galaxies, you begin with a never-decreasing abundance of questions. Science also has an abundance of doubts, as well as discoveries. Science swims in an ocean (an abundance also) of doubt, as Richard Feynman reminds us: "A scientist is never certain. We all know that. We know that all our statements are approximate statements with different degrees of certainty; that when a statement is made, the question is not whether it is true or false but rather how likely it is to be true or false" (Feynman 2005).

What emerges from these doubts is a collective form of being only slightly less...wrong. Being less wrong iterates into being somewhat more right, but never to the point of actual truth. Everything we know today will be different from what we know tomorrow.

"[S]cientists gravitate toward falsification; as a community if not as individuals, they seek to disprove their beliefs. Thus, the defining feature of a hypothesis is that it has the potential to be proven wrong (which is why it must be both testable and tested), and the defining feature of a theory is that it hasn't been proven wrong yet. But the important part is that it can be - no matter how much evidence appears to confirm it, no matter how many experts endorse it, no matter how much popular support it enjoys. In fact, not only can any given theory be proven wrong; ... sooner or later, it probably will be. And when it is, the occasion will mark the success of science, not its failure" (Schultz 2011).

Infinite abundance marks the recognition that science is not a finite game. There is no way to "win" science; no ending of science; and no possibility for its rules to be fully known; these are continually subject to change. The great mistake of bringing the logic of the marketplace (a finite, zero-sum game) into the academy is that it promotes behaviors that treat science like a finite game, and it makes competitors out of colleagues.

As a form of infinite play, science finds itself in a never-ending tussle with its objects of study; "Our freedom in relation to nature is not the freedom to change nature; it is not the possession of power over natural phenomena. It is the freedom to change ourselves. We are perfectly free to design a culture that will turn on the awareness that vitality cannot be given but only found, that the given patterns of spontaneity in nature are not only to be respected, but to be celebrated" (Carse 1987). James Carse's book, Finite and Infinite Games, offers a great heuristic for the type of culture change needed for science to become "open science" 
"THERE ARE at least two kinds of games. One could be called finite, the other infinite. A finite game is played for the purpose of winning, an infinite game for the purpose of continuing the play."

...

..."It is on this point that we find the most critical distinction between finite and infinite play: The rules of an infinite game must change in the course of play. The rules are changed when the players of an infinite game agree that the play is imperiled by a finite outcome-that is, by the victory of some players and the defeat of others. The rules of an infinite game are changed to prevent anyone from winning the game and to bring as many persons as possible into the play." (Carse 2011)

Sufficient abundance reminds us that abundance does not need to be a waterfall into an overflowing bucket. As long as the bucket is full, there is abundance. A single extra drop makes it overflow. Abundance is relative to needs, and needs can be managed to the level of sufficiency, rather than expanded by market-fueled desires. In reality, abundance just means this: enough.

Open science advocates are often asked about how they will replace (perverse) market incentives; as if these are the only incentives out there. Scientists have their own incentives, the reasons they are scientists and not, say, hedge fund managers. And scientists were fully incentivized in the decades before the marketplace intruded on the academy. There are many articles about the mismatch between science and market incentives. A good place to start is Edwards and Roy (2016):

"In this article, we will (1) describe how perverse incentives and hypercompetition are altering academic behavior of researchers and universities, reducing scientific progress and increasing unethical actions, (2) propose a conceptual model that describes how emphasis on quantity versus quality can adversely affect true scientific progress, (3) consider ramifications of this environment on the next generation of Science, Technology, Engineering and Mathematics (STEM) researchers, public perception, and the future of science itself, and finally, (4) offer recommendations that could help our scientific institutions increase productivity and maintain public trust. We hope to begin a conversation among all stakeholders who acknowledge perverse incentives throughout academia, consider changes to increase scientific progress, and uphold "high ethical standards" in the profession..."

Offer a scientist more time, cheaper tools, and some security to finish their research, and you will have a happy scientist. Chasing reputation points and writing endless proposals for funding would not compete with simply clearing the decks and letting research come to the fore. Managing needs can be a productive alternative to bulking up the CV with marginal 
publications. Open science can wean the scientist from perverse incentives by offering more with less.

Are you tired of working so hard to get just a bit more? One of the tasks of open science is to innovate to lower the costs of doing science. The most "successful" societies in the history of humanity became affluent by managing their needs:

"[Marshall] Sahlins characterized hunter-gatherers as the gurus of a "Zen road to affluence" through which they were able to enjoy "unparalleled material plentywith a low standard of living." Here, it seemed, was a people unconcerned with material wealth, living in harmony with their natural environments, who were also egalitarian, uncomplicated, and fundamentally free" (Suzman 2017).

Sometimes one can achieve abundance by simply finding a smaller bucket. Matching needs to resources (instead of the other way) can tip your situation from scarcity to "just enough".

\section{The next steady-state of science is the provident abundance of resources}

After decades of scarcity, real and imaginary, and of regimes of funding that encouraged hyper-competition, the move to a research eco-system of abundance will necessitate new practical knowledge to navigate and optimize this. At some point, scientists will become comfortable knowing that they can find and demand what they need to do their work. They will become affluent academics: rich in the resources they require, even as they are detached from marketplace incentives. Like hunter-gatherers, their ability to glean what they need means they no long have any reason to hoard what they have.

"In part, the Ju/'hoansi's affluence was based on their unyielding confidence in the providence of their environments and their skills at exploiting this. Ju/'hoansi still make use of well over 150 different plant species, and have the knowledge to hunt and trap pretty much any animal they choose to. As a result, they only ever worked to meet their immediate needs, did not store surpluses, and never harvested more than they could eat in the short term" (Suzman 2017 [The Guardian]).

Building provident academic resources (repositories, metadata, platforms, etc.) for reusable digital objects, global in scale but also localizable for use, is a major task for open science. The enormity of this task has occupied a lot of attention in the past decade. National and international data organizations, and professional associations and universities (with important nudges from funders) are working on parts of this task.

There is an equally important task that open scientists need to tackle: Governing these resources and forging the shared social practices that can do so. This second task represents a major cultural challenge for the open science academy, and for each part of 
this (academic departments, research labs, professional associations, universities, funding agencies); and, finally, for each individual open scientist. Culture is aways shared, and always carried by individuals.

Scarcity, as we will see next, is mainly manufactured to game values in the marketplace. If you make less of something, when the demand is constant, you an increase the price.

\section{Final thought: Abundance is how you perceive your work.}

"If you perceive the universe as being a universe of abundance then it will be. If you think of the universe as one of scarcity, then it will be. ... I always thought that there was enough of everything to go around-that there are enough ideas in the universe and enough nourishment." Milton Glaser in (Millman 2007).

\section{Scarcity is mostly manufactured and arbitrary}

"I am often told that I should be grateful for the progress that Western civilization has brought to these shores. I am not. This life of work-or-die is not an improvement on preinvasion living, which involved only a few hours of work a day for shelter and sustenance, performing tasks that people do now for leisure activities on their yearly vacations: fishing, collecting plants, hunting, camping, and so forth. The rest of the day was for fun, strengthening relationships, ritual and ceremony, cultural expression, intellectual pursuits, and the expert crafting of exceptional objects. I know this is true because I have lived like this, even in this era when the land is only a pale shadow of the abundance that once was. We have been lied to about the 'harsh survival' lifestyles of the past. There was nothing harsh about it. If it was so harsh — such a brutish, menial struggle for existencethen we would not have evolved to become the delicate, intelligent creatures that we are" (Yunkaporta 2020).

"As Lawrence Lessig has so persuasively argued over the years, there is nothing 'natural' about the artificial scarcity of intellectual property law. Those laws are deliberate interventions crafted by human intelligence and are enforced almost entirely by non-market powers. Jefferson's point, in his letter to McPherson, is that if you really want to get into a debate about which system is more 'natural,' then the free flow of ideas is always going to trump the artificial scarcity of patents. Ideas are intrinsically copyable in the way that food and fuel are not. You have to build dams to keep ideas from flowing" (Johnson 2011). 
Ideas are not like food, nor fuel. But then the scarcity of jobs, careers, and funding is not simply artificial either. Scarcity is real, you might say. However real this scarcity is today, it is not necessarily as durable as you think. So, how does open science fuel a new abundance for these various resources?

Let's start with the problem. Scarcity is created to build markets where arbitrary values can be maintained to guarantee a profit. Widlock describes it this way: "Modernity defines itself as a 'culture of scarcity'..., of there never being enough goods and information. The assumed 'eternal shortage' paradoxically seems to grow, rather than to diminish, as modernity and capitalism unfold and ever more consumer needs are created. The underlying orthodox assumption is that economy is above all economizing (making ends meet) and directed toward utility as being conditioned by limited means employed to satisfy unlimited wants." (2016) Of course, the "wants" are also manipulated by the marketplace: they are attached to the outcomes of the scarcity machine.

Bauwens (2012) adds that markets cannot survive without scarcity: "Markets are defined as ways to allocate scarce resources, and capitalism is not just a scarcity 'allocation' system, but, in reality, is a scarcity engineering system, which can only accumulate capital by constantly reproducing and expanding conditions of scarcity. When there is no tension between supply and demand, there can be no market, and so no capital accumulation" $<$ http://realitysandwich.com/142773/evolving partner state ethical economy/> Accessed 10/2018). Markets only succeed when they can manufacture enough scarcity to raise the price of what they manufacture high enough to make a profit; they have the means to make enough of the goods to drive the price down, but chose not to do so (Siefkes 2008).

The market logic of scarcity, and the resulting neoliberal push to remake the academy as a business venture has been a uniform disaster for universities, and an existential threat to the reputation of science. Science findings that are infested with conflicts on interest create doubt in the public. When scientists are suspected of not being truthful, of not upholding the primary sincerity required for the scientific method to stand, then their findings, even when these might be true (to the amount this is available), will be met with doubt. This impacts the ability of the public to rely on science for their own sense-making, and undermines science's position in the public information ecology (See: The War on SenseMaking; Accessed 10/31/2020).

Open science alone cannot reverse this trend. Expecting open science to fix the academy without first extracting this from a hyper-rivalrous economy puts too much on the back of this cultural shift. But the practices of open science can offer expanded returns on the investments the state makes into the academy. These practices optimize the value of research objects and reground science by its internal norms. The culture of open science can help return the academy to a value proposition that centers research and teaching as a public good that reaches toward social justice and broadly-felt benefits across society. 
Open science values contributions from a broader cohort or intellectuals. Doctorate holders represent a tiny proportion of the planet's population (less than $2 \%$ ). But the problems the world faces are more complex and numerous than ever before. "There is, however, no limit on society's need to address complex challenges, the number of research questions that can be asked, or the amount of scientific work that can be done. New models are needed to help identify different ways for scientists to continue their work outside of a standard academic or agency job" (Lancaster, et al. 2018).

\section{Right now is the time to euthanize Matthew}

Open science culture prioritizes widespread, global participation, which would necessitate redistributions of academic fortunes away from current elite organizations and funderfavorite research endeavors: the result of decades of cumulative advantages, sometimes called the "Matthew Effect." The process of "un-accumulating" advantages in the academy will be painful, particularly to those who have invested in them.

Today, "[d]ifferences in individual capabilities aside, then, processes of cumulative advantage and disadvantage accentuate inequalities in science and learning: inequalities of peer recognition, inequalities of access to resources, and inequalities of scientific productivity" (Merton 1988). Tomorrow, an open-science academy will need to refactor decision processes to spread around resources that are currently limited, and challenge the current level of available funding by making the case that it can optimize the return on the value-including a wide range of social benefits-for public investment in the public goods of science.

"The ecosystem metaphor is our attempt to reframe and expand the discussion of STEM careers and science beyond what has often become a sterile and arid debate about competition and scarcity within academia by connecting it with the open flows, resilence, diversity and feed-back loops of ecological systems. ...[W]e strongly believe that making science better is not just 'making the incentives better', but a collective cultural shift beyond viewing competition and individualistic success as the sole defining feature of science (i.e., the pipeline model)" (Lancaster et al. 2018).

Instead of an economy that relies on hyper-competition to create an underclass of $\mathrm{PhDs}$ (Chapman, C.A., et al. 2019) for hire at almost any price, an open, sharing academy economy would find guaranteed employment for all PhDs at one of the many available academy institutions, or elsewhere in society (See: Tcherneva (2020) on the benefits of job guarantees). 


\section{Idea Farming}

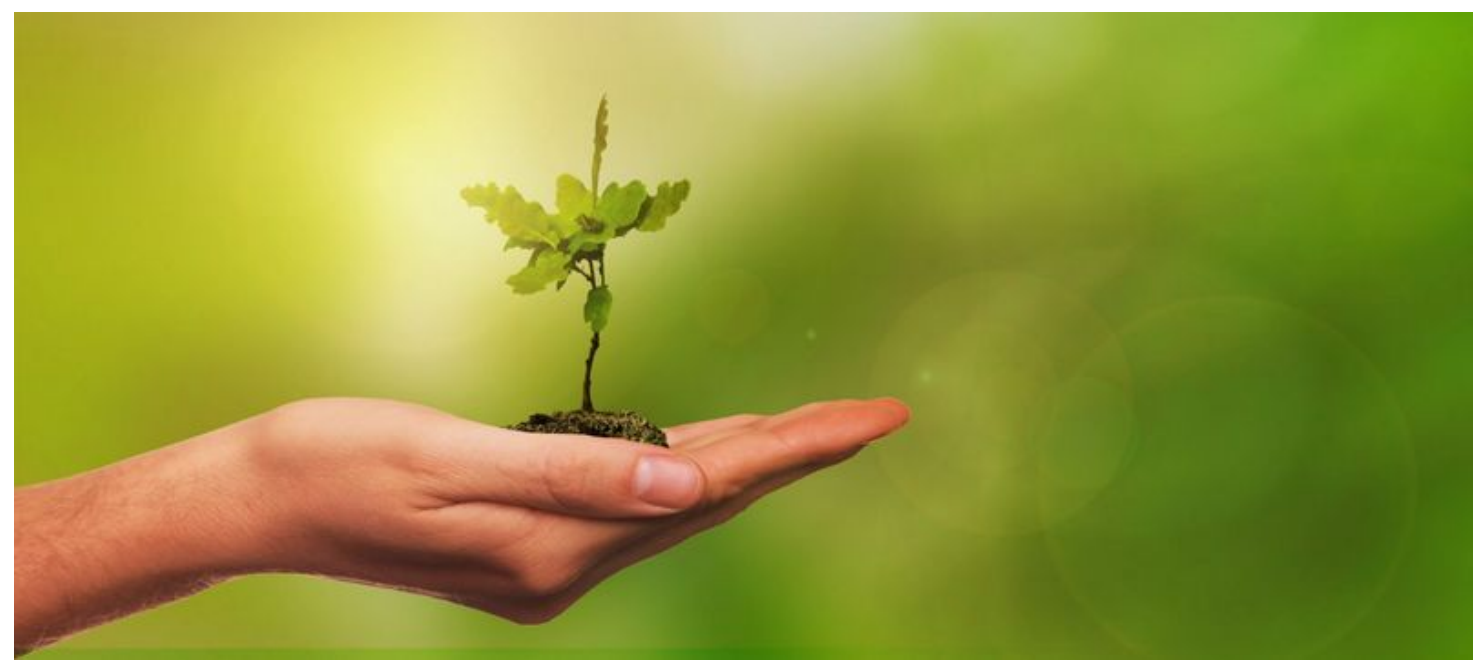

You need to plant it for your idea to grow

\section{Open science means leaving the idea desert for the idea garden}

Most often, new discoveries and new learning come when one is open to serendipity, when one welcomes novelties and anomalies, and then tries to incorporate those outlying results into the broader field of knowledge. As Isaac Asimov said, 'The most exciting phrase to hear in science, the one that heralds new discoveries, is not "Eureka!" but "That's funny"' (Brown 2009).

Ideas in the academy are another victim of the logic of arbitrary scarcity. They are also collateral damage in the proximity to the neoliberal, start-up economy. The academy should be an idea hot-house, instead we have an idea desert. The cultural shift to open science will be final when ideas flow across the globe like pinot at a faculty party.

\section{Common sense on idea sharing}

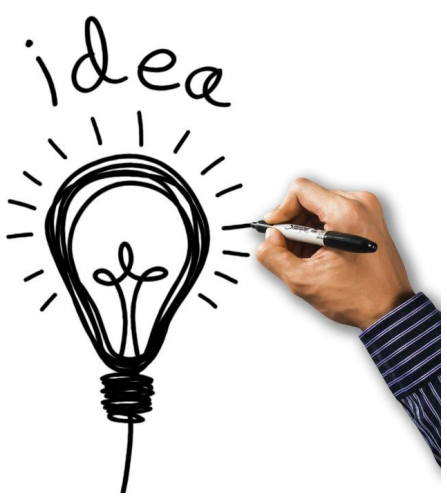

\section{Turn your ideas into citations}

Like talk, ideas are cheap. How many research ideas do you have in an hour? In a day? In a week? Probably enough that you really wouldn't take time to even jot them down. There are the ideas that seek to connect your current work to new hypotheses. Ideas about alternative methods, or new 
data sources. So many ideas crowd into your thoughts:

- Ideas you have while listening to a seminar talk outside your field, where you are curious how something you know might be of use or interest;

- Ideas on where your discipline is headed, and those large-scale issues that might drive agency agendas;

- Ideas that pop up when you read that new journal article (any article that does not give you new ideas is a waste of your time);

- Ideas about what your graduate students might want to pursue to start their own infinite play in science;

- Ideas you put into that NSF proposal you submitted last month;

- Ideas.... Well; you get the idea...

Face it: your professional life is brimming with ideas; that's pretty much the point. And yes, you don't want or need to share them all. Over time, you will get better at triaging the insights that occur to you. Ideas are the starting line for infinite play (See: Learning infinite science play). There are about ten million science researchers on the planet. Each of you wakes up to a new day filled with new ideas. Almost all of you keep most of your ideas in your head until they are forgotten, replaced with other ideas, similarly forgotten, and a couple insights, carefully hidden in a notebook or on a laptop. We live in a world where there is no lack of abundance of good ideas in science. We also live in a world with a global internet. Why not connect these two? That's an idea.

Note: thoughts are not ideas. Thoughts are just thoughts; you have a steady stream of these, most of which you would not share without first being injected with Sodium thiopental. Also, nobody wants to hear your thoughts. 


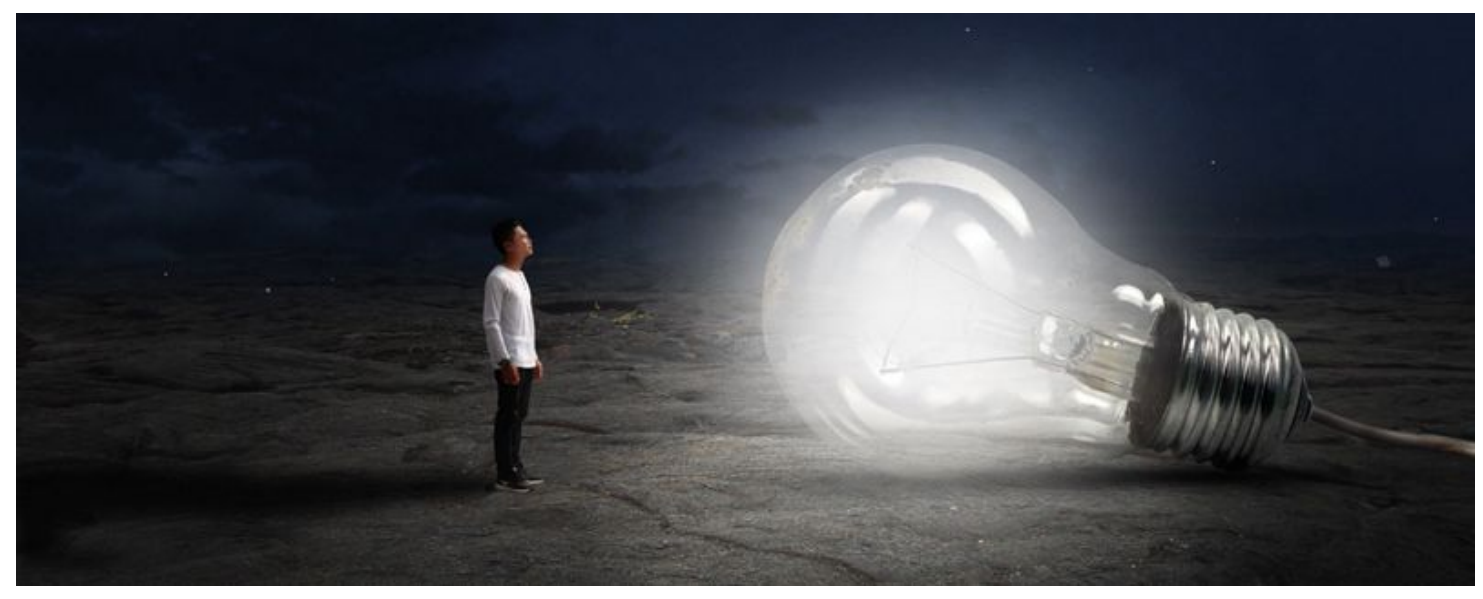

When asking scholars about their ideas, some common notions arise:

1.) Even though that idea you just had while brushing your teeth might seem unremarkable to your own work and aims, the only way for this to engage the adjacent possible of someone else's research is for you to share it. Some time in the near future, you will be able to pick up your phone and speak your idea into an online service that can push this into a global science conversation. Then you can rinse the toothpaste from your mouth. "(W)e can be sure that accidents will continue to happen and, with human minds better prepared than ever before, we can expect these accidents to be turned into discoveries, marvelous beyond our imagination, through serendipity" (Roberts 1989).

Great ideas can happen any time, anywhere. And none of them were recognized as "great" at the time. Histories of science record numerous events where ideas that informed major scientific breakthroughs began as simple thoughts that occurred on buses, in showers, while walking or waking, and, most regularly, during informal conversations away from the laboratory (See: Copeland 2019; Roberts 1989). Discoveries made by mistake are commonly referred to as occasions of scientific serendipity: looking for $\mathrm{X}$ in the lab, and finding Y instead. "Serendipity is built out of happy accidents, to be sure, but what makes them happy is the fact that the discovery you've made is meaningful to you. It completes a hunch, or opens up a door in the adjacent possible that you had overlooked" (Johnson 2011). Yaqub (2018) outlines several different types of scientific serendipity that your next idea might trigger.

2.) Almost all of ideas you produce throughout the day, you dismiss as worthless. The few you write down to remember you cherish as diamonds. And yet, while the former are of little value to you at this moment, many of the ideas you have might be insights of some significance to another scholar. These ideas are products of your own genius, created without effort, because of the enormous effort you've already made through your learning. 
Don't get proud here, there are millions of researchers with similar talents, each one focussed on their own research problems. Each one's personal variety of sagacity makes their ideas different; and this difference is the key to the new information they hold for others.

What about those ideas you cherish? Those precious nuggets you hoard like Smaug in his cave? Give them away. It's a practice of the open-science Demand Sharing economy. You let others have what is most valuable to you. This generosity encourages them to do likewise. When the smartest person in the room is the room, filling this room with the best ideas opens up floodgates to creativity and innovation. The more precious your ideas are to you, the more likely they are worth being borrowed by others. When you add these to the conversation, you are also announcing your authorship of them. They are your gift to the academy that supports your own research. Others might add new insights to your idea enlarging and expanding its value. Only it's not yours any more, it's a part of an idea commons. And don't worry. You'll have plenty of new ideas to give away.

3.) Your idea needs friends to get great. An idea on its own in your head is like a seed in a seed packet. It needs ground to grow, it needs to join into conversations. Until now, these conversations happened in your lab or at a workshop. "(M)ost important ideas emerged during regular lab meetings, where a dozen or so researchers would gather and informally present and discuss their latest work.... the ground zero of innovation was not the microscope. It was the conference table" (Johnson 2011). Today, those conversations can and, perhaps, must happen at a much larger, online, table (See: Science happens elsewhere).

\section{You may be mistaken about science idea sharing in your career}

1. You think it's a self-defeating move to openly share your ideas. However, you already can share almost all your important ideas with a win-win outcome; and,

2. Funding agencies are the least efficient organizations when it comes to gathering important ideas. That RFI you just filled out is a good example.

3. Idea farming is a fringe notion, you think. It turns out that hundreds of corporations and public government organizations are actively doing this right now.

These misconceptions will also change when a culture of demand-sharing open-science is implemented across the academy. Let's explore further.

\section{You are ready to share your ideas anyhow}

Right now, today, a lot of the ideas you have that are somewhat relevant to your work, you would share if this were easy enough to do. You have any number of potential solutions for a wide range of issues in your area of research; solutions you have no intention of pursuing, 
but would really like to have solved, by someone, and today, if possible. For you, these are anti-rivalrous ideas. You don't mind if someone else, or anyone else, takes them to work on. Guess what, your idea might be a catalyst for someone else's research; just the idea that leads them to a breakthrough that will make your work easier tomorrow. If there were an idea farm on the web, you could certainly spend ten minutes a day contributing smart ideas for others to work on. At the same time, you can lard the idea farm with questions you need answers to, and pull these from the mix when they show up. The small remainder of your ideas are those you and your team might want to propose to accomplish, given funding. So you tuck these away. And even when your research proposal is being evaluated, you worry that someone in that process will grab them for their own proposal (after down-grading your proposal); such is the state of the academy today. Open science sharing would allow you to give away all your ideas, and still get recognition for them. The problem isn't greed, it's the culture of the academy that needs to change.

\section{Almost all solution ideas are non-rivalrous for any one scientist}

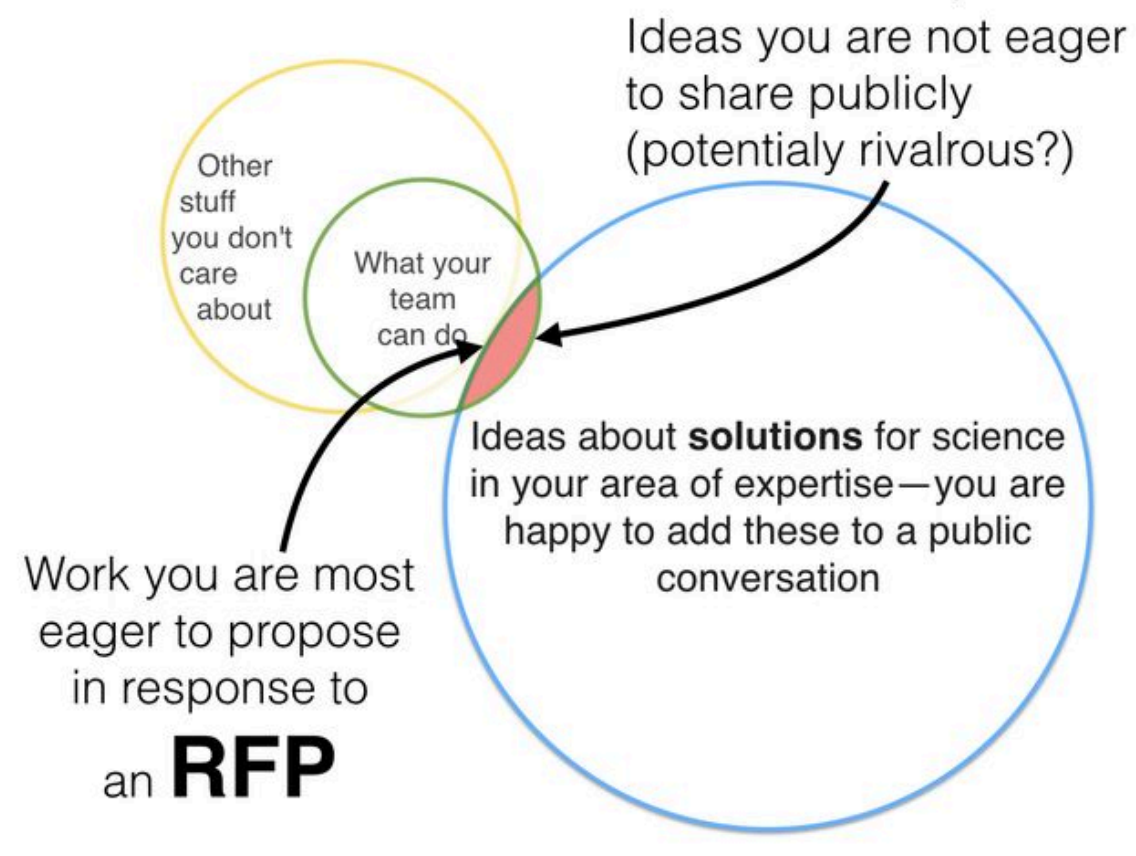

You already have no reason to not share most of your ideas

\section{The RFI paradox: scientists offer information that seeds their research}

Every so often a funding agency/foundation asks for feedback: they want your ideas about priorities for the discipline's future research. Ideally they would get a vast range of information from the thousands of scientists on their mailing lists. But realistically, they 
only get granules of ideas that are linked tightly to the goals of the teams/labs that will be angling for funding. "What should we focus on?" they ask. "Me," you answer. Not so directly, but by the content you supply.

Idea-gathering by funders is perhaps the least effective way to assemble knowledge about science. One major private foundation recently discovered, when it opened up an ideafarming platform to gather ideas that almost every idea came with a request for funding. This is not the fault of the researchers. They have five-hundred words to say what it most important for their discipline. What is most important for their discipline, in their perspective, is to support an arena of research in which the researcher has already invested.

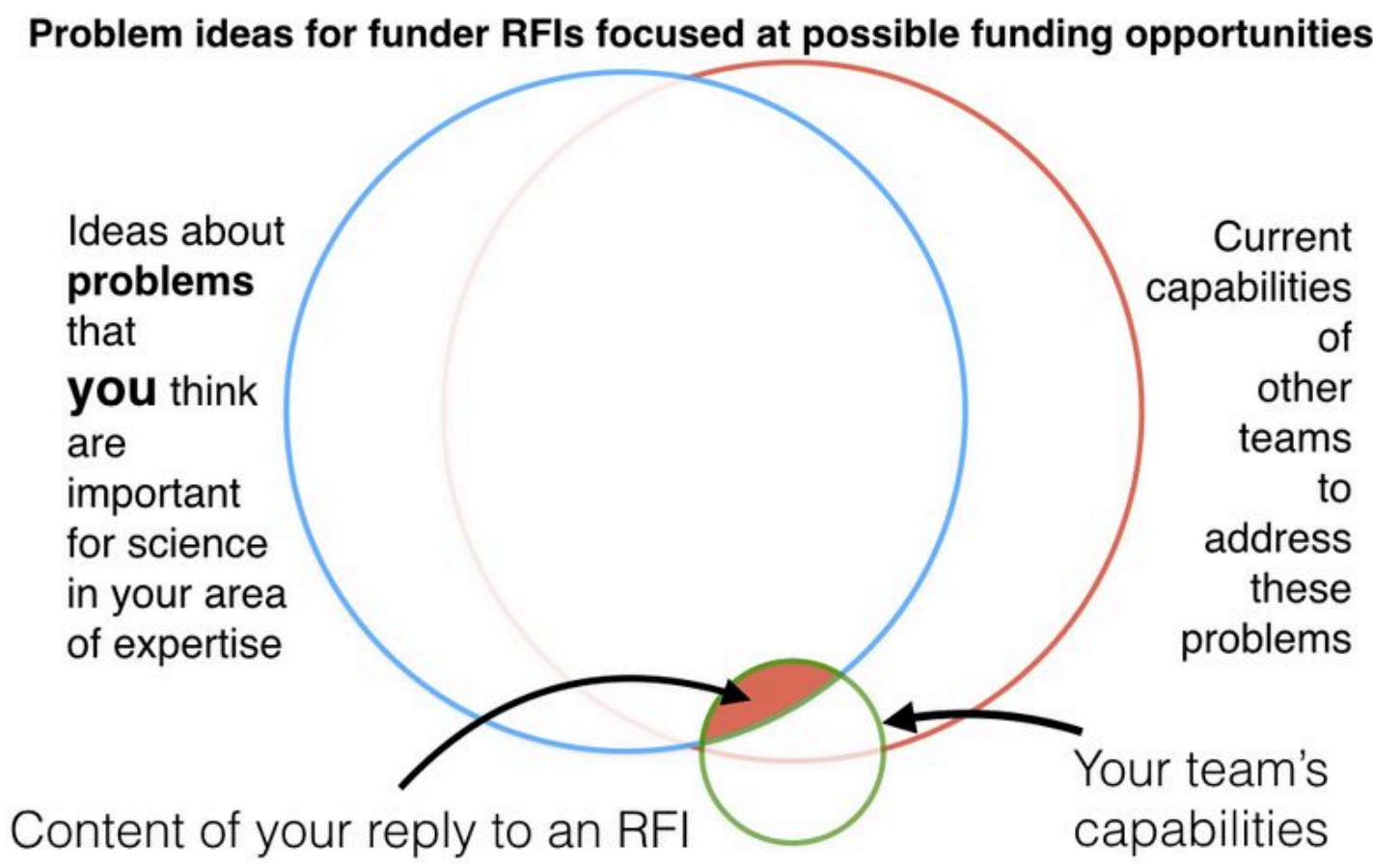

So many ideas will NOT be collected by a funder RFI

\section{An open platform for your ideas to procreate}

What if every day, say at the end of the work day, or after a beer, or in the morning after that mug of coffee, each scientist on the planet hopped online and added one idea to the global idea-farm platform (with some tags to help discovery)? What if ten-percent of them decided to add lots of ideas every month (the power-law curve suggests this is inevitable)? After a single year, there would be more than three billion ideas on the platform. Lots of overlap and similarities, but a whole lot of variety and difference too; coming from the minds of people who woke up in a hundred different nations. Each idea is time-stamped, 
with a permanent ID, and linked to its author. Every entry takes a minute or two to accomplish. A phone app lets you talk your idea into the mix.

Want to add a crazy good idea, or worried an idea might seem naive? Use your personal alias. Want to add a comment or a question to someone else's idea? Go ahead. Feeling paranoid? Lock your proposal insight into an embargoed, timestamped vault on the platform. Open this later. Then try to be less paranoid.

Demand sharing means giving what is most valuable to you to the academy. This is a value and a norm for open science. Open science initiatives are building open platforms for a variety of internet services. The platform for open idea farming may not be here now, but can be built with a bit of funding and the right home.

Link a billion ideas to a million scientists across the planet, and you can find the select few of them who happen to be considering precisely the same problematic you are puzzling through today. Then you can build collaboratives to explore these together. Thinking of writing a grant proposal? Mine the combined idea farm of the planet to make your proposal ideas better and more up-to-date; and then share these new ideas online (you can embargo them if you are worried). Your graduate students will be looking to see where their ideas are shared elsewhere, and how they can push their own infinite play into new ground. You can mine the platform to sharpen your paper or your poster. What better way to learn new things when you've already finished school, than to access the ideas of your peers? Network effects not only apply to people, but also to ideas. Put a lot of ideas into a shared, networked (databased, searchable, with discovery tools) environment, and innovation will blossom. This environment will become a place where, as Matt Ridley says, "ideas go to have sex."

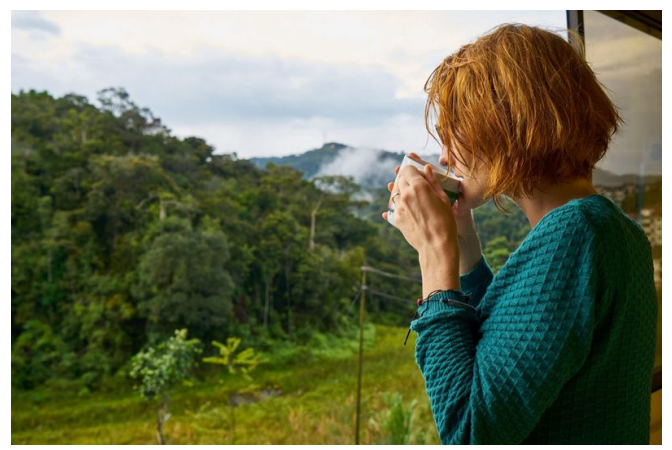

ImagiNative working on the next great idea....

\section{Become an ImagiNative}

What if one of your ideas (you had this in the shower, and spoke it into your phone app over coffee) were picked up by a lab in another county, on another continent, and used to create 
a new theory that rocked your discipline; and in the paper that announced this theory, your idea was cited as a key element? How rewarding would that be? How many times might this happen across the planet in an open-innovation environment? And what if you searched the platform and found an idea from an early-career scientist in Sri Lanka that gave you a new insight into your current work, so you cited them in your next paper. How great for them.

There is a whole lot of "elsewhere" out there in the global Republic of Science. You need to be in touch will all these elsewhere ideas and with the people thinking them who also share your disciplinary/theoretical neighborhood. As Shirky noted, "We also have to account for opportunity, ways of actually taking advantage of our ability to participate in concert where we previously consumed alone" (2010). You need to become an ImagiNative; open to new modes of collective knowing. And your lab, your school, your university needs to support open innovation (instead of patents; see: Against Patents in the Academy).

"Innovation happens everywhere, but there is simply more elsewhere than here. Silly as it sounds, this is the brutal truth: Regardless of how smart, creative, and innovative you believe your organization is, there are more smart, creative, and innovative people outside your organization than inside" (Goldman and Gabriel 2005).

One of the major changes for corporate R\&D in the past twenty years is "open innovation" (Johnson 2011). This has become a clarion call for the academy too (Europäische Kommission 2016). Sharing ideas and insights on an open platform transforms the various elsewheres of the academy into new opportunities for open innovation. Dozens of "innovation management" platforms today help global corporations mine the ideas of their wide-spread workforce and their customers.

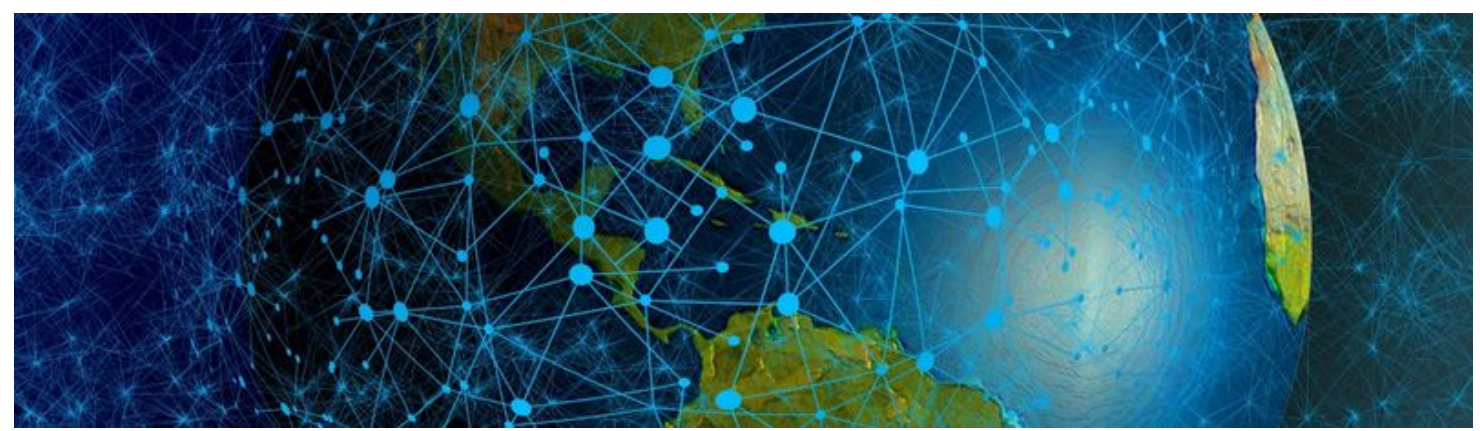

\section{Open Scientists share objects to gain network effects for their work}

"Because we have to coordinate with one another to get anything out of our shared free time and talents, using cognitive surplus isn't just about accumulating individual preferences. The culture of the various groups of users matters enormously for what they expect of one another and how they work together. The 
culture in turn will determine how much of the value that we get out of the cognitive surplus will be merely communal (enjoyed by the participants, but not of much use for society at large) and how much of it will be civic." (Shirky 2010)

Building on a civic culture of sharing, open science creates new value from every object (idea, data, method, software, results) that is openly shared. Some of this new value accrues to the scientist who shares, some goes to the benefit of all scientists working in the same research arena who reuse this object, and some goes to scientists who can open up new research from the collective resource that this object now enhances. This last value is the ultimate promise of open science: a shared surplus of research objects the can be openly mixed, mined, and melded into new, synthetic knowledge. McKiernan (et al. 2016), demonstrates the advantages of open sharing for citations, impacts, careers, etc. What the open scientist does to increase the holdings of the open corpus in their field adds a civic choice to these advantages. Growing the open research ecosystem helps every scientist on the planet.

Adding a new bit of research findings and process to an open repository is as easy (or easier) than submitting this to a closed collection (such as a for-profit publisher). However, open sharing scales better, particularly when it uses open standards-based platforms, and it is less fragile, as it can be migrated to new platforms and spread across multiple locations. Openness adds to discoverability and access, and contributes to reproducibility.

Even as the value of, say, a telephone exchange, increases with each new telephone connection, the addition of a new data set, or a null result paper, or a specific finding builds numerous interconnections with the rest of the corpus. These interconnections (and their "network effects") can lead to new knowledge, and they can serve as a mirror and a measure to reveal how each new bit of content solves (or critiques) a specific issue, and also potential problems with the newly added object. Rapid, open review opportunities arise. So too does rapid recognition and opportunities for new collaborations.

A lot of these new interconnections will take place on the internet at a planetary scale. The network effects of open science build capacity for the free movement of objects and ideas. This capacity-the almost instant global access to science products on the open web-is anathema to markets that need to claim ownership and restrict access in order to capture profits from these. Distributed data protocols (e.g., the Interplanetary File System) and other emergent technologies will reduce the cost of hosting science objects to a near zero margin. Open licenses make sharing science knowledge durable and its reuse legal.

As Cameron Neylon said at the metrics breakout of the Beyond the PDF conference some years ago, reuse is THE metric. Reuse reveals and confirms the advantage that open sharing has over current, market-based, practices. Reuse validates the work of the scientist who contributed to the research ecosystem. Reuse captures more of the inherent value of the 
original discovery and accelerates knowledge growth. Open science is a science knowledge and data reuse accelerator. Its network effects help make reuse available, and, in time, inevitable. 


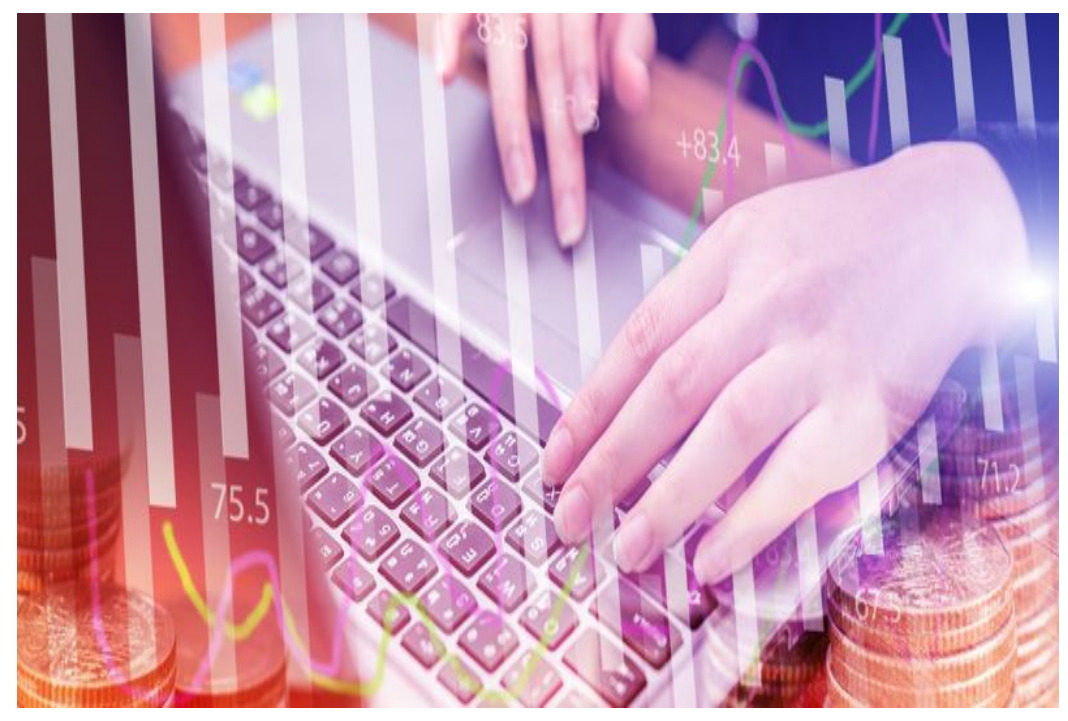

With a poor return of value, and a huge overhead for research, patents are a bad investment for the academy

\section{Against Patents in the Academy}

\section{It's time to eliminate patents in universities: Step up to Open}

"It is true that many people in science will scoff if you try to tell them about a scientific community in which ideas are treated as gifts. This has not been their experience at all. They will tell you a story about a stolen idea. So-and-so invented the famous such and such, but the man down the hall hurried out and got the patent. Or so-and-so used to discuss his research with his lab partner but then the sneaky fellow went and published the ideas without giving proper credit. He did it because he's competitive, they say, because he needed to secure his degree, because he had to publish to get tenure - and all of this is to be expected of departmentalized science in capitalist universities dominated by contractual research for industry and the military" (Hyde 2009).

In researching the forty years of allowing publicly funded primary research results to be patented in the US, what becomes clear is that for every success story there are scores of negative outcomes. The bureaucracy that universities build to capture the "value" of research as patents (Welpe et al. 2015), the administrative burden on researchers to conform their work to the process of patent-making (Stodden 2014; Graeber 2019), the perverse career pressure to produce more patents (Edwards and Roy 2017), the downstream roadblocks for sharing the research (NAS 2018): the entire ecosystem (or egosystem) of doing patents argues against their benefits to the academy. The underlying 
tension between the university's long-term mission as a wellspring of new public knowledge and the market's desire to acquire and privatize new discoveries remains at issue here (Foray and Lissoni 2010).

The Handbook is not a primary source for arguments around patents, and will only point to some major issues and a handful of available resources on this topic. Gerald Barnett (Accessed August 31, 2020) has assembled a useful resource on the web for those interested in university patent issues. The Handbook argues that open science works best within a shared resource commons, where the neoliberal market is held apart, but likely never fully absent, for the duration. Open science and closed knowledge transfer practices do not play well together.

You might work in one of very few select sub-disciplines (usually within bio-medical or IT research) at one of the few universities where university patents have had some historical financial returns. But for the other ninety-five percent of science, and for the academy as a whole, the value of sharing research far exceeds whatever near-term monetized return might be available. Newfield (2016) summarizes the situation this way:

"The point here is not that the University of California and American research were doing badly. To the contrary, they were producing the normal market results of doing research very well, which (with rare exceptions) is to spend lots of money rather than to earn it. The market results of innovative research are, as research results, close to nil. This is as it should be. The purpose of innovative research is innovation-discovery, invention, and scientific progress. This research has great long-term and social value that could not be captured as licensing revenue or estimates of the market value of patents.

The contribution of the research university can best be appreciated in broader, postmarket terms. The research university was designed to investigate every topic of conceivable public interest, from astronomical physics to agricultural genetics and everything in between. Major commercial returns accrued to research in a fairly narrow band of fields largely found in information technology and biomedicine...." 


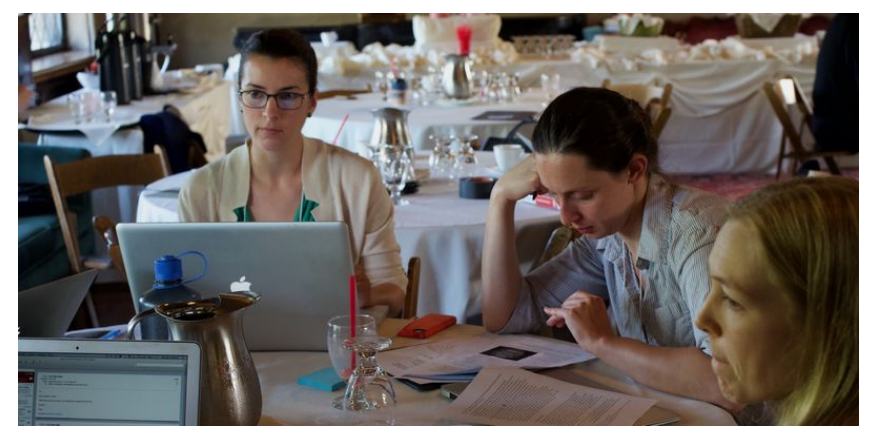

Workshops only work when research ideas can be openly shared

University research results have also, historically, been "transferred" to the academy, industry, and the public through a diverse portfolio of channels (publications, workshops, conferences, etc.). Patents interfere with these other channels. "[W]idespread patenting and restrictive licensing terms may in some cases hamper, rather than promote, technology transfer from universities to industry. These policies may also obstruct the process of scientific research (Mowery et al. 2001). Foray (2004) puts it like this: "Most studies on these issues show that this evolution [toward patenting basic research] represents a real risk of irremediable alteration of modes of cooperation and sharing of knowledge in the domain of basic research. When there is nothing left but exclusive bilateral contracts between university laboratories and firms, there are forms of quasi-integration that undermine the domain of open knowledge."

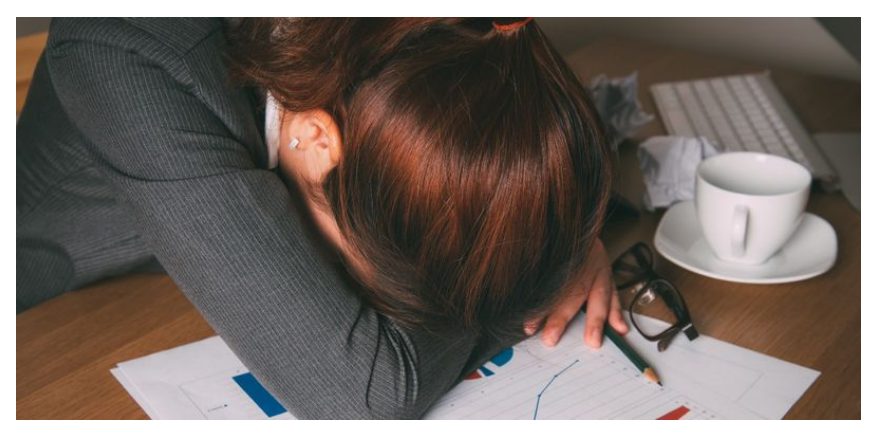

The number of university patents has exploded in recent decades. Patents have become a gameable metric; another administrative burden that takes time away from doing research.

Remembering here that science is infinite play (See: Learning infinite science play). The actual returns on research are mostly "postmarket" in value. Open sharing accelerates returns in the near term and compounds research value over time. Universities achieve 
their value proposition through a broad range of research and educational activities. The availability of market returns from patents for a small segment of university research threatens to warp the research opportunity landscape, and the normative internal incentives (including curiosity) for research (Strandberg 2005).

"In an age when ideas are central to the economy, universities will inevitably play a role in fostering growth. But should we allow commercial forces to determine the university's educational mission and academic ideals? In higher education today corporations not only sponsor a growing amount of research - they frequently dictate the terms under which it is conducted. Professors, their image as unbiased truth-seekers notwithstanding, often own stock in the companies that fund their work. And universities themselves are exhibiting a markedly more commercial bent. Most now operate technology-licensing offices to manage their patent portfolios, often guarding their intellectual property as aggressively as any business would. Schools with limited budgets are pouring money into commercially oriented fields of research, while downsizing humanities departments and curbing expenditures on teaching" (Press and Washburn 2000; Accessed August 25, 2020).

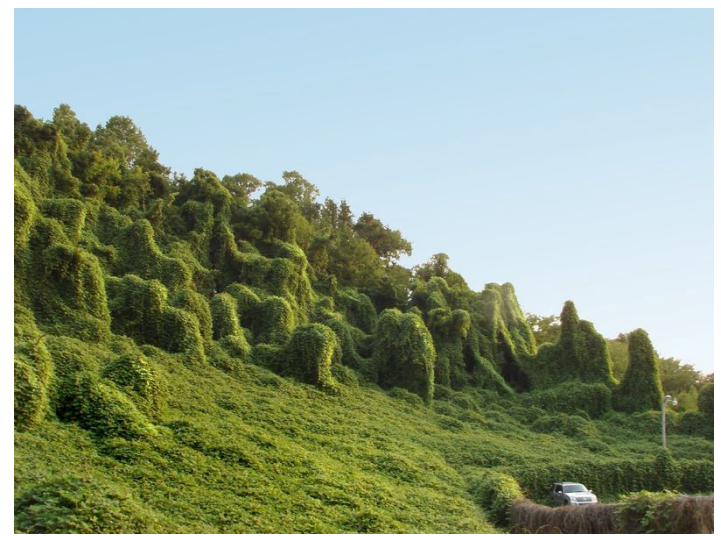

University patents grow like intellectual property kudzu. They choke once-promising research pathways and deter new research as they cover the innovation opportunity landscape. (Photo: Katie Ashdown [CC BY 2.0]/Flickr]

\section{Time to act}

Open science looks ahead to a future where the capacity to share research findings is optimized through scholarly commons, collaboratives that steward research goods through the decades, and across the planet (See: scholarly commons; Also, Madison et al. 2009). Patents subtract intellectual property and value from these commons: "[T]o the extent that universities surround the work of their scientists with thickets of patents, the upshot can be 
what Heller and Eisenberg [1998] call a scientific 'anticommons' in which ideas and concepts that in the public domain might spur discovery and innovation are zealously guarded by the institutional owners who value income more than innovation" (Ginsberg 2011). Researchers may also shy away from research arenas where existing patents impede new research (Foray and Lissoni 2010).

Looking ahead, the rapid increase of mostly under-performing (in terms of financial gain) patents creates no-research zones across formerly attractive knowledge domains. This growing patent infestation - intellectual property kudzu clogging the shared open resource pool - may be an unfortunate near-future end game for university patents, strangling new research. But a better plan is to clear away these anticommons today.

In the US, the repeal of Bayh-Dole- the act that permitted universities to patent federallyfunded research - would open up old (and now, new), long-term research sharing capacities (Barnett, May 10, 2020; Accessed August 26, 2020). Putting the market-incentive genie back in its bottle will help universities shrink their administrative overhead, help researchers manage their own research interests, and help the academy get on with the real business of science: its mission to openly share knowledge within an abundant gift economy in order to foster new discoveries of benefit to all humans. However, Bayh-Dole is only one of a couple dozen post-WWII US laws that regulate and channel intellectual property flows among universities, government labs, and industry (Slaughter and Rhoades 2010). These laws were created to knit university research outputs into the surrounding neoliberal marketplace. Each of these laws needs to be reassessed for its impact on the other knowledge dissemination flows universities have long used, and on the long-term mission of academic organizations. As the U.S. Code is a maze of regulations that are stacked on previous laws, simply repealing one of these (such as Bayh-Dole) is rarely a good fix. Its removal simply exposes the problems created by the previous laws (Barnett, August 31, 2020; Accessed September 1, 2020).

There are two options around Bayh-Dole. The first would be a new national law that revokes and replaces large parts of Bayh-Dole without repealing it; a kind of Bayh-Dole antidote that neutralizes the previous law and adds another wart on the dimpled surface of the U.S. Code. The problem here, as John Wilbanks (personal communication) surmises: universities would likely find contractual means to work around the new law and keep doing what they do in a somewhat weaker mode. A university culture of neoliberal, shortterm gain will find a way to circumvent the new law. The second option is more pervasive and effective over time: change university culture to neutralize Bayh-Dole. "Any university could in effect repeal Bayh-Dole by creating an open scholarship favorable patent policy. Claim nothing up front. Require no disclosure of inventions" (Barnett, ibid). Here is a concrete cultural change that open scientists can take to their faculty senates and board of regents. Barnett (ibid) spells it out with some precision: 
"Thus, the shortest route to open is to insist that universities comply with the extraneous requirement of the nonprofit standard patent rights clause at $37 \mathrm{CFR}$ 401.14(f)(2)-require the written agreement, making inventors parties to each funding agreement, and declining to take any interest in any invention the inventors might make under the funding agreement (which in turn brings the university into compliance with the extraneous requirement at 37 CFR 401.14(g) (1)). With compliant (f)(2) agreements in place, inventors have no obligation to disclose subject inventions to the university or to the federal government so long as the inventors do not make the inventions know[n] to the inventors' patent personnel and the university does not claim ownership of the inventions and require the inventors to make the inventions known to the university's patent personnel."

Need more detail? Barnett (September 8, 2020; Accessed September 9, 2020) gets to the heart of your new university policy. If your mission is to seed new knowledge to the world, your university can do this a lot better without exclusive patents.

Once the university's culture has pivoted to open, technology transfer offices (downsized appropriately) could play a part in encouraging open and free licensing agreements that seed new knowledge out to the public. "Universities have, for a very long time, seen themselves primarily as dedicated to the advancement of knowledge and human welfare through basic research, reasoned inquiry, and education. The long-standing social traditions of science have always stood apart from market incentives and orientations. The problem is therefore one of reawakening slightly dormant cultural norms and understandings, rather than creating new ones in the teeth of long-standing contrary traditions" (Benkler 2006).

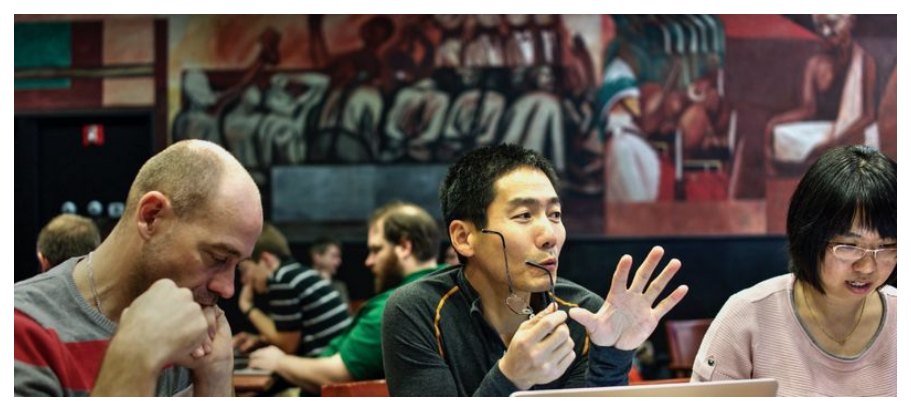

Research ideas are conversation starters. Openly discussed, they foster creative moments of serendipity. Patents are conversation enders. They lead to silence.

Research ideas are conversation starters. Openly discussed, they foster creative moments of serendipity. Patents are conversation enders. They lead to silence. 


\section{Life after patents}

There are some universities, and hundreds of active academics - and associate vice chancellors and assistant deans - who have benefited financially (or defended their job salaries) from university-patent-driven technology-transfer practices enabled by these laws. There is an argument that universities need this new source of funding in the face of other budget cuts; that universities should realize immediate returns of the value of their research. However, such an argument already discounts other, and greater, value that open research might provide in the absence of patents. The larger corrective to current budget issues begins with a more complete understanding of the sum of the value of the public goods created by universities.

Newfield (2016) details the path to more fully optimize the value proposition for universities:

"We saw that the road to the public university's decline was paved with a long, diffuse campaign against its status as a public good. The practical effects were disastrous. The demotion of public good status forced university managers to pare their institutions' overall value to a narrow and fragile private fraction of the total (the wage premium over high school graduation). This paring undermined the university's ability to deliver the indirect, nonmarket, and social benefits that make up the majority of its total value, and its ability to deliver the emerging private market good, which were creative capabilities, which paradoxically could not be supported by private good market calculations. The failure to make a strong case for both individual and mass creativity, which depended on rebuilt support for research as well as instruction, weakened the case for rebuilt public funding. Collateral damage includes weaker understanding of the public value of academic freedom for faculty, of due-process-based job security for all university employees, and of the need to convert student work time to study time.

The solution requires restoring the university's public good status. A first step would be basic accounting reform that quantifies the value of indirect effects, nonmarket value, and social benefits with the same dutiful attentiveness that accounting applies to the private market benefit of higher salaries" (Newfield 2016).

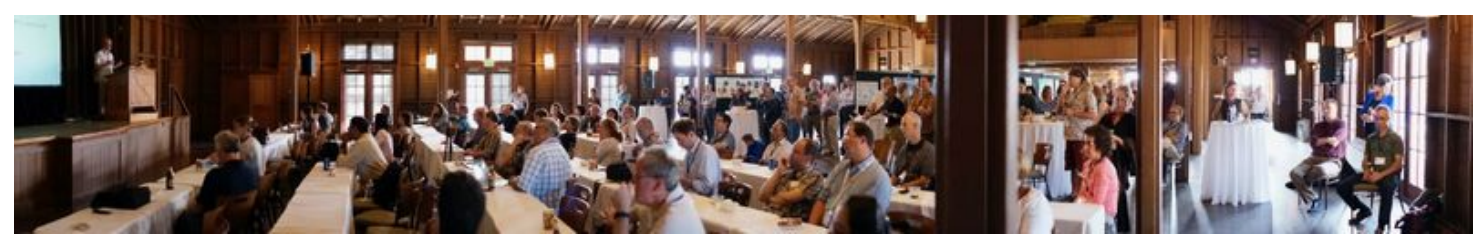




\section{Open science promotes multiple pathways for sharing new knowledge.}

Open science looks to build on the longer-term historical/future mission of the academy as a wellspring for creative outcomes, both research and learning. All through this Handbook, you can find information and explanations on how open science cultural changes - many of which merely revitalize lost cultural norms - build innovation capacity and internal incentives that can drive science forward. Because of the compounding feature of open research collaborations, open science is likely to also improve the direct financial return on research within society, even though universities do not capture this return through patents.

Newfield (2016) proposes a multi-stage recovery from the neoliberal university. As open science works inside the academy to optimize the complete value of doing scholarship, this added value can become the subject of an active conversation with government funders and legislatures, who will be tasked to reinvest in the higher education endeavor as a public good with a solid value proposition.

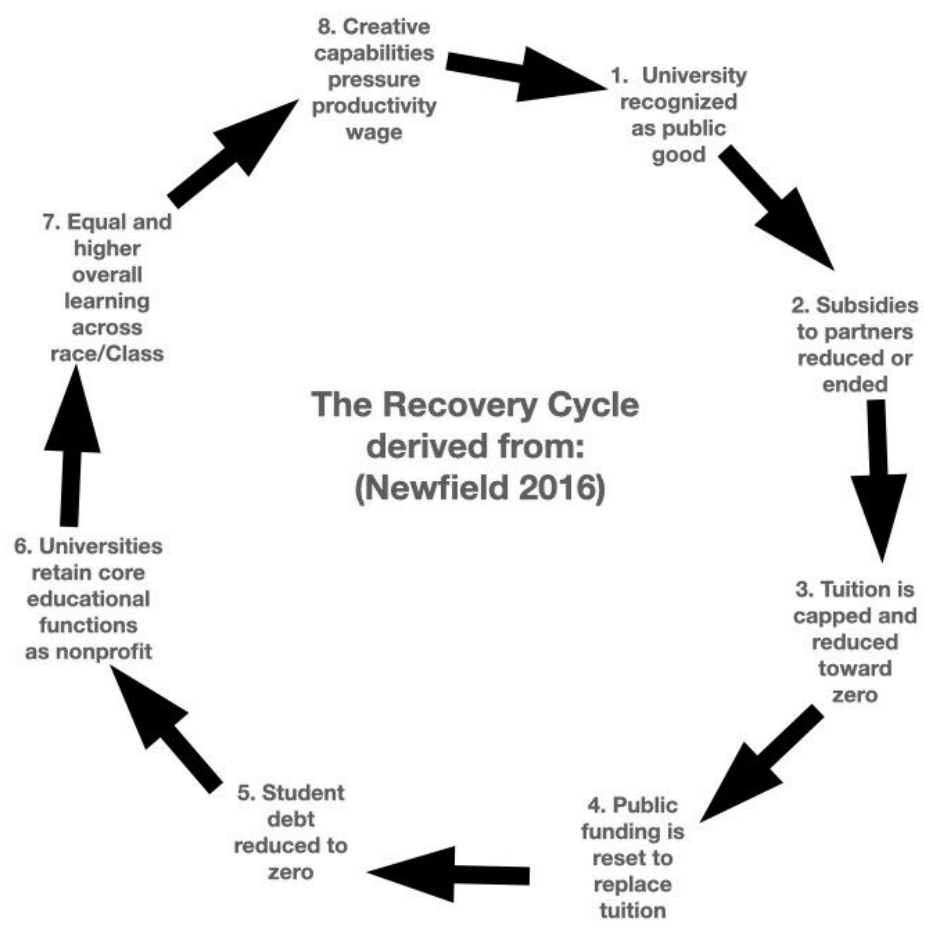

Recovery from the private-goods-only evaluation of the university value proposition begins with a new appreciation of the larger creative capacity universities offer society. Open science harnesses this capacity, making it more available to public notice and, one hopes, support. 


\section{Your take on this}

You can help your university become more open by working toward a post-patent culture. What is your sense, from your own experience working with and around university-held patents? How much of your time is spent dealing with demands for patentable technology transfer? Has the presence of existing patents caused you to shift your research topic? The future of patents is one more conversation within the cultural shift toward open science. Your ideas are valuable, perhaps these should be gifted to the academy (See: Idea Gardening). 


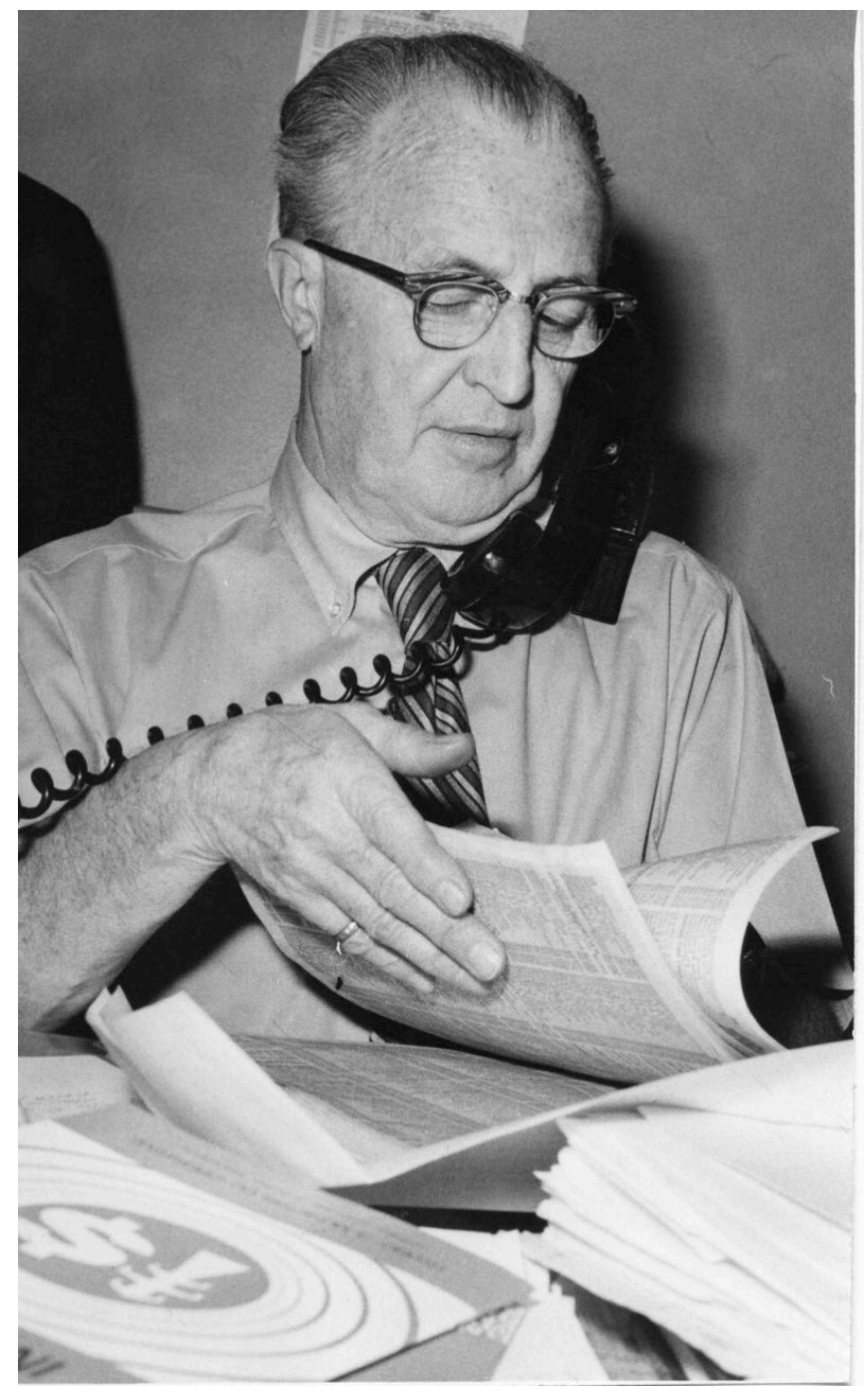

Remember telephone books?

"[M]any people, especially those in positions of influence, strive to 'do things better,' which in practice amounts to 'do obsolete things better'"(Dindersmith 2018).

\section{Is My Learned Society Obsolete?}

This is just one question any open scientist hired on staff, or volunteering as a leader at a learned society needs to ask herself, and then others. In the last century and before that, learned societies flourished as homes for sub-disciplinary (and sub-sub-disciplinary) journals and annual conversations. They also provide a lobbying voice for their segment of 
the science endeavor, which itself may be obsolescent. They offer a place for new scholars to meet established scholars, and they recognize and reward exemplary work.

Michelle Brook <https://quantumplations.org/> did a count of learned societies in the UK, and found more than eight-hundred of these. Across the globe there are thousands. One can imagine that each one of these does some good within its purview. The other side of this good, however, is the opportunity cost of their work (members might get more value elsewhere), and their relative ability to contribute to and benefit from open science. Your learned society may not have changed much for decades. Remember telephone books (and "telephones")? They were once essential. Now they are landfill. In the face of opportunities and change, all learned societies will need to find nimble footing going forward:

“[L]earned societies are part of the UK's knowledge economy and they can expect to see the pace of change and external competition increasing, so having a forward-thinking, adaptable and change-welcoming culture is important to their future survival" (Gardner 2013).

If your learned society is, in part, or on the main, obsolete, then making these obsolete bits better is the wrong way to spend your energies. Instead, you need to start the process of replacing obsolete practices, behaviors, and attitudes. This will be hard, but there are good resources here in the Handbook to help you out. So, what does obsolescence look like in the academy?

Note: there are lots of other (organizational, attitudinal, etc.) ways that your society may be obsolete. Here we will just list some of the ones that impact its role in open science.

\section{Seven obsolete features still found in learned societies:}

1. Journals/monographs that are based on the production of a paper product, and distributed through a subscription model.

The form of a paper journal creates an arbitrary scarcity to the publication. It's like you are publishing and privatizing the work of your members at the same time. University libraries are cajoled into subscriptions that must then be renewed for continuity (Also See: Learned Societies, Open Access, and Budgetary Cross-Subsidy <https://eve.gd/2019/09/17/learnedsocieties-open-access-and-budgetary-cross-subsidy/> Accessed September 17, 2019). Members are tasked to provide peer review. Most likely you already do a digital version, anyhow.

1. Step away from the cellulose and subscriptions and use the Internet. It's been around for decades. Your scientist readers around the globe will approve. Do you pay for what you do (outside of publishing/privatizing the journal) with moneys from subscriptions? First, you've been charging too much. Second, if what you do 
(scholarships, prizes, lobbying) is valuable to your members then charge them a membership fee. There are new business plan ideas that can help wean your society from its subscription addiction. Check out Harvard's Societies and Open Access Research (Accessed June 24, 2019). More than a thousand societies (Accessed August $2,2019)$ already have switched to open access. Add your name to this list! Suggested citation here: Society Open Access Research (SOAR) Catalog. Suber, Peter; Sutton, Caroline; and Page, Amanda. January 2019. http://bit.ly/oaj-society. CC-BY-SA 4.0 International

\section{Conferences with more than 300 participants.}

This is not to say that you should not offer co-present events. If you are running a conference with a couple thousand members (or more, or many more), you are complexifying the central reason people have flown into your meeting: to find each other, and make new, or revive old, personal associations. Stepping into a big-city convention center with your colleagues may feel good, for about the first hour. After that it's all random noise.

Your conference carbon footprint is inexcusable. You should respect and support members who pledge to not fly at all (<https://www.theguardian.com/science/2019/jun/29/noflights-four-day-week-climate-scientists-home-save-planet> Accessed June 28, 2019)(See also: should climate scientists fly Accessed June 24, 2019). Find creative ways to split up large national/international meetings into a number of smaller meetings with better focus and a lot more interpersonal time.

\section{Poster sessions with no digital archive.}

Posters are a good way to show off work in progress, and an opportunity for small-group interactions. They do take a significant amount of time to produce, so they deserve a permanent home on the internet. If the way you do your poster session is obsolete, you can fix that. Find an open online platform for poster sharing and use it well. And use better poster design requirements (Accessed June 24, 2019; video here) to help start conversations.

4. Meetings dominated by plenary talks and sessions devoted to individuals presenting papers.

Apart from a few plenary talks curated to help the room consider new technologies and findings that stimulate conversations, most talks can be recorded and posted online before the meeting, and not take up the agenda. Instead, do workshops and panels that provoke discussions and learning moments. Use conversation models (such as the world cafe) to bring together early and late career professionals. Long breaks, good food, nearby coffee houses, and beer also help. 


\section{No support for member collectives/clubs.}

A mailing list is not a "community." You need to broadcast less and listen more. Your members need to find others working in very similar research arenas, and to have peer-topeer online collaboration tools. Help them do this. Become research match-makers and coordination masters. That's your new value proposition.

Are your association's membership numbers a metric for you? Is bigger really better? Remember that the $r$ /science sub-reddit $<$ https://www.reddit.com/r/science/> has twenty million members. Theirs is bigger than yours. Go online and build active communication/ collaboration resources for your members.

\section{No support for cross-disciplinary assemblages.}

Real innovation happens when people bridge between disciplinary/domain-specific silos. The least your society can do is open up its digital holdings to be indexed broadly by others. The clubs that your society supports need to find clubs in other societies to share their issues and problems. You can seek out venues and avenues for conversations (online or in person) with other societies. No society is alone in this.

7. Perhaps your whole learned society is obsolete. You may need to visit the "realm of chaos," (See: The Work of Culture) perhaps at a board retreat somewhere, and rethink the entire purpose and vision for your society. If this society was created in a previous century to support a then-new sub-disciplinary journal, it may be a good time to pass this responsibility over to a pre-print repository and go home. Got some endowment money left over? Do a final meeting, make it free for graduate students and early-career folks, and challenge them to come up with the next best thing. Roll over the endowment to that. Got a big, fancy building for yourself? (Good for you!) Put it on the market, and use the funds to help all your employees find good jobs elsewhere.

"If you dislike change, you're going to dislike irrelevance even more" (Eric Shinseki < wikiquotes $>$ Accessed 12/28/19).

\section{Don't stay obsolete, be leaders for the future of your domain}

Learned societies are well positioned to take leading roles in changing the behaviors of their members, though inclusive, member-led, reflexive culture change activities. They are often, however, keepers of a type of history that needs to be severed for them to move ahead. Their ceremonies and honors point to the past, to a graveyard of internally honored individuals (mostly white and male). They would do well to supplement their current honors with new ones that celebrate the future of their fields: early-career members, emerging sub-domains, collaboration successes, sharing and reuse. 


\section{Open Science Badges are Coming-and the New Nobel}

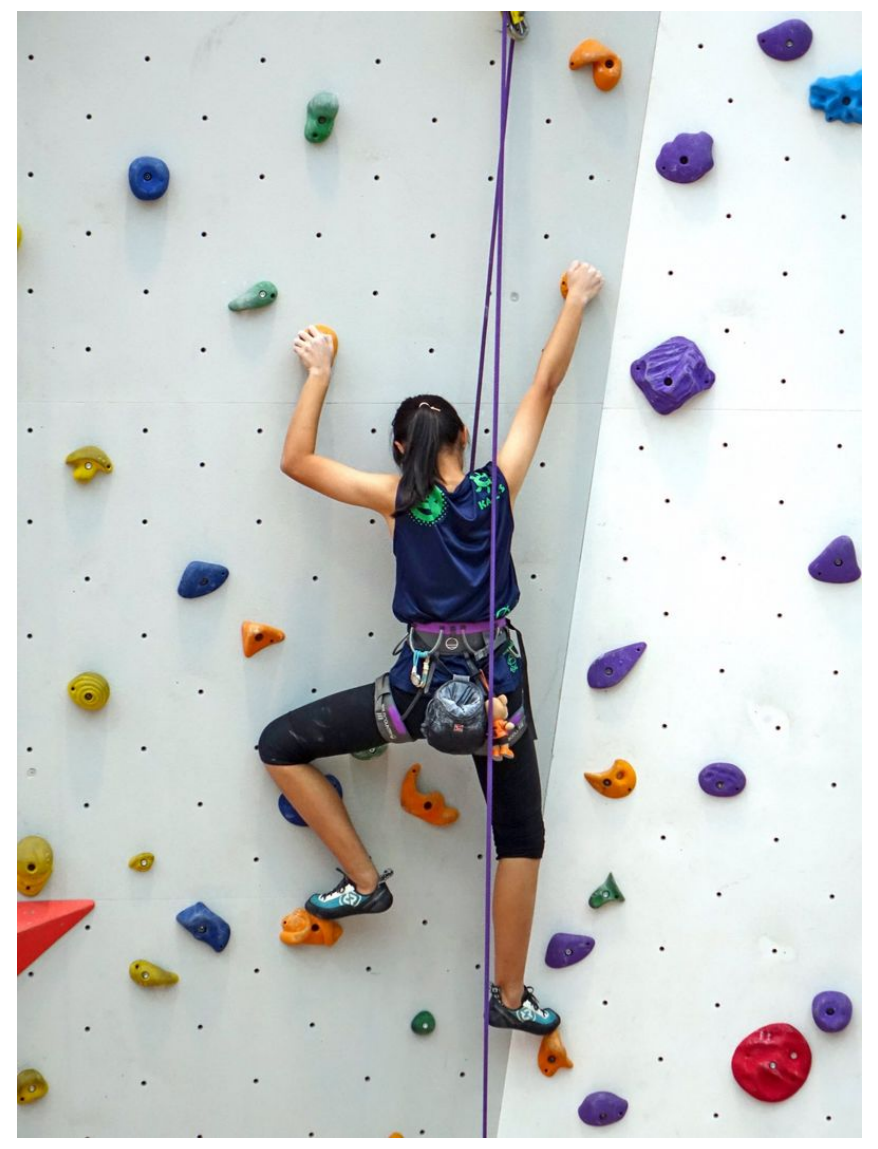

This paper outlines and addresses a working set of definitions, ideas and guidelines around the use of digital badges within connected learning contexts" (Mozilla and Peer 2 Peer University 2012).

The notion of using open digital badges to acknowledge certain practices and learning achievements has been circulating in the open science endeavor for more than a decade. Over these years, this has become a perennial "near future" augmentation/implementation of how open science can recognize and reward practices and skills. Instead of using gameable metrics that rank individuals as though they were in a race, badges can promote active learning, current standards, professional development, and research quality assurance.

The transition from arbitrarily scarce reputation markers (impact metrics, prizes, awards) to universally available recognition markers also helps to level the ground on which careers 
can be built across the global republic of science. Every scientist who wants to take the time and effort to earn a badge for achieving some level of, say, research-data reusability, or graduate-student mentorship, can then show off this badge to the world. Every student/ scientist who acquires a specific skill (R programming, software reusability, statistics, etc.) can add a new badge to their CV.

In education, micro certifications can augment diplomas and degrees by pointing to specific skills acquired during the course of study. A badge can signal the attainment of a prerequisite skill for taking an advanced course, say, or a capstone skill for outside employment. These badges can accumulate into suites of acknowledged skills that students can highlight for specific future occupations. Micro-level open badges can be assembled into practical certifications (Leaser 2016; Accessed August 14, 2020).

“Open Badges are a specific type of digital badge designed to promote learneragency principles. Open Badges communicate skills and achievements by providing visual symbols of accomplishments embedded with veriTable data and evidence that can be shared across the web. Open Badges empower individuals to take their learning with them - wherever they go - building a rich picture of their lifelong learning and achievements journey. Thousands of organizations across the world already issue Open Badges, from non-profits to major employers and educational institutions at all levels" < https://www.imsglobal.org/ digitalcredentials>; Accessed August 14, 2020.

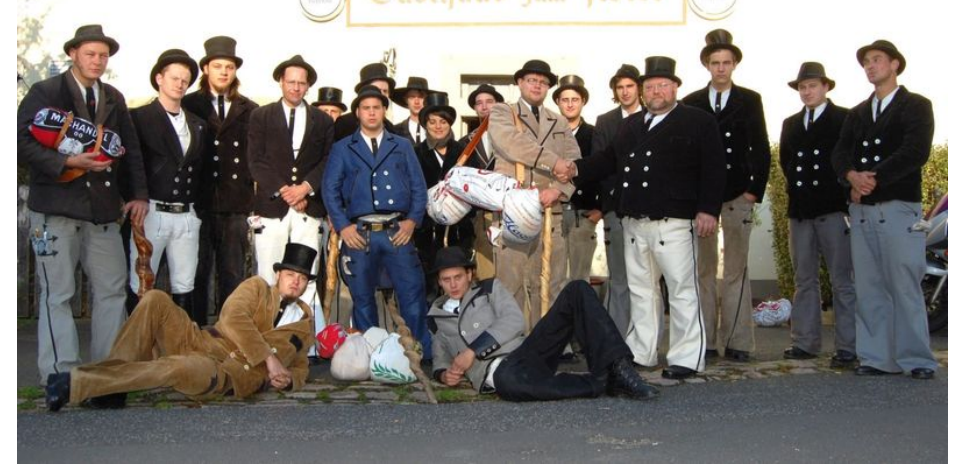

Digital badges are like virtual top hats: they signal achievement and belonging. Photo Credit: Sigismund von Dobschütz, October 16, 2011; CC BY-SA 3.0 on wikimedia commons

Above: German carpenters carry a book for certifications of their work while they apprentice on the road for three years to become members of the guild.

Keeping current with the latest badge news is difficult. Several projects are moving ahead independently (sounds like open science in general). Start-up credentialing companies, 
spin-offs from the open-badge endeavor, are building online commercial services, some of them on a blockchain, for verifiable credentials. Their not-so-open badges help companies run internal educational services and streamline hiring for specific skills (See: https:// info.badgr.com/).

You can check out open/digital badge resources on the web:

- Wikipedia: Digital Badges(<https://en.wikipedia.org/wiki/Digital badge>; Accessed August 14, 2020),

- $\quad$ MIT initially created an open standard for blockchain-connected certifications (<https://www.blockcerts.org/>; Accessed August 14, 2020),

- Wikipedia: Mozilla Open Badges: (<https://en.wikipedia.org/wiki/ Mozilla Open Badges>; Accessed August 14, 2020).

- The Mozilla effort was moved to IMS global where a standard for open badges is (currently) maintained (<https://www.imsglobal.org/activity/digital-badges>; Accessed August 14, 2020).

Of course, badges are not new. Philipp Schmidt (2017; Accessed August 17, 2020) points out a long, global history of verifiable certifications. Boy and girl scouts have used badges for a century or more. Academic diplomas are badges of learning, as are driver's licenses (in theory).

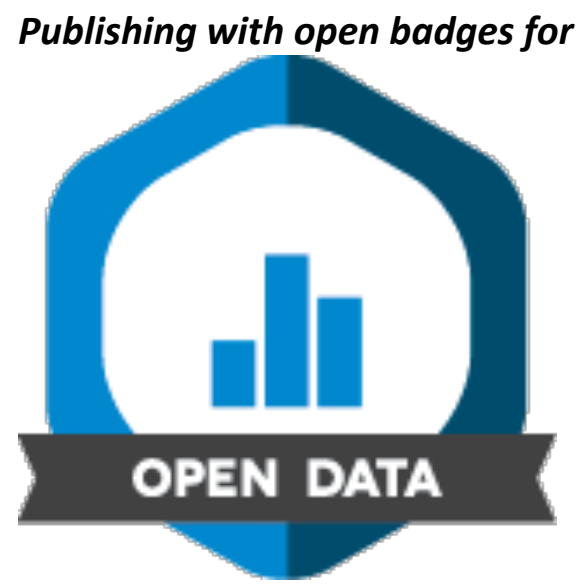

Open Data badge on the COS Open Badges Blog

One place where badges might be implemented early is in open publishing, where publishers can add badges to their online descriptions of articles. These badges would serve to mark adherence to specific open practices. "Badges are an easy means of signaling 
and incentivizing desirable behaviors. Journals can offer badges acknowledging open practices to authors who are willing and able to meet criteria to earn the badge $[(<$ https: $/ /$ osf.io/tvyxz/>; Accessed November 17, 2019)]. Badges acknowledging open practices signal that the journal values transparency, lets authors signal that they have met transparency standards for their research, and provides an immediate signal of accessible data, materials, or preregistration to readers. Badges allow adopting journals to take a lowrisk policy change toward increased transparency. Compared, for example, to measures that require data deposition as a condition of publication, badge implementation is relatively resource-lite, badges are an incremental change in journal policy, and if badges are not valued by authors, they are ignored and business continues as usual" (Kidwell et al. 2016).

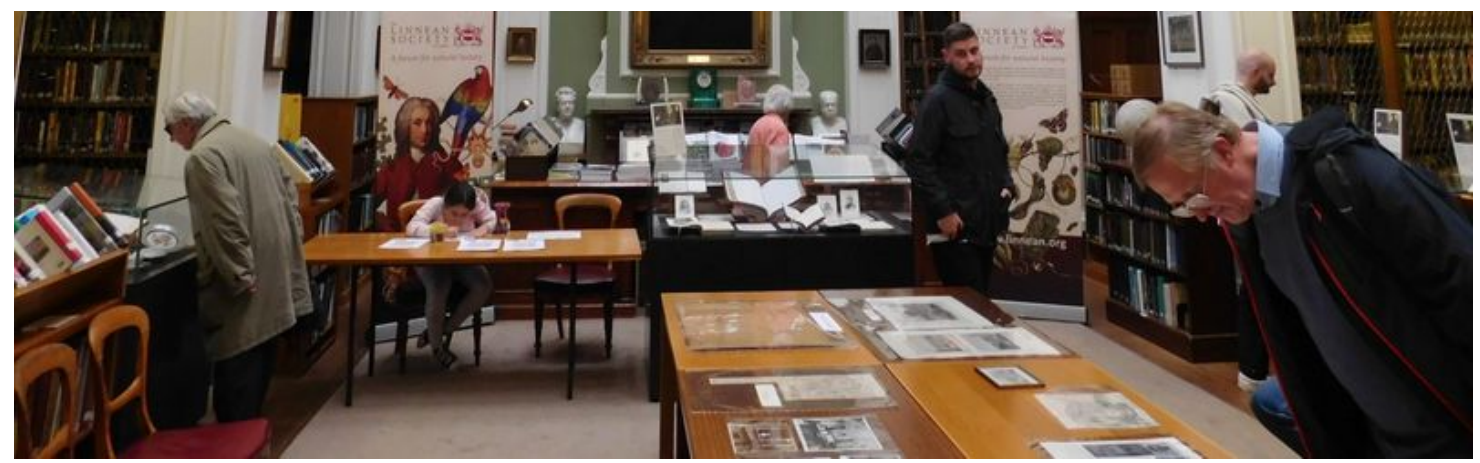

Photo Credit: Paul the Archivist on Wikimedia CC Attribution-Share Alike 4.0

\section{Learned Societies as badge engines}

Each learned society could also host badges that members can earn by sharing their research or offering services to the membership. This is an easy way to displace current journal-based reputation markers, while acknowledging quality work, and boosting membership value. Societies can reward members whose work exemplifies those norms the society determines as core to their mission. Research that demonstrates team effort, active diversity, rigorous data collection, reusability - any practice that amplifies the value of the work for the society-might be connected to a badge.

Unlike prizes, badges are open to all who meet the requirements; there are no losers here, except sloppy science. In the post-subscription business world, learned societies need to explore new value propositions. Badges are one way their communities can tap into their collective strengths to add real value to the lives of their members. At the same time, they recognize every member who qualifies, instead of awarding prizes to a selected few (See Also: Shaming the giant). How about this for a culture change practice: you acquire the right combination of badges and you automatically become a "fellow" of the society. You will have earned it; nobody needs to vote for you. 
If your learned society is not planning to offer badges, you might want to inquire about this. They are missing a golden opportunity. There should be a badge for that.

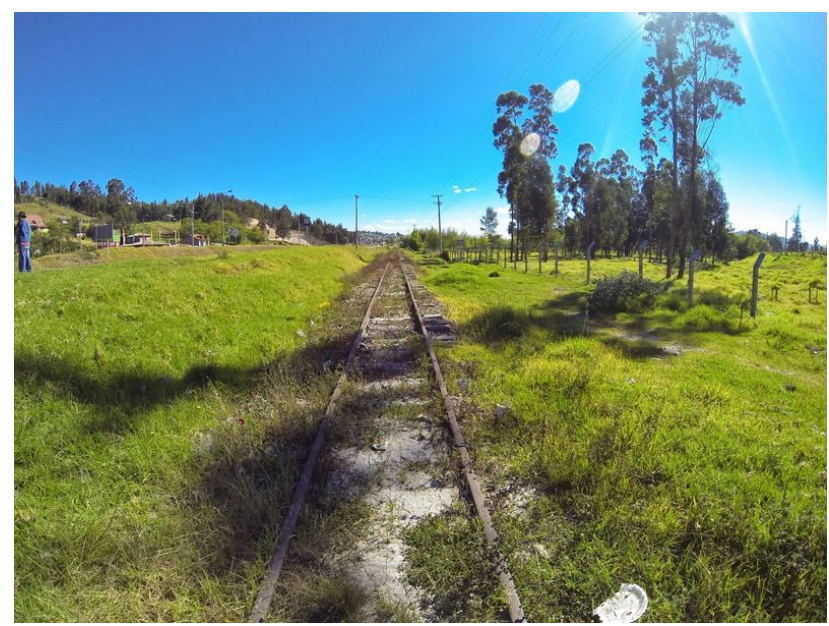

Does your old/current organizational cultural path lead to nowhere? Blaze a new path with open badges.

\section{Badges for culture building}

Badges are not easy to administer. Like all recognition schemes, they need to be well crafted and constantly tended to assure validation and verification. Badges focus attention on the practices and skills they announce. The governance of badge systems requires - as it also acquires - an active, reflexive cultural capacity to build trust and buy-in. One upside here, is that the work of supporting badges can also help an organization maintain its cultural norms over time. Badges help build communities. The conversations about badges can bring out the virtues and values of the group.

To change an organizational culture you first need to change the way things get done now. But how do you intercept current decision and work flows? How can you help the whole group unlearn toxic behaviors? Badges work to establish new paths for decisions and activities. They offer micro-rewards that nudge a community over to new practices. Badges can include learning requirements, exposing the whole community to relevant new information. Finally, earning a badge is a great occasion for a team mini-celebration. Even a skeptic with tenure can get a dose of good feelings when their team celebrates their recent achievement.

As badges celebrate cultural norms, they can help push toxic practices and other, external incentives into the margins. The badge system your academy organization creates can offer footholds up to the common goal of an open science destination; everybody can use the 
same badges to arrive at this shared future. There's still a mountain to climb to get to "open", only now there is plenty of room at the top, and a reliable path upward. Doing science right becomes easier when all the internal rewards are lined up. Buying into badges means buying out of current toxic conflicts-of-interest in the research flow (See: Building a gift economy: the dance of open science culture). It might be that open badges are the "killer app" for the future of open science!

The new Nobel: celebrating science events, their teams, and the history of discovery

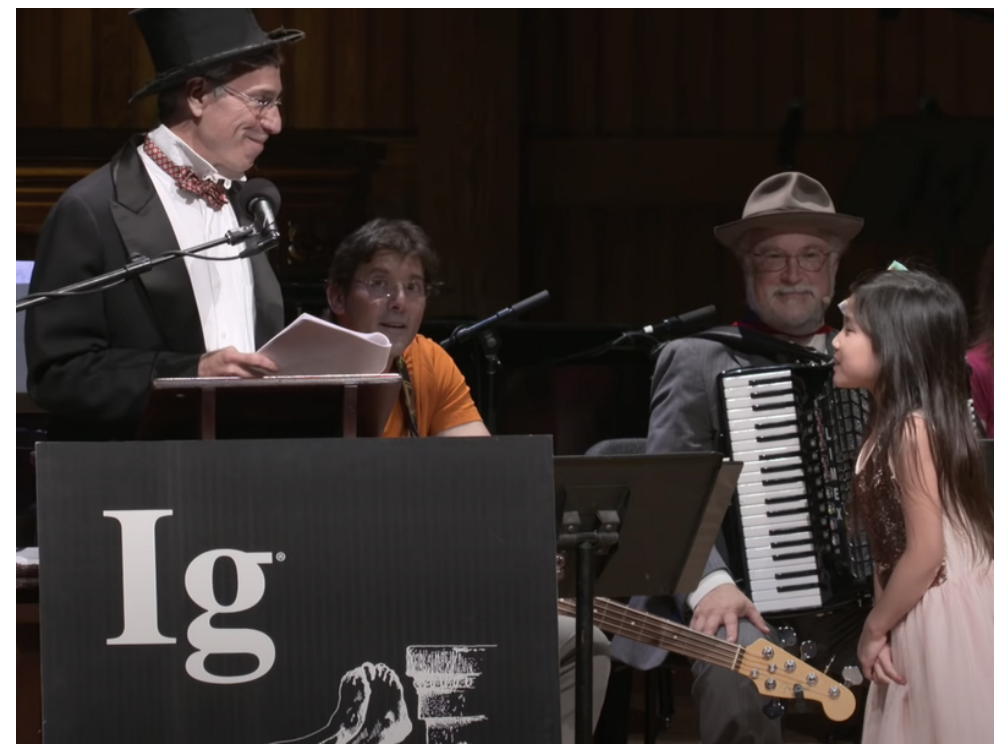

"Please stop," she yells, "I'm bored." Again and again, until the speaker relents and gives up the lectern. Time management at the Ig Nobel Prize ceremony.

“I won't have anything to do with the Nobel Prize... it's a pain in the... (LAUGHS). I don't like honors. I appreciate it [my work] for the work that I did, and for people who appreciate it, and I know there's a lot of physicists who use my work, I don't need anything else, I don't think there's any sense to anything else. I don't see that it makes any point that someone in the Swedish Academy decides that this work is noble enough to receive a prize-I've already got the prize....

The prize is the pleasure of finding the thing out, the kick in the discovery, the observation that other people use it [my work] — those are the real things, the honors are unreal to me. I don't believe in honors, it bothers me, honors bother, honors is epaulettes, honors is uniforms. My papa brought me up this way. I can't stand it, it hurts me" (Feynman et al. 2005). 
In their article, "Is the Nobel Prize Good for Science?," Arturo Casedevall and Ferric Fang review the numerous controversies linked to Nobel Prize attribution. Their conclusions are here:

"In this regard, the Nobel Prize epitomizes the winner-takes-all economics of credit allocation and distorts the history of science by personalizing discoveries that are truly made by groups of individuals. The limitation of the prize to only 3 individuals at a time when most scientific discovery is the result of collaborative and cooperative research is arguably the major cause of Nobel Prize controversies ... Changing the Nobel Prize to more fairly allocate credit would reduce the potential for controversy and directly benefit the scientific enterprise by promoting the cooperation and collaboration of scientists within a field to reduce the negative consequences of competition between individual scientists" (Casadevall and Fang 2013).

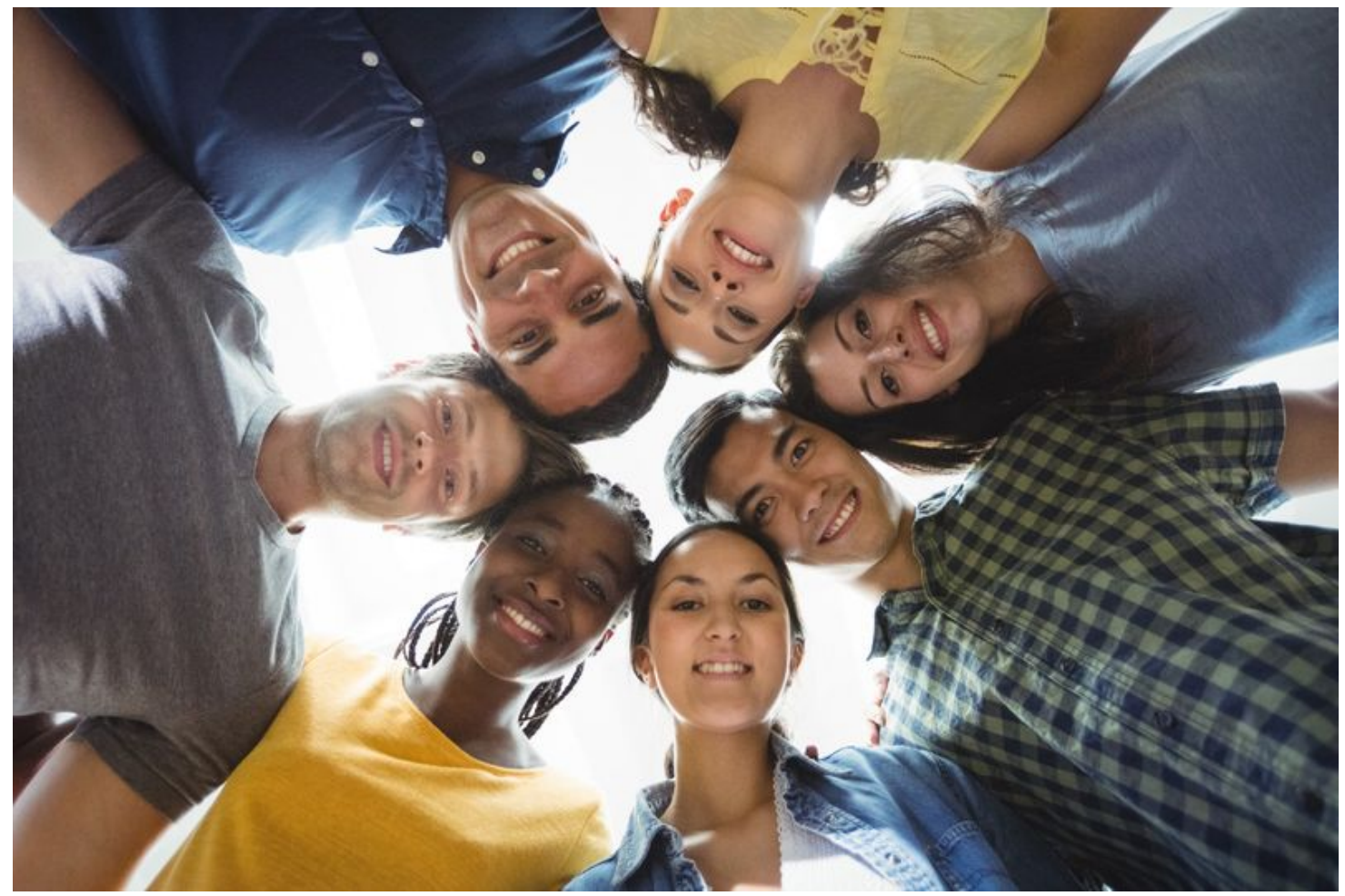

The whole team contributed. Who gets the prize?

\section{An uncommon commons populated by occasional giant ideas}

As we explored above in The Work of Culture, in open science, scientists move regularly between the complex, emergent problematics of their object of study, the complicated 
process (in research and writing) required to extract knowledge from this, and the practices of open sharing. This means that the academy commons contains a whole lot of "uncommon" artifacts, pulled with great effort from the edge of knowing.

Scientists are also uncommon, made so by the demands of their profession. While their quotidian lifestyle is mainly long hours of very hard work, they have occasional days of unusual significance: the days when the months of research pay off with new knowledge. On these special days, all the work of their team and the entire history of their domain is rewarded with a new insight, pulled from indifferent data and mountains of observation. Scientists and their teams push back against the envelope of unknowns that surrounds our understanding of the universe until these unknowns surrender new understanding. In this way, scientists and their teams create the events (Badiou and Tarby 2013) that spark giant ideas.

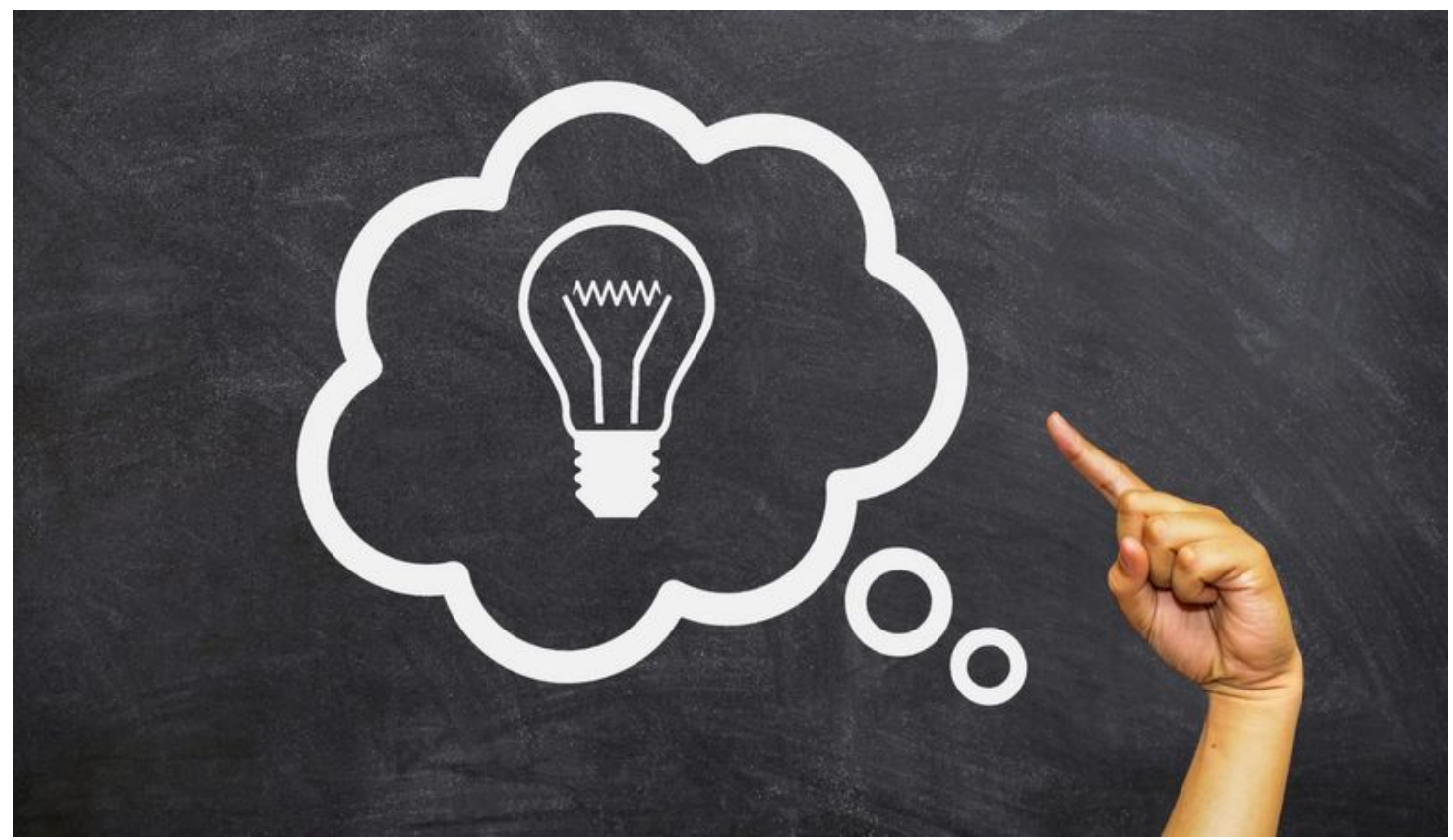

Fund new work based on giant ideas

\section{The idea gets the prize}

A giant idea, born from a moment of new knowing, perhaps in conversation, or in contemplation after conversation, or as suddenly emergent from the data, is the prize that science needs to celebrate; not the person who announces this, since the idea had been incubated by many within the larger commons. Celebrating the scientist here is like celebrating an obstetrician for having the baby, instead of for assisting in the delivery 
(“Great work, Doctor! Have you decided on a name for it yet?"). The baby, a giant new idea, birthed with some effort, might confirm and extend present knowledge with new information, or be the null result that corrects a widely held false scientific "fact," or be an insight into a new theoretical space, hitherto unspoken.

Here, the collective "mother" could be the team, the room (See below), the adjacent now, a measure of luck, and the domain's recent history. Yes, the scientist(s) here at the moment get to write up the news, but it's really the idea, this new thing, that needs to be applauded. And it is also time to give mom her due regard when celebrating the child.

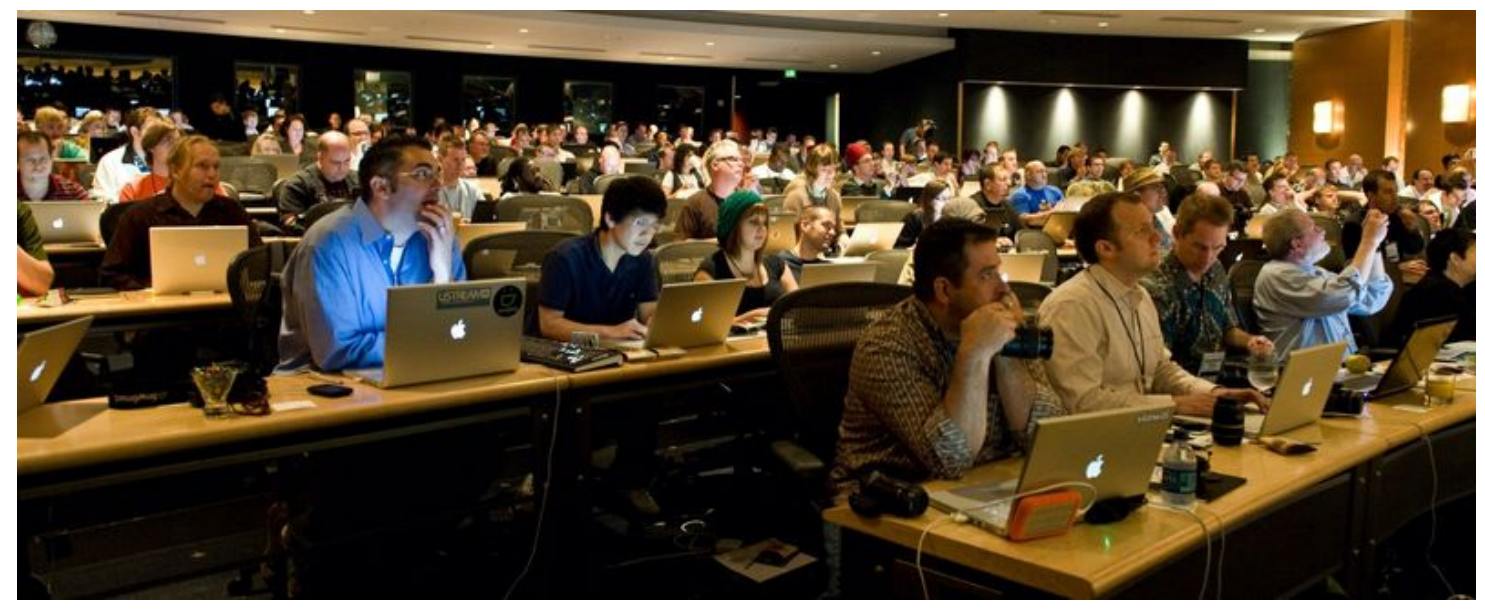

The room is smarter than any one person. Who gets the prize when a new idea is born here? PHOTO: Josh Hallett on Flickr. CCby.

"Unmooring the prize from Alfred's 'the person' bonds would happen if the physics prize were awarded to groups. This would reduce the pressure on scientists to stake their claims at the expense of others; it would offer a shortcut up the ladder of authority, a ladder some underrepresented, and thus less powerful, groups such as women and other minorities feel has already been pulled up out of reach" (Keating 2018).

\section{Science wins the award}

David Weinberger (2011) noted that "The smartest person in the room is the room itself." All the conversations in this room reflect the genius of the room, not simply that of individual occupants. Open science rewards these giant ideas by sharing them instantly, globally, and with appreciation for their value and work it took to create the event that spawned them. Open science works to spread recognition across the science endeavor, being acutely aware of cumulative advantages for some and lifetimes of research done in 
obscurity for others. The latter deserve particular attention. Science at its best is not a personal heroic quest, but open, collaborative labor.

"The Nobel Prize fits with the narcissistic vision of science peopled by heroes, many of whom are very self-centred (but who of course can turn into nice and ethical people once they have succeeded). Science requires many different skills, and it is regrettable that recognition often goes to the storytellers or the dominant males of the community. By taking into account the tacit dimension, we could also better highlight the other key roles and skills - experimenter, tool constructor, organizer of databases - that hugely contribute to the progress of science" (Lemaitre 2015).

There are a lot of people pointing at several issues around the Nobel Prize and its method of selection; you can DuckDuckGo "Is the Nobel Prize obsolete" to get a list of articles with critiques and recommendations. The Handbook adds this topic here mainly to point out how science organizations can express their appreciation for great work by focusing on the science, not the scientists.

Devang Mehta puts it this way: "Here's an even better idea: award the Nobel Prizes not to researchers but for discoveries. Imagine that today's Nobel in physics was awarded for the discovery of gravitational waves, with no list of awardees, instead of awarding it to just three scientists out of hundreds. What of the prize money? Donate it to an international science fund to promote further research in each year's prize-winning field of research. A scienceoriented Nobel (rather than a scientist-oriented one) would both educate the public in the most important scientific developments and in turn stimulate new scientific progress by using the prize money to fund the next generation of researchers" (Mehta 2017; Accessed September 12, 2020).

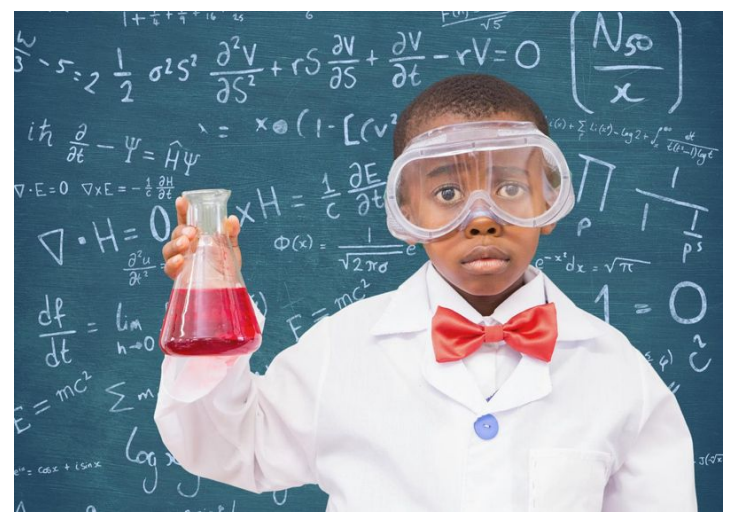

Science prizes should attract all scientists everywhere to do their best work anywhere in the world. 
The idea of giving out prizes is not itself obsolete; yet all award practices need to be refactored occasionally to capture the heart of the process of doing science, as this expands and changes in the coming decades. And, if it's time to refactor the Nobel Prize, what does that suggest for the prizes your learned society hands out? Adding an ecosystem of badges (to show off skills and accomplishments) to the recognition landscape helps to replace prizes as a central feature of open science. Since prizes celebrate brilliant work, and as celebrations as a whole add positive affect to your culture, let the prizes continue. But give them some new thought. What is your idea for Nobel 2.0? 


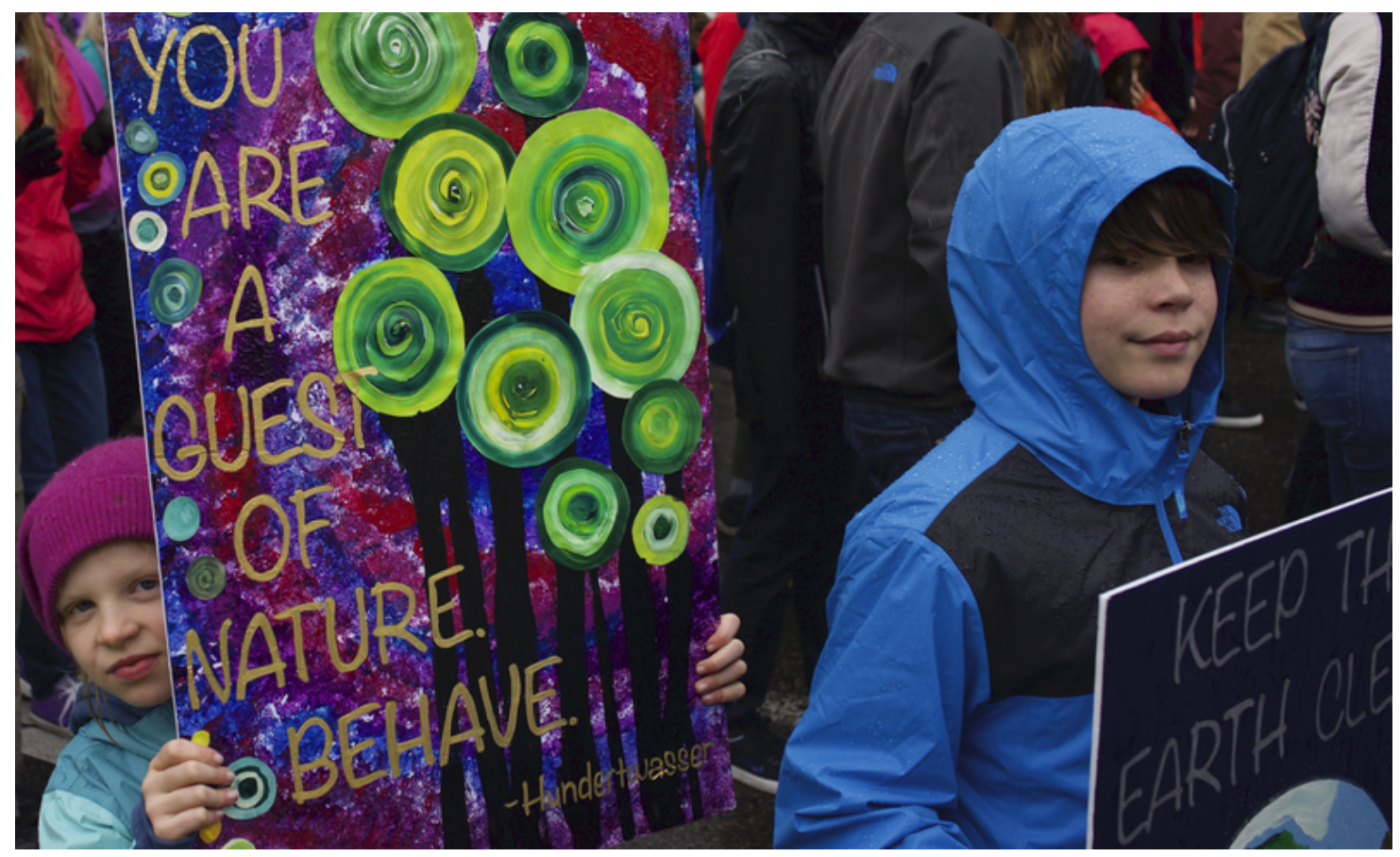

\section{The Infinite Play of Science}

"If there are at least two players, a game exists. And there are two kinds of games: finite games and infinite games.

Finite games are played by known players. They have fixed rules. And there is an agreed-upon objective that, when reached, ends the game. Football, for example, is a finite game. The players all wear uniforms and are easily identifiable. There is a set of rules, and referees are there to enforce those rules. All the players have agreed to play by those rules and they accept penalties when they break the rules. Everyone agrees that whichever team has scored more points by the end of the set time period will be declared the winner, the game will end and everyone will go home. In finite games, there is always a beginning, a middle and an end. "Infinite games, in contrast, are played by known and unknown players. There are no exact or agreed-upon rules. Though there may be conventions or laws that govern how the players conduct themselves, within those broad boundaries, the players can operate however they want. And if they choose to break with convention, they can. The manner in which each player chooses to play is entirely up to them. And they can change how they play the game at any time, for any reason.... 
Infinite games have infinite time horizons. And because there is no finish line, no practical end to the game, there is no such thing as 'winning' an infinite game"

(Sinek 2019).

In Finite and Infinite Games, James Carse (1987) makes a number of statements about culture and nature, and about human endeavors that include a wide range (perhaps very wide) of everyday human situations.

Here we attempt to insert science as an endeavor into the scheme Carse outlined, with the purpose of grounding the norms of science-however these are described-within science's infinite play with nature. Of course any scientist-as a biological organism—plays in nature at the same time she plays with nature. To play with nature as a profession is a privilege scientists all share. The finite games of finance or technology might bring in more (perhaps a lot more) money, but the struggle with universal unknowns has its own rewards. Science play, as we will see, also ties in complexity theories, emergent systems, explanation and narrative.

Today, science is an endeavor housed in organizations where we find game logics that are mainly finite (When is the next RFP coming out? What's your H-Score?). This circumstance is in direct conflict with research needs that must—-this is the main assertion here-include and support playing science.

\section{Norms point to an infinite science play}

The notion of the infinite play of science may seem foreign to scientists coached to win finite games to secure their careers. And yet all attempts to capture the normative culture of science hint at an underlying, non-finite home for science. What we find today is an academy trapped in the contradictions between these two mindsets: the poetry of discovery, the awe of nature, the joy of intellection, and the satisfactions of mentoring have been pushed aside, displaced by the rush for reputation in a now-harshly-competitive system of scarce resources and narrow opportunities.

These contradictions have been noted for decades in articles and books that contrast science's putative norms with the observable organizational practices of science. Sociologists and critics of science practice point to the realities of doing science in today's world. "Science claims X, but in practice we find Y." Ziman (2002) makes this contrast more than seventy times. These observations now share the discourse with a chorus of observations about "bad science:" unreproducible findings, plagiarized and repetitive science articles, ersatz statistics (p-hacking, etc.), systemic biases and conflicts of interest in funding and advancement, public distrust of science findings, and a profiteering publishing industry. 
The reality of doing science today seems fundamentally out of step with how good science needs to happen. "Real science" is still distinguished by normative behaviors and values that are regularly called upon to counter deviation into "bad science" (See: Zimring 2019; Accessed November 8, 2019). But when the incentives are perverse and pervasive, resistance is a challenge that overlays and undermines the challenges of doing infinitegame science. So, what happens when the reality of being a scientist fails to support "real science"?

\section{Doing the right science or doing science right?}

"When you rely on incentives, you undermine virtues. Then when you discover that you actually need people who want to do the right thing, those people don't exist because you've crushed anyone's desire to do the right thing with all these incentives" (Barry Schwartz in Zetter 2009 <https://www.wired.com/2009/02/ ted-barry-schwa/> Accessed 12/16/19).

Much of the "How" science is played is discussed within the philosophy of science, and the "What" of science fills books in the sociology of science. The infinite game of science explores the "Why" and the also the "just causes" (Sinek 2019) of science. The "Why" brings us a narrative of science up until this moment, which illuminates its horizon. Science's just causes point us at the thousands of mysteries, the unknowns that scientists confront today; each mystery offers a bit of new knowledge to be discovered, and the benefits of new understanding. Every unknown also carries a moral load, and the need for judgement in pursuit of justice (more about this below), given that there are many consequences to new knowledge.

\section{Mindsets and practices}

The book, Finite and Infinite Games goes into great detail to expose the two mindsets: finite and infinite. These are fundamentally different, and in ways that illuminate many of the issues plaguing the academy today. Here we note only a few key points. First, finite players (players with a finite mindset) play their roles in full seriousness, acquiring their parts as actual and necessary: even when they are always fully free to step out of their parts. They need to forget this freedom in order to play to win. This is really important to keep in mind: finite game players assume their roles as essential parts of who they are, even though they always have the freedom to abandon their role. This mindset lards the role of "scientist" with unnecessary seriousness.

On the other hand, infinite players play with the rules, instead of assuming roles. These rules are constantly changing as players move the boundaries of the game. Infinite play is rule-creating. The players do not need to accept a set of rules to play. Without stable rules, roles make no sense. Best practices do not apply. Every new experiment opens up its own 
horizon. In terms of complexity theory, infinite play demands that you probe, sense and respond (Laloux 2014, Kurtz and Snowden 2003) each time you play.

When an infinite player (someone with an infinite mindset) plays a finite game within infinite play, they do so fully understanding they are simply acting their role, and that they have the freedom to walk away. Yet they still have the capacity to play any finite game to its limit. They can accept the rules in order to play. However, winning or losing has no meaning for them. This may mean they play with greater freedom and abandon, improving their chance of winning.

To remind scientists that their research is a form of infinite play is to reconnect them to the "one long experiment" (Martin 1998) that is science. Recent organizational management theories (Sinek 2019) have put infinite play and sense-making for complexity (Snowden 2002; Kurtz and Snowden 2003; Ito and Howe 2016) at the center of their recommendations for $21^{\text {st }}$ Century organizational governance. Getting good in the infinite play of science could also build skills that scientists can use to govern their labs, universities, and agencies. Infinite play also hones your ability to communicate through narratives.

"In the science world, introducing narrative early and in a substantial way will produce a whole new breed of scientist, able to communicate far more effectively among themselves, as well as with the public. They will also be less prone to subconsciously reach for false positives or present null results in such a boring way that they help perpetuate publication biases against such results" (Olson 2015).

This handbook will help you create new practices that can recenter your university's values and vision around infinite play as a strategy for long-term success. Open science is a cultural platform that will connect infinite players across the globe. You and your organization can join this, or you can continue to play the same bullshit "excellence" games (Moore, et al. 2017; also Neylon <https://www.slideshare.net/CameronNeylon/excellenceis-bullshit>; Accessed Feb. 7, 2020) you take far too seriously today.

\section{Science is a war with one long battle}

The infinite play of science was there with Aristotle and Plato, Bacon and Galileo. With Neuton and Boyle, Einstein and Feynman. And now here, this very moment, with every scientist in and out of the academy. Science play lies beneath the norms that Merton and others have used to delineate science's core ethos. Infinite play stands behind every experimental hypothesis and laboratory method. Every time a scientist battles with some mystery of nature, the infinite game continues. 
Science works toward horizons and not within boundaries. Scientists see boundaries around them and laugh as they violate these. They go beyond. Any scientist can change the horizon of science and modify the rules of science (for example, by improving a method of observation). That horizon, once stretched by a new idea or method, never returns to its former dimensions. Each change in the horizon of science changes the horizon of every scientist.

"The scientist has a lot of experience with ignorance and doubt and uncertainty, and this experience is of very great importance, I think. When a scientist doesn't know the answer to a problem, he is ignorant. When he has a hunch as to what the result is, he is uncertain. And when he is pretty darn sure of what the result is going to be, he is in some doubt" (Feynman, et al. 2005).

The scientist eats unknowns, and is never full. She sweats doubt. The products of science are not science. These can be destroyed or forgotten and science will continue. Science means challenging the known. Scientists understand how little science knows; that the mysteries they face are mighty. Each scientist picks her own mystery, her own just cause to pursue.

"The earth's history has been only long experiment, poorly constrained in a reductionist's eyes. How impoverished the earth would be if had been otherwise" (Martin 1998).

\section{Science is nature made into poetry}

No single scientist speaks for science. No scientist speaks for nature. The speech given by the award winner at the annual convention is not any more scientific than the poster presented by the graduate student. The questions of a student can negate an entire history of discovery.

Unlike the history of society where politics is theatrical and works to close its history (against culture, which keeps this open), the history of science is always dramatic. It is formed by events that must repeat themselves again and again while remaining open to failure, open to a moving horizon that might, and probably will, change and render them false. After that, they join the past history of science and are merely theatrical. One can repeat a failed experiment only as historical theater. The science present moves on in dramatic fashion.

The goods of science inform the knowledge inventory of the world within which science is played. They push science to remake its horizons. They are not unimportant to science but they are not science in the infinite game. Finite-game science players want to own these products, in order to garland themselves with prizes. Prized science goods require durability for the value of their prizes to endure. Finite-game science players choose to 
defend their own goods by silencing others and gathering supporters. They seek a past that is closed and known, with their own goods at its front end.

"One must keep in mind that senior faculty probably hold their current positions through their success in the game, which may or may not have been achieved by using the most ethical ways" (Chapman C.A., et al. 2019).

Infinite science players-who know their own research best-interrogate their own findings in search of a larger knowledge horizon. They push the playing forward and their egos to the side. They open up to collaborations and seek out conversations with those who disagree with their findings.

Prizes bind science to a known past. This past is carried by science institutions, such as those learned societies that sponsor prizes. These societies also need to endure so that the prizes of finite-game science players retain their value. Prize winners and "fellows" carry the weight of ensuring the society persists, warranting the currency of their prizes. However, the continuity of science is not based on an attachment to its past or even it current goods-on the closing of its history-but rather, on a continual openness to surprise, to experiment (Schulz 2011). Science is based on the nearly universal ephemerality of its findings. Science has always been the child of an open history that will never close.

"[W]hat resounds most deeply in the life of Copernicus is the journey that made knowledge possible and not the knowledge that made the journey successful" (Carse).

Science doesn't just have a culture. Science also is culture (in Carse's sense). Like any culture, science is "itself a poiesis, all of its participants are poietai-inventors, makers, artists, storytellers, mythologists. They are not, however, makers of actualities, but makers of possibilities. The creativity of [science] has no outcome, no conclusion" (Carse 1987; paraphrase).

Scientists are ImagiNatives. Poets of the natural world. Makers of possibilities. "It's been said that science fiction and fantasy are two different things: science fiction, the improbable made possible; fantasy, the impossible made probable” (Rod Serling, “The Fugitive”. The Twilight Zone. Season 3. Episode 25. March 9, 1962. CBS.) Science is nature made into poetry.

"The physicist who sees speaks physics with us, inviting us to see that the things we thought were there are not things at all. By learning new limitations from such a person, we learn not only what to look for with them but also how to see the way we use limitations. A physics so taught becomes poiesis" (Carse). 
You cannot do science alone in isolation; do science only in your own mind. This does not mean that you cannot be solitary in your imagination, but only that science happens when you share this with at least one other person. A poet who does not speak has no poetry to speak of. Science happens between and among infinite players.

The infinite play of science allows no personal power or authority. In a finite game, power always requires opposition and an audience. Neither is available within science. In finite games, winning silences the loser. The personal power that a title conveys; this authority means nothing to science, and usually far too much for scientists. Competition feeds arbitrary power in the academy and defeats science itself, silencing the many to praise the few.

Finite games of prestige in the academy are failings of the academy. Finite games of personal influence and authority contradict the inherent authority of science methods. Scientists are known by their names, not their titles. If your method is transparent and well-founded, your science goods need no amplification beyond their public sharing. The audience that power seeks is not found in science. An infinite game allows no audience. There is no vote that can elevate one science good above another.

Science does not belong to any one society. Science flows across the globe. Change for science has no location, it is always everywhere. Change is always surprising, and so never a surprise. "To be prepared against surprise is to be trained. To be prepared for surprise is to be educated" (Carse). To invite and welcome surprise is to do science. Science creates its activities through fluid consensus, not from any established doctrine, but in response to surprises that happen whenever science moves its horizon.

"[As it is in nature, so] also in [science]. Infinite players understand that the vigor of [science] has to do with the variety of its sources, the differences within itself. The unique and the surprising are not suppressed in some persons for the strength of others. The genius in you stimulates the genius in me" (paraphrase) (Carse 1987).

Every science effort begins and ends in surprise. Because the next instant of knowing is always open, the moment of discovery is always surprising. This is a source of joy for the scientist. If the object of research were already known or fully predictable, the research is unnecessary. Reproducibility means that the same effort must result in the same surprise. The first effort exposes the scientist to this surprise. The second time gifts this surprise to science.

In its infinite play, science invests more authority on the rigor of its methods than it does in the sagacity of its practitioners. The results of well-constructed experiments are all 
discoveries, even when the results are null. Each experiment extends the horizon of the game.

\section{Who wants yesterday's findings?}

"If everything we write today already bears within it a future anterior in which it will have been demonstrated to be wrong-headed, we have the potential for a genuine exploration of a new path, one along which we develop not just a form of critical audacity but also a kind of critical humility" (Fitzpatrick 2019).

The final page of science will never be written. A new finding is lightly penciled in after the previous paragraph on the current page. Every infinite player brings their own eraser to this book. Chapters long settled and well considered can be erased in a single day. That is a joy for science. New pages open up then. New horizons emerge. The play accelerates. More players find ways to add new paragraphs to this ledger. Ever since Bacon, yesterday's findings hold less knowledge than tomorrow's.

Scientific surprise is mainly retold as serendipity. Serendipity, the unexpected confluence of curiosity and sagacity, is just another way of announcing that scientists are playing with/in the infinite mode.

"The paradox in our relation to nature is that the more deeply [science] respects the indifference of nature, the more creatively it will call upon its own spontaneity in response. The more clearly we remind ourselves that we can have no unnatural influence on nature, the more our [science] will embody a freedom to embrace surprise and unpredictability" (paraphrase) (Carse 1987).

\section{What is real science again?}

"The notion that academic scientists have to be humble and disinterested... seems to contradict all our impressions of the research world. Scientists are usually passionate advocates of their own ideas, and often extremely vain" (Ziman 2002).

We have all read studies and stories of "actual" science that highlight how scientists live and work in the "real world." Today, scientists labor within the pragmatic circumstances of the increasingly neo-liberal academy-surrounded by an increasingly neo-liberal global economy: a world of intense competition for fame and funding, a space of cumulative advantages for a few, and increasing precarity for the rest. Yet the moments of science, the methodic but often serendipitous event of discovery, yank the scientist back into a different "real" - the real task of uncovering new knowledge about the actual "real world" - the natural universe. To do science is to play with nature. 


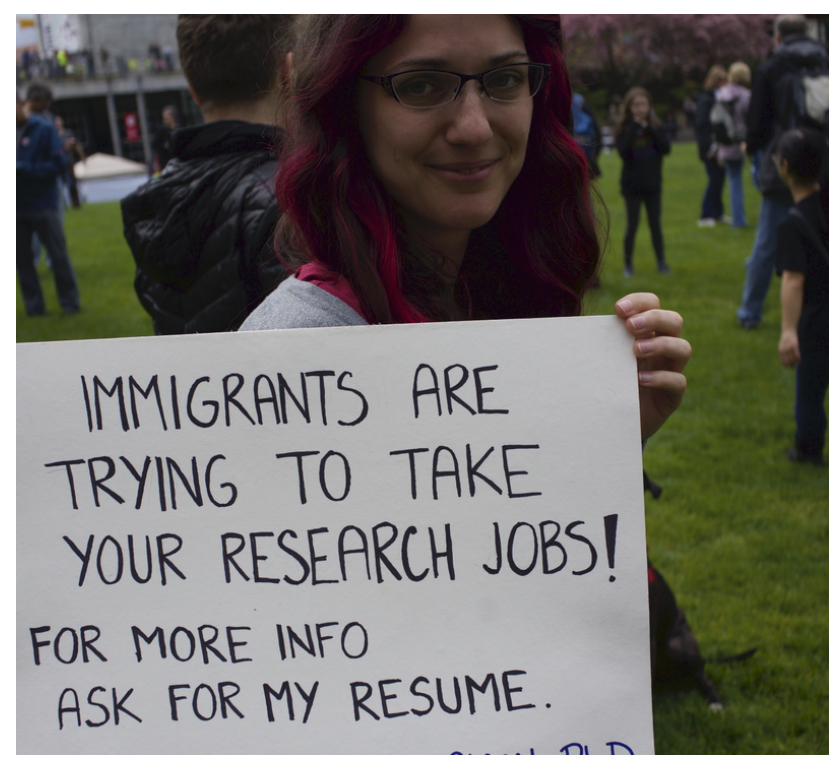

\section{Finite games and infinite science: what's the difference?}

The distinction between playing with an infinite mindset and playing with a finite game mindset allows us to unpack how a scientist might bring an infinite mindset to "actual science:" to all those finite games of power and scarce resources scientists play today in the academy. This handbook is all about changing cultures in the academy. Some of these changes are steps forward into new opportunities, others are steps back into the core of how science was done before the recent neoconservative managers arrived on campus. Let's look at two personas: one, a die-hard finite game player, and the other, a scientist deep into infinite play.

\section{The finite-game player takes on the role of "The Scientist"}

Below are some signs you might be playing science with a finite-game mindset.

- The role of "The Scientist" is just a role. You are free to throw this aside at any time. You always have this freedom. At some point you forgot this fact.

- By forgetting you create a necessity to this role in your life: not for science, however, but for being The Scientist.

- $\quad$ Entering grad school on a fellowship, you identified avenues of influence you could tap into: you picked a famous scholar to be your committee chair and selected a hot research topic, instead of one from your own interests. 
- You had three papers published by the time you completed your PhD. At least one of these used text "borrowed" from a colleague.

- Your old committee chair had an inside track to a funding agency that you learned about and cultivated as a post-doc.

- You jumped into an entry tenure-track position at a different university when your first research proposal was funded, taking your funding with you.

- At annual meetings of your learned society, you work the publisher booths to find a sympathetic editor at a high-prestige journal.

- You push your funded research team of grad students, post-docs, and research staff to make discoveries, or hack the data, to fit the needs of the field's top impact-factor journal.

- You ignore those students who don't perform to your demands, and especially selffunded graduate students, who should realize they don't belong.

- You shift your lab's research focus in response to the funder's new five year strategic plan.

- When you take on peer review assignments, you are particularly harsh on any work that intersects your own but doesn't cite you, while you soaked up any useful information about their research methods and findings.

- You leverage your funded research to minimize your teaching load, and you weasel the chair into handing over your undergrad survey class to adjuncts.

- You use the same textbook for your upper division class that you had as an undergrad.

- You grade easy to avoid hassles with undergrads.

- Your graduate methods seminar class promotes your own methods, and critiques others.

- The platform of your early career wins became a launching pad to grab career advantage over your peers. You search for other early-career winners, and avoid those who aren't.

- You constantly eye openings for jobs at higher ranked universities. You make sure you schmooze their department heavy-weights at learned society meetings. 
- You worm your way into volunteer leadership jobs at your learned society, hoping to fast-track recognition.

- You sit on a couple of major campus-wide strategy committees, instead of curricular or other social committees.

- Your chancellor gives you opportunities to speak at campus events, where you highlight the research findings you've maneuvered to be most glamorous.

- You mold a social media persona around popular science issues.

- You craft a high H-index by having your grad students write review articles, which you attach your name to as first author.

- You haven't done fully original research or used a new methodology in five years.

- You carefully hoard your lab's data, and only publish in journals that do not require open data.

- You evaluate your colleagues as winners or losers, and steer clear of the latter.

- You talk about meritocracy in the academy, and believe that's why you got tenure.

- You laugh off talk of "work-life balance." Your work is your life.

- You fit fully into the role of "The Scientist." As it colonizes your future, the role of "The Scientist" becomes everything you are and ever wanted. But then you realize you haven't yet been elected a fellow of your learned society. You worry that you haven't spent enough time cultivating connections society board members.

- You've never reflected on how your need to harvest your cumulative advantage impacted the quality of your science outputs, nor the career costs of the grad-students you've abandoned because they didn't follow your lead. You never stopped to count the dreams you killed along the way.

- Because you feel you must play The Scientist continually, you are unable to play infinite science. There is no joy in your work. There is a constant fear that your research results will be proven illegitimate.

A few of the the above activities might be pursued as a finite game by a scientist with an infinite mindset (See: Learning infinite science play). Every scientist is confronted by an academy infested by conflicts of interest and internally validated perverse incentives. As open science works to change the culture, scientists must still forge their careers while knowing that what they should be doing is not getting done: "Caught up in being neoliberal 
subjects who operate within the terms of dominant discourses does not suit academics very well. It runs counter to intellectual work. It places us in the impossible situation of existing in a context where what we know we should do is scoffed at as a romantic dream, a fantasy, an indulgence of the past-a love like Othello's of Desdemona" (Davies 2005).

For every finite-game science player who "wins," dozens more need to "lose." Scarcity in the system demands this. "Losers" have their careers side-tracked at some point. Their dissertations do not result in high-impact journal articles. Their post-doc opportunities (if available) become dead-ends. The funding agency denies their last-chance research proposal. They migrate away from research institutions to other jobs in and out of academia. The enthusiasm and hope they brought with them as students no longer sustains the energy they need to compete in the finite games of science. They go off and do other work. This is one reason why science loses every time finite-game science player wins.

\section{B) The infinite player does science and plays with the role of "The Scientist."}

"It took a flight across the equator, a perilous crossing of the Andes and three days down the river in a dugout canoe to bring me to the heart of the rain forest....There is no word but awe for the biological excess of that place, the profusion of life, vivid and complex beyond our grasp. At every turn of a leaf, there are mysteries. There are life forms here that occur nowhere else on the planet and intricate relationships evolved over eons. You might take care not to step on them" (Kimmerer 2003).

Freedom of thought is a fundamental academic freedom. Because science is always shared, this first freedom includes freedom of speech. Freedom is central to infinite play, where boundaries and horizons, rules and roles, histories and futures are all in flux. Freedom of thought is the infinite-minded scientist's chief weapon against the silence of nature. Like water, science flows against nature and finds the low spots where new knowledge lurks. Freedom interrupts scientific rigor and intention with the serendipitous discovery.

The infinite player is fully aware that a finite academy game she agrees to play carries a role she admits only to others. She is never "The Scientist" even when she plays one. She does science. She wears the white coat. She shares her findings, her data, her methods, her ideas. She teaches classes that open up infinite play to her students. She talks about awe and about doubt, about method and precision, and how doing science is something more than doing anything else; and it is more, because she plays with/in the infinite. And when her corner of nature's mysteries remains silent over months and years, she persists. She knows the playing will last when she is gone.

There are finite games in which she has zero interest (to the annoyance of her Chair). She sees no point in crafting a "sexy" P-hacked paper for a high-impact journal. At conferences, 
she spends most of her time on conversation with students at their posters, or with a few colleagues who occupy the same corner of nature as does she. Chancellors and deans fail to recruit her to campus committees. She risks tenure by focusing on her teaching and her idea of research, on her students and their needs, and on the infinite play that fills her mind day and night. If she must leave this university, she will seek out a college somewhere, with the help of her ex-students, and continue to play.

Still, the innovative thrust of her experiments, the transparent rigor of her methods, the quality of her data (which she freely shares) and the unexpected results these reveal keep getting noticed. Despite her inattention to them, her metrics are stellar. In her tenure path, she is a "maverick" and a "connector:" her career is intentionally boundaryless (Dowd and Kaplan 2005). Her generosity is widespread, and well known. She simply lets go of the science goods that are most important to her, knowing that others will remember, and send her new ideas to try out. She is a giver, a genius-maker:

"In Multipliers, former Oracle executive Liz Wiseman distinguishes between geniuses and genius makers. Geniuses tend to be takers: to promote their own interests, they 'drain intelligence, energy, and capability' from others. Genius makers tend to be givers: they use their intelligence to amplify the smarts and capabilities' of other people, Wiseman writes, such that 'lightbulbs go off over people's heads, ideas flow, and problems get solved'" (Grant 2013).

She was denied tenure at her university for ignoring many of the hoops through which she was expected to jump; and immediately hired with tenure at a different university, on the weight and the promise of her research, and the stories about her teaching and mentoring, volunteered from her ex-students. She commonly refuses awards and honors; she calls them distractions.

Even the awe and joy of infinite play can be easily forgotten; scientists can get lost when they play only finite games with scientific methods and organizational power. These finite games pull their logics from other finite games outside of the academy. These logics tear the academy away from the freedoms that science needs to pursue infinite play. The more that the academy is trapped into finite games, the less it gains through open sharing and new opportunities for collaboration and innovation.

\section{The Just Cause(s) of Open Science}

"In life, unlike chess, the game continues after checkmate" (Isaac Asimov).

Open science exists to return the everyday life of scientists to infinite play, to find paths to justice, and to support teaching and research opportunities for scientists everywhere on the planet, in any open institution that will house their work. Open science builds academy commons (plural) where scientists can govern themselves and their resources, maintain 
and care for their goods and each other, provision their work, and build an abundant future for infinite science across the globe.

Infinite play requires and rewards, demands and builds, encourages and exercises practical wisdom inside science. This type of caring, pragmatic wisdom can carry a scientist, a science team, a laboratory, a school, a university, an agency: any all academy organizations, toward open science, where sharing and caring are not reserved for losers. Where there are no winners, only players. And that is the whole point. 


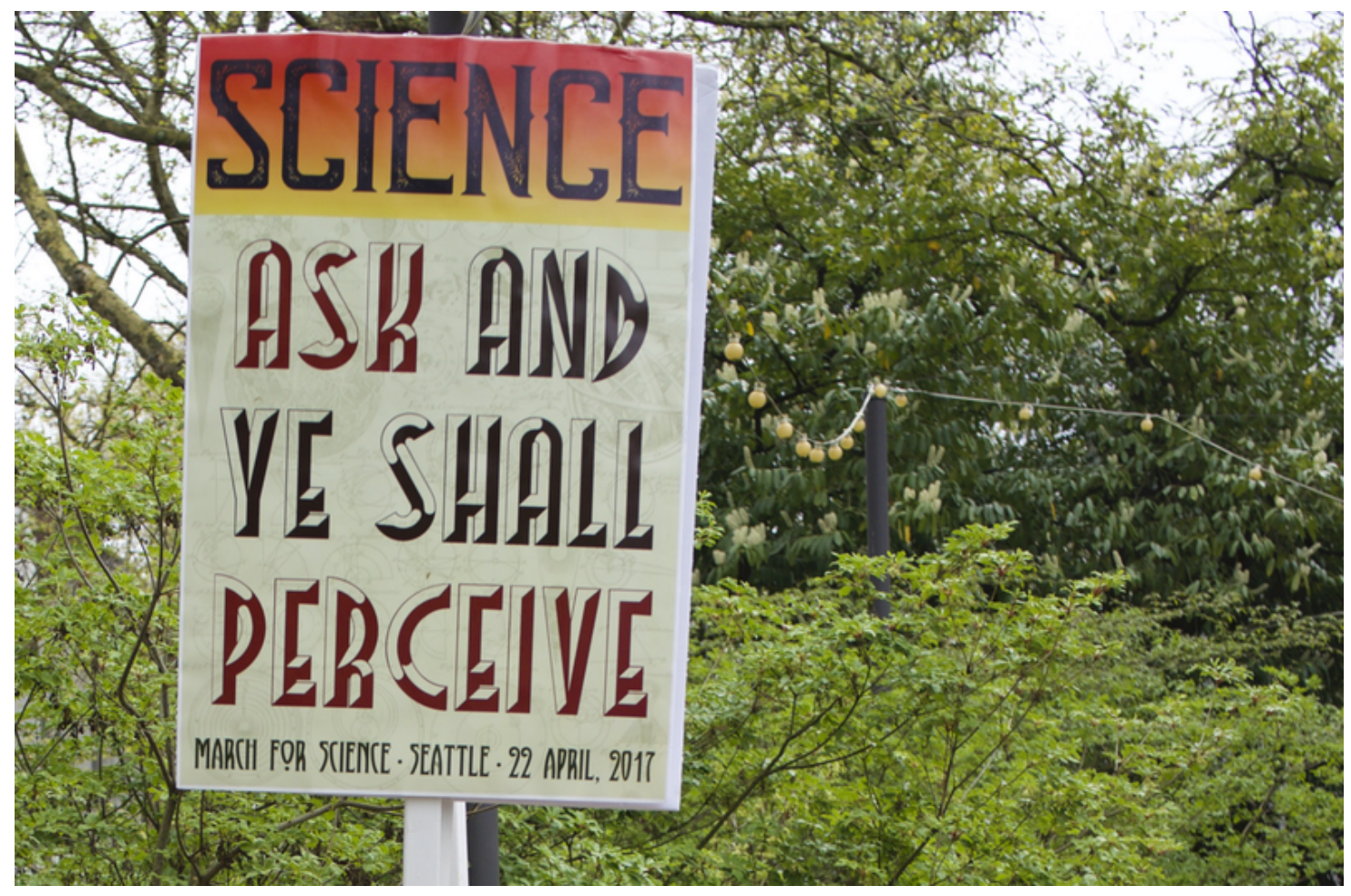

\section{Learning the infinite play of science}

“...The tools we make to build our lives: our clothes, our food, our path home... all these things we base on observation, on experiment, on measurement, on truth. And science, you remember, is the study of the nature and behaviour of the universe, based on observation, experiment, and measurement... The Mushroom Hunters. Neil Gaiman

See: Brainpickings <https://www.brainpickings.org/2017/04/26/the-mushroomhunters-neil-gaiman/> Accessed December 24, 2019.

Albert Einstein describes his infinite play:

"The words or the language, as they are written or spoken, do not seem to play any role in my mechanism of thought. The psychical entities which seem to serve as elements in thought are certain signs and more or less clear images which can be 'voluntarily' reproduced and combined.

"There is, of course, a certain connection between those elements and relevant 
logical concepts. It is also clear that the desire to arrive finally at logically connected concepts is the emotional basis of this rather vague play with the above-mentioned elements. But taken from a psychological viewpoint, this combinatory play seems to be the essential feature in productive thought..." (Einstein 1960; emphasis added).

\section{You Get to Play with Infinity}

After years of study and learning how to learn, you are active in pursuit of the unknowns of the universe. You have acquired all the accumulated information, all the theories, facts, and guesses about your particular object of study. You have mastered the methods, the instruments and the code, you need to query this object, which is now the last teacher you will ever fully need. You have entered the zone of infinite play. You are a scientist, just like Albert.

How do you learn infinite science play? "Infinite science play" may seem like a metaphor for something more "serious": "tackling a complex problem," or "stretching the envelope of our knowledge." This is not so. The use of "play" here is accurate, in the sense that play often: 1) builds and rewards skilling; 2) uses rules and shared limits (time and space); 3 ) and, is open-ended: its outcome cannot be predicted. "Infinite" play points to the universe around us, and our place in this and notes that this particular type of play is fundamentally different from any finite game play.

In infinite play, rules and horizons can and will change. Boundaries are broken. Roles are just labels. Infinite play prohibits winning and losing. Players come and go. Every player will go at some point, but the play moves on. Evolution is one way nature learns its own infinite play. Species come and go. The ecosystem moves on.

Once these particulars are known, then the strategy for playing shifts away from tactics based on winning, toward cooperation, and to efforts to make the play more interesting, to play longer and include more players, to go deeper, to dive into playing. The unknowns you seek to understand are linked in the same way that natural philosophers and scientists have been playing for centuries. Now it's your turn.

As you cannot win infinite play, a good tactic is to discover more intrinsic rewards for playing. Fortunately, the better you get at playing, the more fun you can have. This is something of a secret that your thesis advisor may not have told you: the more fun you have, the more you will play, and the better you will get, and the more fun you can have. Playing better, when it comes to your research, means more innovation, better insights, and improved results. Just ask Albert (ibid) — or Arthur, Paula, Thomas, Steven, or Johannes $<$ https://www.brainpickings.org/2013/08/14/how-einstein-thought-combinatorialcreativity/>. 
Kevin Kelly < the "infinite game" enables technology innovation way back in 2005:

"Our humanity is actually defined by technology. All the things that we think that we really like about humanity is being driven by technology. This is the infinite game. That's what we're talking about. You see, technology is a way to evolve the evolution. It's a way to explore possibilities and opportunities and create more. And it's actually a way of playing the game, of playing all the games. That's what technology wants. And so when I think about what technology wants, I think that it has to do with the fact that every person here-and I really believe thisevery person here has an assignment. And your assignment is to spend your life discovering what your assignment is. That recursive nature is the infinite game. And if you play that well, you'll have other people involved, so even that game extends and continues even when you're gone. That is the infinite game. And what technology is is the medium in which we play that infinite game. And so I think that we should embrace technology because it is an essential part of our journey in finding out who we are" (Kelly $2005<$ https://www.ted.com/talks/ kevin kelly on how technology evolves $>$ Accessed April 12, 2019).

Substitute "science" for "technology" in the above and you will understand why you play.

\section{Look inside for your incentives}

"In academia, a special motivation called 'taste for science' exists..., which is characterized by a relatively low importance of monetary incentives and a high importance of peer recognition and autonomy. People are attracted to research for which, at the margin, the autonomy to satisfy their curiosity and to gain peer recognition is more important than money. They value the possibility of following their own scientific goals more than financial rewards .... The preference for the autonomy to choose one's own goals is important for innovative research in two ways. Firstly, it leads to a useful self-selection effect of creative researchers. Secondly, autonomy is the most important precondition for intrinsic motivation, which in turn is required for creative research..." (Osterloh and Frey 2011).

One of the motivations that "money cannot buy" is the experience of scientific discovery. Whether this is an "aha" moment in the shower or on the bus, a visual experience from an observation, or the result of a computation on data, you get to be the person/team thatright now, this moment-knows something the rest of the world does not. And sure, this new bit of knowing will need confirmation and validation, but in this moment, your passion is rewarded and you find yourself in what social psychologists call an "optimal experience." 
This is not an accident. You have worked really hard to get here. This is why you are driven to be a scientist; "As we have seen, many of the most active participants in these creation spaces are driven by intrinsic motivations-the passion they have for the domain, the satisfaction they feel when solving difficult problems and helping others, or a desire to build their skills and experience base" (Hagel, et al. 2012).

This is an experience that can only come from being skilled, from knowing what you have learned over the years, and from risking failure commensurate to your skilling. Another word for this experience is "flow;"'“Flow is found in using a full measure of commitment, innovation, and individual investment to perform real and meaningful tasks that are selfchosen, limited in scope, and rewarding in their own right" (Mitchell, in Csikszentmihalyi 1992).

How much flow you can experience depends on your own demeanor, on the circumstances of your research employment, and how your organization is governed. Your intrinsic motivations easily can get crowded out when money enters the equation:

"Crowding-out of intrinsic motivation by stick and carrot: Carrots and sticks replace the taste for science (Merton 1973) which is indispensable for scientific progress. A scientist who does not truly love his work will never be a great scientist. Yet exactly those scientists who are intrinsically motivated are the ones whose motivation is usually crowded out the most.... [A] lot of potentially highly valuable research is crowded out along with intrinsic motivation..." (Binswanger 2014).

It's not simply flow that gets crowded out. Money comes with a load of conflicted interests that warp how you configure your science practice. The crowding-out impacts of adding money to (previously straight-forward) moral-choice situations have been experimentally verified (See: Bowles and Polanía-Reyes 2012; Osterloh and Frey 2015; Benkler 2016).

This very common combination of zero-fun - what they call "low flow" - and delayed moral choices- "I know this is wrong, but it makes economic sense to me right now"-describes the state of science when infinite play is interrupted by the logic of the neoliberal marketplace. It probably describes your own lab or department today (Binswanger, M. 2014).

Why should your research be held hostage by perverse incentives that hijack all the fun too? You've worked too hard and know too much to miss the intrinsic joy of infinite play. You need to get the taste for science back into your head, and in the minds of your team. This is why open-science culture change is important.

"The legacy of traditional ecological knowledge, the intellectual twin to science, has been handed down in the oral tradition for countless generations. It passes 
from grandmother to granddaughter gathering together in the meadow, from uncle to nephew fishing on the riverbank, and next year to the students in Big Bear's school. But, where did it first come from? How did they know which plant to use in childbirth, which plant to conceal the scent of a hunter? Like scientific information, traditional knowledge arises from careful systematic observation of nature, from the results of innumerable lived experiments. Traditional knowledge is rooted in intimacy with a local landscape where the land itself is the teacher" (Kimmerer 2003).

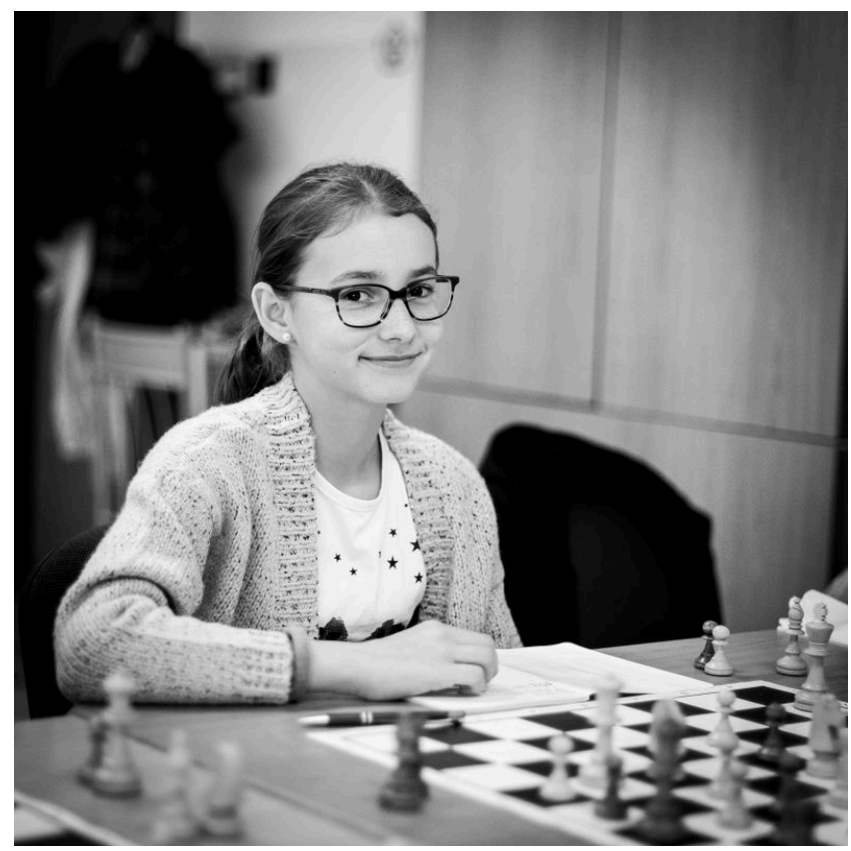

You must play to get better at playing

\section{Playing to learn the infinite play of science}

You must play to get better at playing. The practice of science builds the praxis of science. ["A praxis is a practice that contains the purpose in itself, and is, therefore, the good to strive for"(Klamer 2017)]. Through play you will develop strategies, tactics, processes, and practices, just as you would in a finite game. However, infinite play has its own flavor for these practices: they are durable, non-destructive, and encourage wider play. Seth Godin (2019 <https://www.akimbo.me/blog/s-3-e-14-waiting-for-godiva> Accessed April 16, 2019) provides four key rules (paraphrased here), that apply well to infinite play:

1. Repeatability: what you propose to do needs to be repeatable, not a one-off. Ask yourself: can I keep on doing this? Remember that infinite play has no ending. You research methods must be repeatable to be verifiable, and also falsifiable. You are also repeating what others 
have done. They have passed on their knowledge. Your turn is now. Tag, you are it. Others will come after you. You need to let go of what is most important to you. Your job is to contribute. You invest in open science and others will build on your work.

2. Non-harmful to others: what you propose to accomplish cannot harm others or the planet in the process. This feature is connected to repeatability, of course, but also to a general moral code. "Do no harm." It means non-harm to the careers of other scientists, and neutral or better, positive, impacts on the environment humans need to thrive.

Infinite play is not a zero-sum event. Your success should not be at someone else's expense. The academy needs to refactor over-competitive practices (in funding and promotion) into collaborative ventures. Open science play is not extractive. The opportunities for discovery are abundant.

3. Additive: This is connected to complexity theory and the need for practices to experiment, iterate, and learn. New knowledge is produced in the process. You are impacting the evolution of the infinite-of nature and of the field of knowledge-as you play with this. New complexities emerge. While you are "repeating," each repeat has new results. You experiment and iterate. That's how science is done.

Infinite science play is generative. Its goods are anti-rivalrous. Getting "scooped" is not your problem. Obscurity is your problem. Your process or practice needs to offer a learning curve. You get better at it. You train others in it. They go off and improve the process. Then they can teach you new things.

4. Non-secretive: If you need to keep your process or practice a secret for it to work, then it will fail. Infinite play means inviting others to join. Secrets are for finite games. Infinite play runs on sharing. Open science is democratic at its core. Fierce equality means sharing with everyone. Open science is generous.

This is not all of what you need to play with/in the infinite. Just a taste. Ahead, you will see how open-science based infinite play restores science's normative drivers, marginalizes perverse incentives, embraces emergent complexity, nourishes practical wisdom in the academy, and fosters innovative serendipity.

"The men go running on after beasts.

The scientists walk more slowly, over to the brow of the hill and down to the water's edge and past the place where the red clay runs.

They are carrying their babies in the slings they made, freeing their hands to pick the mushrooms." The Mushroom Hunters. Neil Gaiman See: Brainpickings <https://www.brainpickings.org/2017/04/26/the-mushroomhunters-neil-gaiman/> Accessed December 24, 2019. 


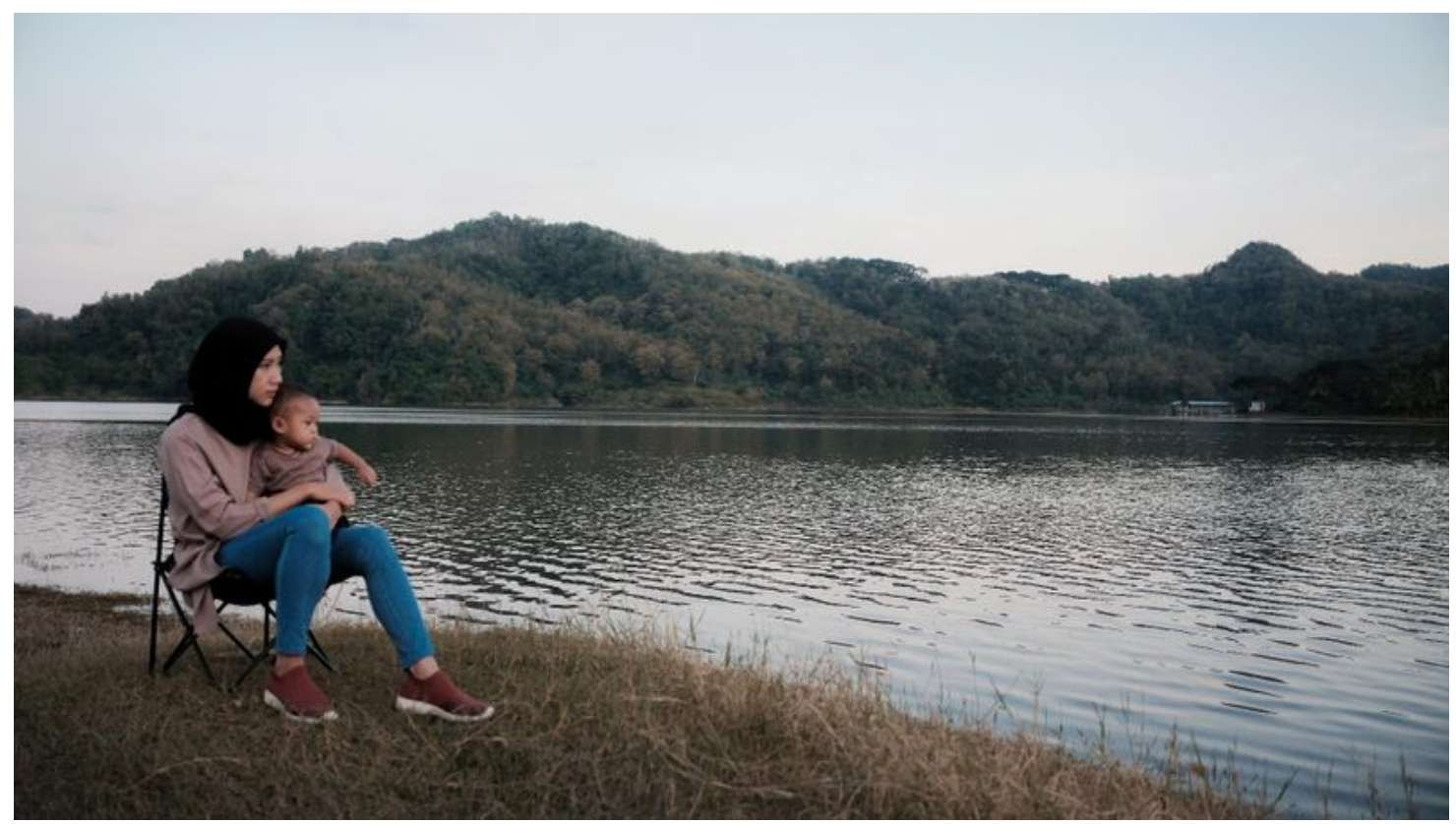




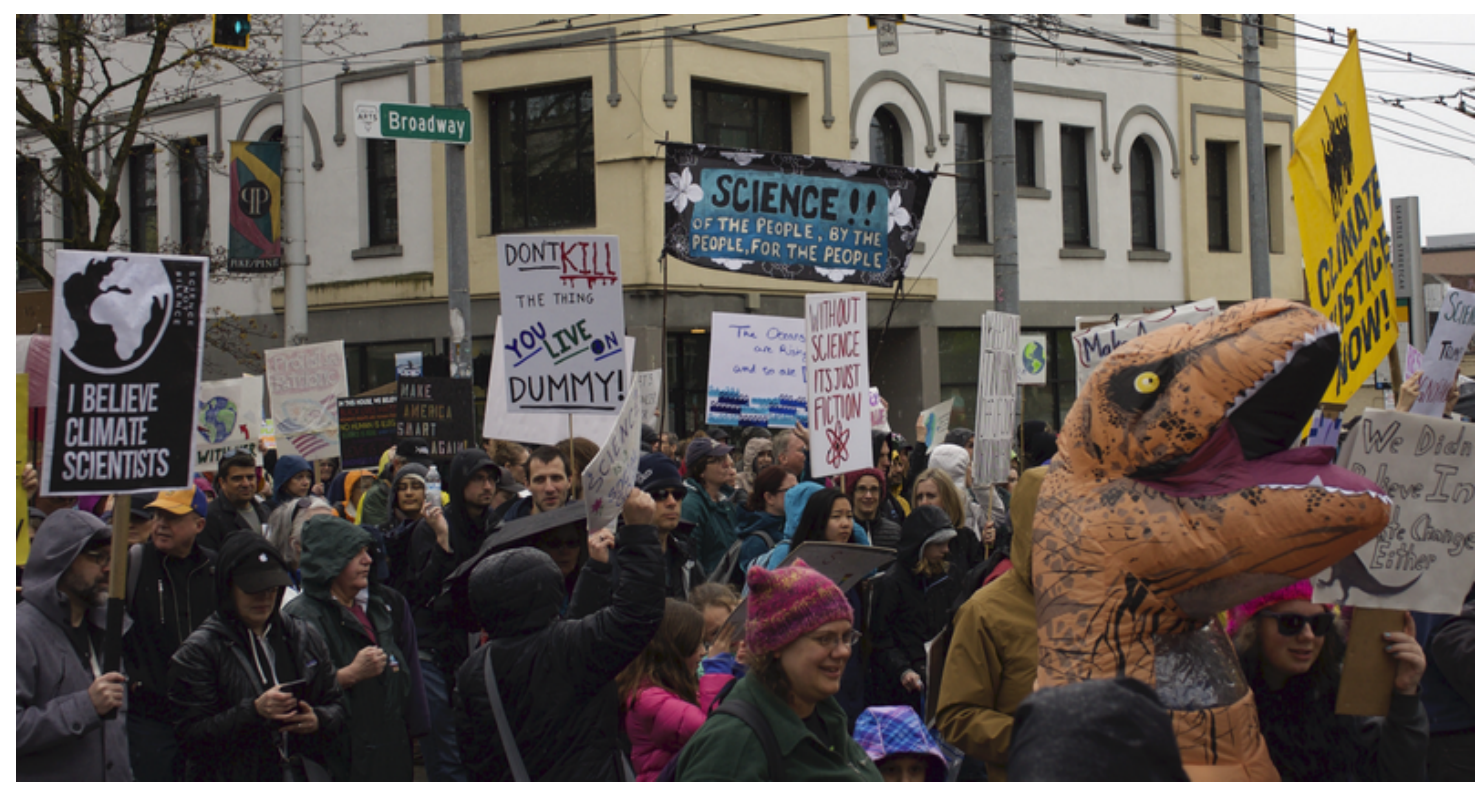

\section{The practical wisdom of doing science}

"If you compromise your integrity and principles on minor issues, it gets easier to make bad choices on the big issues" (Dunwoody and Collins 2015).

"Do the right thing. It will gratify some people and astonish the rest" (Mark Twain, attrib.).

The current research on "wisdom" defines this in approximately as many ways as there are wisdom researchers (Sternberg 1990). Fortunately, we do not need to lock down a definition of wisdom here to see its lack in the academy, and the benefits of promoting this as one more measure of what it means to do science and to be a scientist today. Most of the research on practical wisdom confirms what you already think about this. People who are wise in this way know how and when to do the right thing in a broad range of circumstances.

It's not all that simple, of course. Doing the right thing is not merely "being right," and most certainly is not thinking you're right, and convincing everyone else how wrong they are. Practical wisdom operates through the infinite play of open, complex intellectual and normative choices, and conflicting and ambiguous circumstances.

The "right thing" is rarely an easy binary operation and it often requires insights and emotional commitments beyond a simple logic. It may also be an action that goes against the immediate best interests of others or the wise person. "[M]any who have written on wisdom have identified it with the ability to develop and defend good judgments about the 
difficult, wicked-decision problems characteristic of adult life" (Kitchener and Brenner 1990).

Mostly, practical wisdom examples involve interpersonal or socioeconomic decisions. These examples map how individuals wend their life together with others and the world. This part of practical wisdom encompasses careers in and out of the academy. You can explore the more general forms of practical wisdom literature elsewhere (Practical wisdom: The right way to do the right thing by Schwartz and Sharpe (2010) is a good place to start, also Barry Schwartz's TED talk [Accessed January 7, 2020] or his WIRED interview). So, what forms of practical wisdom are peculiar to doing science?

In finite games, where arbitrary scarcity and external incentives warp the moral fabric of interaction, practical wisdom often gives way to self-promotion strategies. Somebody has to lose. Actually, most people need to lose in order for winning to matter. In the infinite play of science, nobody wins or loses, and the main strategies include sharing knowledge and adding new players to the mix. Science loads additional wicked-problem solving on top of the adult life problems of work and home. Wise researchers confront the silent unknowns of a complex and emergent natural world.

The ability to start from observations and data about the world and transform these into information, knowledge, and understanding is an inherently moral activity; each bit of new knowledge-big or small—changes existing rules for every scientist and expands the envelope of possible human action. Medical science practice is often cited as a key discipline in need of practical wisdom because of its everyday moral decision making (See: Branch and Mitchell 2011; Kaldjian 2010; and Jeste, et al. 2019). All science domains are similarly implicated when they enter into infinite play.

\section{How does practical wisdom improve research?}

Judith Glück (2017) makes a case for practical wisdom in the academy. The first behavior is a desire for a deep understanding of complex emergent systems, instead of a personal claim about some potentially universal truth. This is precisely the difference between the infinite play science, and playing a finite zero-sum game. "Over time, wise researchers' desire to thoroughly understand should lead them to develop an extraordinary amount of knowledge: a broad and deep integrative understanding of a subject matter that includes a keen awareness of what they do not (and may never) know" (ibid). In short, they become intellectually humble.

“[Humble intelligence is] a method of thinking. It's about entertaining the possibility that you may be wrong and being open to learning from the experience of others. Intellectual humility is about being actively curious about your blind spots. One illustration is in the ideal of the scientific method, where a scientist 
actively works against her own hypothesis, attempting to rule out any other alternative explanations for a phenomenon before settling on a conclusion. It's about asking: What am I missing here?" (Resnick 2019; Accessed June 7, 2019).

Practical wisdom in the academy is built upon the humble intelligence of each scientist. As science becomes more collaborative, networking and teamwork bring new demands on the practical wisdom of every member. To review, Tangney (2000) proses that intellectual humility requires five abilities:

A. the ability to acknowledge mistakes and shortcomings;

B. openness to perspective and change;

C. an accurate view of the self's strengths;

D. ability to acknowledge and experience life outside the direct consciousness of the self; and,

E. the ability to appreciate the worth of all things.

Each of these abilities adds value to any academy collaboration effort. Open science captures this value by promoting equality of access and networked collaborative opportunities.

\section{Greater good: Delving into the Just Causes of science}

The second wise-researcher behavior is a high level of concern and care for the "greater good": for the welfare of the entire "Republic of Science" (Polanyi 1962), of the next generation of scientists, and of the planet:

"Wise researchers will be concerned with the well-being of others, ranging from their students to the world at large. Inside the university, wise researchers will care about the quality of their teaching.... Wise researchers are also caring, generative mentors who seriously work on supporting the career development of their mentees and genuinely enjoy their success" (ibid).

The care that wise researchers bring to their science reflects their ability to set their own ego aside. Many of the distinctions between practical wisdom and general intellectual accomplishment pivot on this attention to the greater good: to the underlying reasons, the overarching effects, the larger, messier, more complex consequences of new knowledge.

Open-access science publishing can support the wise researcher looking to share their whole research in exchange for access to the research of their colleagues. New evaluation tools that reward ethical behaviors and a much greater variety of goals and social activities 
would support the efforts of those with a recognized concern for the welfare of their mentees and for the research success of others. Assessment tools that capture a diverse and incremental landscape of work outputs and socially-supportive activities are already being developed in the workplace outside the academy (See: Buckingham and Goodall 2019).

Practical wisdom is also an antidote to assholes in the academy. The Handbook deals with assholes elsewhere (See: The Zero-Asshole Zone). Most wisdom research concludes that you cannot have practical wisdom and also be an asshole or evil (See: Stanford Encyclopedia of Philosophy: Wisdom, 2002). In part, this is because you cannot have practical wisdom without actually using your practical wisdom. As noted elsewhere in the Handbook, much of the assholic behavior in the academy is learned and rewarded today; and so, most academy assholes are not irredeemable. They learn and use bad behavior to win the finite games currently infecting the academy. They can unlearn these behaviors and gain some practical wisdom over time. Some, however, are true assholes. These will resent and resist change when your new open-science cultural practices no longer support their bad behavior.

How do we build the practices of humble intelligence and a care for the greater good into the academy? Such is the project of this Handbook, a guide to changing culture in your precinct of the academy, wherever this is. Open science builds fierce equality into the academy as a normative behavior that expresses intellectual humility and inclusiveness. In the infinite play of science new knowledge can be found by anyone in the Republic of Science. Open science uses demand sharing to support the greater good. As a sharing economy, the academy's goods gain value across time and space.

The starting point for culture change is, as always, changing yourself. Everyone can gain practical wisdom, even tenured faculty. Start today. Be wiser tomorrow.

\section{Wisdom learning starts in childhood, but need not end there.}

Some researchers point out that a fair amount of practical wisdom is learned throughout childhood, mostly during play (See: Feist 2006; Brown 2009; Carlson and White 2013; and Sharma and Dewangan 2017). If you are no longer a child as you read this, do not worry, you can still catch up. To begin with, play is always available even for adults (See: <https:// eachother.org.uk/the-right-to-play-adults/> Accessed January 5, 2020).

While the "skills" of practical wisdom—and wisdom in general-cannot be gained through the same type of specific practice as, say, a violin, or a golf swing, practical wisdom is similarly experience-based (Cantrell and Sharpe 2016). Like most cultural practices, you can get better at practical wisdom through practice, it is not an inherent trait. You are not born with all the wisdom you can have nor what you might really need to use for your career in the academy. 
Glück and Bluck (2103) demonstrate a model for acquiring personal wisdom based on a "strong sense of mastery, high levels of openness, a reflective attitude, and emotion regulation skills combined with empathy." This "MORE" model can be used in "leadership" curriculum development at the undergraduate level (Sharma and Dewangan 2017). The Phronesis Project [Accessed January 8, 2020] at the University of Virginia School of Medicine is pioneering practical wisdom training in its curriculum. Just remember that you can also have fun while becoming wiser. You enjoy learning, after all, so learning practical wisdom is an opportunity to master another life skill:

"Another value of science is the fun called intellectual enjoyment which some people get from reading and learning and thinking about it, and which others get from working in it" (Feynman, et al. 2005).

Schwartz and Sharpe (2010) find that institutions that manage behaviors through rigid rules or laws crowd out opportunities for members to gain wisdom from self-governed interactions. "[I]t's important to resist those rules and incentives that eviscerate discretion and threaten wisdom. That's why we need to reform those institutions that are driving wisdom out." It could be that your institutions would benefit from more democracy and healthy arguments, and fewer rules. The Handbook sections on Learning Organizations are a good place to start. 


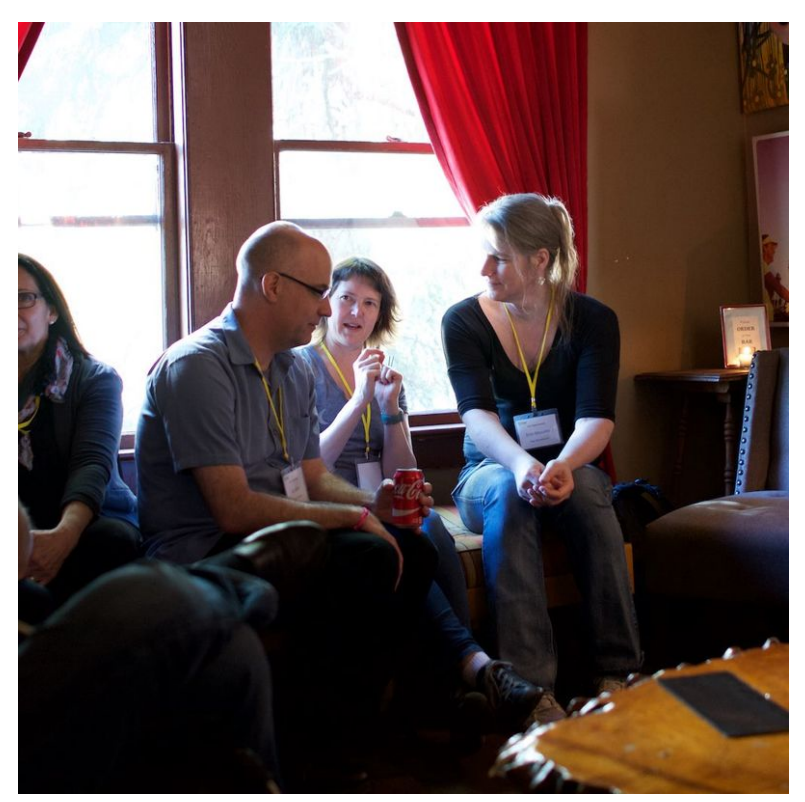

"Innovations in how we conduct conversations should be treated as art" (Schein 2013).

"Focusing on conversation highlights the need for generosity to be continually renewed in order to function. Moreover, it points to the things we owe one another, the things we owe our colleagues, and also the things we owe those publics whom we hope to engage. Conversation imposes an obligation that cannot be easily concluded, that asks me to open myself again and again to what is taking place between us. Conversation thus demands not that we become more giving, but instead that we become more receptive. It requires us to participate, to be part of an exchange that is multidirectional. It disallows any tendency to declare our work concluded, or to disclaim further responsibility toward the other participants in our exchange" (Fitzpatrick 2019).

\section{Knowing and conversation in the academy}

First we turn to look at "knowing," which is a practice intrinsic to scientific innovation and creativity. "Knowing," as it is used here, has its own literature in the business-management world. Like "culture," knowing is always shared. Elsewhere we learned about celebrations of your open-science culture. Here we will look at what Isaac Asimov called "cerebration sessions": events planned to encourage the "folly of creativity," in small, informal groups. These events trigger (when successful) shared knowing. Note: Asimov also noted that the subsequent written outputs for these sessions are incidental. What matters is the content of conversations in the room. 
"Joviality, the use of first names, joking, relaxed kidding are, I think, of the essence - not in themselves, but because they encourage a willingness to be involved in the folly of creativeness....

I would suggest that members at a cerebration session be given sinecure tasks to do-short reports to write, or summaries of their conclusions, or brief answers to suggested problems - and be paid for that, the payment being the fee that would ordinarily be paid for the cerebration session. The cerebration session would then be officially unpaid-for and that, too, would allow considerable relaxation" (Issac Asimov 2014: MIT Technology Review; Accessed March 8, 2020).

\section{You already know knowing, you just don't know it yet}

The recent work of John Seely Brown and others, coming out of organizational knowledge theories in the mid 1990s (See: Boland Jr. and Tankasi 1995), has added (or recovered) a cultural angle on knowledge management which includes not only knowledge, but knowing: because "the interplay between knowledge and knowing can generate new knowledge and new ways of knowing" (Cook and Brown 1999). Instead of organizations stewarding an inventory of knowledge objects, what they need to do is open up contexts and spaces: events for knowing (Thomas and Brown 2011).

"In this way, conversation affords more than an exchange in which the net sum of knowledge remains the same; it dynamically affords a generative dance within which the creation of new knowledge and new ways of using knowledge is possible.

Engaging in such conversation is a practice that does epistemic work; it is a form of knowing. Knowing entails the use of knowledge as a tool in the interaction with the world. This interaction, in turn, is a bridging, a linking, of knowledge and knowing...[Which] makes possible the generative dance, which is the source of innovation. The generative dance, within the doing of work, constitutes the ability to generate new knowledge and new ways of using knowledge-which knowledge alone cannot do. And which the organizations of the future cannot afford to neglect" (Cook and Brown 1999).

These events for knowing are where organizations do their "sense-making" activities, and where scientists collaborate in conversation to solve-to make sense of-the emergent complexities of nature. Scientists use knowing practices every time a new experiment is made. Each time the scientist creates a new test to interrogate a piece of unknown nature, she hopes to distill a bit of new knowledge and a ray of understanding that might lead to new knowing. They share this knowing in conversations with their colleagues. 
While the "official record" for a new discovery might be a published paper, open science works to accelerate sharing by promoting preprints that open up immediate opportunities for scientific conversations across the internet.

\section{Conversations power scientific discovery}

“[N] etworked markets get smart fast. Metcalfe's Law, a famous axiom of the computer industry, states that the value of a network increases as the square of the number of users connected to it—connections multiply value exponentially. This is also true for conversations on networked markets. In fact, as the network gets larger it also gets smarter. The Cluetrain Corollary: the level of knowledge on a network increases as the square of the number of users times the volume of conversation. So, in market conversations, it is far easier to learn the truth about the products being pumped, about the promises being made, and about the people making those promises. Networked markets are not only smart markets, but they're also equipped to get much smarter, much faster, than business-as-usual" (Levine, et al. 2009 [1999]. Emphasis added.)

The very first one of the ninety-five theses of the Cluetrain Manifesto (ibid) says this: Markets are Conversations. The "markets" for research knowledge in open science connect to the emergent abundance of research artifacts in repositories across the globe. But the knowledge that powers discovery right now lives only in the conversations available across networks of scholars. Buckheit and Donoho (1995) make the point that scientific articles rarely hold the scholarship they claim to convey: rather they are "merely advertising of the scholarship."

The solution is two-fold: better ways of publishing results that reproduce more of the method, data, software, and ideas (open science looks to go "beyond the PDF"); and more conversations quicker and across a wider range of internet-enabled media, including online direct conversations among peer-to-peer networks. As we will soon see ( $\underline{\text { Science happens }}$ elsewhere), these networks create virtual "rooms" that are smarter than any of their inhabitants. Following the Cluetrain Corollary, we can assert the following:

"The level and quality of current knowing in any science discipline increases as the square of the number of scientists times the amount of available conversation."

Through Demand Sharing and Fierce Equality, open science resets the norms for research conversations across the planet. Today, virtual science organizations can be easily bootstrapped through platform cooperatives to support active collaborations across institutions and continents.

Extra Credit: For those of you who follow recent French philosophy, these knowing events are the center of the process from which truths emerge in the philosophy of Alain Badiou. 
"[A] truth is sparked by an event and spreads like a flame fanned by the breath of a subjective effort that remains forever incomplete. For truth is not a matter of theory but is a 'practical question' first and foremost: it is something that occurs, a point of excess, an evental exception, 'a process from which something new emerges'..." (Bensaïd 2004; Accessed March 10, 2020).

Of course you do not need to read French philosophy to understand what Asimov and Badiou are telling you: one great conversation (perhaps over beer at a conference, or online on a teleconference) with a colleague about the intersections of your research can be more valuable-can spark more truths about your object of study—-than any article or book in your library.

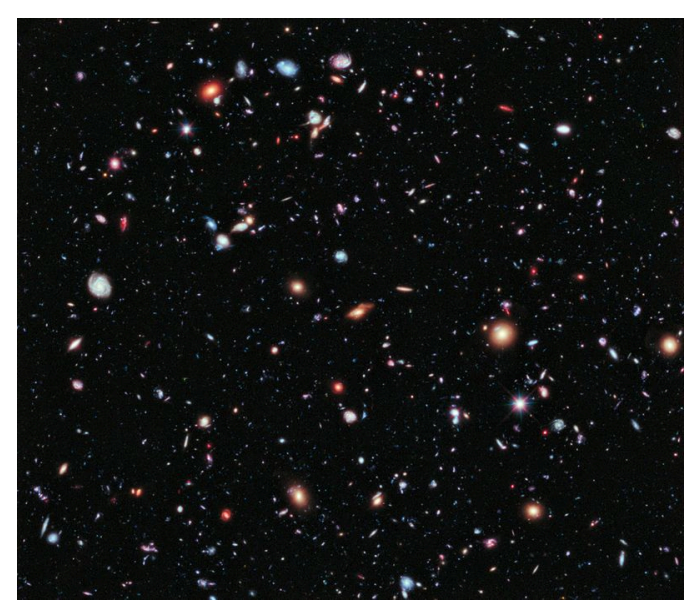

There are are lot of elsewheres out there.

"The smartest person in the room is the room itself: the network that joins the people and ideas in the room, and connects to those outside of it" (Weinberger 2011).

\section{Science happens elsewhere}

Below, you will discover the "double-loops" of organizational learning, and the cultural practices that build and sustain these. But why are these important for science and the academy? Can't we just keep doing things the old way? In fact, you can bet there will be two kinds of academy organizations going forward: those standing still (or worse: See: Is my learned society obsolete?, below), and those diving into the networked opportunities of open science. 
Goldman and Gabriel (2005) penned the phrase: "Innovation happens elsewhere" to capture the value of open-source software communities. In the academy, it doesn't matter if you are at Oxford or in Oxnard, almost everything you need to know to make the next step in your research is also being considered at this moment: somewhere else.

In the academy, this "beyond" is a global intellectual commons now becoming abundant with open data and accessible-and reproducible (Crick et al 2015)-research results. Using online peer production methods (Benkler 2016), the academy can optimize the value of this commons for innovation, knowledge, and growth. "[P]eer production practices [are] highly adept at learning and experimentation, innovation, and adaptation in rapidly changing, persistently uncertain and complex environments" (ibid).

The only competition your academy organization has is within itself. As other institutionsincluding new virtual science organizations-work to continuously improve on their work, your team needs to focus on leveraging the learning engine of double-loop governance to get better than your yesterday. In Learning infinite science play, winning means accelerating your team's learning and sharing capacity through what Hagel and Brown (2011) call a "creation net" for open innovation. Standing still is not an option when the research world is exploding somewhere else. This "explosion of creativity is taking over more and more of our world. Everyone involved in it is at the same time a producer and a consumer, a worker and a manager.... Progress in most academic disciplines now seems to move at the speed of 'instantaneous,' with discoveries building atop one another at a dizzying pace" (Ito and Howe 2016).

\section{Creation networks: open science's network effect}

A creation network is enabled by a certain quality of learning within social interactions, a greater quantity of information flows (and/or a greater attention to these), an availability of interpersonal trust (based on demonstrated skills and commitment), and an environment of reflexive involvement: all benefits of belonging to a community-led doubleloop governance. "[I]nstitutions will need to become much more selective in their efforts to protect existing stocks of knowledge and much more adept in using their stocks of knowledge to contribute more actively in creation nets and to plug into promising flows of knowledge" (Hagel and Brown 2008). Data-intensive science (Hey et al 2009) in a whitewater world of global research demands a nimble governance for its teams, labs, networks, societies, universities, and agencies.

\section{Know enough to know enough}

When your academy organization looks to innovate-or when your personal research is looking to find the right question to ask-in a world where multiple/large data/ information inputs, and international science discoveries are coming on line, how can you 
stay ahead of this emergent complexity? One way to look at this problem is through Ashby's principle/law of requisite variety, coming from cybernetic management. Ashby's law notes that unless the control system has at least the variety of the environment it controls, it will fail; which actually means that some part of the environment will be controlled elsewhere.

You need find ways to join the greater "science elsewhere." Elsewhere is where other science teams are now using their infinite play mindsets in collaboration, asking the questions that their networked teamwork generates. Elsewhere there are flows of information being shared across the planet. That is a great reason for new creation networks in the academy: for open science sharing across the academy.

Elsewhere is where innovation happens; because unless you can corral the inherent variety of the problem you face, it will be too complex for your team to innovate a response. If you are not engaged with the open-science elsewhere that is opening up today, your team will suffer. You can either go out and hire a bigger team (good luck talking your chancellor or the NSF into that), or you can borrow enough requisite variety just long enough to bring your own team up to speed by starting up or hooking into an online creation network. You can join the sharing economy, play infinite science, and get better at it every day. Or you can rest on your (bullshit) reputation and keep on thinking the world will come to you.

When members are given license to form working teams across organizations, they also expand the extent of where their research adjacent possible is found; creative interactions and new knowledge become predictable outcomes. The larger the room, the smarter it gets. Find the room to nurture your research.

\section{When the adjacent possible is a globally available}

The "adjacent possible" is a notion that comes from biological theories of coherent change. It describes how the surrounding environment tucked between stasis and chaos provides a resource of available change. The adjacent possible enables, and almost guarantees, certain changes (while ruling out others) out of potentially infinite play of innovation.

"Biospheres, on average, may enter their adjacent possible as rapidly as they can sustain; so too may econospheres. ... [T] he hoped-for fourth law of thermodynamics for such selfconstructing systems will be that they tend to maximize their dimensionality, the number of types of events that can happen next" (Kauffman 2000). Every new piece of information, each new proto-fact, expands the horizon of infinite science play. The more scientists that add this new fact to their knowledge, the larger their mutual adjacent possible becomes. 


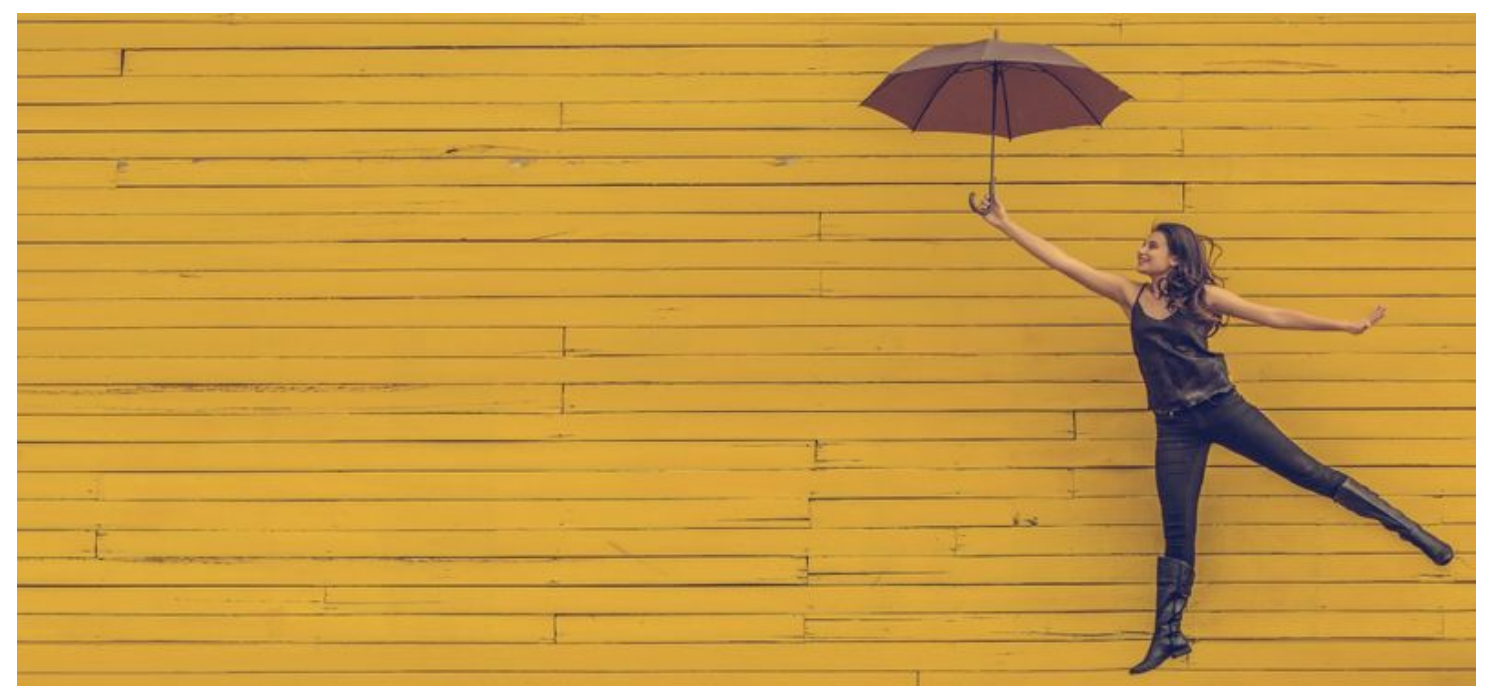

How much joy do you get from your research?

\section{Joy, fun, and love}

\section{Open science is so much more than open access}

"Science functions best when scientists are motivated by the joy of discovery and a desire to improve society rather than by wealth, recognition, and professional standing. In spite of current pressures, it is perhaps remarkable that many scientists continue to engage in selfless activities such as teaching and reviewing, decline to publish work that doesn't meet stringent standards for quality and importance, freely share reagents and knowledge without worrying about who gets the credit, and take genuine pleasure in supporting the efforts of other investigators. Such individuals should be recognized and emulated" (Casadevall and Fang 2012).

"Numbers have many charms, unseen by vulgar eyes, and only discovered to the unwearied and respectful sons of Art. Sweet joy may arise from such contemplations." Charles Babbage circa 1825, quoting French Mathematician Élie de Joncourt, circa 1735 (Gleick 2011).

"Making a better, more sustainable institution, in other words, requires us to move away from quantified metrics for meritorious production - in fact to step off the Fordist production line that forever asks us to do more - and instead to think in a humane fashion about ways that we can do better. Better often in fact requires slowing down, talking with our colleagues and our communities, and most importantly, listening to what others have to say. Better requires engagement, 
connection, sharing, in ways that more nearly always encourages us to rush past. Turning from more to better goes against some of the ingrained ways of working we've adopted, but that turn can help us access the pleasures - indeed, the joys - of our work that life on the production line has required us to push aside" (Fitzpatrick, April 26, 2020; Accessed September 2, 2020).

\section{Start here with joy}

While writing this handbook, it became clear that, as a life-way-as a career that is also an avocation-science today needs to rekindle the internal emotional goods that have long been wellsprings for creativity and innovation across the lifetime of the scientist. Science is the hardest thing humans can do, in terms of the challenges it faces, and the obstacles to resolve these. There is no shortage of hard work, long hours, and disappointments available to the scientist. These have been with science since the time of Francis Bacon. Science is serious. We can take that as given. Science faces many of the hardest and most meaningful questions humans have managed to ask themselves and the universe. What is the origin of life? What is matter made from? Why must we die as we do? But science was never only "serious" in its practice. Scientists get to learn infinite play in their job, a pursuit that opens up to awe and wonder - and joy - at any time. "Joy has a component, if not of morality, then at least of seriousness. It signifies a happiness which is a serious business. And it seems to me the wholly appropriate name for the sudden passionate happiness which the natural world can occasionally trigger in us, which may well be the most serious business of all" (McCarthy 2015). Yes, science is serious, but so too is joy.

"Too much of the OA [open access] discussion is grim, utilitarian, and problemoriented. We should complement it with discussion that is joyful, curious, and opportunity-oriented. Serious problems don't rule out beautiful opportunities, and one of the most beautiful opportunities facing OA is that certain strategic actions will solve serious problems and seize beautiful opportunities at the same time." Peter Suber (2012)

Open science will almost necessarily be more joy-full than the science you've been doing. Some of this comes from its inherent generosity, and the gratitude you feel at the generosity of others when they share openly the science findings that help your research. "Gratitude is a powerful emotion. We declare that we are satisfied. We can drop our search for more; in this moment, we have everything we need. Out of that fullness, other emotions naturally bubble up. We tend to get in touch with joy and generosity, and we treat others with love and care" (Laloux 2014).

Open science enables infinite play. You will be challenged there when nature stays silent to your questions, but you will also find joy. "There can be occasions when we suddenly and involuntarily find ourselves loving the natural world with a startling intensity, in a burst of 
emotion which we may not fully understand, and the only word that seems to me to be appropriate for this feeling is joy" (McCarthy 2015) (See also: Popova; Accessed May 31, 2020).

Certainly, there is some little joy in those finite games that scientists play today. However, this type of joy is kept scarce through the logic of "science as a race." This logic informs scientific research as a series of races, each one ending in the form of some achievement that can be owned by the scientist, and in extension by their home organization. "The contradiction of finite play is that the players desire to bring play to an end for themselves" (Carse 1987). In order to win, and to feel this variety of joy, they need to hold up their discovery-as a distinct, independent object-for public notice. They seek an audience, and arenas-certain journals, learned society prizes, funding agencies, campus administrators -where their personal winning can be acknowledged with some special notice or title. "If finite players acquire titles from winning their games, we must say of infinite players that they have nothing but their names" (ibid). The winner's joy is amplified by the number of losers around them. They have succeeded where so many others failed. But the joy of these distinctions is momentary. The next race has already begun.

"The revelation of suddenly seeing what I was blind to only moments before is a sublime experience for me. I can revisit those moments and still feel the surge of expansion. The boundaries between my world and the world of another being get pushed back with sudden clarity an experience both humbling and joyful"

(Kimmerer 2003).

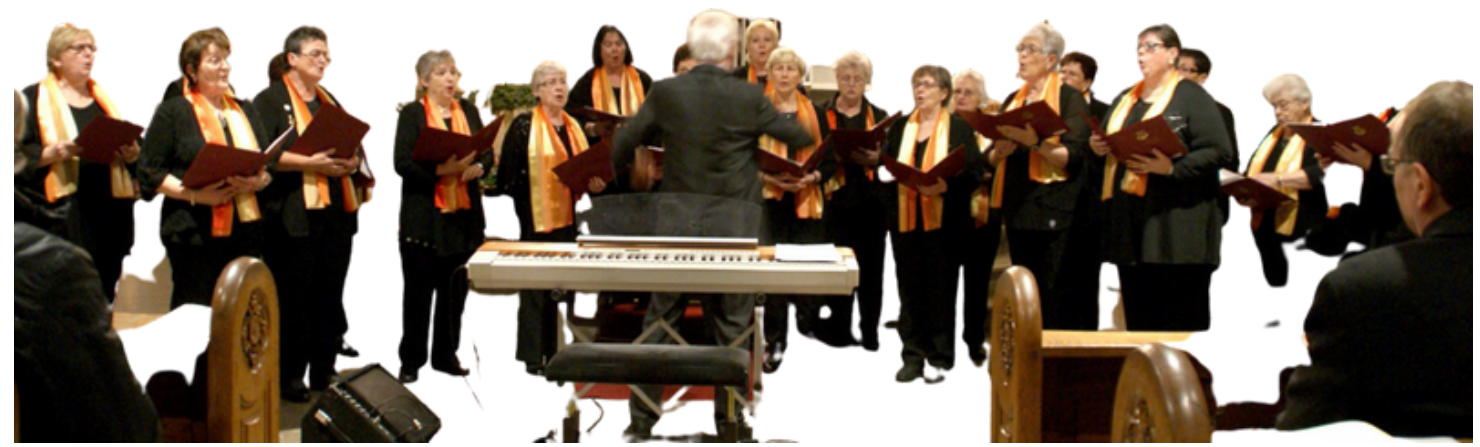

\section{Joy in abundance}

The joy of open science is like the joy of a choir while creating the music they sing together. This joy is fully shared, as a form of collective virtuosity (Accessed June 9, 2020). The better the singers' voices blend into a single voice, the finer their song sounds. Should the choir grow larger, the joy only multiplies. Should the song grow longer, the joy only expands. "The paradox of infinite play is that the players desire to continue the play in others. The paradox 
is precisely that they play only when others go on with the game. Infinite players play best when they become least necessary to the continuation of play. It is for this reason they play as mortals. The joyfulness of infinite play, its laughter, lies in learning to start something we cannot finish" (Carse 1987). Open science is a song with a choir anyone is welcome to join (with some real training behind them), a song that doesn't end when any one voice becomes silent.

"Specifically, joy may be thought of as delight that arises in response to a source of meaning or value in life. Delight describes a pleasant emotion, conveying the positive valence of joy. Connecting this to a matter of meaning or value differentiates joy from other positive emotions, such as happiness, a more general case in response to anything pleasant; amusement, in response to something entertaining; gratitude, in response to receiving something; pride, in response to accomplishing something; interest, in response to engaging in something, etc." (Krumrei-Mancuso 2019).

Under certain circumstances, doing science opens up opportunities-events-for a delight that comes from connecting to nature. "A lot of the time, when you do Math, you're stuck. But you feel privileged to work with it. You have a feeling of transcendence and feel like you've been part of something really meaningful." Akshay Venkatesh (ICM 2018; Accessed June 1,2020$)$. Science organizations can be governed to encourage, enable, and celebrate these events.

More importantly, individual scientists need to develop their capacity for joy, a capacity they might have had as a child only to lose during their schooling. Like practical wisdom (The practical wisdom of doing science), you can get better at finding moments of joy in your research and teaching. Of course, going to work in an open-science culture organization does not mean you simply step up to a day of joy. The labors of science are infinite. The disappointments and the setbacks are legendary. All the joys offered in science are earned. "The joy of science lies in pondering the magnificent and seeking answers to the unknown. Indeed, Stephen Hawking's advice to 'Look up at the stars and not down at your feet ... Be curious'... is not far from what other scientists have noticed drives many scientific discoveries: the experience of awe"(McPhetres 2019).

One of the goals for culture change in open science needs to be an acknowledgement of the role of intrinsic motivations, and a cultural devaluing of the external, often perverse (Binswanger 2015), incentives that create so many conflicts of interest today in science. "We call for a cultural change in which scientists rediscover what drew them to science in the first place. In the end, it is not the number of high-impact-factor papers, prizes, or grant dollars that matters most, but the joys of discovery and the innumerable contributions both large and small that one makes through contact with other scientists"(Casadevall and Fang 2012). Like other psychosocial skills, joy increases across time when you work at it. Fun, 
however, can erupt at any moment when two or more scientists get into a conversation (or a "cerebration" (Asimov; Accessed May 1, 2020)) about their research.

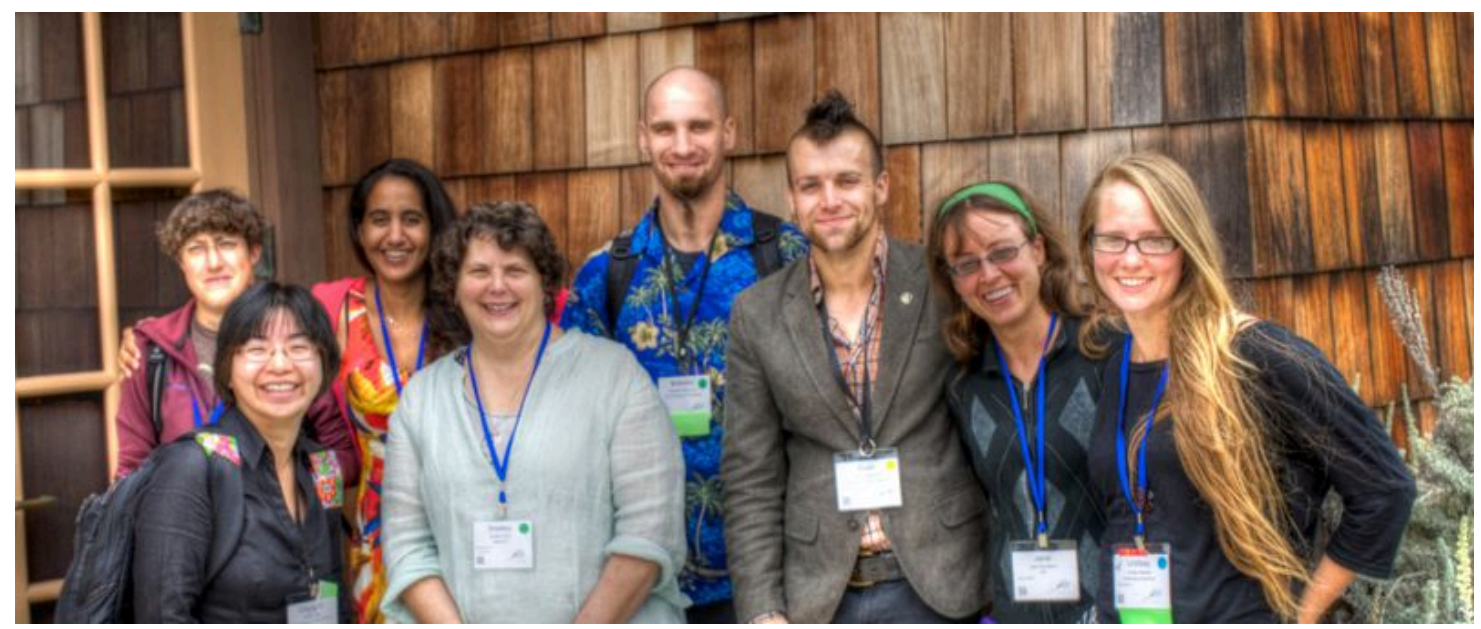

\section{Are you having fun yet?}

"For best purposes [for creativity in a group], there should be a feeling of informality. Joviality, the use of first names, joking, relaxed kidding are, I think, of the essence-not in themselves, but because they encourage a willingness to be involved in the folly of creativeness. For this purpose I think a meeting in someone's home or over a dinner table at some restaurant is perhaps more useful than one in a conference room" (Asimov; Accessed June 8, 2020).

\section{Science play is serious play}

There is a whole literature on "play" and and its valuable role in creativity and imagination from childhood to the board room. Linder, Roos, and Victor (Working Paper 2001; Accessed June 4,2020 ) do a good job of surveying this literature. Their Institute proposed "serious play" as the foundation for strategic thinking. Thomas and Brown (2007) look more specifically at the collective knowing that multiplayer games produce, and how this might inform new theories of learning. Csikszentmihalyi's work on autotelic, optimal experiences (what he calls "flow") describes how play-in a wide range of environments, including the workplace-offers its own very important incentives and rewards. His 2004 TED talk is a good starting place. Caron (2017) looks at how the study of society can be based on the 
cultures of games, as emergent, open-ended, strategic play. Here is it helpful to not contrast "play" with "serious". Play can be terminally serious; look at sword-play. Play can be artistically virtuosic: as in word-play. Consider open science as full of play: data-play, theory-play, methods-play. Remember too, play is fun. That is a bonus.

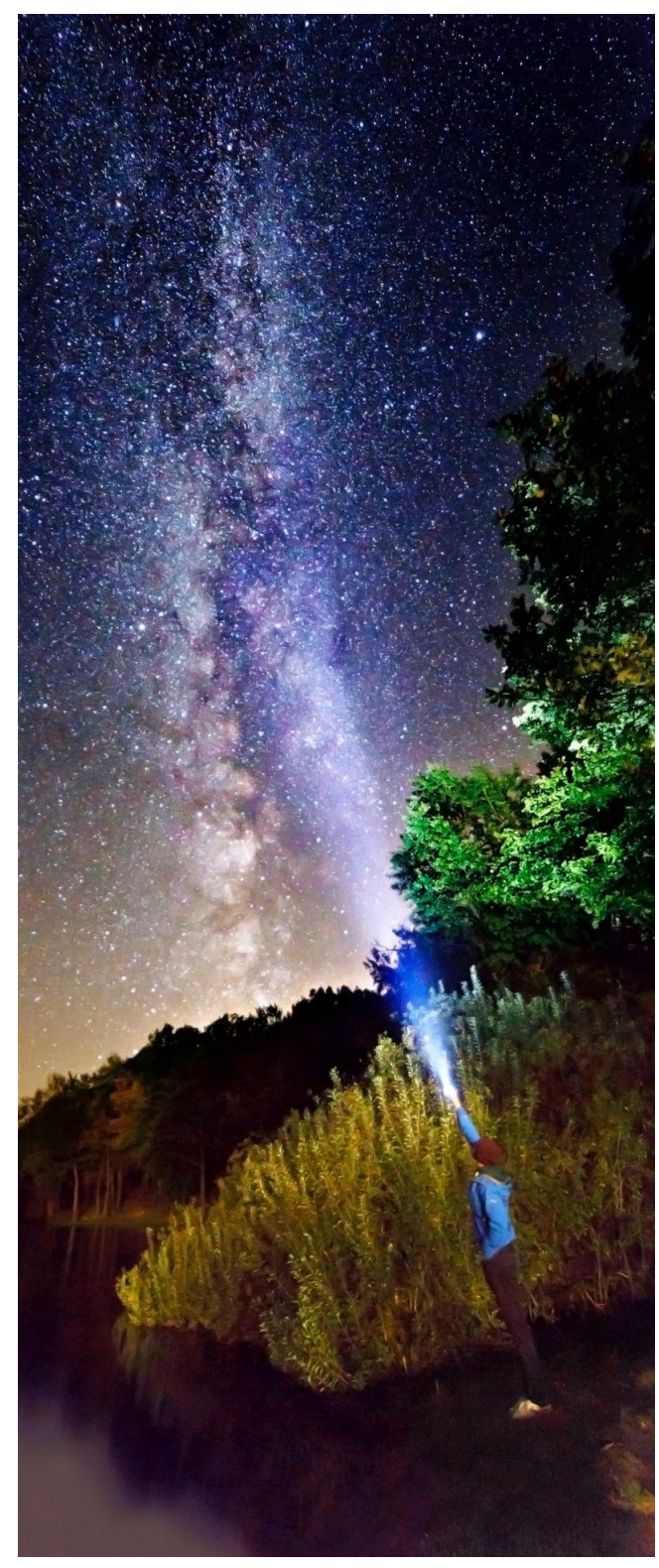

\section{Your love of science might have started early}

Open-science-culture governed organizations can intentionally, and reflexively promote events where creative folly-the play of intellection-is more likely to occur on a more regular basis. When they abandon the finite games of metrics-turned-into-goals, these organizations will find new spaces and time for serious play. Fun is guaranteed as a first-order outcome, together with more creative imagination, an increase of shared knowing, and the likelihood of better problem solving. Asimov (above) noted that bosses and funders might not fully appreciate the level of fun involved in events of group creativity. He suggested that participants be given "sinecure" tasks - to write a white paper, say, or a final reportsomething perfunctory to keep the funders happy and the bosses complacent. Never confuse these tasks with the real work, and the serious play of scientific conversations.

It's clear from the literature that talking about play and fun in the workplace surfaces a tension between two models of academic work. The first model tells us (and our funders) that work needs to be endured. And for it to be endurable in a meaningful fashion, it must be difficult and arduous. No fun allowed.

Neoliberal managerial practices serve here to ratchet up demands and metrics to be sure that next year, or next week, you will need to work harder than today. So, buckle up and buckle down, because somewhere else, others are working harder than you are, and you will be left behind, unfunded, and tenure-less. 
The second model tells us that each scientist has-through many years of learning and striving-earned the right to join the infinite play of science, which has no clock, and runs on shared knowing and ubiquitous doubt. This work is no less arduous. However, there is also laughter and joy, and a love for the process of doing science and for the object of study. "A love of knowledge, the most valuable resource in Universities, is being squandered by policies designed for the market place" (Rowland 2008). Open science culture change can move your team and your organization from the first model to the second one. This Handbook will help.

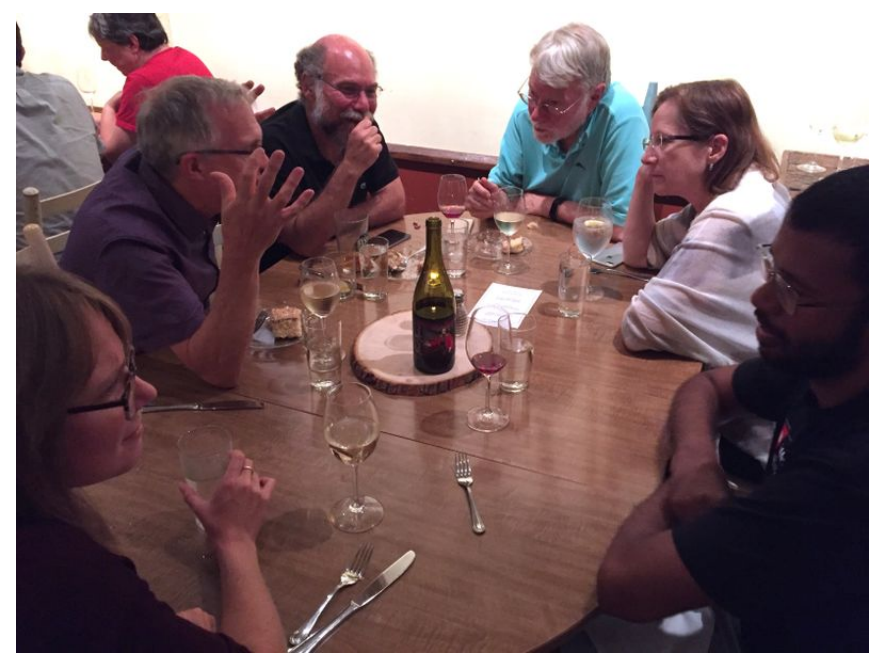

'opens'; DOI:10.31235/osf.io/2kxq8.).
Cerebrations are the events of science

You don't love science because it's fun. You have fun doing what you love. It's called science.

"The lesson here for Open

Scholarship may be that an inherent personal love of science and discovery must be nourished..., and communities that can affect the principles of Open Scholarship must also be cultivated around this" (Tennant, et al. 2020 A tale of two

In the end, you cannot talk about open science without adding how this enables new/old practices that show the love of science and the love of nature through science. And you can't really talk about changing cultures in your workplace without asking the question: does this workplace nurture the love that its workers might find, the joy they can feel and share, and the fun they can generate through their work and with their time here? As Roland (2008) notes: "[I]t is somewhat ironic if academics consider a term such as a love of knowledge-or 'intellectual love'-should not be taken seriously. It is strange that a phrase such as 'the delivery of learning outcomes' is taken to be serious and meaningful, but not 'inspiring a love of learning'. Has talk of such love no place in the language in which academics write about their work?"

One of the cultural aspects of open science that deserves more conversation is the replacement of external incentives with those internal incentives that have long been a part of science, but which have been demoted and shunted in the pursuit of finite games and the 
logic of competition. The love of science is a lifetime affair. It probably started for you in childhood. "As researchers we sometimes need to be reminded that we are contributing to an astonishing human effort, which transcends an individual's lifetime (Frith 2019). It is a big "why" for those career choices you've made. It also promotes trustful, caring relationships with other scientists. There is no room for: "I love science, it's scientists I can't stand" (See: Kindness, Culture, and Caring). A love of science also opens up an avenue for "slow science." "We need to engage at every level to accomplish a reconceptualization of university time. Creating spaces for new modes of scholarship and intentional communities helps us to move from individually-focused solutions to solutions with potential to create institutional and structural changes that nourish and support slow scholarship" (Mountz et al. 2015).

\section{It is time to slow down and smell the science}

"Science needs time to think. Science needs time to read, and time to fail. Science does not always know what it might be at right now. Science develops unsteadily, with jerky moves and unpredictable leaps forward-at the same time, however, it creeps about on a very slow time scale, for which there must be room and to which justice must be done."

From The Slow Science Manifesto (2010).

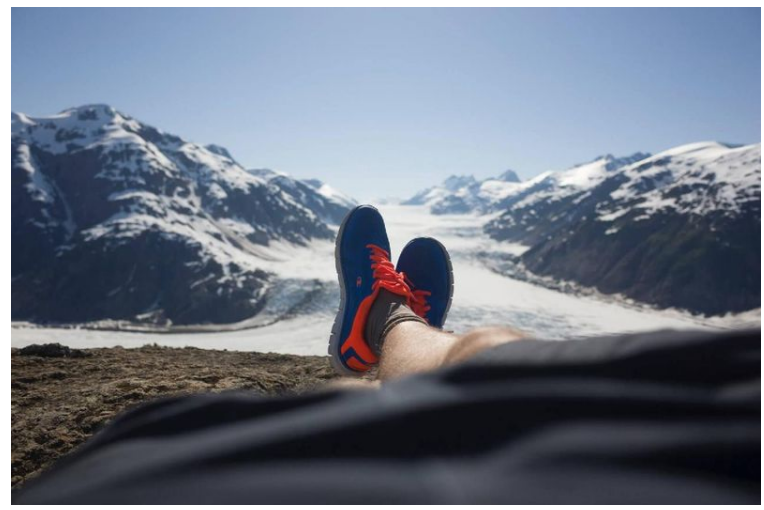

Sometimes slow is just about right

Open science is not only slow science, indeed, open science looks to accelerate knowledge sharing, but it does foster slow science, through conversations and collegiality, as a part of the future of how science is done. "This slowing down represents both a commitment to good scholarship, teaching, and service and a collective feminist ethics of care that challenges the accelerated time and elitism of the neoliberal university" (Mountz, et al. 2015). 
Open science is all about collaboration: "[I]n order for collaboration to work well, it emerges locally in conversations between people......Collaboration is about thinking together. And undertaken in that spirit, collaboration can allow us to challenge neoliberal models of higher education and the remasculinization of the academy" (Berg and Seeber 2016).

What if each scientist is limited to one published paper a year (with unlimited preprints, blogs, etc.)? What if each scientist can only receive three external grants in their careerand their home organization was responsible to work with funders and the public to increase the general, sustained, in-house support for science research? What if teaching the love of science were a large part of career advancement? The cultures of open science will foster emergent practices that can move the academy away from the current the neoliberal university model.

"Will Rogers used to say that people don't so much fall in love as step in it. I think the same may often be said of science. Even those who know from their third birthday that they will be a scientist can't tell you precisely how they got to be doing exactly what they are doing. They try this or that, run into a professor or a graduate student who takes him or her under their wing and infects them with their mystery, and that's it" (Firestein 2012).

\section{Coda: I thought science was all about rationality}

You could argue that "rationality" is central to instrumentalist practices in science and dominates the explanatory prose of science reports. Useful it is, we will all admit, within its domain. No disagreement here. All of the arguments for precision and intellectual rigor, for "doing the math," and being grounded in the methods: these are a given. There is no antirational basis nor bias in open science. The real issue is where rationality fits into the larger practice of science. What are the limits of instrumentalism? Where does imagination and serendipity show up? When do explanations fail? "Indeed, the exclusive attachment to purpose, consistency and rationality may be inappropriate in organizational situations that actually require reason's 'non-rational' cousins, including impulse, intuition and lived bodily experience" (Jacobs and Statler ,2004. Working Paper; Accessed June 8, 2020). You can be coldly rational with your research strategies, logical with your data, rigorous with your methods and still be kind, caring, and ego-free with your team.

Scientists regularly probe beyond what they can currently explain, hoping to extend the limits of explain-ability. As Carse reminds us, there are other forms of writing better suited to some of these unknown domains: "Explanations settle issues, showing that matters must end as they have. Narratives raise issues, showing that matters do not end as they must but as they do. Explanation sets the need for further inquiry aside; narrative invites us to 
rethink what we thought we knew" (Carse 1987). In its infinite play, science goes beyond explanation and steps into narrative.

Background: descriptions of hyper-rationality in the state (and the academy) flow through "poststructuralist" social theories, and discussions about poststructuralist theories, in the late $20^{\text {th }}$ Century (Harvey 1989; Bhabha 1990; Best 1991; Best and Kellner 1997). Michel Foucault's lectures in the late 1970s (Foucault 2008) are a fountain of these descriptions. As an open scientist, you can make a quick note that you are not alone in seeking a better way to build teams, share knowledge, and collaborate with your peers. You knew this without ever reading Foucault. 


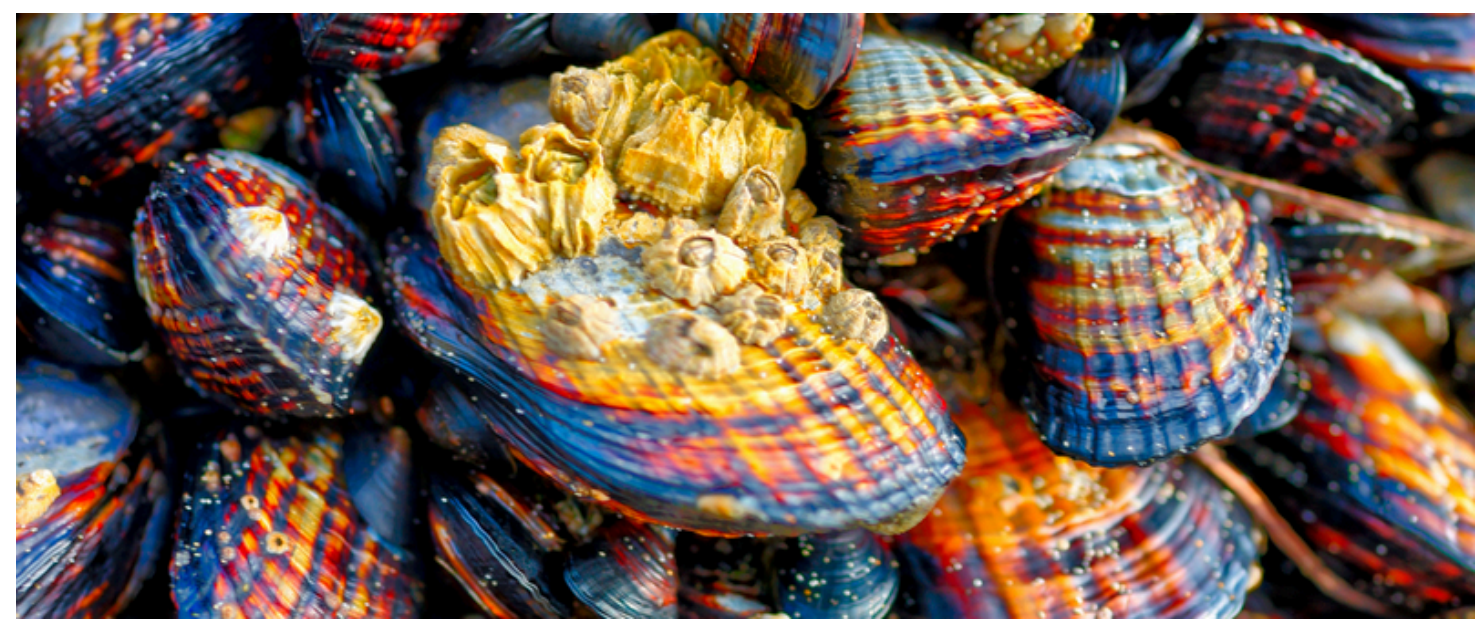

\section{Commons are the future homes for science}

\section{Open science builds scholarly commons (plural) across the planet}

Open science movements agree that the future depends on commons, even when the word is not present. NOTE: the photos in this section are of the California coast, which is in its entire length a public commons.

"We see a future in which scientific information and scholarly communication more generally become part of a global, universal and explicit network of knowledge; where every claim, hypothesis, argument-every significant element of the discourse-can be explicitly represented, along with supporting data, software, workflows, multimedia, external commentary, and information about provenance. In this world of networked knowledge objects, it would be clear how the entities and discourse components are related to each other, including relationships to previous scholarship; learning about a new topic means absorbing networks of information, not individually reading thousands of documents. Adding new elements of scholarly knowledge is achieved by adding nodes and relationships to this network. People could contribute to the network from a variety of perspectives; each contribution would be immediately accessible globally by others. Reviewing procedures, as well as reputation management mechanisms, would provide ways to evaluate and filter information."-FORCE11 Manifesto

"For the first time ever, the Internet now offers the chance to constitute a global and interactive representation of human knowledge, including cultural heritage 
and the guarantee of worldwide access"(Preface to the Berlin Declaration on Open Access to Knowledge in the Sciences and Humanities, 2003; Accessed April 13, 2020).

"Scholarly communication should expand the knowledge commons. Scientific knowledge is critical for the development of society. As scientific knowledge is intangible in nature, its use by one person does not preclude its use by another person. On the contrary, knowledge tends to grow when it is shared. Therefore, no barriers should be established to restrict the use and reuse of research results. Scientific knowledge should be a public good and as such part of the knowledge commons, in order to enable everyone in society to benefit from this knowledge."Principle \#12, Innovation, Vienna Principles

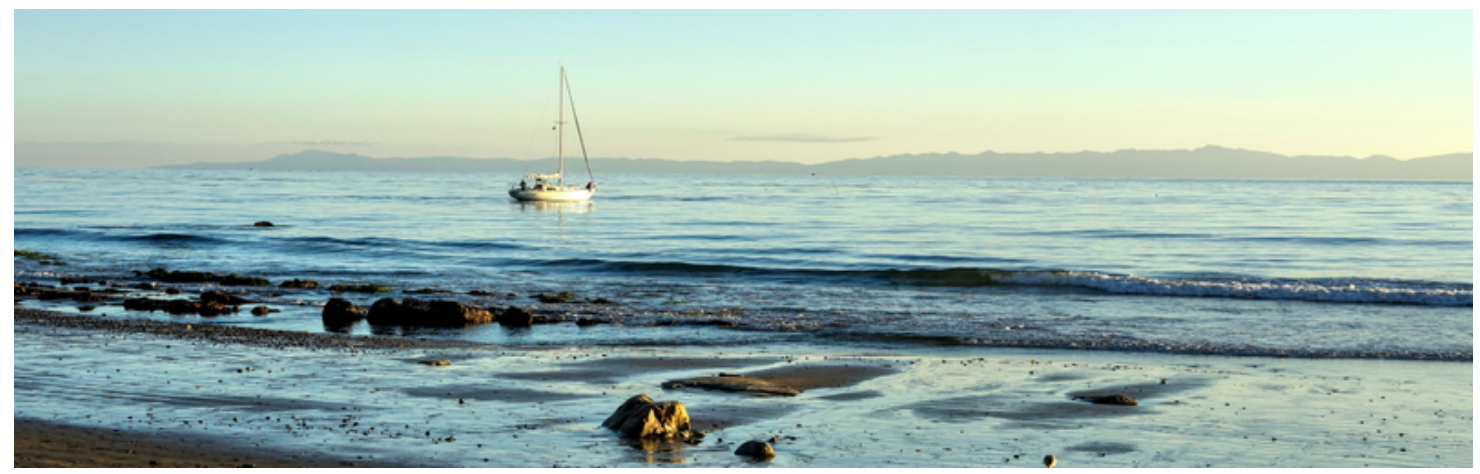

\section{Starting points toward commoning in open science}

In the following, "commons," "academy commons," "scholarly commons," and "science commons" refer to any commons created to house academic shared-pool resources-including networks set up for online conversations-and governed by a community that uses these for their research.

\section{Many thanks to the Alfred P. Sloan Foundation for supporting this work on the scholarly commons.}

- $\quad$ Science is intensely personal. Scientists are already engaged in their own struggle with the unknowns of nature through infinite play. Science-their intellectual disease-is fortunately incurable, and likely pandemic.

- Science is already social. Just in the US, several thousand workshops a year evidence the scientific need/desire to build collective knowledge. 
- Science is cultural. Self-governed science communities can use intentional cultural practices to help scientists prepare to work together in virtual organizations with shared norms and resources.

- Commoning communities open up arenas for online collaboration. Online conversation-driven collectives supported by virtual communities on internet platforms can replace expensive in-person workshops and massive annual meetings, and enable scientists to share knowledge and solve problems today across the globe.

- These communities need to consider themselves as commons to replace institutions that have been twisted by the three dimensions of external goods and influence (hierarchy, intellectual property, and neoliberal economics). Commons can address the many intellectual property wrongs that plague the academy today.

- Each commons needs to work locally, attuned to its local situation within science domains and academic institutions.

- The academy needs to harness the internet and technology platforms to knit together localized science/data commons into a global web of open shared resources and collective intelligence.

\section{Scholarly commons are...}

Intentional communities (plural) formed around the shared use of open scholarly resources (a type of common-pool resource). Commoners work together as a community to optimize the use of the open resources they share. Scholarly commons are resource-near communities. They have an immediate and professional stake in the open resources they need to use for their research. The whole community assumes a stewardship role toward these resources. These groups are self-defining and self-governing, each with their own emergent rules.

Since scholarly commons are usually built upon open public resources, anybody on the planet can access them. When these are digital resources, they are not diminished by overuse. However, these resources cannot be sustained without the commons, or some other economy. These commons represent the social/cultural destination for any number of open-science efforts.

Scholarly commoners are...

Members of these intentional communities, with the freedoms and responsibilities that their communities provide and demand. Commoners work for the benefit of the whole community and for the sustainability of its open, shared scholarly resources. An individual 
commoner may belong to several commons. It is the role and the goal of commoners to help these open, shared resources flourish.

Membership is implicit in a commons, and represents an active agreement to respect and celebrate the shared principles of the group. Membership will also require some attention commitment to governance and service.

Scholarly commoning is...

The practice (and an attitude) that commoners bring to the scholarly commons. It begins with a logic of abundance, and depends on an active culture of sharing. Commoning is the activity to build and sustain the commons through shared practice (thanks to Cameron Neylon for this wording). Scholarly commoning is also imbued with an ethos of scholarship/science (however defined). Scholarly commoning informs how science can be accomplished through the use of open, shared resources (open ideas, open data, open software, open workflows, open-access publishing with open review, etc.) inside commons, instead of through other types of economies.

Let's now explore in some depth what commons look like and how they work toward "science done right."

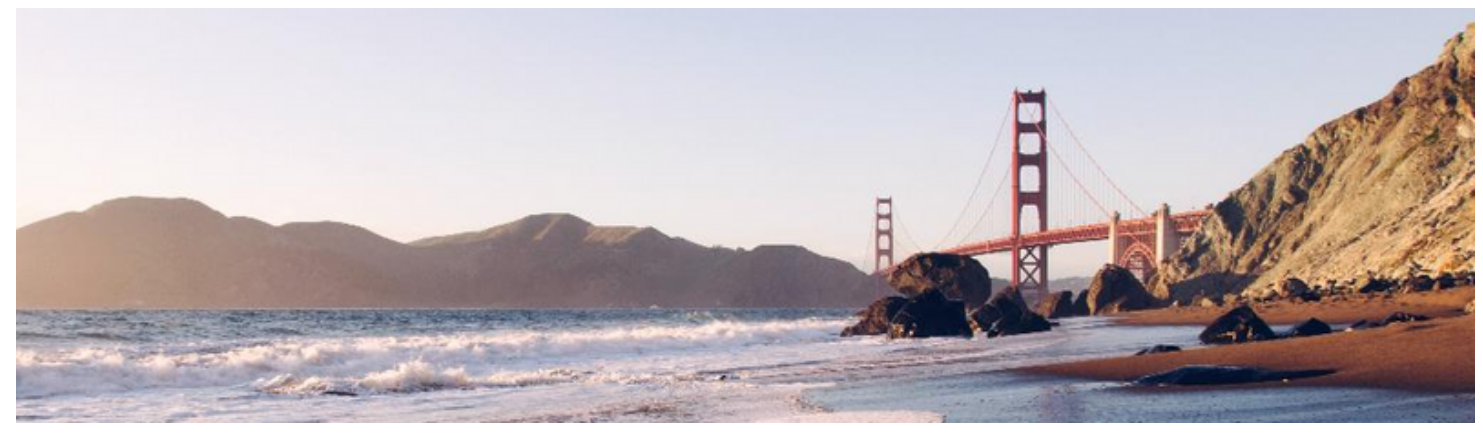

\section{Commons start with people: a community of commoners.}

To paraphrase Peter Linebaugh: "there is no commons without commoners."

Commoners contribute to and help govern their commons in many ways. They contribute a wide range of research-related objects and data; they ensure that these are sharable and discoverable through the use of appropriate metadata; they create "cerebration" events (See: Knowing and Conversation) to share ideas and scholarly objects, they collaborate in the development and use of appropriate standards and stewardship efforts; they acknowledge the efforts of others in their work; they promote the commons and commoning as a mode of scholarly effort. 
Because commons are owned and led by their communities, volunteers are given the responsibility to envision, build, and govern these as destinations for the future of open science and scholarship. All commoners will benefit from the impacts that their commons will make on the academy's research and communication capabilities. Volunteer leaders will also gain satisfaction that their time and efforts will grow these resources for the benefit of all and the advancement of knowledge.

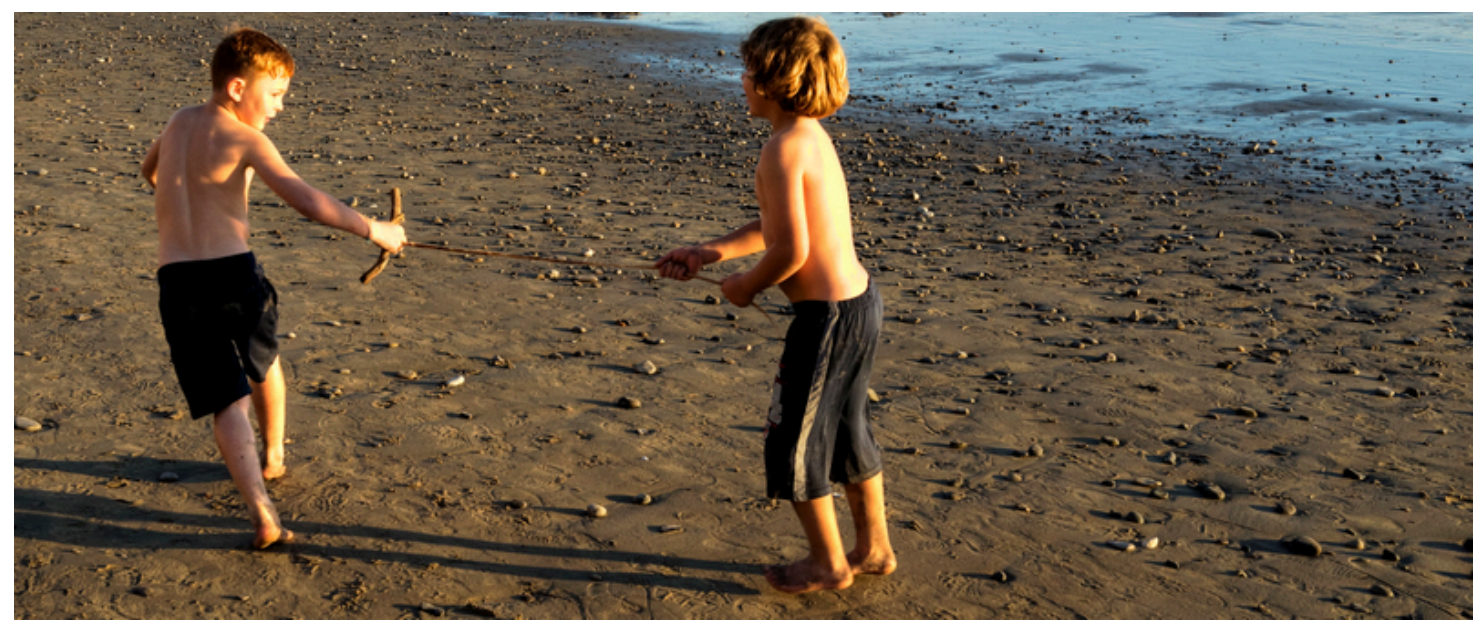

"Before every great idea is a crazy idea." Jono Bacon (2009)

"The world's cognitive surplus is so large that small changes can have huge ramifications in aggregate."

Clay Shirky (2010)

\section{These commons are open to all participants who accept their principles}

Commons can support a diversity of skills and knowledge without privileging any. All commoners will find a home for their knowledge and their interests. As a norm, participation in any scholarly commons is not restricted on the basis of accreditation, professional standing or reputation, or any other criteria except willingness to contribute and uphold the principles of the commons. Content and behavior are the only criteria for moderation within a commons.

Commons are intellectual "rooms" (See: Science happens elsewhere) that value active sharing and collaboration. Commoners serve these requirements in different ways across the spectrum of occupations and career paths. A commons does not require a specific volume or genre of contribution, a particular professional, educational, or social background, affiliation, certification, or status. 
The reach of the global commons network is not restricted to participants from any single sector or region. This network provides a home for the work of full professors, citizenscientists, entrepreneurs, and bloggers. It recognizes the comment, the scholarly monograph, the dataset, the discussion, and the commercial product or service. It provides a home and recognition for programmers, statisticians, bench scientists, and literary critics. It welcomes the most narrowly focused specialist work and the broadest popularization. Above all, it encourages commoners to collaborate and share their specializations and interests.

Each commoner gets more value than they give as they grow their scholarly commons (note: any scholar may belong to more than one of these). The return on investment (ROI) for the commoner demonstrates how a commons as a whole is more valuable than any of its pieces. One part of this equation is due to the "power of pull", which amplifies the value of participation, and also the utility of each object being shared in the network.

Commons are self-identified by interests, disciplines, experiences, data sources and uses, and research goals. Commoners across the planet will be linked as their local commons builds networks with other commons to expand the "room" they share to animate their conversations and creativity.

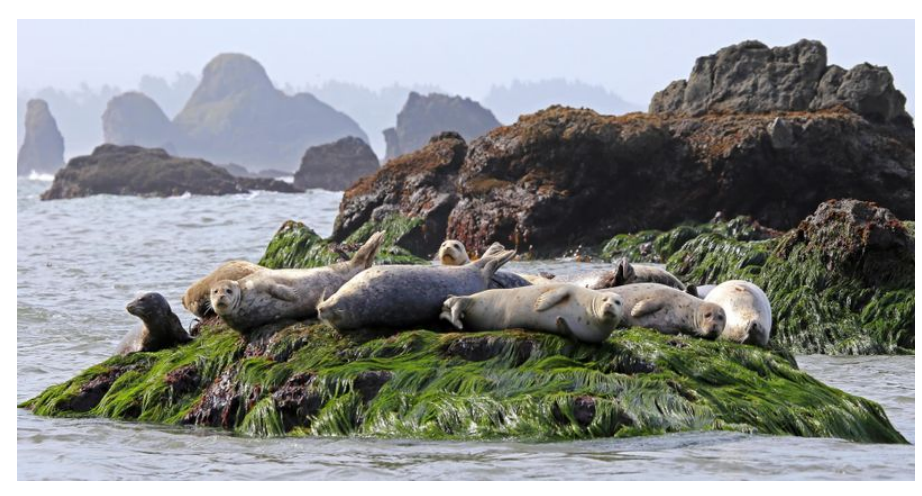

"Definitions belong to the definers, not the defined" Toni Morrison.

\section{Science commons welcome and encourage participants of all backgrounds}

Fierce equality means that every commons welcomes and encourages participants of all genders, social, regional, ethnic, linguistic, and disciplinary backgrounds. It also recognizes that disagreement is an inherent part of research communication, including disagreement as to fundamental principles and theories. 
A commons is an ecosystem that is defined by the interactions of each and every commoner who participates in jointly building and governing it. Just like every other ecosystem, a commons cannot be a monoculture; instead, it needs diversity in order to survive and thrive. While many scholarly disciplines differ in their culture of how to generate, treat and store their scholarly objects, their commons must be open to all of them.

In a similar way, scholarly commons can not only rely on the expertise of scholars employed at top-tier research universities. Instead, they need to be open and accessible to commoners that don't fit the academic stereotypes, or indeed never were in academia. Creating scholarly objects and performing scholarly activities is not limited to the core academic scholarly community. This means that commons must be open to diverse research questions and answers, including those proposed by non-professionals.

Through its self-governance, a commons uses vigilance with regard to hidden and structural biases and impediments and humility and open-mindedness with regard to the life-experiences of others. Because a commons is a shared agreement, the onus for ensuring equality and diversity of access is on the commoners themselves.

As commoners build self-governance, they should consider statements on inclusivity and language policy, because these encourage critical reflection on structural impediments. Exclusion of participation based primarily on formal degrees and academic rank is discouraged. When such criteria are used, alternative routes to participation should be provided.

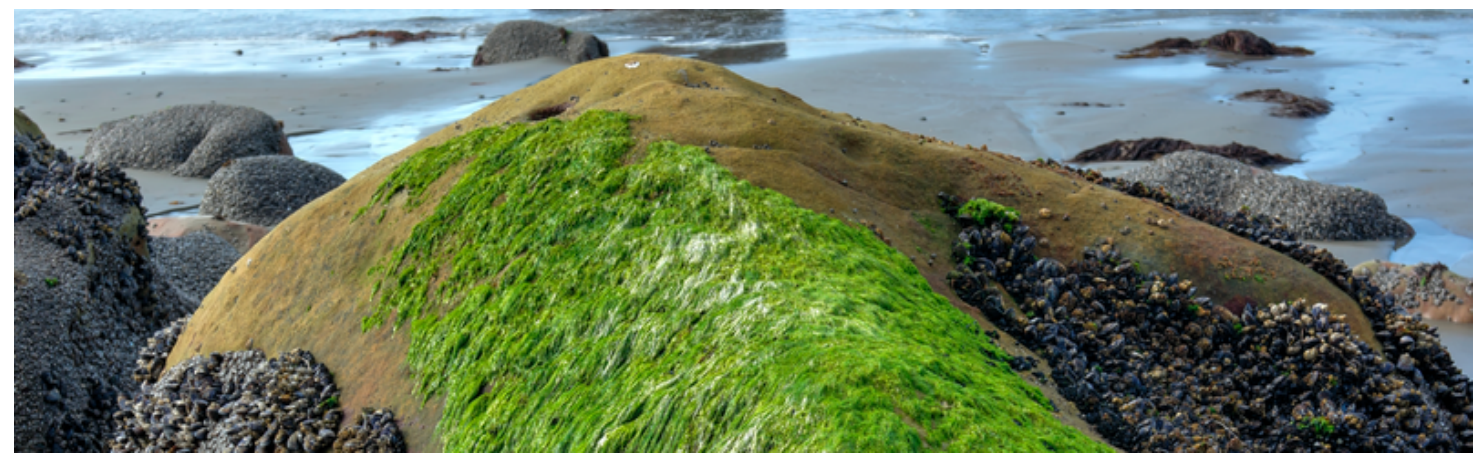

\section{Fierce Equality among objects for the pooled resource collection}

Commons accept all contributed objects that adhere to their guidelines on an equal basis regardless of form

In order to improve the breadth and pace of knowledge generation, a commons will accept any contributed object that adheres to its guidelines. Because commons are grounded by a 


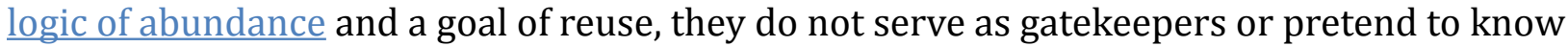
the ultimate knowledge-value of any of their shared objects.

This means that there is no test of value, impact, significance, relevance, or endorsement that can be used to determine what belongs within a commons. Blog postings are as eligible as scholarly monographs. Highly cited papers are as welcome as preprints. Groundbreaking studies are as welcome as replication studies.

Once an object is in a commons, it is available for additional services. For example, commons services could be implemented to help commoners search for objects. Early versions of objects can be peer-reviewed. Objects can earn citations. Objects may be further curated or aggregated into collections by other commoners based on their expertise. Data objects can be evaluated for provenance and various qualities that improve their use and reuse.

Some services will not be provided inside a commons. For example, others in the academy may want to add metrics or rankings to objects in a commons. Commons have no objection to these services, however all forms of metrics should be built on transparent and open standards so that they may be reproduced and understood. Rankings will be made external to a commons and will not be housed inside the commons.

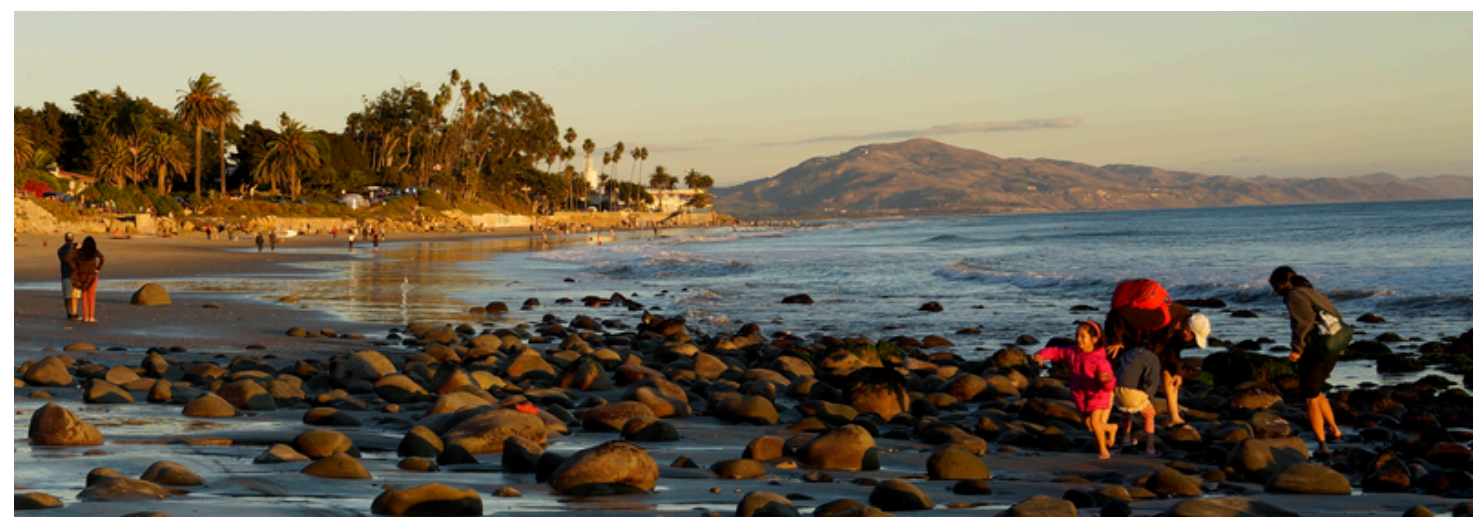

John Wilbanks: “Going back to the beginning of science: it used to belong to all of us."

Smaldino and McElreath (2016): "when a measure becomes a target, it ceases to be a good measure."

"We reaffirm the principle that only the intrinsic merit of the work, and not the title of the journal in which a candidate's work is published, will be considered in appointments, promotions, merit awards or grants."-Bethesda Statement on Open Access Publishing, 2003; Accessed April 9, 2020. 
"Do not use journal-based metrics, such as Journal Impact Factors, as a surrogate measure of the quality of individual research articles, to assess an individual scientist's contributions, or in hiring, promotion, or funding decisions."-DORA; Accessed April 9, 2020.

\section{Science commons have no intrinsic hierarchies, rankings, or reward systems}

All participants and all research objects that conform to the principles of the commons are equally appropriate and available for dissemination and reuse. Attribution systems and formats are driven by the demands of transparency and the intrinsic nature of research, rather than the requirements of any reward system. Intellectual humility (See: Kindness, Culture, and Caring) is expected in these commons as internal good and a norm for science, crowding out bullshit prestige.

All contributors are acknowledged on an equal basis (meaning there is no intrinsic difference between authorial and other acknowledgements); all forms of dissemination are accepted on an equal basis (meaning there is no hierarchy among genres or formats). Commoners are expected to match the form of dissemination to the needs of the research output rather than the demands of a reward system. None of this is compatible with systems that create hierarchies among types or forms of contribution or encourage dissemination in one format over another.

The fundamental premises of a science commons are incompatible with "scooping", because the commons does not require these ideas to be new or unique as a condition of entry, even though the commons tracks when and where ideas and objects enter the commons.

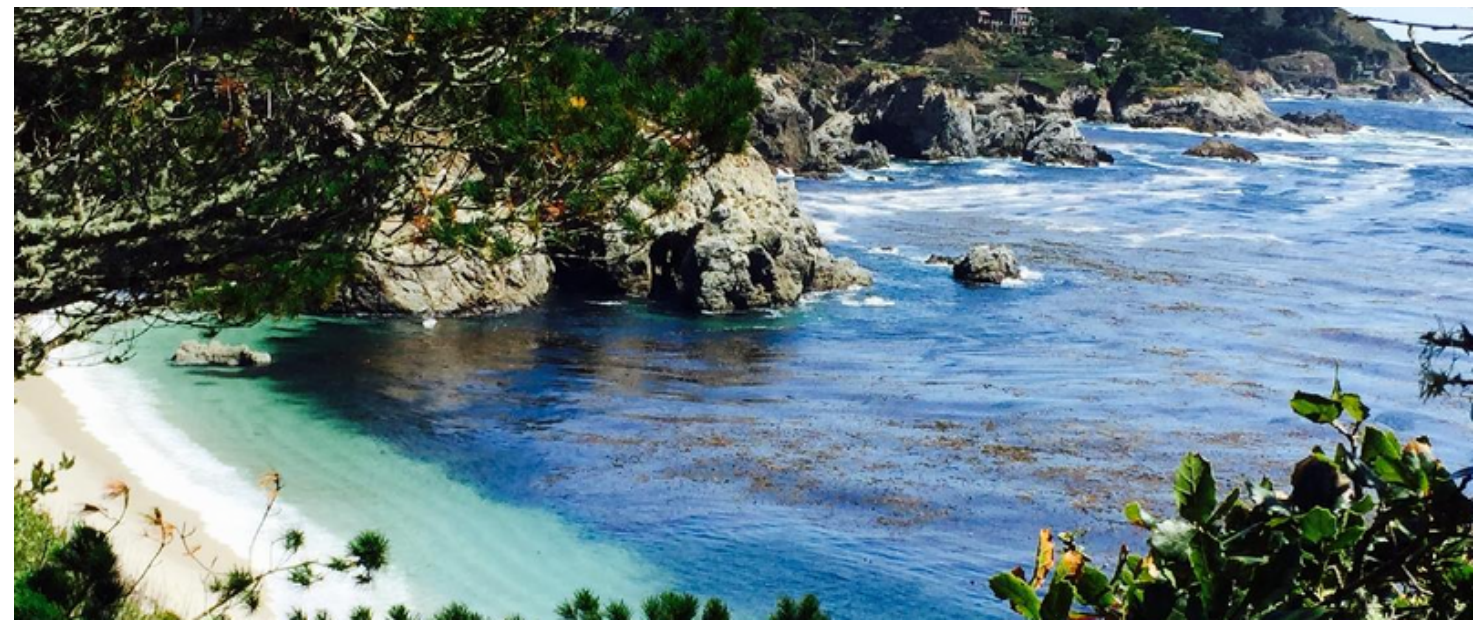


"...[I]f we could solve the problem of open access within the university-that is to say, prove that the economic equation of doing research, reviewing it, and making it freely available for everyone works, then we could prove that the tyranny of the margin need not operate everywhere." (Kelty 2014)

"Our mission of disseminating knowledge is only half complete if the information is not made widely and readily available to society."-Berlin Declaration on Open Access to Knowledge in the Sciences and Humanities; Accessed April 9, 2020.

"Computer analysis of content in all formats, that is content mining, enables access to undiscovered public knowledge and provides important insights across every aspect of our economic, social and cultural life."-Hague Declaration on Knowledge Discovery in the Digital Age; Accessed April 9, 2020.

"When intellectual property law allows content to be read and analysed manually by humans but not by their machines, it has failed its original purposes."Declaration on Knowledge Discovery in the Digital Age; Accessed April 9, 2020.

\section{A commons is open by default, with a culture of demand sharing}

Openness for demand sharing is the core norm for a commons. Its resources are intentionally and reflexively open and entirely free to use, read, reuse, and remix by humans and machines, unless there is a compelling reason to restrict access, e.g., personal health information. Scholarly commoning starts with openness as a norm, and supports activities that explore open scholarship fully. Demand sharing is the main activity in a commons.

Commons can use standards and guidelines developed by other organizations (e.g., OKFN Open Definition, Budapest Open Access Initiative guidelines, and the Open Source Initiative definition) to inform their core definition of open content and access. Openness will be reinforced through the use of licenses that support the sharing of outcomes, such as knowledge gained by mining commons resources, research undertaken using commons resources, and software derived from commons code.

Commons will support a variety of open licenses. In their daily practice, commoners heed the requirements of these licenses and add their own content through them. Open includes promoting machine access to resources and metadata. Openness includes the right to deposit as well as to access, read, analyze, cite, quote, and mine. Where privacy is important to protect the rights of data providers or subjects, commons will make best-practice efforts to secure these data.

Demand sharing necessitates a radical rethink by stakeholders in their relationship with research assets produced by 'their' researchers, using 'their' funding, published within 'their' publications. The openness of commons allows the development of external services 
that can be more closed, proprietary, or involve ranking and selection: e.g. aggregation and indexing services, as long as they do not devalue the commons.

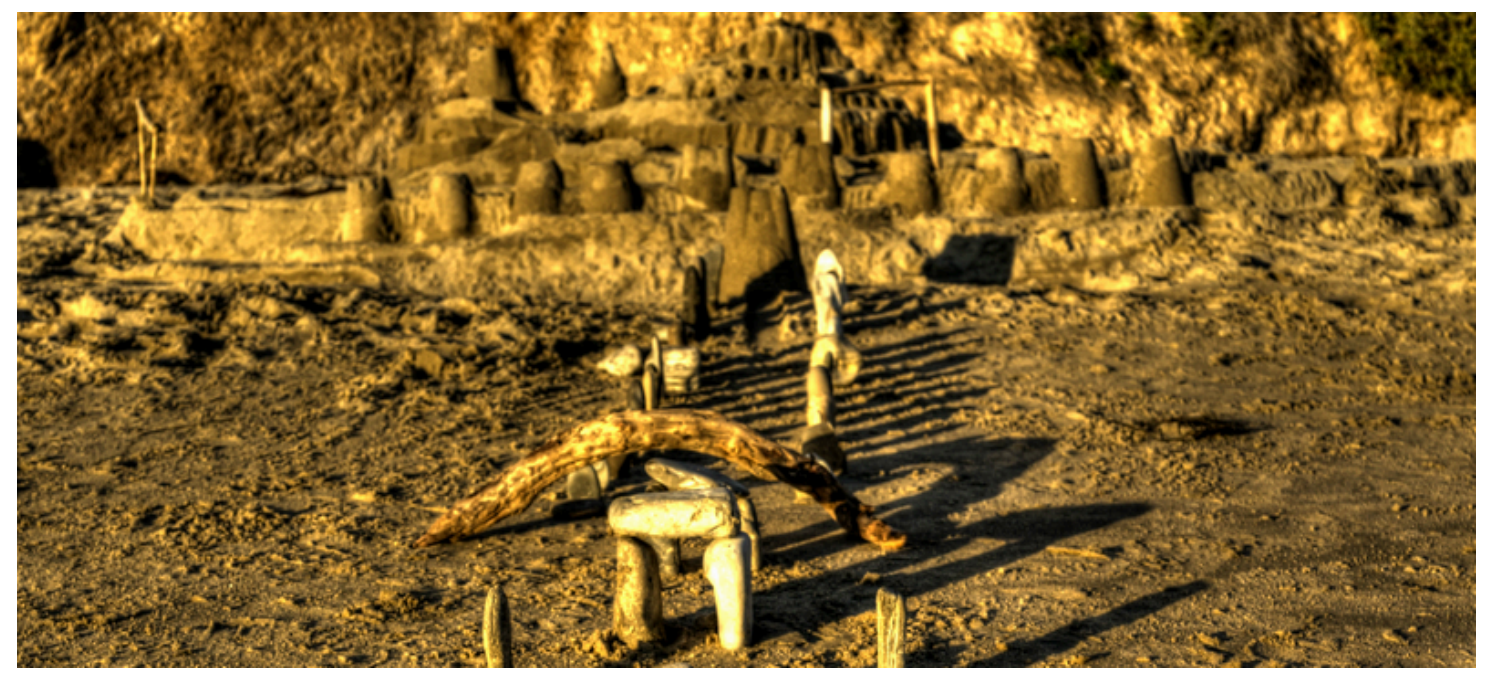

"In many instances IPRs [intellectual property rights] appear to be privatizing and commoditizing-“enclosing"-socially useful knowledge that, if widely shared, could result in more affordable and accessible medicines, scientific research, educational resources and climate technologies. In recognition of this reality, EU policy ought to empirically examine whether existing policies are sanctioning severe opportunity costs. By recognizing contemporary technological and economic realities, EU policies could unleash moves towards more affordable health systems, wider uptake of green technologies, a more open, participatory creative culture, and more responsive democratic governance" (The EU and the Commons 2015; Accessed April 12, 2020).

\section{Any person, organization or other entity can make scholarly work public.}

As long as the criteria for open sharing are met-as determined by each commons through their governance-making a work publicly available is considered publishing a work. Additional requirements to add value for reuse (metadata, provenance, reproducibility, etc.) increase in value in a demand-sharing science culture, where outputs can be mined, mixed, and repurposed. Any person, organization or other entity (including publishing companies or entities that currently act as such, e.g. scholarly societies) will be welcomed by commons for providing services that help the publication, preservation, dissemination and assessment of scholarly work, as long as these services and the outputs they produce comply with the demand-sharing principles of the commons. 
As commons content is stewarded by commoners within the commons, exclusive rights to this cannot be sold within or without the commons. Fees for additional services designed to maintain shared resource availability can be managed within the commons. Fees for access outside of the commons, and outside of the larger networked commons endeavor can be used to fund commons expenses, but cannot restrict access to content inside the commons. Operational principles for those who provide infrastructure for a global scholarly commons network are laid out in Bilder et al (2015).

Science commons explicitly reject the current model of publishing scholarly works which emphasizes the release of works only when they have undergone the peer review process. In a commons, a published work may be a version of a work that gets subsequently refined, similar to the way that open source software is released and then refined. Additional layers of curation, peer review and editing will be performed on works as needed for purpose. Note that these comments, discussions, annotations are themselves scholarly objects.

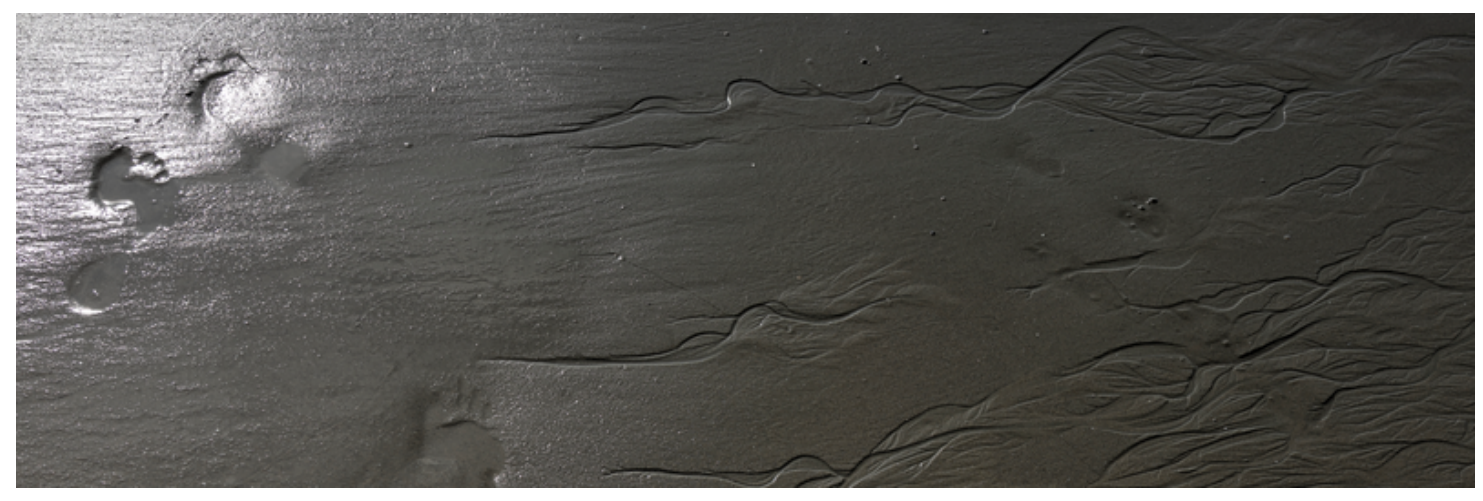

"Building a small ecosystem of capped returns is all well and good, but it won't make much of a difference in the grand scheme of things. This idea has the most potential for impact if it becomes the new norm and displaces indefinite returns significantly - maybe entirely." P2P Wiki

"Effective institutions at all levels require continuous engagement, because they all unravel over time." (Benkler 2015)

"A country, after all, is not something you build as the pharaohs built the pyramids, and then leave standing there to defy eternity. A country is something that is built every day out of certain basic shared values." Pierre Trudeau.

"Communal values must be taught, and renewed, continuously." (Peter Linebaugh 2014) 


\section{Sustaining scholarly commons: There is global commitment and participation in long-term viability and preservation.}

A global community is needed to actively grow and sustain science commons in the long-term. The shared-pool resources of hundreds of commons across the planet will need, and should command public funding for maintenance and growth. Each state has a stake and role in preserving and promoting the active knowledge available through commons repositories hosted in their territories, for the benefit of all science.

Commons will not flourish without the participation of commoners within the commons and also as citizens of their polities, promoting commons norms and values across societies. Commoning as a feature of scholarly work needs to be taught at all levels, practiced in everyday work, discussed and improved through reflexive innovations, and celebrated across the globe.

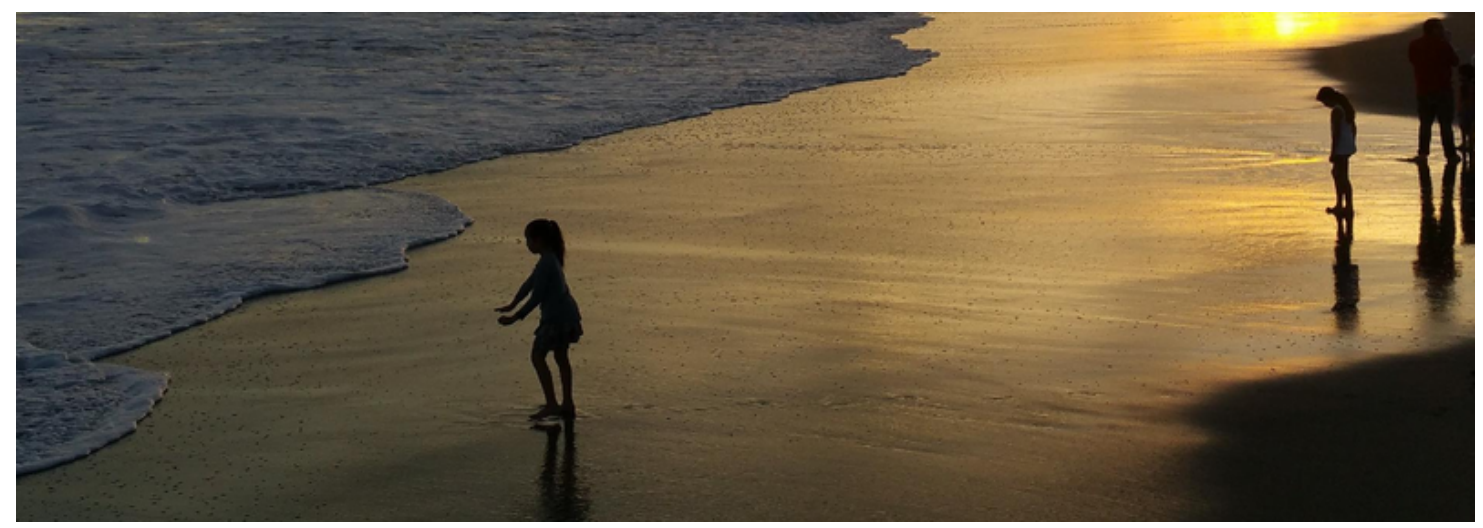

"An economics of abundance seek out these kinds of strategies of providing for our needs; it is not an economics that assumes that abundance exists, but one that analyzes modes of scarcity generation..., and that points out ways to counteract them" (Hoeschele 2010).

"It seems like if we could re-frame the way we think about these problems, and find new abstractions, new places to stand and see the issues we might be able to break through at least some of those that seem intractable today. How might we recognise the unexpected places where it is possible to create abundance?"

(Neylon 2015); Accessed April 9, 2020. 


\section{All activities and outputs that take place within commons have a permanent home in these commons and are available to the public.}

All content and services in scholarly commons will be publicly shared. All resources are openly available and may not be removed. There is, of course, a differential built into the amount of prior learning that enables various uses for commons resources. Mostly this is a built-in feature of the complexity of the research endeavor, and the extreme complexity and emergent qualities of the current state of research in any field. A chess club may be open to all, however, the skills needed to play well with the most advanced members become available mainly through long-time learning and practice. Similarly, optimal use of scholarly commons resources very often requires years of training/learning. See also: Against Exclusion: open is open to all.

Currently, academic research is surrounded and interpenetrated by an economic logic that manufactures scarcity as a means to grow arbitrary value and improve profit margins. The academy needs to grow its own digital economy. And for this, it needs to capture the value that researchers invest into it. One part of this exchange value will come from the expansion of internet-enabled services, another from the increase of its digital resources, and a third from the contributions of scholarly talent and funding sources.

While it is tempting to try and capture more value for pooled resources inside a commons by creating a differential use license that restricts use outside of the commons, there are more effective means available for this purpose. Commons can create or participate in civic trusts, set up like land trusts are today, which hold key aspects of the property rights for commons resources, and can negotiate with other commons and with external interests for the use of these resources (See: McDonald 2015; Accessed April 10, 2020). Cultural practices that support demand sharing within commons can also be effective in reducing behaviors such as extracting resources from the commons or seeking advantages by working with external interests.

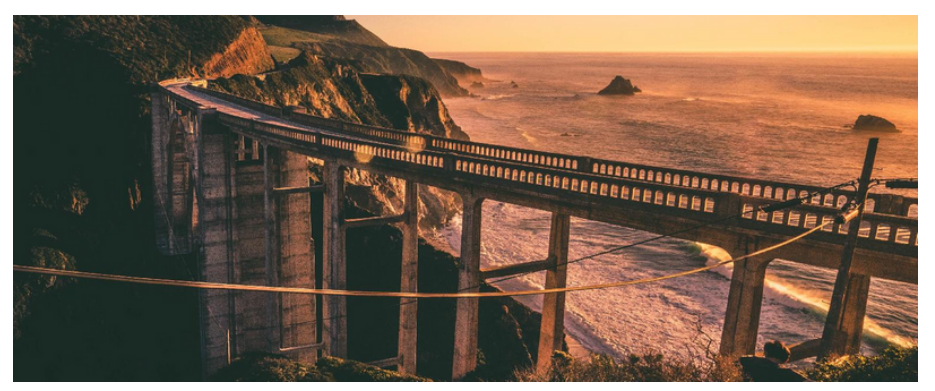

"With 'subtractive' resources such as fisheries, for instance, one person's use reduces the benefits available to another. High subtractability is usually a key 
characteristic of common-pool resources. Most types of knowledge have, on the other hand, traditionally been relatively nonsubtractive. In fact, the more people who share useful knowledge, the greater the common good" (Hess and Ostrom 2009).

\section{Demand Sharing means that the use of commons resources cannot devalue these}

The logic of scholarly commons starts with the notion of abundance. One mission of scientific commons is to manage a full range of science objects, without needing to reject some because of an arbitrary constraint on capacity or a responsibility to judge their value. The aim then is to maximize the usefulness and usage of these objects by supporting discoverability, mining, sharing, and reuse. Unlike natural resources (a fishery, a forest, etc.) the digital objects in scholarly communication are anti-rivalrous. Their use by one member does not devalue their use by another. Overconsumption is not a concern. The optimal state for the global network of scientific commons is one that supports as much consumption of their resources as is technically possible (See: Abundance).

In any one commons, the active sharing of resources, and the added opportunities for creative conversations and "cerebrations," produces a great variety of outcomes and records, and new generations of results that had been enabled by the commons, and that get returned to start new cycles of knowledge building and knowing. Every commons anchors deeply into shared infinite play. Each commons reaches out to other commons to expand the horizon of the infinite play of science. In some ways, each commons is selfsustaining: a crucible of activity and joy that fuels itself.

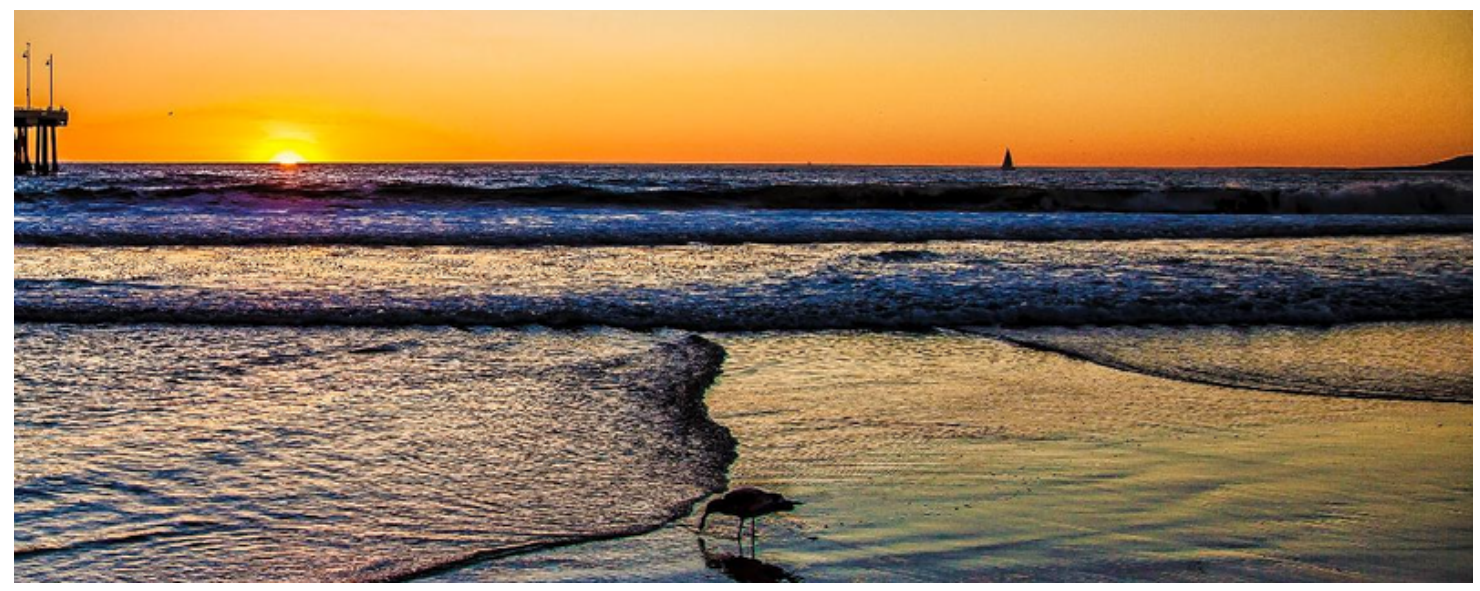

Do-ocracy: "Responsibilities attach to people who do the work, rather than elected or selected officials." P2P wiki. 


\section{There is an expectation of service by Commoners to support research and scholarship in the Commons.}

Commons will establish their own forms of do-ocracy. This is a generalizable feature of self governance for any scholarly commons. Leadership will be gathered from the edges, where new working groups will be building and expanding the collection and its services. Effective group leaders will find that their service opens up new doors for greater service (no good deed goes unpunished). A reputation for accomplishing significant work will form the basis for participation in leadership roles.

Scholarly commons build on a tradition of service; scientists have been gifting their research results to the republic of science for centuries now. The types of activities that constitute service are expected to be enlarged and their capacity for documentation enriched, e.g., participation in online conversation forums. As a general rule, individual scholars and teams will always receive more value from their commons than they contribute. This primary surplus of value is not just due to the network effects of commons, but also from the added opportunities for serendipitous interactions. The value proposition that each vibrant commons represents can be expressed explicitly on an individual, institutional, functional, disciplinary, national and global basis.

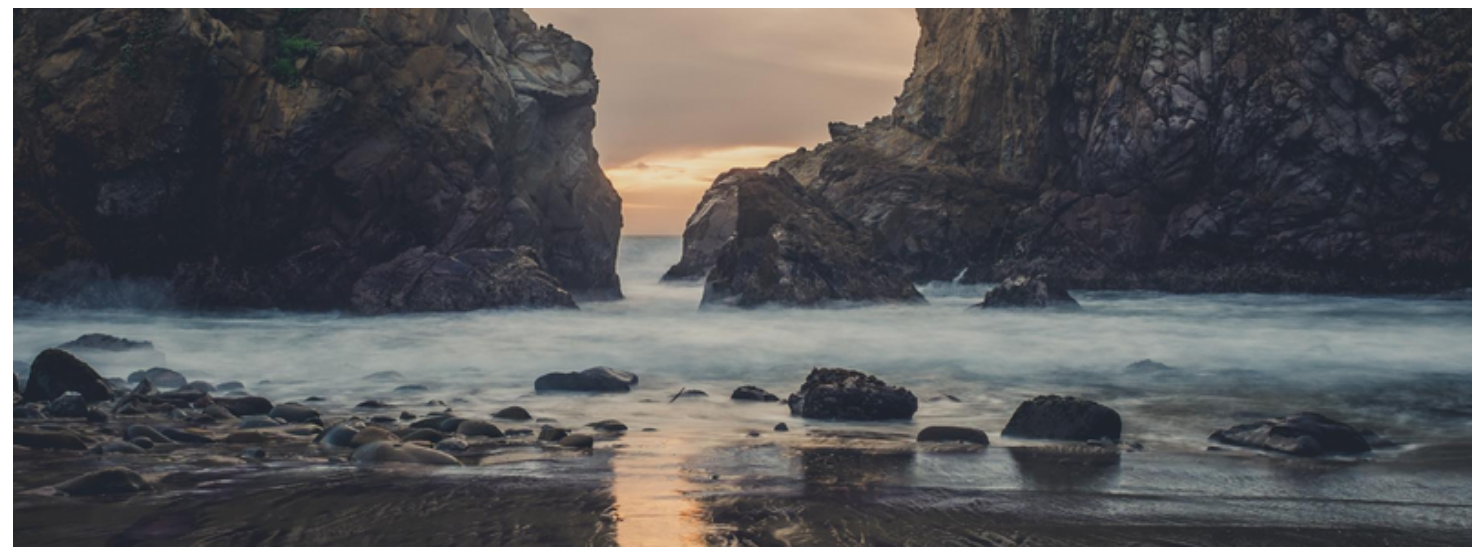

"Single loop organizations fix problems... Double loop organizations fix problems and fix the situations that caused the problems" (Shirky 2011; Accessed April 12, 2020).

"[P]rofessions have specific 'internal goods'. They include truthfulness, analytical skill, and buying into the professional's fiduciary duty to their client in the wider 
context of behaving with integrity to all. To acquire such internal goods of practice - or 'goods of excellence', as he subsequently termed them-MacIntyre [1984] argues that one must practice at least three virtues: justice, courage and honesty. When practising one's profession, one can't make up one's own facts. And a good argument is one that would persuade the best of one's colleagues, not just one's own side. Thus, just as Francis Bacon proposed-sublimely-regarding the growth of science, that we cannot command nature except by obeying it, so the professional must master and obey the imperatives of their discipline to gain access to the agency it offers. This idea of engaging with an external or objective order implies justice, which is secured only by allowing correctness within the practice to trump ego or power. This, in turn, implies equal treatment for equal merit within the terms of the practice" (Gruen; Accessed April 14, 2020).

\section{Commons become community-based value-generators for the work of their members.}

The way forward requires an effort that spans the entire practice of scholarship, from intellection to publication. Researchers face the task of redesigning the scholarly workflow, while they inject these new modes of doing research and publication into the broader academy. The life of a scholar is rigorous and difficult, but also includes opportunities for personal and collective fulfillment. As commons spread across the academy, these will generate local communities that do two important tasks for each member: the communities cascade collective meaning into scholarly practice at the team level, and they support cultures of caring and kindness, and trustful events and friendships. They hold shared, internal virtues (goods) as binding on their members. As MacIntyre (1984) reminds us: "[T] he essential function of the virtues is clear. Without them, without justice, courage and truthfulness, practices could not resist the corrupting power of institutions."

Virtues and normative practices in a commons are promoted to stimulate behaviors that support the production and dissemination of the best scholarship and science. They encourage respect for the principles of these commons, and they discourage behaviors and practices that inhibit participation. They apply to all stages of and participants in the research cycle. They respect and support non-standard research outputs (such as datasets, software, methods, null-results, ideas) and para-scholarly activity (e.g. leadership, community service, peer review, and adjudication).

Researchers across the globe will have wide variety of local issues to bring to their commons. The academy today is broken in various ways that reflect cultural issues locally. Each organization and discipline faces their own version of these dysfunctions. While all solutions are ultimately local, every commons creates helping practices that can be shared laterally across the planet. 


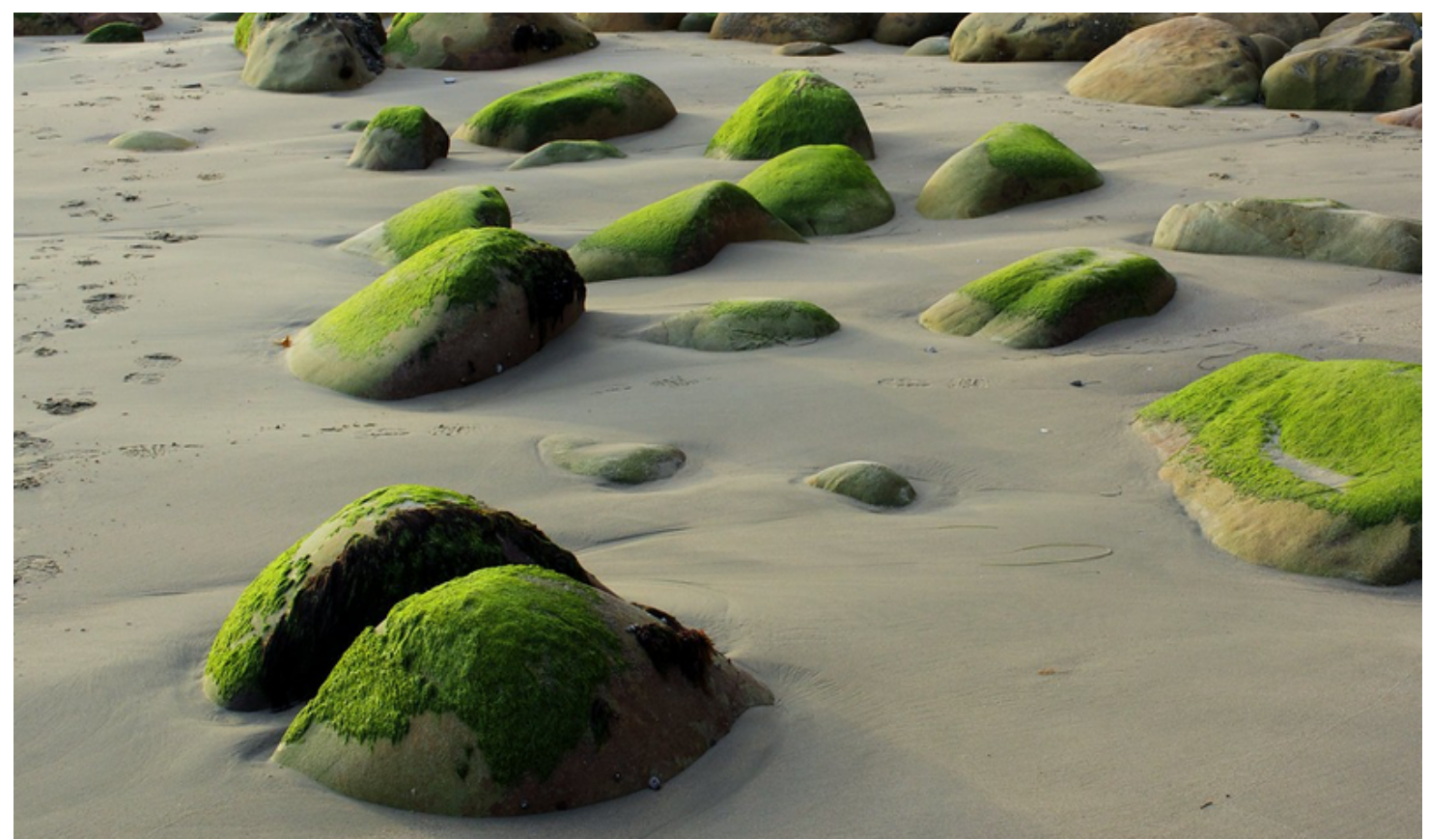

"What I call software collapse is what is more commonly referred to as software rot: the fact that software stops working eventually if is not actively maintained. The rot/maintenance metaphor is not appropriate in my opinion because it blames the phenomenon on the wrong part. Software does not disintegrate with time. It stops working because the foundations on which it was built start to move. This is more like an earthquake destroying a house than like fungi or bacteria transforming food, which is why I am trying out the term collapse" (Hinsen 2017: Accessed April 13, 2020).

"How do we ensure that the system is run "humbly", that it recognises it doesn't have a right to exist beyond the support it provides for the community and that it plans accordingly?" (Bilder et al 2015).

\section{Scholarly commons exist outside of specific technologies, funding sources, and business models}

Scholarly commons accommodate, facilitate, stimulate and adapt to any developments and technologies that promote their goals, and enable their practices. Because the needs of commoners and the means to meet these will be emergent, commons must remain nimble and able to pivot. Commoners are stewards over their shared-pool resources. They treat these with the same quotient of caring that they bring to one another. Commoners are maintainers as well as innovators. 
Commons can also fail. They can lose the capacity to own and steward the shared pooled resources they need; this will happen should their resources become "enclosed" by another organization (e.g., when these are sold to a for-profit concern). Or, a commons can fail to sustain their governance model and lose the necessary volunteer support and membership. When a commons fails, it is important that pooled resources remain in the civic trust, so that a new commons can be generated as needed to manage these. A single civic trust (Accessed June 12, 2020) can hold the property rights for one or many commons, each of which has access to the shared resource. This also reduces the transactional value of the commons, since the commons owns mainly the right of access (including mixing and mining, etc.), and the trust owns a portfolio of other rights. The trust guards against enclosure of the commons' resources, a major cause of failure.

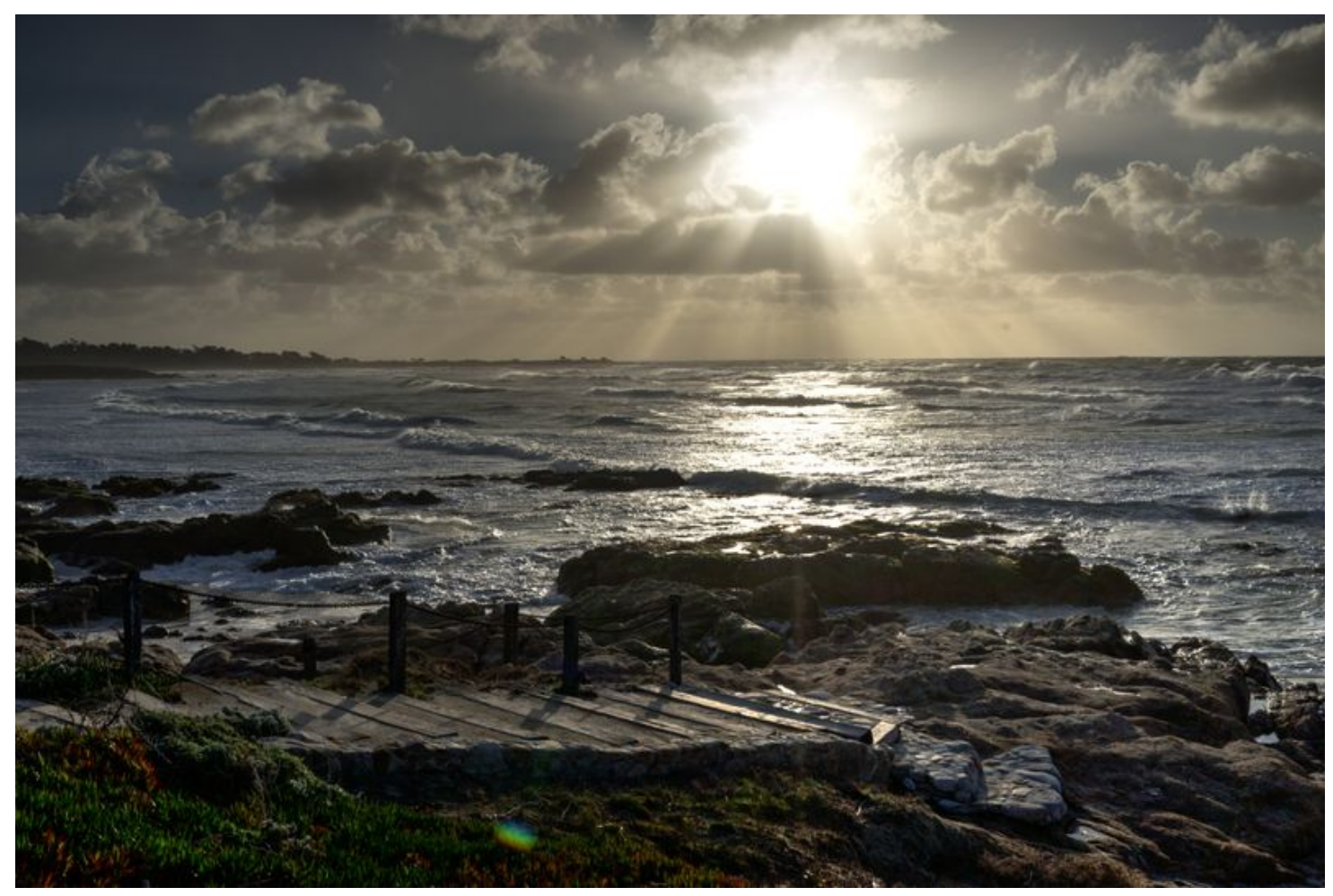

\section{Other ideas/questions about commoning in the academy:}

- A single object-say a dataset-in one open repository can be claimed as a resource by more than one commons, as long as each commons supports their own dark archive, or points to a collective dark archive, in case of data loss. 
- Scholarship needs to be fearless in its infinite play. One role of academic tenure was to protect this condition. In the face of the neoliberal market, tenure has failed in this role. Hundreds of thousands of scholars will never achieve tenure. Can the commons provide this protection?

- Someone noted that many science data objects are "uncommon" objects that require knowledge and knowhow to use and share. Scholarly commons also maintain knowledge and knowhow along with its shared resources. Commoners are maintainers too.

\section{Moving ahead}

The real question is how to rescue (or re-place) current academic institutions using commons-based societies and economics. The commons is not an alt-academy, it needs to refactor existing organizations, where possible, and spin up new ones as required. How can we help move this process forward? If commoning is the "WD40" to release science for the sclerotic hold of its 19th Century institutions (See: Is my learned society obsolete?), internet-based technology is the duct tape needed to help these hundreds and thousands of commons communities work in concert across the globe. The internet holds the future of science. Shared resource platforms, such as Zenodo $<$ https://zenodo.org/> can operate at a global scale when needed, and support thousands of small teams as required. For commoning to gain traction in the academy, we must explore commoner-owned online platform cooperatives (Smichowski 2016) as a generative practice for open science.

The first step is culture change: we need to unleash a more profound understanding of the circumstances of scholarly commoning by building up new open practices, informed by fierce equality and demand sharing. These efforts will be localized and applied as needed to yank local institutions away from hierarchy, intellectual property wrongs, and the pull of the margin that preempts ethical decisions and norms.

\section{A text with some history}

This above text originated from an early-draft document entitled Principles of the Commons, put together by various contributions to Force 11 working groups over the past six years. That draft version of the Principles of the Scholarly Commons was based on the workshop Re-imagining Scholarship held by FORCE11 in Madrid, Feb 2016 and further refinement by the Scholarly Commons Working Group. The original Google Document for this was the product of contributions by several people. You can check in on the current work of this endeavor here: <https://www.force11.org/group/scholarly-commons-working-groupwp2principles/principles-scholarly-commons-open-comments $>$. Hop on and add your ideas. 
The text has been highly revised and edited to introduce the central tenets of academy commons and commoning for this Handbook. PLEASE NOTE: The text no longer expresses the specific recommendations nor the wording of the Force 11 document. 


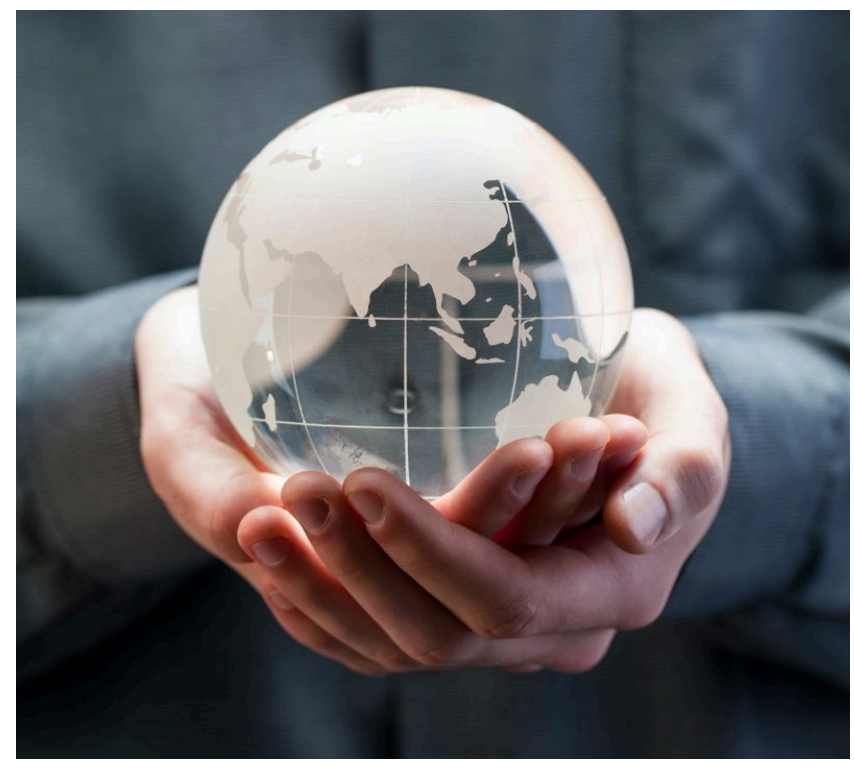

\section{Open Science needs Online Organizations}

\section{First let's look at community}

All of the organizations, the teams, and the networks of science work inside an envelope of community. Community is the container for organizational culture. Commons are governed by and for their community. Learned societies foster community. Agencies fund community (sometimes). So getting a handle on community is a good first step.

The role of community may be the most important, and least understood, aspect of developing and sustaining knowledge-sharing activities. It would not be an understatement to claim that knowledge sharing rests as much on community as it does on technology. To understand why this is so, it is important to understand that community is two things at the same time:

Firstly: community represents a social/cultural container, it describes the cohort, and defines the membership for a group. This is the main way "community" is used and misused.

Note: "community" is not a convenient euphemism for "database."

Secondly: community also describes the qualities of cultural interaction within this group, a shared sense of belonging and trust. The amount of community in a group determines the level at which individuals will voluntarily support the goals of the group. This second sense of the term "community" is what people are talking about when they propose to "build 
community". Building more community into an organization or group gives each member a greater stake in the collective goal.

To makes things clear, let's agree on terminology for the following section. The term "community" will be used to describe the social container and "community-sense" to describe the quality of shared belonging and trust within the group. A community is a group where the members share community-sense. A "weak" community is a community where the community-sense is low and a "strong" community is one where communitysense is high.

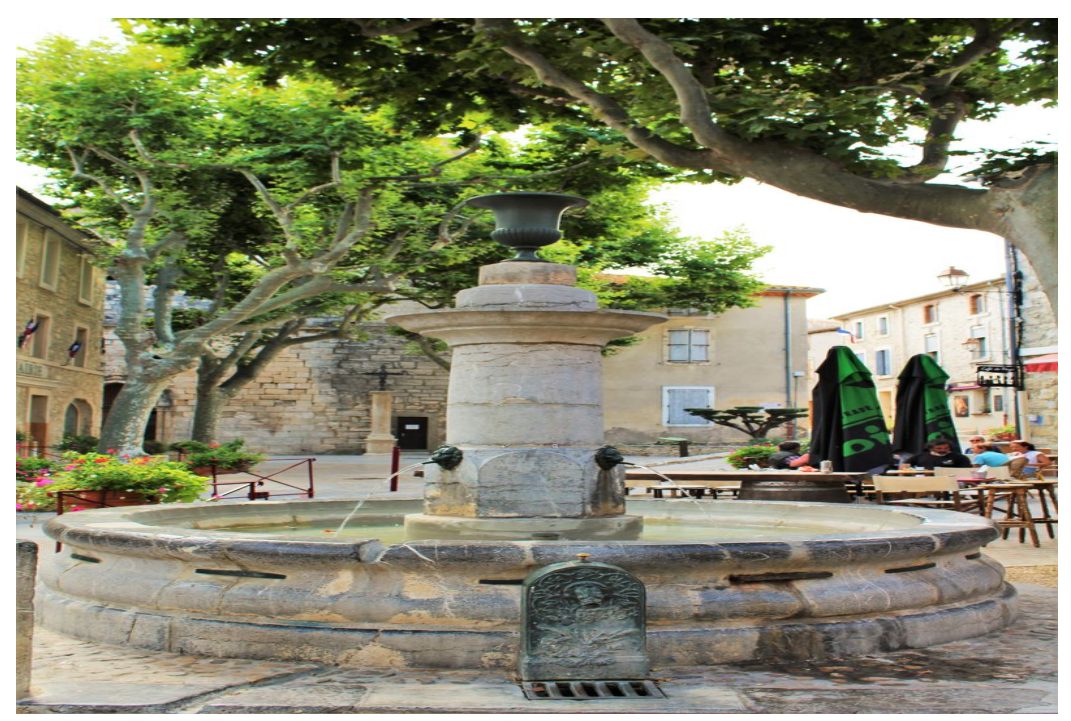

\section{Community Sense}

"Community-sense" is also a term used in social psychology (McMillan and Chavis 1986; Chipeur and Pretty 1999). Community-sense is what Wenger calls the "community element" of a community of practice (Wenger et al. 2002). On the sociology side, community-sense also implies membership and consequent obligations, practical and moral. Community-sense provides the impetus for the informal community sanctions that help prevent "free-riders" from benefiting from the work of the community (Thompson 1993). Community-sense "cascades meaning" (See: teamwork, below) across the community by enabling internal goods: virtues that are expressed through normative practices at various organizational levels (Buckingham and Goodall 2019). Communities are societies that can say "People here do things like this. Come on and join us if you can agree."

Community-sense is the engine for social capital (Putnam 2000), for shared trust (Fukuyama 1995), shared identity (Marcus 1992), shared intimacy (e.g., friendship) 
(Giddens 1991), and reputation (Rheingold 2002). On a grander scale, Anderson (1983) uses an "imagined" community to describe national societies, while the Drucker Foundation (Hesselbein, et al. 1998) posits that community-sense is the answer to many current social problems. Caron (2003) also notes that communities may not be universally positive in their social consequences (remember Jonestown).

There is also a growing literature on community (Koh, et al. 2002, Smith and Kollock 1999), and community-sense (Blanchard and Marcus 2002) for virtual organizations, online networks (Cosley et al. 2005, Butler et al. 2007), and weblogs (Broß, Sack and Meinel 2007). Most of these apply some aspect of knowledge management (Finholt, Sproull and Keisler 2002) or social science (e.g., motivation research (Cosley 2005), emotions (Tanner 2005)).

\section{Teamwork}

Much of the literature on effective teamwork also points to shared values and meaning. Community and community-sense act at different levels at the same time in your organization. Your university campus has (one can hope) intentional community-building efforts to promote shared meanings across all of

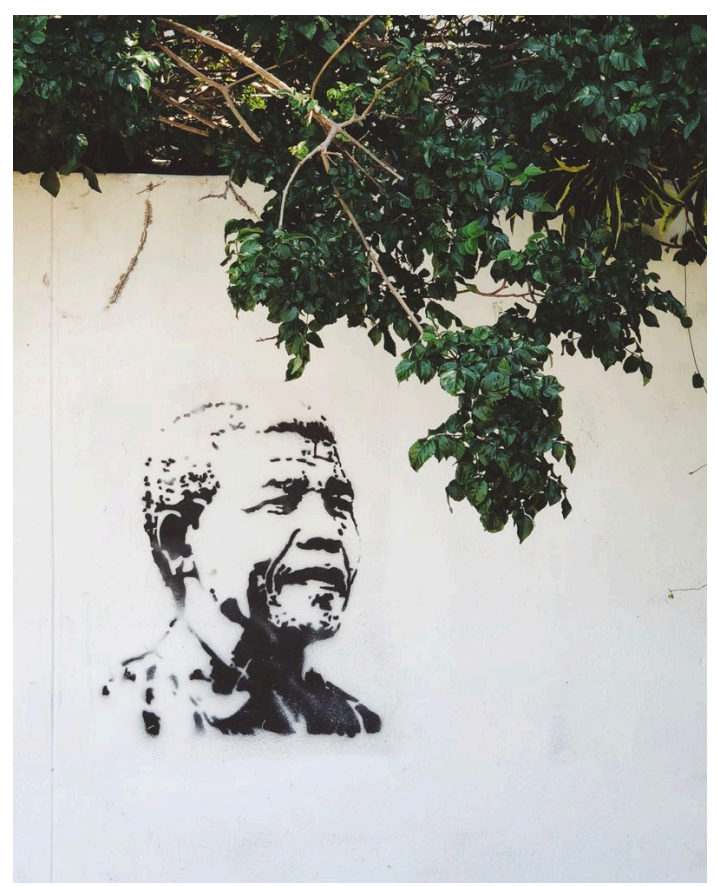
its groups. At this level the meanings/values may be global and broad: "advancing knowledge," "promoting public literacy," "enabling the next generation of scientists." These help to elevate the amount of shared meaning across the organization. You are all in this together. These meanings cascade through the levels of your organization, with each level privileging practices most meaning-full for their work. The main locus of culture and culture sense for you is not the whole organization-although this top layer is important over time-rather it is the group you interact with daily: your team, your lab, your department. Here is where community valorizes (or not) practices of kindness, courage, honesty, and justice (MacIntyre 1984). 
"If one wishes to distinguish leadership from management or administration, one can argue that leadership creates and changes cultures, while management and administration act within a culture" (Schein 2010).

\section{Double-loop Governance is the launchpad for open science collaboration Double-Loop Governance}

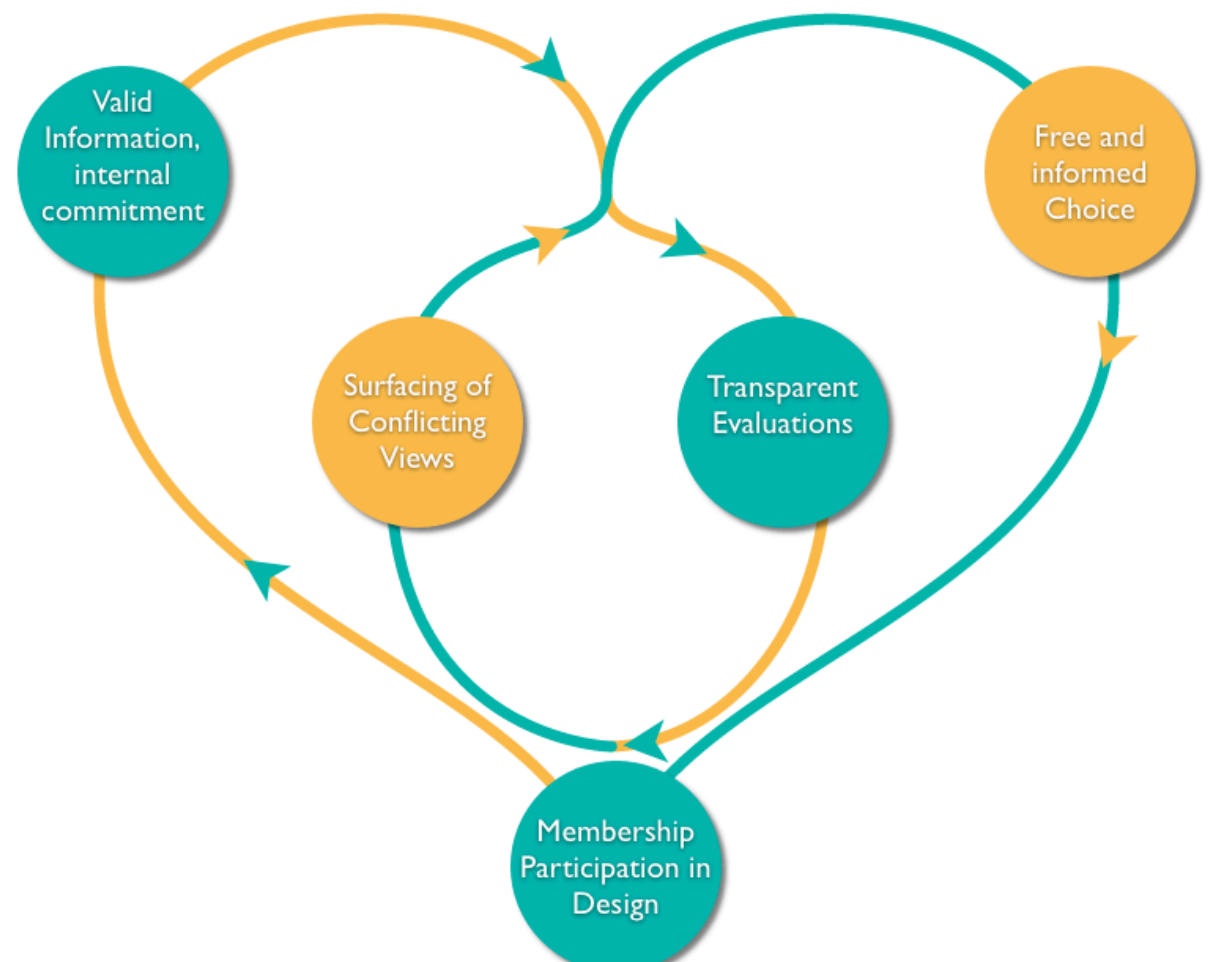

\section{Supports a Liquid Network Environment}

Steven Johnson [Accessed 1/15/2020], uses the metaphor of "liquid" to describe the optimal network environment to enable innovation (Johnson 2011). "Solid" networks are too stiff to pivot toward "the adjacent possible" where new ideas sprout. "Gas" networks are too chaotic. "In a solid, the opposite happens: the patterns have stability, but they are incapable of change. But a liquid network creates a more promising environment for the system to explore the adjacent possible." (Ibid).

More specifically, liquid networks-and the academy organizations that create theseenable individual researchers and teams to explore the adjacent possible; "When the first market towns emerged in Italy, they didn't magically create some higher-level group consciousness. They simply widened the pool of minds that could come up with and share 
good ideas. This is not the wisdom of the crowd, but the wisdom of someone in the crowd. It's not that the network itself is smart; it's that the individuals get smarter because they're connected to the network." (Ibid). The room makes everyone smarter; these new everyones make the room smarter. You need to find/build that room. When you do, you use demand sharing to pull the information and knowledge you need right now to move ahead in your research.

The liquid network is another way of talking about network diversity, the optimal mix of strong ties, weak ties, and strangers in direct communication (See: Ruef 2002) that is a key predictor for innovation in the global elsewhere your research can call home. How do you get this home? The most reliable starting place is to build a culture of organizational learning into your organization. Double-loop governance is a durable platform on which to develop liquid networks across the academy, or in your lab or your department, and at your learned society.

"Double-loop" governance is one example of the type of organizational governance you can establish within the open science culture of academy organizations. Here are some some ideas and some suggestions as to why you might want to consider this form of governance as the heart of your network, laboratory, department, school, college, university, science funding agency, learned society, scholarly commons, etc.

A double-loop governance system brings the values, the vision, and the underlying assumptions of an organization into an open and transparent decision cycle. Double-loop governance is an application of (and a facilitator for) organizational double-loop learning (Argyris and Schön 1978; Argyris 1977; Accessed September 15, 2020). As you will see, learning is not just a public good produced by the academy, but a logic that can create open, innovative governance for the academy.

\section{Four practices at the heart of double-loop governance}

This sensemaking/decision process is characterized by the following:

1. distributed (shared) participation and control;

2. free and informed choices;

3. public testing of evaluations; and,

4. an ability to manage conflict on the surface of discussion threads.

Members in an organization with double-loop governance have the ability to redirect, refocus, and recommit to the values and the vision of their organization. Double-loop governance creates actual peers for a peer-to-peer network. Membership is well-defined, and provided with responsibilities and rewards. 
Double-loop organizations on the open web tend towards do-ocracies and value contributions over any other kind of clout. Decision-making-to the level of deciding underlying assumptions - is distributed rather than top-down. Contributions to decisions and work toward goals (software code contributions, etc.) can be used to measure the value of members, and to reward their service. The metrics for acquiring merit are ideally welldescribed and collectively fashioned.

"These studies [of peer communities building software and platforms] describe governance structures grafted over these systems: usually meritocratic-mostly "do-ocracy" (government by those who show up and do the work); heavily consensus oriented (but requiring only rough consensus rather than creating a veto-rich environment of absolute consensus, and only among those who do the work and show up); a substantial degree of irreverence; redundant pathways so as to avoid conclusive decision making; rare use of formal processes; never of law or managerial fiat" (Benkler 2013).

A great example of this is StackExchange. Clay Shirky (available at http://archive.org/ details/drupalconchi day2 keynote clay shirky), describes how StackExchange uses double-loop governance to engage its members. Double-loop organizations on the web are better able to discover and reward emergent leadership and harvest the long-tail of community participation.

Much of the added value of a double-loop governed organization comes from the quality of interpersonal interactions and shared culture and meaning, the extra amount of available trust, and the additional flexibility that distributed decision making provides. This value does not arrive without additional costs (which are described below). For ventures that are designed to solve a single problem and then end, these costs may not be appropriate. But for academy organizations looking to grow creativity and innovation across decades, the returns on these costs are significant.

\section{A closer look at double-loop governance}

The Handbook didn't invent double-loop governance. So let's look at the history of this notion. It will not take long before you can start to imagine the activities that will drive new governance practices for your organization. A bit of jargon here. Sorry. Double-loop governance puts into practice what Argyris calls a "Model II style" of "theory-in-use". A "theory-in-use" cultural practice describes the actual logic behind actual decisions, as opposed to any announced (but not actually performed) theory. Do not let the terminology trip you up here. Theories in use simply describe the way people really make their decisions, even when they claim to have other reasons and rationales.

\section{Theories in use}


A "Model II style theory-in-use" cultural practice is characterized by valid information, free and informed choice, and internal commitment (Smith 2001). Model II supports doubleloop learning: an ability to question an "organization's underlying norms, policies and objectives." (Argyris and Shön 1978; quoted in Smith 2001). This ability-which all nimble online organizations require to keep up with changing codes and capabilities-needs to be established as a cultural goal of the organization. And for this, it needs to be a visible part of the organization's governance scheme. You'll find out how to do this very soon.

A Model I "theory-in-use" for Argyris (1982) represents a set of strategies-personal and social-that include: control over the actions and emotions of others, maximize winning among peers, and rationality as an alibi for maintaining the status quo. These strategies activate mostly unexamined (and usually unexaminable) assumptions, creating everyday practices while actively resisting reflection. "[I]ndividuals must cover up that they are acting as they are.... In order for a cover-up to work, the cover-up itself must be covered up" (Argyris 2004).

Various experts have described the "covering-up the cover-up" aspect of interpersonal relationships in companies (and universities, etc.). They all point to this as the primary obstacle to any transformative change. Until hidden assumptions (personal and institutional) are revealed and understood, any other changes are temporary and ineffective. Finite-game theorists (such as Carse 1987 and Sinek 2019) describe this situation as a role that the person forgets is only a role; like a stage actor somehow becoming the character they play on the stage. In Leadership and Self-Deception (2010), this hiding is a fundamental betrayal of the humanity of other workers; a betrayal that puts the leader "in a box" where they can only blame others, and treat them as less-than-human. In sociology this situation describes the unreflective "habitus" (Bourdieu 1990) that individuals acquire from their cultural surroundings. The psychologist Carl Rogers (1961) calls this the "social self" that the child builds to defend them against outside threats.

An individual learns Model I "theory-in-use" strategies and covering-up in childhood-it's common wherever parenting techniques teach conflict avoidance- and throughout life they become ever more skilled at these strategies. This is one reason why change (personal or organizational) is much more difficult than simply announcing the desire to change. Individuals are skilled at resisting changes; at an active disregard for what they do not wish to know. As Argyris notes, most people caught in Model I excel in "skilled unawareness and skilled incompetence" (2004).

"Skilled incompetence," Argyris (1986) claims, is why clearly stated plans executed with skill might end up failing to meet their goals. The reason is that the actual, but hidden (with the hiding also hidden) goal has been met: conflict has been avoided, nobody in a position of power got upset, and a whole list of unspoken assumptions remained unnoticed, and their unnoticed condition remained unspoken about. 
The stated goal, perhaps a restructuring of the office work, or a whole new way of communicating decisions, never really had a chance. Model I "theory-in-use" strategies promote and enable only a single loop of learning. Basically your organization continues to get better at incompetence, to excel in unawareness. There is change possible in this loop, as long as this change supports emotional control, opportunities for winning inside the loop, and a default to reasonableness. Over time, change can be visible. Take a look at the Ford Thunderbird image below.

What 24 years of single-loop learning did to the Ford Thunderbird

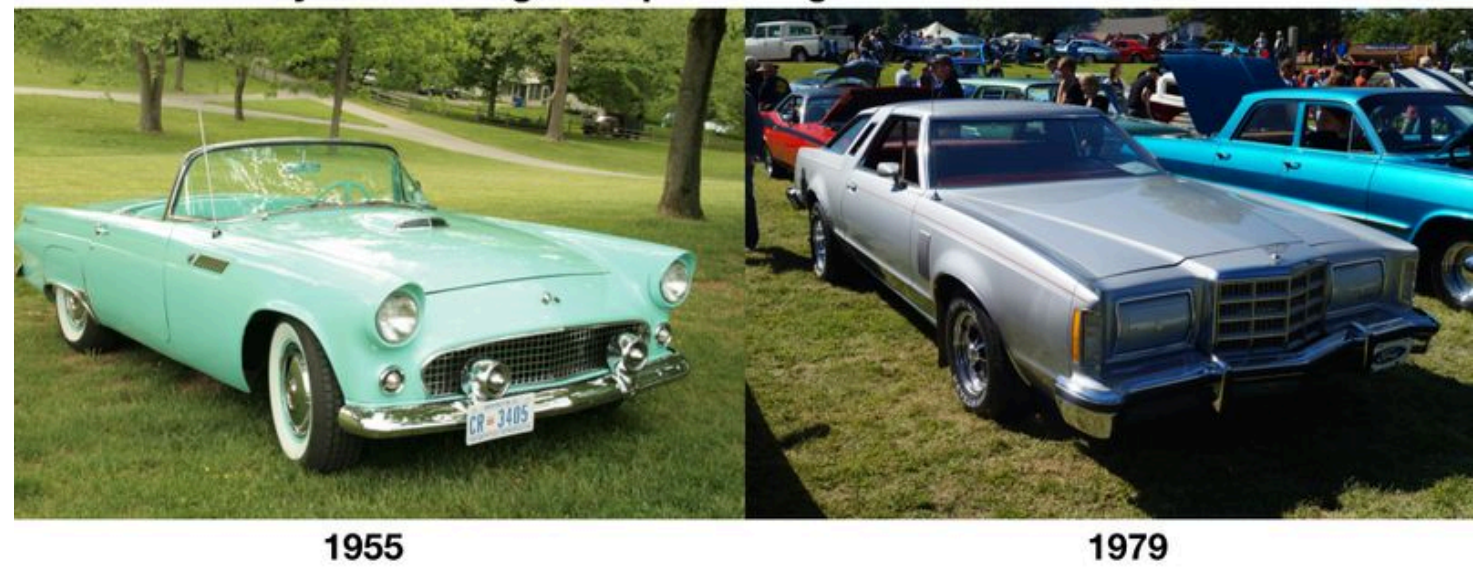

How much skilled incompetence is visible here?

\section{Single Loop Management}

Model I "theory-in-use"-based organizational management provides a single loop of internal communication and learning. Goals, strategies and techniques are attempted and their outcomes evaluated. On the basis of this evaluation, new goals, strategies, and techniques are attempted. All changes happen within the loop. The goal is to improve results using the current methods. This is essential mid-Twentieth Century business management guidance. How business was done.

This is also, in part, why so many Twentieth Century corporations are no longer here. Disruptive innovation and other rapid market changes cannot be addressed through efficiency alone. John Kao (2002) describes it this way, "We all want benchmarks to get the job done more efficiently. But this does not lead to disruptive, game-changing innovation, the stuff of which organizational renewal and competitiveness under conditions of uncertainty are all about."

Government science agencies are also good examples of single-loop governed, problemfocused, service-delivery organizations. They work under externally-mandated goals and 
priorities. Even their single-loop quest for greater efficiency is sometimes constrained by legislative demands and regulatory road-blocks. These constraints provide a motivation for agencies to partner with double-loop online organizations, or double-loop universities. Agencies can borrow disruptive innovation capabilities built into these external groups. The hope, however, for open science, is that these partnership can also seed culture changes inside science agencies.

\section{How to grow a double-loop culture in your university or agency}

Tony Hsieh is famous for saying "your culture is your brand" (2010). Your vision statement, including your core values, is the center of your organization: "We believe that it's really important to come up with core values that you can commit to. And by commit, we mean that you're willing to hire and fire based on them" (Ibid). When "your governance is your culture," the members can more fully commit to the organization. This makes many subsequent (and consequent) tasks that much easier.

Done well, culture is not just an asset, it is an engine for double-loop learning within the organization, and that, in turn, is the engine for shared knowing. Lehr and Rice (2002) make the following observation; "Double-loop learning is where knowledge is generated from information." Done poorly, culture becomes either decorative or punitive (something that employees are required to memorize). Vision and value statements can and should be early Loop 2 outcomes.

Single-loop organizations also have vision statements. What they lack is the built-in capability to question the underlying assumptions of these. Charters, statements of values, and constitutions are all indicators of double-loop governance, although the amount of double-loop capabilities rests in how much reflexive authority they give to members.

\section{Vision and action}

The vision statement, as Sinek reminds (2009) us, is the public statement about why an organization exists. Mission statements/business plans are Loop 1 outcomes. The mission statement tell us how the organization "intends to create [the] future" (Ibid). The "how" is firmly in Loop 1. This is further articulated in business and strategic plans, and then in policies that direct activities.

The "why" lives in Loop 2, and is embodied in the values expressed through the vision statement. The why-the vision, expressed as values-is often described as the "culture" of the organization. Beyond the "why" in the academy, we find a host of "just causes" (Sinek 2019). These are imbedded into the research of every scholar, and into the process of teaching new scholars to learn more about through infinite play. Just causes are the "why" imbedded into the thirst for new knowledge, the promises of new understanding, and the 
joy of discovery. Above we saw that learning is integral to culture-making. There are models for learning that apply directly to intentional learning (and knowing) within organizations.

Schein (2010) puts it this way: "When we examine culture and leadership closely, we see that they are two sides of the same coin; neither can really be understood by itself. On the one hand, cultural norms define how a given nation or organizations will define leadership -who will get promoted, who will get the attention of followers. On the other hand, it can be argued that the only thing of real importance that leaders do is to create and manage culture; that the unique talent of leaders is their ability to understand and work with culture; and that it is an ultimate act of leadership to destroy culture when it is viewed as dysfunctional."

\begin{abstract}
"Human beings learn their theories-in-use early in life, and therefore the actions that they produce are highly skilled. Little conscious attention is paid to producing skilled actions; indeed, conscious attention could inhibit producing them effectively. This leads to unawareness of what we are doing when we act skillfully. The unawareness due to skill and the unawareness caused by our unilaterally controlling theories-in-use produce a deeper unawareness; namely, we become unaware of the programs in our heads that keep us unaware. The results are skilled unawareness and skilled incompetence" (Argyris 2004).
\end{abstract}

\title{
Double-loop governance defeats self-deception and clever leadership
}

The main obstacle to culture change is the built-in, skilled incompetence of leaders on your team, in your department, at your lab, heading up your college, running your agency, or funding your research. It turns out that most of us (this is widespread) have gotten really good at sabotaging efforts to change the culture of our organizations. The bulk of business leadership literature seeks to address this. You don't need to go there.

Brené Brown, speaking on a recent podcast, put it this way: "The biggest barrier to effective teams is not professional development. It is personal development. And to put it in the most bluntest terms I can, people do not take care of their shit. People are not doing their own work on what it is that gets in the way of them fully showing up as the kind of people we need in teams and the kind of leaders we need. It is what makes or breaks a team and it's what makes or breaks culture or leaders is how well do you know yourself, how willing are you to show up vulnerably in relationship with other people; learn, listen, and grow" (Slack Variety Pack Episode 22).

The idea that you can be skilled in incompetence as well as competence has probably occurred to many in the academy who have seen peers become administrators with 
dubious results. But that is not what Argyris is getting at. The anthropology-professorturned-provost with zero management skills is simply incompetent. No skilling implied. However, the dean who can deftly manage a whole room of professors in order to get buy-in on a new endeavor is likely to be extraordinarily skilled at his incompetence. He has just surrendered the opportunity to open up the actual confrontation that might trigger an honest conversation as the start for the process of change. He can never move the group into implementing a new plan with full success. He simply succeeds in avoiding blame and pretending that everyone likes him: it's the same skill he used when he was a school-boy at six years old, and he's only gotten better at it.

What he succeeds in is not upsetting anybody in the process of announcing a program that will fail as certainly as all the others he has offered. The dean's people skills have deflected the real issue: change will be painful. It will challenge individuals to step up and "learn, listen, and grow." Any conversation where nobody gets at all upset is probably a conversation without effect. We will see how double-loop governance uses conflict to achieve transparency and change. You can also check out the section on congruence (Congruence) for more about how a congruent organization can help each member "take care of their shit."

Argyris puts it like this: "The ability to get along with others is always an asset, right? Wrong. By adeptly avoiding conflict with coworkers, some executives eventually wreak organizational havoc. And it's their very adeptness that's the problem. The explanation for this lies in what I call skilled incompetence, whereby managers use practiced routine behavior (skill) to produce what they do not intend (incompetence). We can see this happen when managers talk to each other in ways that are seemingly candid and straightforward. What we don't see so clearly is how managers' skills can become institutionalized and create disastrous side effects in their organizations" (Argyris 1986).

If the worst administrators are just incompetent, and the best ones seem to be skilled at their incompetence, how does anything change? When you boot-strap a double-loop governance to your organization, the practice of doing this creates an engine of change (when needed) and a transparent foundation for stability in the future.

Five ways that double-loop governance can save your organization from itself

\section{Double-loop governance makes every member a caretaker of the vision and values for the online organization.}

Your values are not just a bulleted list on your website nor a poster on the wall. They are the deep logic of why your organization exists. When you create the knowledge loop that includes questioning and reaffirming your values into every decision, then your staff and 
volunteers can celebrate these values. Membership includes embracing the values, and entering into the ongoing conversation about them that keeps them current and vital.

\section{Double-loop governance makes a virtue out of transparent decision making.}

Transparent here means available to all members (not necessarily public). Practically, transparency includes time and place availability. Members are told when and where a decision is being made. For an online organization, this might be a set period of time to edit a certain wiki, or a set period in which to vote online. The management of critical-path decisions may (and usually should) devolve to active subgroups charged with delivering the outcomes. These subgroups need to maintain their own transparent decision process. A great example here is Wikipedia, where each entry contains the edited text, a history of edits, and a discussion page about the text and its edits.

\section{Double-loop governance brings conflict to the surface.}

Conflict avoidance is a major source of "unusual routines" (Rice and Cooper 2010) in general, including those that create institutional guilt. Conflict can arise in many forms. Personal issues surrounding time commitments, responsibility and authority, and expectation management cannot be avoided through double-loop governance alone, but they can be openly addressed and resolved in a manner that promotes reflective learning among those involved. Evaluation conflict avoidance happens when tests of deliverables are either postponed, curtailed, or done in private.

Double-loop governance supports open and thorough testing, and the disclosure of competing interpretations. Conflict is rapidly promoted to the surface of discussions, where voices of dissent become available to all members. Resolution is commonly achieved through a working consensus, not $100 \%$ agreement, but something more robust than a simple majority. Conflicts over the underlying assumptions of the organization can result in new values and a new vision: the organization is free to pivot toward a novel direction at any time.

\section{Double-loop governance accelerates failure to ensure success.}

Remember that double-loop governance supports double-loop learning. Single-loop learning focuses on avoiding failure. Double-loop learning focuses on using failure to recalibrate the underlying assumptions of the activity, this promotes the act of failing as a learning device, and a logic of rapid iterations of activities with open testing. In software development efforts, double-loop governance actively supports agile development decisions. In all endeavors, the ability to fail quickly and recover takes the fear out of trying new strategies. This almost guarantees a better final result. 


\section{Double-loop governance supports do-ocracy and emergent leadership.}

Good organizations find ways to recognize and reward achievements and contributions, better organizations also reach out and cultivate leadership on their edges. One of the benefits of the network effect is an ability to reach out beyond the founding team and find people who have similar interests and valuable skills. As the network expands, the chances of encountering tomorrow's leadership improves. When these people become engaged in activities and outcomes, they need to have a clear path to leading subgroups and then larger groups, and ultimately the organization.

"Some self-veiling is present in all finite games. Players must intentionally forget the inherently voluntary nature of their play, else all competitive effort will desert them.

From the outset of finite play each part or position must be taken up with a certain seriousness; players must see themselves as teacher, as light-heavyweight, as mother. In the proper exercise of such roles we positively believe we are the persons those roles portray. Even more: we make those roles believable to others" (Carse 1987).

\section{Double-loop governance and infinite play}

All of the features of double-loop governance will assist you with infinite play. Distributed (shared) participation and control frees you from choosing a leader and following orders. Free and informed choices come out of real conversations you have, which reach deep into underlying assumptions, where complexity can only be probed, not explained. Your governance can resist rules in favor of shared values and emerging norms. Public testing of evaluations removes the back-channel maneuvers that finite game players use to "win" over others. An ability to manage conflict on the surface of discussion threads opens up the express lanes for continual changes to reflect the emergent nature of doing science as a collaborative enterprise.

Members in an organization with double-loop governance have the ability to redirect, refocus, and recommit to the values and the vision of their organization. Their organization becomes a learning resource for their own personal growth. If this does not resemble your current workplace, then you have good reasons to become an open-science culture change agent. 


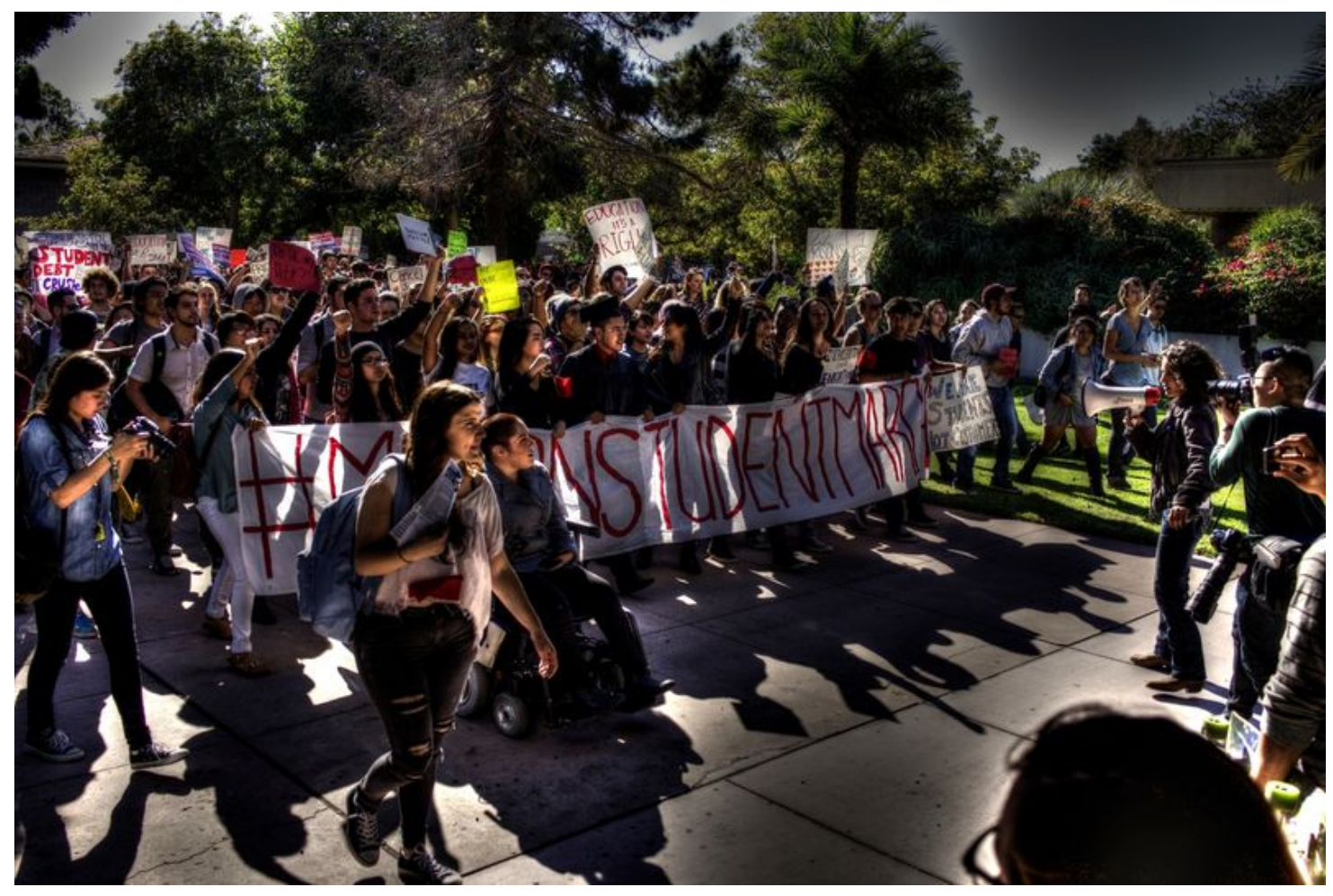

\section{Open Collaboration Networks}

"We need more diverse institutional forms so that researchers can find (or found) the kinds of organizations that best channel their passions into contributions that enrich us all. We need more diverse sources of financing (new foundations, better financed Kickstarters) to connect innovative ideas with the capital needed to see them implemented. Such institutional reforms will make life in the research community much more livable, creative, and dynamic. It would give researchers more options for diverse and varied career trajectories (for-profit or not-forprofit) suited to their interests and contributions"(Kansa 2014; Accessed June 23, 2019).

\section{Build the new, refactor the old}

Looking at the inventory of academy organizations, it seems clear that open science will spawn new research collaboratives in addition to refactoring existing institutional cultures. Libraries will pivot to become central info-hubs for learning across communities of interest and purpose, their collections now digital and their all-digital journal subscriptions tied to actual costs for non-profit open publishing. Ad hoc collaborations will blossom from research conversations on the open web. More focused research discussions will get 
backbone-staff funding to build connected networks around grand (and not-so-grand) challenges. Open, caring programs for learning and social justice with reconnect academy institutions with their locales and regions. All of these collaborations will demand time and resources and will need new career-path support outside of the old dichotomy of "teaching" and "research" (Whitchurch 2013).

The "research" endeavor will expand significantly. Institutional, regional, national, and international repositories will grow to house an abundance of data and information resources offering novel research avenues. Scholarly commons-self-governed collectives formed to optimize shared use of common pool resources, and to secure their maintenance -will range from simple teams inside single institutions, to large, inter-organizational networks that foster a new research enterprise: "Perhaps even more exciting is the prospect that the analysis of vast data collections at scale by machines and through techniques of artificial intelligence will allow us to identify patterns and correlations not visible to human eyes alone. It has been suggested that this heralds a Fourth Paradigm of science. A profound transformation is underway, shifting the capabilities and methods of researchers. This shift is apparent across the research spectrum, from climate science, through genomics, to commercial analytics in the realm of 'Big Data'" (Hodson et al. 2018). Bigger, cheaper, faster: the coming abundance of resources will require new types of collaboration and stewardship.

Celia Whitchurch (2015) notes that these new careers, which often blend professional skills with academic research, will need to be folded into an expanded sense of mission-and new value propositions-for their academic homes. New cadres of academic professionals will be needed to fulfill the mission of open science.

\section{Collectives first, then communities}

In corporate organizational management theories, "community" (such as a "community of practice"), is useful for management as a tool to improve worker engagement, and it also makes workers more willing to share their tacit knowledge, which can then be recorded as institutional memory. "Engagement" in the corporate sense describes a positive emotional alignment of the employee with her work and co-workers.

Engaged workers are said to be more productive (there is evidence for this), and so programs aimed at increasing their numbers have become routine. A somewhat more aggressive form of engagement is called "stakeholder alignment" which looks to build engagement for a specific project. This engagement helps projects move through implementation without hiccups. In the academy, workers at a large public university, say, who are tasked with maintaining the facilities and grounds, feeding the students, providing administrative support, etc., need effective, supportive management the same as workers 
elsewhere. The same is true for the staff of professional scholarly associations and other academy organizations.

Retail merchants commonly do "community engagement." This extends the notion of engagement beyond the workplace to customers, members, or service users, in the drive for brand loyalty (in this case it's also known as "customer relationship management"). At a time when members have simple, powerful means to compare prices and ratings, forging a durable emotional alignment between the merchant and its customers becomes even more valuable. The same is true in the non-profit world where a new army of "community engagement managers" now works to keep donors loyal and their wallets open.

On the upside, the best community engagement programs support an open dialogue to improve the qualities of the workplace, or the product or service. There is a give, and not just a take here. On the down-side, the effort to promote engagement can entail a (seemingly) unending amount of emails or tweets or whatever, designed to remind workers, alums, or customers of why they need to be even more engaged.

What happens when your employees are already engaged in their own research path and teaching arena? This presents a different challenge for universities and academic associations. How do you engage scientists who are already fully engaged with their own research? They don't need the offer of a group tour rate to cruise around New Zealand, nor another term life-insurance policy.

The first rule here is to offer services that help scientists optimize their current engagement without adding more cognitive load (Wilson 2019). Introduce them to the "club" they can join where their pain-points and research friction problems become conversations with peers. These clubs are "...based around the joint production and consumption of scholarly output among a scholarly community. This is an economics of team production and consumption in clubs or, as we style them, 'knowledge clubs'” (Hartley et al 2019). Hartley's clubs center on publication, but really, the next generation of academy organization needs to be club-ified.

"The web, for good or ill, through the way it transforms the economics and geography of both publishing and interactions more generally creates an assumption of access. Perhaps more importantly it restructures the economics of which communities, or clubs, are viable. First, it makes much more niche clubs possible by reducing radically reducing the costs of discovering and interacting with like minded people (those who share cultural elements). But achieving these cost reductions requires some degree of openness. Otherwise there is no discoverability, and no interaction" (Neylon 2015; Accessed July 1, 2020). 


\section{It's a (club) collective, not a yet community: Community can/will happen later}

For several years, studies of online groups revealed a wide range of "stickiness," a description for member engagement. In general, engagement could be plotted on the usual power-law curve; a handful of really engaged members on one side, and hundreds or thousands of mostly un-engaged members in the "long-tail" end of the curve.

One genre of online groups completely broke this curve. These were the most engaged groups online, and by a long ways. Their entire membership regularly contributed content. The problem-for them most of all, and for any online community manager trying to emulate their engagement on the open web-was that these groups were made of individuals who had been diagnosed with terminal or incurable chronic physical diseases.

These online groups, numbering in the hundreds, shared personal stories about symptoms and medication advice, uploaded and argued over new medical findings, and identified sources of emotional support for members and their families. They sought answers beyond the ken of their individual medical advisors, and they collectively shouldered the news when one of their members inevitably passed on.

The feeling of "community" was evident in their mutual concern, but this feeling was not central in these groups. "Belonging" was not the goal; it was their circumstance, their fate, their bad luck. Nobody was trying to get into these groups. Yes, they grew to care for and about one another. But they didn't join for that purpose. Members joined because the circumstances of their lives brought them to this sad place: a space of collective struggle against a common and specific foe: their diagnosis.

\section{Research collectives are valuable and different}

Let's explore the dynamics of these groups. Each online group focuses on a single disease or condition, from ADHD to Zika. Each member shows up already fully engaged in their own private struggle. What they need and find is an online collective, a place to share what would remain private in any other circumstance. A space of mutual learning. Douglas Thomas and John Seely Brown (2011) have described these spaces in their book, A New Culture of Learning. "Collectives are made up of people who generally share values and beliefs about the world and their place in it, who value participation over belonging, and who engage in a set of shared practices. Thus collectives are plural and multiple. They also both form and disappear regularly around different ideas, events, or moments." For more than a decade, the most engaged groups on the internet have been collectives, not communities. Knowledge clubs of the first order.

The global internet has two virtues: it scales pretty well up to billions of users, and it can host a hundred million independent groups. Online "communities" generally (and always when these are commercial in intent) love to grow bigger. Group size is a key metric. 
Belonging builds the brand. No company wants to say, "sorry, we don't need any more customers at this point."

On the very other hand, online collectives only need to grow to the size that optimizes the group's collective intelligence and variety of knowledge. In fact, you know you're in a collective when you try to join and somebody asks you what you bring to the conversation. Collectives have no long tail of non-participants. The collective may be very sensitive to an internal "signal-to-noise" ratio. The quality of participation is a feature.

To use another analogy (getting away from disease for a moment): if you joined a church congregation-note, this is a West-Coast US protestant anecdote-you're a part of that community, even if you only attend twice a year, and toss in a bit of coin now and then. But if you also join the choir, you enter a collective, joined a club. Everyone in the choir is supposed to-you guessed it-sing. If you just stand there with your mouth shut, people will notice. If you don't show up, someone will likely call you and ask where you are and if you are all right. There is no "long-tail" majority of choir members standing up in the choir loft not singing. The choir has zero need for a "choir engagement manager" to encourage choir members to actually sing. Singing is why members join. And if you happen to suck at it, others in the collective might encourage you to leave.

\section{You are afflicted by science}

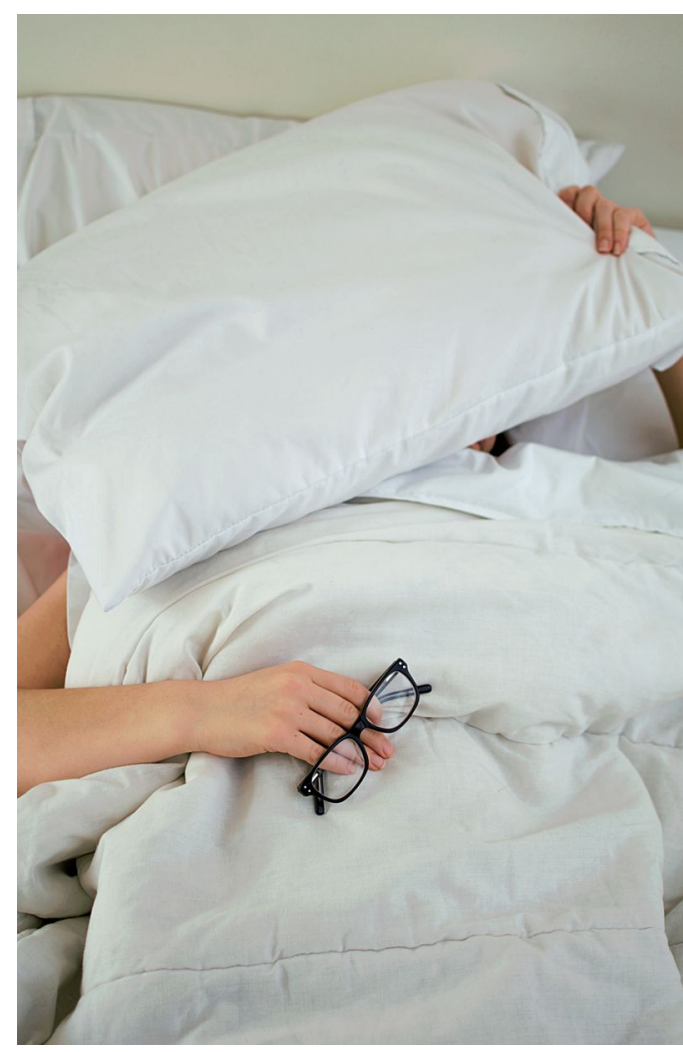

235
From case notes and anecdotes collected from community managers who have attempted to engage scientists online, it is clear that science effects its "victims" (scientists) much like an incurable (intellectual) disease. Scientists commonly spend sixty or more hours a week chasing unknowns in their labs, gathering field data, or tracking down software bugs. They share a fever for knowledge and their own common foe: the specific unknown that stands between the state-of-thescience in their specialty and a better understanding of the object of their study; the peculiar intellectual challenge (disease) they have chosen as their quest and their foe.

Scientists don't need and don't want to join online communities to do science. If that is all your new platform or service provides, your dance floor will 
remain empty. What scientists need are online clubs, collectives that can amplify and accelerate their own research, and reward their contributions to new knowledge in their chosen specialty.

\section{Six lessons so far on online science collaboration:}

1. The most engaged online groups are collectives, not communities.

2. Collectives don't follow the power-law curve.

3. Collectives form around specific issues, and common foes. They house a hunger for collective intelligence in the face of inadequate information. The driver here is a collective need to know.

4. Unlike online communities, membership growth is not a desired metric within collectives. Small can be beautiful.

5. In terms of engagement, science acts like an intellectual disease, a diagnosis of a specific lack of understanding about some object of study that drives the scientist to devote her life to discovery.

6. Scientists will already be engaged if they join an online collective, and will already be disengaged if they are asked to join an online community.

NOTE: community will show up later, because community informs and supports culture.

\section{It's an easy first step: support clusters}

"As they work together in their cells to pursue that goal, the shared commitment of the participants to have increasing impact helps them to build deep, trust-based relationships with each other. This makes it possible for each participant to ask for help from others as they each struggle to get better faster" Hagel (Accessed June 7, 2020).

Your university, online-enabled organization, or newly refactored learned society can start on the right track by allowing members to self-organize online teams, which are sometimes called "cells" or "clusters." Give these teams the teamware they want to use to start conversations across the internet. Help them find others with specific research interests; this is a great use of your database capabilities. Then stand back and let them work. Remind them that clusters need to be active, to get something done early, and then consider unforming. You are not building edifices that must be protected, but rather scaffolds for better conversations that need to be torn down as soon as they might. 
Clusters are a model for the future of online science collectives. They have the virtues of being free, instant, active, and nimble. They can merge with one another or diverge from their original intent as desired. They have no requirements for a deliverable, except that they reward the services of the volunteer time they spend. And so they are motivated to get real work done. They can grow to whatever collective size they need. And when their work is finished they disappear, leaving their findings in a discoverable location on a wiki, a repository, and/or published in science journals.

In a pre-internet world, funding several thousand physical workshops a year helped fill some of the need for science collectives. In the future, internet-enabled science could be based on scientist-led online-organizations that each spawn hundreds of active online collectives as these are needed.

\section{Six more lessons learned}

1. Cluster-like groups can become an important mode of online collective work across the sciences, with huge savings in time, money, and effort.

2. When funders support travel to community-run meetings that grow a culture of sharing and trust, they enable these communities to host their own online collectives. Funders will save hundreds of millions of dollars by NOT directly funding workshops.

3. Each additional cluster can be started with a zero marginal cost (based on existing support for backbone community organizational tools and services).

4. Funders and community staff coordinate among these clusters to amplify the impacts of their results.

5. Funders encourage cross-community online clusters for trans-disciplinary science.

6. Funders can target some travel and other support to improve diversity at the community level. Staff work to nudge diversity at the cluster level.

Platforms and Norms: There's a commons in your science future

\section{Science is broken: Who's got the duct tape and WD40?}

Let's review a bit: Scientists are individually infected with their own science quest. Science is really social too. Why else would scientists take a hundred-thousand airline flights a year to gather in workshops and solve problems together (well, apart from the miles)?

The next bit needs to be about culture and technology. But not so much about the content of culture and the features of technology. Rather, about the doing of culture and the uses of technology. 
Yes, the sciences are broken. Some part of this rupture was built-in (Merton, who outlined scientific norms in the 1940s, also outlined the integral tensions that disrupted these-i.e., the Matthew effect). But much of the damage has come from the displacement of the academy within society that has warped the culture of science.

Yochai Benker generally describes the tensions of this warping as "three dimensions of power". These power dimensions (hierarchy, intellectual property, and the neoliberal need to always show more returns) work against science as a mode of peer production that selfcommits to shared norms. Science needs to find alternative means to fight hierarchy, share its goods, and own its own returns.

The sciences are stuck and fractured, in need of both WD40 and duct tape-culture change and technological support. Scientists need to operationalize open sharing and collective learning. For this, they must discard the institutions that enable the above dimensions of power in favor of new communities and clubs that can house cultures of commoning, and activate global peer production.

\section{The Power of Demand Pulling: a review}

The book, The Power of Pull, (Hagel, et al 2012) announced that the internet had changed the knowledge-world of smart businesses; effectively flipping this from a knowledge pushing activity, where companies used internal knowledge resources to create innovation, to a knowledge pulling activity, where companies harvested knowledge from a broader network of sources and assembled this in novel ways to create innovation. This book was co-written by JS Brown, who had been the head of Xerox PARC, one of the largest knowledge-pushing sites around. This knowledge flip notion seconded what the Cluetrain Manefesto <http://cluetrain.com/> folks had announced in 1999: customers were getting smarter than companies.

In the academy, however, it was business-as-usual, or as usual as might be describable for an early enlightenment project housed in late medieval organizations (universities). In a 2010 blog post for the Society for Scholarly Publishing, Why Hasn't Scientific Publishing Been Disrupted Already?, Michael Clarke wrote, "When Tim Berners-Lee created the Web in 1991, it was with the aim of better facilitating scientific communication and the dissemination of scientific research. Put another way, the Web was designed to disrupt scientific publishing. It was not designed to disrupt bookstores, telecommunications, matchmaking services, newspapers, pornography, stock trading, music distribution, or a great many other industries.... And yet it has [emphasis in the original]."

Clarke's point is that scientific communication should have been the very first arena for radical change fostered by the new opportunities afforded by the Internet; and certainly not the last. His conclusion on why scientific communication had not (in 2010) changed at its 
core-despite the efforts of the open-access publishing (and open-science and open-data) movements-was that publishing had become embedded into career advancement based on the older model (dating back originally to 1680); nobody cared to be the first martyr to a new system, while the old system, however inefficient and expensive it might be, still got one tenure. In short, the reputation of the scholar was carried on the shoulders of the reputations of the journals that publish her works. Journals had become the standardsheraldic gonfalons - that tenure-seeking authors could fly for their deans. But no longer. As these words are being written, open science publishing efforts have pushed back against both the for-profit publishing (more precisely, for-profit "privatizing") model and against the role of journal reputation for advancement.

As an open scientist, you've already moved past relying on seventeenth century publishing logics and onto open access and open review for your work. This means that your research is open to the power of demand sharing/pulling: you have realized the opportunity for others with very similar research interests to discover your work, critique and make suggestions about this, offer questions and new ideas, and push your research into an innovative matrix of collective intelligence. Those colleagues from across the planet who stepped up to respond to your work can become an ad hoc cell shaping the contours of learning toward the goals of exploiting serendipity and increasing research impact.

These small collectives are exactly what your research needs to accelerate into new discoveries; realizing the power of pull, as you activate demand sharing. At any one time you will probably find yourself a member of several internet-enabled cells, each probably numbering fewer than twelve members, and each working to solve some immediate problem. Once their problem is solved, the cell can publish the solution and simply disband. All that remains are the affective ties of trust and gratitude that its members retain, each one building their own network of collegial acquaintances, "weak ties" that will grow and mature over time.

Amassing a social network of "weak ties" is one of the most important and valuable practices that any scientist can do, not just for science, but for their own careers. "If information is to move from one group to another that is far away, either socially or physically, then the only way is through bridges-links between two different people, two different worlds, links that, by definition, are weak rather than strong. As Granovetter (has 1977) wrote in his original paper, 'this means that whatever is to be diffused can reach a larger number of people, and traverse greater social distance, when passed through weak ties rather than strong'. If weak links are removed — the bridges, as it were, are blown upthen this would harm the spread of information more than if strong links were dissolved. If the bridges did not exist, new ideas would be stunted or spread slowly, science would be handicapped, and social divisions would be perpetuated" (Koch and Lockwood 2011). 
Let's turn now to these new "weak link" open collaboration networks, and to internal reorganizations that academy institutions face as they grow into open science hubs. 


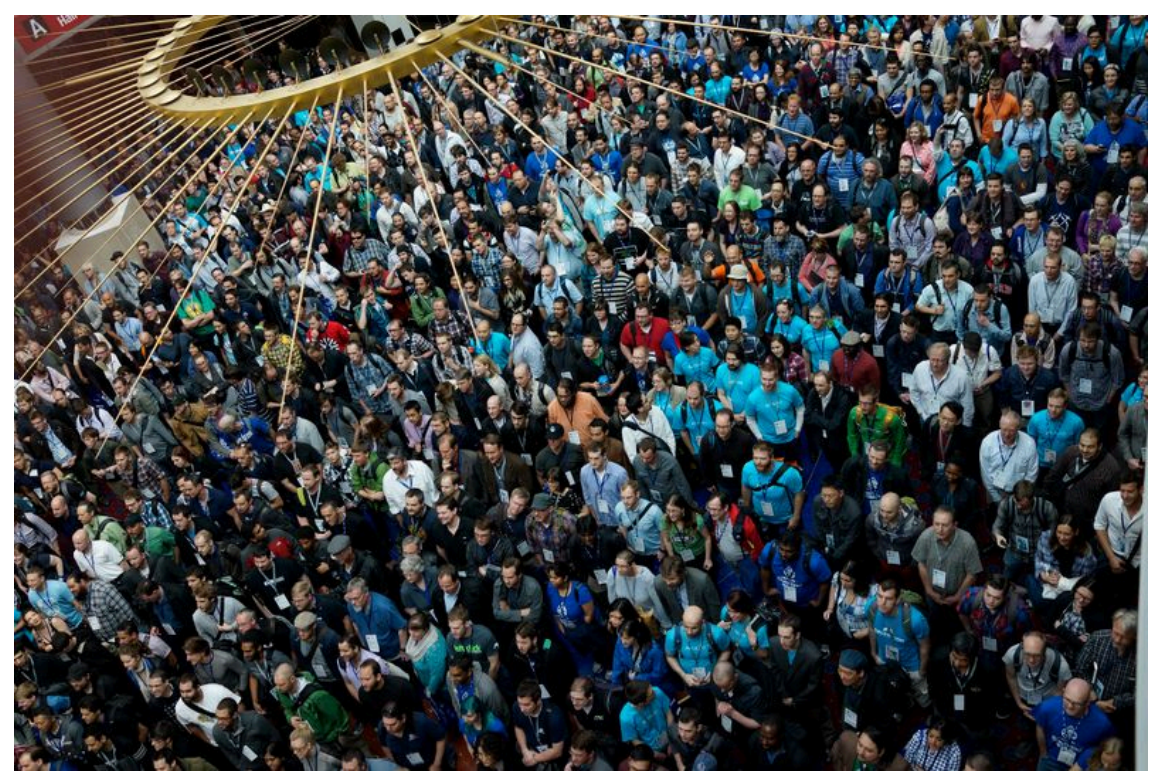

"[N]ow that science is becoming a network, knowledge is not something that gets pumped out of the system as its product. The hyperlinking of science not only links knowledge back to its sources. It also links knowledge into the human contexts and processes that produced it and that use it, debate it, and make sense of it. The final product of networked science is not knowledge embodied in selfstanding publications. Indeed, the final product of science is now neither final nor a product. It is the network itself-the seamless connection of scientists, data, methodologies, hypotheses, theories, facts, speculations, instruments, readings, ambitions, controversies, schools of thought, textbooks, faculties, collaborations, and disagreements that used to struggle to print a relative handful of articles in a relative handful of journals" (Weinberger 2011).

\section{Open Collaboration Networks are the future of science}

One thing that is new and not at all new about open science is the reliance on "online organizations." Learned societies bring together widely dispersed scholars, and have been doing so since the $17^{\text {th }}$ Century, when the advent of postal systems fostered widespread mail correspondence across the universities of Europe. Internet-enabled science organizations are, by definition, no older than 30 years.

Platform coops and gig economy firms are sometimes called "virtual organizations:" companies expanding their workforce without adding more employees, through outsourcing. As the current pandemic (this is 2020) has taught us, any organization can become "virtual" today, with all of its members separated in space and communicating 
across the web. So the Handbook will use "open collaboration networks" (OCN) to corral the capacity to include geographically distant colleagues into a working collaboratory that can harness collective intelligence. As we learned in Science happens elsewhere, no single college, university, laboratory, or agency has the means to assemble all the scientists doing work in even the tiniest sub-discipline. The dozens of scholarly commons that will emerge in the coming decades will all use OCNs to guide their work.

What can you do when your research team looks around and realizes, "This problem is way too complex?" The first impulse-over the past half century or so-would be to apply to a funding agency to run a workshop. Large agencies spend billions of dollars on these; they create an ocean of white papers that lurk, mainly unread, on institutional websites. Elsewhere we discuss how most-but certainly not all-workshops are obsolete today. There are nimbler methods to get the same outcome. Let's explore.

\section{Ashby's Law}

When your government agency or university laboratory looks to innovate in a world where multiple/large data inputs are coming on line, how can you stay ahead of the inherent complexity of the systems you are creating/interrogating? One way to look at this problem is through Ashby's principle/law of requisite variety. A principle of cybernetic management, requisite variety notes that unless the control system has at least the variety of the environment it controls, it will fail. Which actually means that some part of the environment will be controlled elsewhere.

Elsewhere is also where innovation happens; because unless you can corral the inherent variety of the problem you face, it will seem too complex for your team to innovate a response. You can either go out and hire a bigger team, or you can borrow enough requisite variety just long enough to bring your own team up to speed. This is one form of an open collaboration network.

The network you build to tackle a tough problem needs to have enough of a portfolio of knowledge and skills to address all parts of the problem. Not only that, but they need to communicate their skills and knowledge to one another so that each team member shares in this collective intelligence. Andrew Van de Ven put it this way, "Requisite variety is more nearly achieved by making environmental scanning a responsibility of all unit members, and by recruiting personnel within the innovation unit who understand and have access to each of the key environmental stakeholder groups or issues that affect the innovation's development." (Van de Ven 1986).

Open collaboration networks include online communities of practice, research collaboratories, open source software programmer collectives, and other groups in a great variety of arenas and professions. What they offer is an open network of common interest 
and complementary talents. When your university, foundation, or agency is looking to innovate in a world where data are more plentiful than insights (Abbott 2009) then it makes great sense, in terms of time and effort, to fund and build an OCN and gain enough requisite variety to conquer complexity and kickstart some innovation.

So, you want to, or need to start a new open collaboration network, or build one inside the mission of your department, or laboratory. Perhaps you have been awarded some funds to do so. If so, congratulations. If you do this right, it will govern itself into a productive, happy (smiles are a good thing) academic community.

\section{Here are Seven Key Suggestions}

1: Read Jono Bacon's 2009 The Art of Community. Bacon has more good advice than you will find in a hundred blogs. Governance is not the same thing as management: "Don't fall into the trap of assuming that governance is merely about decision-making. There is no reason why you can't constrict it in this way, but you will be missing out on a wealth of opportunities to excite and energize your community." What Bacon will also tell you, and it's very important, is that you need to build your community and its governance first thing. This is not a "phase 2" activity in any plan.

2: Connect with the community on the issue of membership. Who gets it, what levels there are, who gets to vote, who gets to lead, and how to manage conflicts: getting some early conversations done with the community, and particularly those who will be asked to volunteer, will help to draft that part of the initial governing documents. Remember that you are setting up the initial conditions for your member-led organization. Double-loop governance means that your members will be able to rethink membership rules and roles.

3: No matter how much you want to implement a plan with your team, and no matter how you have researched effective governance, you will only be creating a temporary framework for your membership to use as a first go-around for a governance system. Because you are giving your members the ability to make changes in the documents you have drafted, you have to understand this: they will make changes, probably right away before even an initial vote is taken. And then remember: this is a good thing. So, put the texts up on a wiki and let them have a go at it. The sooner they come to own the text, the sooner they will start to celebrate its vision.

4: Put some budget into play if you have this, but never pay volunteers for their time (See below about staff and volunteers and who does what). What can you pay for? Help support communication platforms, pay for students to do some background research for a draft business plan (the "how" of your organization), bring in some key community members for a workshop, but open this up through video conferencing, and included others who express and interest to also be present. 
5: Always work toward a rough consensus, and never erase "minority reports." Let conflicts rise to the surface and deal with them quickly. Leave their content open for others to see. Show your members that their time, their skills, and their opinions are honored, even if they are overruled. Jono Bacon has great advice for conflict resolution.

6: Ignite some preliminary teamwork by having the initial community vote on two or three small, "low hanging fruit" efforts and then support ad hoc teams (clusters) to address these. By this you begin to show an initial innovation ROI the online organization will build upon.

7: Hold a few face-to-face meetings, but keep them from being PPT centric. Plan for smallgroup discussions and multiple breakouts, and hold the meetings in convivial neighborhoods, not airport hotels. Gather as many members as are there and read over the founding governance documents paragraph by paragraph (but only once, and then set up a process to edit the text online until the document goes up for a final vote), and let the group speak their concerns. Open up the entire budget for the membership to give their suggestions. If possible, let the membership vote on the budget after suggestions have been taken and changes made (a real vote).

These suggestions are just a starting point for boot-strapping a double-loop governed virtual organization. Once the hard work of building in double-loop governance into the culture of the organization is over, the rewarding work of seeing how this accelerates volunteer engagement can begin, and the creative work of husbanding this engagement into your organization's business and strategic goals can be fully supported through the culture and the values, and the celebrated vision you own as a community.

\section{Walking the walk is only hard when you haven't tried it}

For many commercial organizations, the rush to market and the lure of some short-term exit strategy might make all this focus on congruence and culture and values and vision seem superfluous. And if your goal is to start-up and sell your business in the next 24 months, you would be wise to stick to a single-loop management plan (with a hefty stock option, because you will not have much love or glory). Similarly, when you have funding for three years, building a governance structure that takes at least three years to find its feet might seem unattractive. The Primary Investigator in you-and certainly the Program Manager you send reports to-wants/needs to show progress in months, not years. So you punt on the governance and push for "results." This is the pathway to innumerable white papers that nobody ever reads.

The main reason why so very many funded-project-led open collaborative networks fail as soon as the funding is over is this: their PI and the agency/foundation PM failed to enable the network community to build their own collaboration infrastructures inside a self- 
governing process. They have no self-governed collaborative capacity to move ahead when funding stops.

If you are tasked to build an online organization that can stand on its community-based resources, you should seriously consider building in double-loop governance from day one. What you are offering your membership (or your employees, or your customers) is a congruent experience: whatever your brand (or your vision) will become, it will emerge directly from your culture.

\section{Your OCN deserves all the Democracy it can Handle}

"What motivates social behavior is a sense of ownership, to be able to say that the commons or a shared good is 'mine' or 'ours.' The more intensely ownership is experienced, the greater the sacrifices people are willing to make" (Klamer 2017).

As an open scientist you may be been tasked-perhaps through a grant, or by occupying a management position in your university_to build or lead an open collaboration network (OCN). Up front, you will face questions about the cost/benefit issues of democratic governance, as opposed to central management. If you stepped into an existing supervisory role, this question is yours to bring to the table as you get a handle on the exiting culture and everyday practices, and begin the process of making these practices more reflexive and transparent.

Given the usual shortage of funding and time, you will have real concerns about the effort required to build a community-based governance system. These concerns are usually layered on top of the more general concern that the community (or rather, certain activists within the community) may use the governance system to push the organization's goals toward their own interests. Managers who desire to manage have little good to say about adding democracy to their process.

\section{Ownership is the cornerstone of community}

While demand-sharing cultural norms call for removing the barriers of private ownership of the academy's scientific intellectual property in favor of commons-based resource governance, organizational governance is based member-ownership of the budgets, processes, and decisions made within the organization. Members own their organizations, and the organizations share their assets among members, and with other academy organizations.

\section{But democracy is expensive in time and resources...}

Certainly, democratic governance increases the overhead (in terms of time and effort) spent on governance. Top-down decision making can be quite efficient up to the point where it 
tends to fail rather abruptly. Democratic governance is also more prone to being gamed by people with time and interest to do so. This is where the community comes in to play. When you build in enough democracy to give the community the opportunity to really govern, it will tend to resist the efforts of certain individuals to subvert this opportunity. This is one of the goods that democracy delivers to your OCN.

What are other goods that democracy provides for your OCN? What does democracy do that you cannot do without committing to this type of governance? There are two types of goods (positive, valuable results) that democracy creates within an OCN. The first type are community building goods. Democracy is central to your ability to build a community for your OCN. The second type are decision support goods. These help align your decision making effort with the goals and vision of the community, and improve buy-in by the community.

The main community-building good you achieve by promoting democratic governance is inclusiveness. The divide between the funded OCN team and its larger collection of stakeholders disappears. Governance provides the means for a wide variety of voices to be recognized. This encourages more participation (and more participants) building the size and the depth of community for your OCN.

The next community-building good is that of bottom-up control. Bottom-up control over your OCN establishes the means for the community to have a say not only as an afterthought (e.g., a survey), but on matters as central as budgets and goals. The community is given real ownership over the OCN's efforts. This is the hallmark of any authentically "community-based" or "community-led" organization. Until your OCN has achieved this level of community governance, your efforts to build a community will be met by a wall of indifference. Developing the means for community-based control of your OCN breaks down this wall and builds the foundation for real community growth.

Decision support goods can also be expected from your democratic governance efforts. Once the community is fully involved, you receive the benefits of their considered judgements. The whole point of your OCN is to engage with larger numbers of people with expertise. Why not put this expertise to good use?

Give intelligent people-your colleagues-the reason and means to reach a considered judgement on an issue of importance to your OCN and they will work diligently in this effort. The second decision support good your OCN gets is new leadership. Those in the community who have expertise, time, and interest will step up to take positions of authority/responsibility for the work that the community is contributing to the goals of the OCN. Find the means to reward these leaders (give them resources to manage and build their reputations within the community) and they will become the levers to take your OCN to the next level. 
Of course, not all democratic governance efforts are equal. Your OCN will need to work to maintain transparency in the governance activities, in part by making the staff fully accountable to the community. Your OCN's governance system will need mechanisms to be modified so that it can learn to be more efficient over time.

Using democratic governance to build community is probably the best solution for crafting a virtual organization that can survive its initial funding. There are no half-way democratic forms that will deliver the same goods. And if you decide to forego democracy, or delay democracy while you build your technology, there will likely be a day when the funds run out and you will turn to the "community" and ask them for their support. Good luck with that.

\section{Why do democratic governance when you are already paying staff?}

Getting the right mix of staff and members for an OCN is a crucial task for sustainability. The key is to take limited resources (if you have unlimited resources, call me) and invest these in directions that bring the best return for all. Member volunteers are the heart of an OCN . Keeping this vital organ alive and well is job one for staff. Members bring skills, vision, energy, and passion to the organization. They tend to do so in short-term increments. They need to know their efforts are valuable. This knowledge prompts them to stay engaged. Through the serial engagement of many members, certain activities are maintained: governance, oversight, incremental work on infrastructure, a supply of new ideas, and, yes, occasional sidetracks. Nobody can sell your OCN to funders and new partners better than members. And nobody can grow your organization over time and on budget like members.

What is your staff good for? Staff are the backbone of any OCN. They keep it on track and guide its fortunes. They have responsibility for those tasks that members should not be asked to perform (more about this soon). They also have responsibility to keep members engaged. They do the thank-less work and get paid for this. But that doesn't mean the organization doesn't owe them a heap of thanks. Still, they are professionals, and need to step up an take charge when the need arises.

Generically, the work of staff falls into two buckets: everyday necessary tasks and putting out fires. members should not be asked to perform these types of work. Staff run the events on the community's calendar; they manage the web-presence, the accounting, the teleconferences, and a hundred day-to-day activities. They facilitate volunteer efforts. And, when the website is hacked, or the projector bulb burns out, they fix it.

Members get called in to plan and direct new activities and articulate new goals. Ideally, they are given a say (not just a voice) in the OCN's operational budget. Because they do the planning and determine the budget, it's only fair that they do some of the work. They can be 
tasked to scope out any new work required by a new goal and to build new capabilities to meet this. Then they either do the work, or determine that the job is too big for them to accomplish.

When the members are done with their efforts, the outcomes are passed back to staff to incorporate into the organization's operational inventory. Sometimes the outcomes are not fully ready to use (having been built by members). Staff might need to hire an outside expert to polish the work. Note: this person should be fully "outside" and not a community member. Never hire a community member as a consultant to fix another community member's volunteered contribution.

When the job is too big, members might ask for some support (more often they just stop answering emails). There are many ways to support members. Paying them is the least valuable, as this transforms them into non-members. There are several descriptions of the negative impacts of paying members. Basically you are pissing in your own soup. Other means of support are always better: find them assistants (pay for interns), pay their travel, pay for hardware and software when required, and, if nothing else works, add staff to help. Sometimes, this might mean making a skilled volunteer a "fellow" for a short period of time. This move should include a community vote, including an open call for the fellow position within the community. The community is tasked to help staff fill a temporary (less than a year) need from within their ranks. By this, the "fellow" can be paid for a time and then return to the ranks of the volunteer community.

Remember that members need to know their efforts are valuable. The organization needs to build and maintain recognition systems for members. These include online and inperson awards. The three motivations for engagement are money, love, and glory. When it comes to members, if you are stingy with the glory, don't expect any love. And when you let the community add to their own glory, then you can stand back and watch new leaders emerge and know your open collaborative network is healthy and growing.

\section{Self-Governed OCNs are a Win-Win-Win for funders too: let them in on this important fact.}

Science agencies and science-focused foundations fund science. It's their job. Usually this is done directly through the competitive funding of research projects, equipment, and centers. All of these competitions can include instructions for the proposers to describe how their work supports open science. You can make it clear in the request for proposals that the outcomes of the project, including software, data, and results, are openly shared, reproducible, and reusable. You can do much more than that, for a very small investmentsay, one percent of your research budget. With that investment, you can help shape the culture changes needed to move your funded research into an open science future. 


\section{Funding open networks}

As an open scientist in a funding agency (government or foundation) or a scientist proposing a new research network, there is something to always remember: some important science-related activities that cannot, indeed must not be directly funded in order for them to succeed. Sometimes innovation needs to go where cash doesn't count.

If you are guiding a science funding agency or foundation, then the notion that you can achieve certain high-value science goals only by not directly funding them may be news to you. It should be welcome news. In fact there are enormous innovation potentials you can only realize when you can refrain from adding money to the mix. There is a caveat here. While you cannot fund these activities and processes, you also cannot manage them. Instead, they must govern themselves.

As the abundance of openly shared research objects grows, self-governed commons need to step up and steward these resources for an optimal return on investment. The time is ripe for new varieties of science/data virtual organizations/networks. Drawing their members from a broad swath of experts, led by the community they build themselves (through a governance they own), and powered by volunteers, these commons offer funding agencies and the academy new forums for scientific discussion, knowledge management, and collective intelligence.

To be really clear; These organizations still need support. All of them require sponsors to pay their staff and expenses. They manage websites, teleconferences, shared research capabilities, and stage face-to-face meetings to build community sense, and expand the "room" of their conversations. But these activities: the occasions for trust building, the growing sense of community, and the actual work: these are ultimately accomplished by the volunteers for themselves and without being paid (apart from some logistic support).

Distributing activities across an engaged volunteer network of peers can use a very limited budget-say five percent of the total research budget-to accomplish the socio-cultural side of the program, given time and proper attention to governance. Money is not the driving force here; trading money for time is not an option. Agencies that fund science research can easily afford to support governance through project research budgets; more to the point, they cannot afford to not support this if they want to accomplish what only a community can do.

At the same time, efforts to support "community" are often pursued without actually building community-based governance. Project budgets may include large amounts of "participant support" for annual "community meetings" that could help build engagement. But without the governance structure that puts the community in a position to determine key aspects of the core activities, these meetings are really only very expensive alternatives 
to an email list. The attendees may learn something, and will forge their own interpersonal connections, but the work of building community through governance is left undone.

The amount of real governance required to support activities also depends on what types of activities need to be supported. If communication is the goal, then a relatively weak governance system will suffice. If real collaborations are to be supported through an open collaboration network, then a robust governance system may be needed. Clay Shirky (2008) examines three levels of social interaction: communication, coordination, and collaboration. Each of these levels needs its own level of governance to be sustained across years.

Funders gain value for their research outcome when they invest in community. But it's the members, the scientists and their teams in these collaborative networks that realize a massive return by their involvement. In fact, each and every scientist should get more than they give. This math is driven by a host of network effects. You, the agency manager, can finally get something for almost nothing; and that something is a thing that no amount of additional budget would achieve!

\section{Seven science outcomes that your agency can only fund by funding self-governance}

Here are Seven Outcomes your science agency can now get only by not funding them directly. Rather, you can achieve these through supporting a community-led virtual organization of scientists/technologists:

1. Your agency gets to query and mine a durable, expandable level of collective intelligence;

2. Your agency can depend on an increased level of adoption to standards and shared practices;

3. It will gain an ability to use the community network to create new teams capable of tackling important issues (also=better proposals);

4. Your agency can use the community to evaluate high-level decisions before these are implemented (=higher quality feedback than simple RFIs);

5. Social media becomes even more social inside the community, with lateral linkages across the entire internet. This can amplify your agency's social media impact;

6. Your diverse stakeholders will be able to self-manage a broad array of goals and strategies tuned to a central vision and mission; and,

7. You will be able to identify emergent leadership and potential new employees. 
Bottom Line: Sponsoring a community-led, volunteer-run science organization offers a great ROI. There are whole arenas of valuable work to be done, but only if nobody funds this directly.

\section{ESIP: a real-life example of a wildly successful OCN}

The oldest and perhaps the best example of a self-governing, online science OCN in the US is Earth Science Information Partners (ESIP), sponsored by NASA, NOAA, and the USGS (esipfed.org). Why is ESIP a good example of these new science organizations? A good deal of the buzz at any ESIP meeting meeting is generated by the appreciation for the "ESIP way" of getting things done. ESIP champions open science at all levels, and this openness extends to everything ESIP does internally. ESIP builds and celebrates a strong culture for the pursuit of open science in the geosciences, and remains a model for other volunteer-run open collaborative networks (OCN) across science domains. There are lessons learned here that can be applied to any arena of science.

\section{Governance NOT Management}

One important lesson learned at ESIP is this: governance must never be reduced to management. Funded projects and centers are managed. OCNs are self-governed. Volunteer-run science organizations are intrinsically un-manageable as a whole (for reasons we shall see soon), and at their best: it's a feature, however inconvenient for agency program managers. An OCN can certainly house dozens or hundreds of small, self-directed teams where real work can be "managed": teamwork is central to their mission. ESIP "clusters" are good example. These teams can produce valuable and timely deliverables for science and for the sponsors.

The style of governance is also very important here. Attempts to shift governance away from the membership and into top-down executive- or oversight committees are always counterproductive. They give the membership a clear alibi to not care about the organization. Academics have enough alibis to not volunteer without adding this one. The members need to own the mission, vision, and strategies. Successful activities will emerge from initiatives that have been started independently, and with some immediate urgency, by small groups; and which grow into major efforts with broadly valued deliverables. Bottom-up governance will outperform top-down management over the long term.

\section{Science culture shifting}

Probably the largest recognized impact that science OCNs can make here-and perhaps only these can accomplish this-is to model a new, intentional cultural mode of producing science. This new cultural model will be centered on sharing (sharing is also one of the oldest cultural traits of science, only recently neglected). Sharing ideas. Sharing software, 
tools, techniques, data, metadata, workflows, algorithms, methodologies, null data, and then sharing results. Reuse needs to become a key metric of science knowledge (Cameron Neylon noted this at the original Beyond the PDF conference).

Transforming science means changing the culture of science. Science OCNs must perform a lot of culture-building work here. This is often a challenge for their sponsors. The key learning moments and opportunities, and perhaps the highest ROI for sponsoring a science OCN is when this community teaches its sponsor to also change their organizational culture.

\section{Three critical governance conditions any agency/foundation sponsor needs to heed}

There are three necessary conditions for an agency-sponsored, community-led organization.

1. The sponsoring agency needs to allow the community to build its own governance. Governance documents and practices are not subject to approval or even review by the sponsoring agency, apart from needing to follow standard fiduciary rules. The sponsoring agency can offer input the same way other individuals and groups do, but the community decides its own practices. The metrics for the governance are the growth of volunteer participation, and spread of community involvement, the perceived transparency and fairness of decisions, and the community's estimation for the value of the work being done.

2. The sponsoring agency has no right to review or in any way interfere with elections. All organization members have the right to run for office and to be elected. If the governance process allows, volunteer leaders might be selected through a lottery, which helps bring in leaders from the margins.

3. The agency's sponsorship is designed to help the organization grow into its potential as a volunteer-run, community-led scientific organization. The returns on investment for the agency are multiple, but do not include tasking the organization to perform specific duties, other than to improve their governance (and their member satisfaction) over time.

As an open scientist, and an open-science culture-change agent, you will want to be able to demonstrate that a small amount of your science funding budget is best spent on building self-governing organizations, instead of direct investments in funded projects. The real test for a science OCN is to develop fully within the scope and logic of its organizational type. The concomitant test for the sponsors is to understand that sponsoring a new and different type of organization will require some new expectations and an incubation period (a few years) of growth and experimentation to allow the organization to find its own strength and limits. 


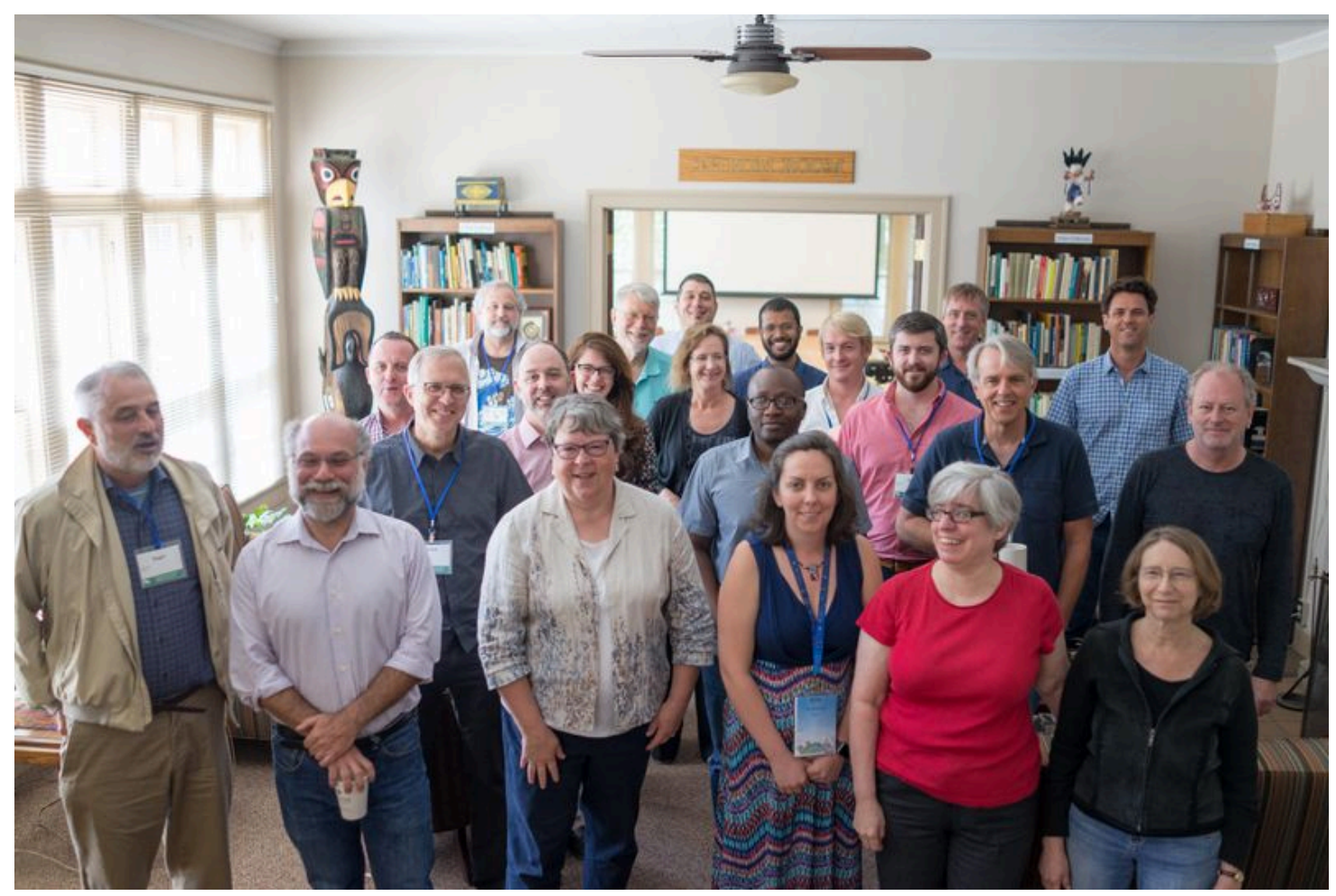

Commoners workshopping

\section{Workshops and beyond}

Workshops demonstrate how much science needs shared knowing.

There are lots of reasons to not run workshops, and a few reasons why these can be uniquely instrumental. The best workshops represent a mode of in-person shared knowing sometimes known as BORPSAT: A Bunch of the Right People Sitting at the Table.

If you can...:

- Know who the right people are;

- Know how to contact them and get them on a plane on the same weekend;

- Know how to facilitate the conversations necessary to draw out their variety of knowing (Knowing and conversation);

- Know how to record this and where to share it; and, 
- Will foster after-connections among the workshop members (without funding, of course);

Then you've got what you need to run a great workshop.

Workshops can be effective for synthesizing new ideas and tackling common pain points. They are expensive (in many ways), and often unnecessary. If the "right people" have other venues to meet each other and have acquired enough inter-personal trust to open up remotely, then they are ready to "gather" on the internet/teleconference.

\section{The NSF and NIH each spent a billion dollars funding science workshops last year, and all you got was a lousy white-paper.}

Significant scientific funding and scientist participation in collectives can already be evidenced in the activity of hosting scientific workshops to address important, shared issues. Science workshops are a major current expression of the value and need for science collectives. Workshops are where scientists gather in place to collectively respond to challenges they face in their research. In the future, open collaborative networks will be able to spin off virtual workshops on the internet at any time without additional funding. Today, workshops are the $20^{\text {th }}$ Century model of how to gather to solve mutual complex problems.

It is likely that you have travelled to and participated one or more workshops over the past decade. You've met a lot of really smart people. Shared gallons of really bad coffee. Had more than a few beers after long, long days of somewhat-facilitated work. You have spent considerable time helping write reports and white-papers. Most of these papers you never saw again. A few got published. Some workshops are more successful. Some are a shambles. As a mode of collective science, there are times when a workshop makes perfect sense, and maybe always will.

\section{Workshop worries}

There is also a way-and good reasons-to make the great majority of workshops unnecessary, by funding and building science communities instead. Just as digital journal articles have acquired their granularity and an arbitrary scarcity based on the history of printed journals, workshops have acquired their own granularity and scarcity. Here are some of their limits:

- Workshops need to have enough "work" to do to fill 1-1/2 to 2 days of effort (to justify 2 days of travel). You can't do a half-day or, say, a twenty-day workshop;

- Workshops need to support say 16-34 participants, and these scientists must be available at the same time; 
- Workshops get funded to explore science research topics "important" enough to justify their $\$ 40 \mathrm{k}$ budget. Other collective issues and needs are not currently very fundable.

- Workshops need to have a topic that is still an issue months after the proposal submission.

- Workshops require moving people around in airplanes.

- Some fraction of workshop proposals don't get funded at all.

Workshops are a product of Twentieth Century science. Science before the internet. Science before someone figured out how to let scientists create their own collectives online at virtually no cost.

That's right NSF and NIH funders; there is a way you can support thousands of selforganized online workshops with a net marginal cost of zero. Well... zero, that is, after investing about $20 \%$ of the current outlay for workshops to support several dozen selfmanaged science communities.

Above, we explored a working model for this Twenty-First Century strategy, building a commons with online collaborative networks. We have lessons already learned and ready to be copied across other research domains; a model that already supports better, more effective, and more nimble collectives than the current workshop model. That being said, you might be in a position (funded) to do a workshop. So do are really great one, where the knowing conversations flow.

\section{Ten rules for a better workshop}

An open-science workshop takes demand sharing and fierce equality into a two-day converse-a-thon, where every participant gets their say, and the entire room moves at the speed of conversation.

\section{Here is the Santa Barbara Workshop model:}

This is a time-tested model for getting the people-in-the-room to explore all of their expertise and their imaginations. It works every participant to their limit. The feedback most received from these workshops: "I've just worked harder and I've also had more fun than I have ever experienced before at any workshop." At the end, people actually complain of "brain fatigue," a condition you help cure with beer, or a good walk on the beach (it was born in Santa Barbara, after all). If you don't have a beach, then an up-town walk also works. 


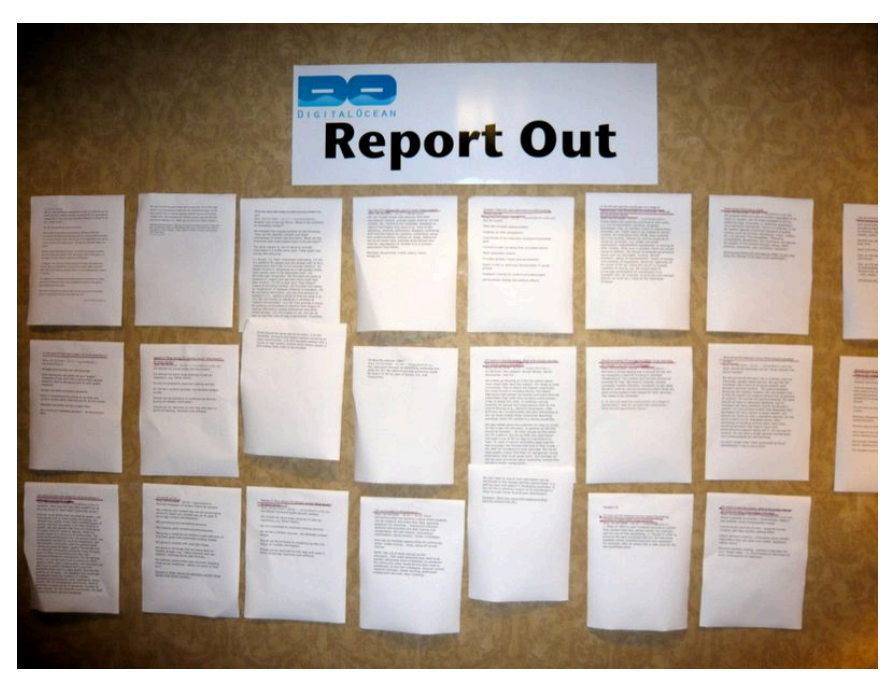

If you are interested in doing your own Santa Barbara Workshop, you can use these 10 rules to facilitate the best workshop you've ever done:

RULE 1: Pick a place that's right in town and give them dinner/lunch

Before the workshop starts, make sure you feed the participants. Pick a downtown hotel near cafes and bars. Never do this at an airport hotel, or isolated venue. If your workshop starts after lunch, feed them a good lunch first. If your workshop starts in the morning, feed them dinner the previous night. But do not try to gather them for breakfast before the workshop. People have a variety of breakfast desires. Have a table with coffee and snacks in the room.

\section{RULE 2: The ideas need to travel at the speed of conversation}

No more than $35 / 36$ people. Small groups all day.

The workshop planning should focus on getting a wide spread of expertise in the room, but no more than about 35 people ( 7 tables of five, or 5 tables of 7, or 6 tables of 6 ). The whole day will be used to promote critical conversations at these tables. As soon as the conversation lags at a table, give it something new to do (e.g., another question [see \#4 below]).

\section{RULE 3: Open with a blue sky session, get the creative juices going}

Start the conversations with a real "blue-sky" design problem. Let everyone add their fantasy to the solution of a problem. Give them paper and markers, scissors and glue. Give them props and tape. This is the only session where there is a brief report out. Let the groups compete for the most fantastic solution. Have them map their ideas on big Post-its and then stick these on the walls of the room. This beginning session is designed to help the group achieve an open conversational mode of interaction.

\section{RULE 4: Give them real questions to answer, and let them add to these}

After the blue-sky exercise, each table is given a question to tackle (not necessarily the same question, although most tables might end up answering every question). In the weeks before the workshop, spend real time coming up with 10-12 key questions. 
Map out how the answers to these add up to a larger picture. Rank these questions as "central" or "if time allows". Create some colored sheets of paper that say "Hot Topic" on them. Give each table a few and encourage people to create their own question. Give these questions to OTHER TABLES. Never let someone answer their own question. Some questions will be better answered by tables with specific expertise, others by tables of mixed expertise (see below). Credit: This rule was provided by Susan Colitan, Vice President of the Paul G. Allen Family Foundation. The better the questions the more knowledge you will extract from the workshop!

\section{RULE 5: Break up groups 2-3 times over the course of the day and vote with your feet}

Give each member a name tag (first NAME on both sides). This tag should also tell them which table at which to sit (designated by color, number, animal, etc.). You might want to start by mixing up the expertise at each table. For example the COLOR designated tables might include a technical expert, a managerial expert, some content domain specialists, and others. After a couple hours, have everyone switch to the NUMBER table, which might be grouped by expertise. Later, they might switch to an ANIMAL table, etc. At the end of the day have a final question back at the original table. At any time anyone can move to a different table. This is called "voting with your feet." Announce this at the beginning and also every time your swap out table designations.

\section{RULE 6: Use big paper Post-its to gather ideas}

The table conversations need to be captured first on big Post-its. Have the table choose a recorder. All comments are written down. This means that each person's contribution is captured and made visual for the table. Do not simply write these on a computer. Sometimes the person who made the comment will want to revise this, or expand on it. Sometimes the recorder will not understand the comment, and will ask for clarification. Everyone's voice is heard in this process. The conversation moves as fast as people can talk. When silence breaks out, the facilitator will come by to ask if the table needs another question.

\section{RULE 7: Create narratives from the Post-its and put these online immediately}

Have a volunteer at each table who merges the contents of the big Post-its into a narrative. This narrative might be one paragraph, or several. You can capture these narratives in any way that works for you. Google documents, shared Dropbox: whatever you are most familiar with. The Post-its and these narratives are the output from the meeting. They are the gold you have woven from the ideas of your participants. It is tempting to skip the Postit and go right to a computer. Do not allow this. The Post-it step is there to keep the conversation flowing and the let each person know their ideas are being captured. 


\section{RULE 8: Many conversations in one room}

Workshop planners often make the mistake of having a plenary room and then breakouts in separate rooms. Set up the main room in round tables; all the conversations will happen there. It will get loud, but people will also gain energy from the buzz in the room. And when they are voting with their feet, they only need to meander to another table and join the conversation.

\section{RULE 9: No long introductions. No formal report-outs, but quick checks. No breaks, break anytime}

At the start, have each person stand and say their name. Then have them give three words that express their hopes for the day. This should take only 10 minutes. During the course of the day, have a volunteer print out the narratives from the tables and post them on the wall near the coffee in real time. Let the display of these become an ongoing marker of the accomplishments of the workshop.

Do not have any special report-out breaks, this only slows down the conversation. Do not schedule coffee or other breaks (except for lunch if you started in the morning), but encourage everyone to take a break any time they feel like it. They can get coffee, walk around the block, and do whatever they need to gather their attention back to the workshop. Once an hour, the facilitator will do a quick check-in with the room. Stop the conversations briefly to ask if there are any concerns about the process, and remind people to go look at the report-out wall. Arrange a good lunch for everyone.

\section{RULE 10: Facilitator keeps the conversation going}

The Santa Barbara Workshop is a fast-moving symphony of conversations and inspirations. The key is to keep the ideas flowing, capture these as effectively as possible, and support each table with a supply of questions and a recording mechanism. The main facilitator will walk among the tables ready to supply a new question, or to gather the "hot topic" questions for other tables to answer. The facilitator will also decide when to rotate the tables, and can help keep the process on track.

At the end of the day, be prepared for the participants to be excited and exhausted. They will feel like their ideas have been heard, and their contributions have been saved. When they browse the report-out display, they will see how their table's answer to the questions exposed different solutions from those of other tables. They may want to be alone after eight hours of constant conversation. They might be ready for some beer.

At the end of the day, you will likely have a document that is hundreds of pages long, with multiple insights into the key questions that your organization faces. You will have mined 
the best ideas from 35 people. And these 35 people will leave the workshop satisfied that their time and their expertise has been well used and honored.

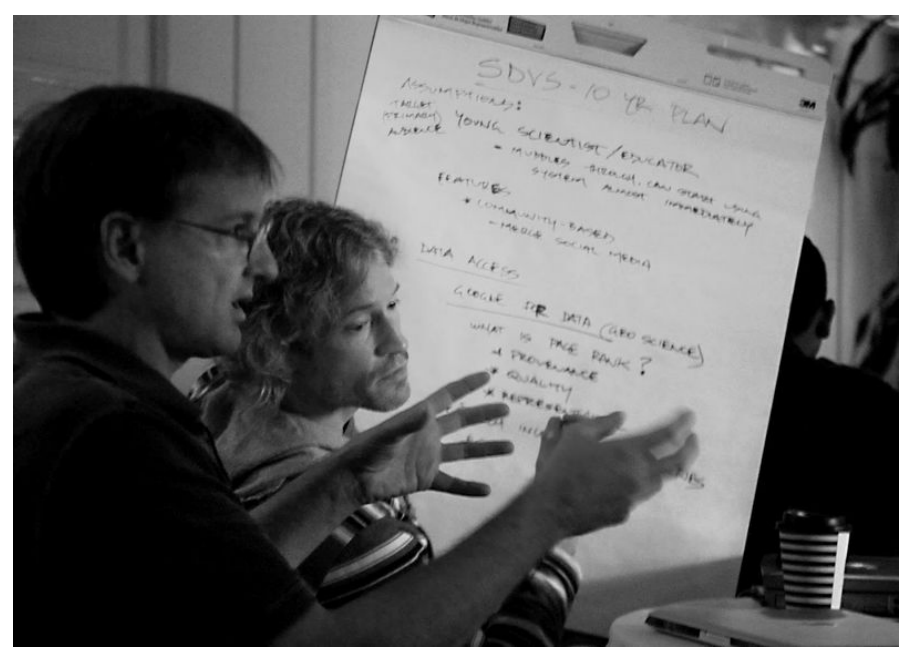

\section{My brain hurts}

The Santa Barbara workshop can be used for a wide range of planning and design problems. You can do "miniworkshops" with two or three tables. There are other similar styles of workshop, you can look them up on the web. When you ask anyone who has participated in this type of workshop, they will tell you how much fun they had getting their brains picked clean. They might also note that other workshops, where they are forced to watch PPTs in a room with 100 others, and then raise their hands one-by-one to speak, now seem boring and inefficient. This is the downside of the Santa Barbara Workshop: once you've gone there, you can never go back. 


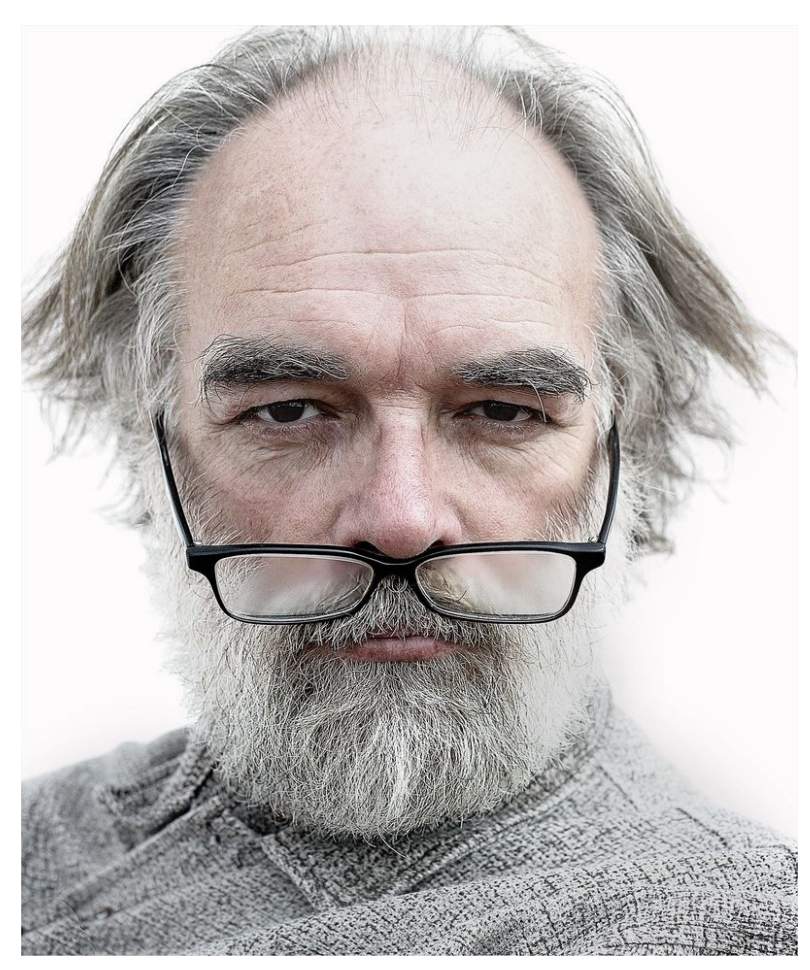

Yes. We are talking to, and maybe about, you. Image credit: Pexels on Pixabay CCO.

"What would happen, for instance, if the university were to let go of the notion of prestige and of the competition that creates it in order to better align its personnel and other processes with its deepest values? Could those institutions begin, individually and collectively, by rejecting the absurdly metrics-focused line they've been cudgeled into by the accrediting bodies that oversee them?"

(Fitzpatrick 2019).

\section{Open science heals toxic culture}

Right now, the academy finds itself in the state where failed academy institutions need to move beyond wondering how it all went so wrong, and start answering questions about their toxic cultures:

- How did university teaching become a miasma of grossly underpaid adjuncts, softmoney science staff, and administrative bloat?

- Why does doing research mean spending four months of every year writing grant proposals for programs that have an $18 \%$ success rate? 
- How come hyper-competition is allowed to dominate what should, instead, be a hypercooperative endeavor? This is science, not boxing.

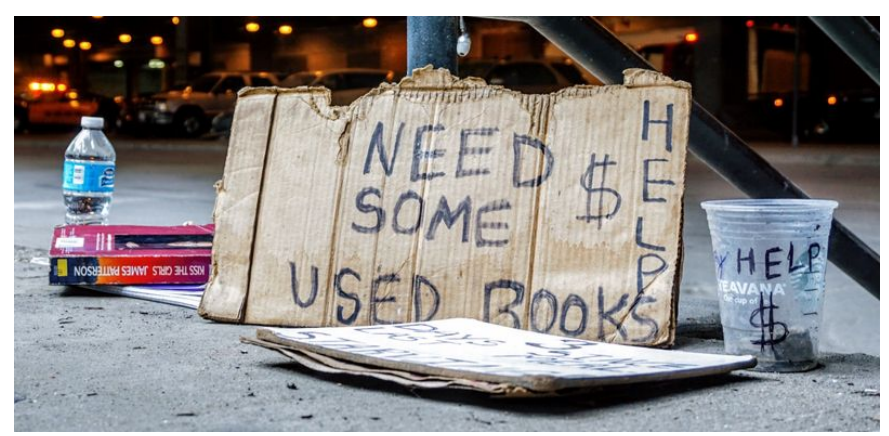

- When did higher education become a ponzi scheme for the financial sector: where government spending over the next decade will be dwarfed by the trillions in additional student debt owed to big banks (This is a USA-centric condition)? Students should not have to become servants indentured to the financial sector of the global economy just to finish their education.

- How did we arrive at an academy where sexism is so pervasive that a large percentage of women simply leave instead of facing a lifetime of symbolic violence and professional hostility? Karen Kelski (2019) created an open Google Doc (Accessed May 13,2019 ) where academics can self-report incidents of sexual harassment. More than two-thousand entries later, it is a testament to the current toxic situation in the academy.

\section{Culture repair}

Three underlying, systemic conditions that are intrinsically toxic, and which challenge other cultural practices in the academy, are well described by Yochai Benkler's (2016) discussion of "dimensions of tension;" (Accessed August 7, 2020):

"The first [tension] is the concern with the power of hierarchy; the power within an organization to be controlling...

...A second is the concern with the power of property... [T] he problem is, of course, that property is always an organization force for oligarchy; the re-creation of power around who owns it...

And the third... is the tyranny of the margin. The need to constantly compete in the market and find yourself in a context where you have to compete, you have to survive, you have to return returns-on-investment; and this ends up postponing the ethical commitment, because you can't live with it...." 
So this tyranny of the margin is a real constraint that we have to find a way how to break out of."

These three tensions are addressed by open science efforts through cultural practices that reassert core science norms: 1) fierce equality, which subverts hierarchy; 2) Demand sharing, which opens up the scholarly gift economy, and; 3) Kindness, Culture, and Caring: creating cures for the affective damage of the neoliberal academy.

The sources for the toxic damage to cultures within the academy are multiple and their impacts global. Almost any organizational culture will go toxic if simply ignored; cultures in the academy have also been warped through intentions. One noticeable result is an old nemesis: jerks in charge, or just in the room. Bad actors in the academy may rely on status quo organizational norms and rules to support their behaviors. A non-reflexive, internallyconflicted cultural milieu gives them cover for their self-promotion. They can become powerful adversaries to culture change. This condition is so common, it has its own chapter in the Handbook: The Zero-Asshole Zone.

Since toxic culture often supports and even applauds bad behaviors (centered around selfpromotion and a lack of empathy), toxic organizations actually grow jerks (by allowing jerkish behaviors) internally over time. These assholes-of-convenience may be happier when the organizational culture becomes post-toxic. Authentic bad actors bring their own more durable personality problems to the table.

Jerks are also what Grant (2013) calls "takers." Takers gobble up resources without contributing their share: "Takers have a distinctive signature: they like to get more than they give. They tilt reciprocity in their own favor, putting their own interests ahead of others' needs. Takers believe that the world is a competitive, dog-eat-dog place. They feel that to succeed, they need to be better than others. To prove their competence, they selfpromote and make sure they get plenty of credit for their efforts."

Takers are easy to spot in asynchronous internet discussions; they offer little and demand much. Once you are certain that these people are bad actors they might get added to your own memory/list of toxic weak-tie associates, and can be avoided (delisted, blocked, etc.), and others can be warned about them. Gossip within the academy can offer a low-cost corrective to bad actors (either confirming other's perceptions, or creating back-channel opportunities for rethinking one's own evaluation of the "bad actor" (See: Feinberg et al. 2012).

Of course, not all scientists who are opposed to organizational change are bad actors. They may simply see the current system as having worked well for them, and hold the opinion that change might not lead to a better working situation. They might prefer their current 
situation, however toxic, over one that is more experimental, as all new cultural endeavors need to be. Benkler's observation about "postponing the ethical commitment" can be evidenced by a consequent deluge of bullshit in the academy.

\section{On becoming a scientist: escape from bullshit}

"A scientist who is not concerned with the reproducibility of their experiment is quite simply, and somewhat paradigmatically, a bullshitter" (Frankfurt 2009).

When a scientist steps up to infinite play with her chosen object of study, there is no space, no incentive, no possible reward, for inauthentic actions. Rigor, transparency, insight, diligence: these are all marks of a congruent mind bound to the mystery it must resolve. This is not to say that scientists are necessarily saints. But rather, that the part of them that is most integral to their research must be congruent in the effort. Everything else is bullshit.

Under the logic of neo-liberal economics, where conflicts of interest intercept science practice, some scientists will cheat on the truth. When a scientist, or team of scientists, cannot own up to their failure, then; "the lack of a variety of knowledge sufficient to know what a truth might entail means that the whole enterprise becomes a project of bullshit..." (Frankfurt 2009). One of the goals of open science is to remove as many conflicts of interest as possible. Just as open science organizations need to be asshole-free zones, they must also strive to become bullshit-free research endeavors.

There are other sources of baloney in the academy, mainly from sloppy analysis, poor visualization, and lazy, biased thinking. Calling Bullshit, a class at the University of Washington (Accessed April 14, 2019) spends a whole term examining various aspects of this. Kathryn Schultz notes: "confirmation bias is entirely passive: we simply fail to look for any information that could contradict our beliefs. The sixteenth-century scientist, philosopher, and statesman Francis Bacon called this failure 'the greatest impediment and aberration of the human understanding,' and it's easy to see why. As we know, only the black swans can tell us anything definitive about our beliefs - and yet, we persistently fail to seek them out" (Schulz 2011). Scientists task themselves to develop a deep, reflexive awareness of their own knowledge:

"[W] hat, then, distinguishes those who are capable of reasoning scientifically from those who are not?... [I]n order to separate theory from evidence, one must also be able to reflect on the theory as an object of one's thinking (metacognition). To coordinate and change theory to fit new and especially disconfirming evidence one must be able to stand back from one's ideas and see them as things that can and should be tested" (Feist 2006).

Disconfirming theories is another area where open science wants to develop more capacity, rigor, and cultural force. Today, hobbled by a publishing industry attuned to printing only the latest discoveries, theories may linger without examination: 


\begin{abstract}
"Science is self-correcting (Merton 1942, 1973). If a claim is wrong, eventually new evidence will accumulate to show that it is wrong and scientific understanding of the phenomenon will change. This is part of the promise of science-following the evidence where it leads, even if it is counter to present beliefs. We do believe that self-correction occurs. Our problem is with the word 'eventually' The myth of self-correction is recognition that once published, there is no systemic ethic of confirming or disconfirming the validity of an effect. False effects can remain for decades, slowly fading or continuing to inspire and influence new research.... Further, even when it becomes known that an effect is false, retraction of the original result is very rare.... Researchers who do not discover the corrective knowledge may continue to be influenced by the original, false result. We can agree that the truth will win eventually, but we are not content to wait" (Nosek et al. 2012).
\end{abstract}

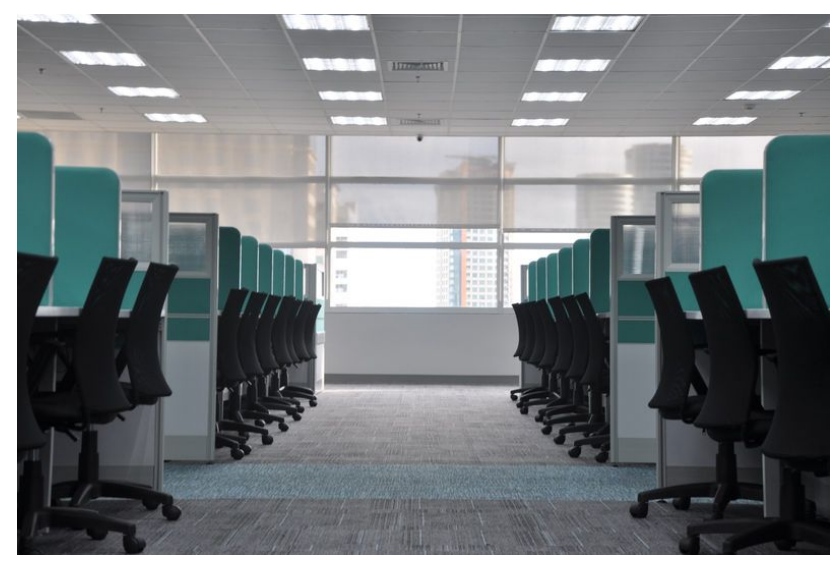

Whatever you do, don't sit down here.

The academy is also plagued with what David Graeber calls "bullshit jobs:" "Consider here some figures culled from Benjamin Ginsberg's book The Fall of the Faculty (Oxford, 2011). In American universities from 1985 to 2005, the number of both students and faculty members went up by about half, the number of full-fledged administrative positions by 85 percent - and the number of administrative staff by 240 percent....Support staff no longer mainly exist to support the faculty. In fact, not only are many of these newly created jobs in academic administration classic bullshit jobs, but it is the proliferation of these pointless jobs that is responsible for the bullshitization of real work..." (Graeber 2018; Accessed April 14, 2019). 


\section{UCLDH seminar: 'Excellence is Bullshit'}

www.ucl.ac.uk > UCLDH > Events *

In this sense "excellence" in the way we use it in the academy is a textbook example of bullshit.

Cameron Neylon will argue that the rhetorical and political power of excellence as a concept is a resul not of any imposition from the outside by administration or government, but of the stories we academics tell ourselves.

\section{Excellence is Bullshit Cameron Neylon - SlideShare}

https://www.slideshare.net/CameronNeylon/excellence-is-bullshit $\mathbf{v}$

Oct 26, 2017 - One variant of the "Excellence is Bullshit" talk about evaluation, rankings etc. Builds on

"Excellence R Us" Moore et al Palgrave Communications, 2017.

\section{Excellence is Bullshit: talk by @CameronNeylon at \#UCDavis (with ...} https://storify.com/phylogenomics/excellence-is-bullshit v Sep 13, 2016 - A Social Media Story storified by Jonathan Eisen.

Finite games are rife with bullshit metrics. The poster-child for these is "excellence".

Finally, there's a whole layer of ersatz prestige that the academy has built up around what Cameron Neylon and others call "bullshit excellence;" "Excellence"” they remind us, "is not excellent, it is a pernicious and dangerous rhetoric that undermines the very foundations of good research and scholarship" (Moore et al. 2017).

All these sources of bullshit in the academy are why open science needs to be based on fierce equality. Fierce equality will prompt significant changes to how societies, universities, and funders view and support scientific endeavor. Fierce equality militates against bullshit excellence and privilege in the academy, against the gamification of careers and reputations using external metrics, such as journal impact factors, and ultimately against all forms of the "Matthew effect" that amplifies inequality in funding and recognition. Fierce Equality - together with Demand Sharing-enables open scientists to pursue their passion for new knowledge freed from the conflicts of interest that postpone moral decisions and lead to bias and bad science.

Toxic culture can consume your organization. It's up to you to not let this happen. 


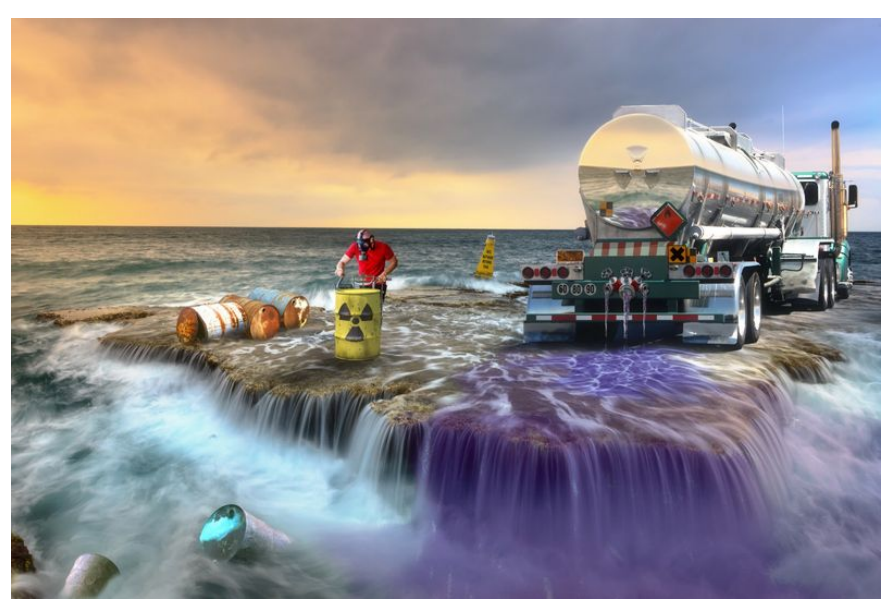

\section{Ways forward}

Toxic culture practices are not integral to how your department, laboratory, school, college, university, agency, etc. operates. You can identify these and work to end them. This is where alternative, intentional cultural practices are essential. As an open-science culture change agent, you can help grow a new organizational infraculture to dis-place and re-place toxified practices. It takes work to maintain a non-toxic organizational culture; but a lot more work to fix one that has become toxic - not to mention the cost at the individual level in terms of lost potential. How do you keep your organizational culture positive, transparent, and democratic?

"The most hidebound, bureaucratic, lumbering, terrible organization got each one of its unreasonable policies one drip at a time. Taken individually, each layer of stupid rules was almost weightless, but in quantity, they're a smothering weight." (Cory Doctorow (2014); Accessed June 25, 2020).

\section{Learning from Silicon Valley}

The practical advantages of stewarding your organization's culture so that this does not drift into toxic shallows are now topics of hundreds of start-up organizational guiding books, articles, blogs, conferences, and consultant efforts. CEOs are routinely coached about the necessity of "not fucking up your culture," (2014 blog by Brian Chesky, CEO of AirBnB; Accessed October 5,2020 ). An equal number of guides outline what people generally know: how to recognize when your organization's culture has gone sour. You can Google up "what does toxic culture mean" and get a couple hundred million pieces of advice. A lot of the conditions that are described as toxic at the organizational level resonate with behaviors that are attributed to assholes at the individual level.

\section{Institutional guilt}

One major example of toxic management practices are institutional rules that everyone knows, but few follow. This situation leads to "institutional guilt." You are still guilty for not following a rule that nobody follows. 
Institutional guilt happens when values and vision, and policies and processes are routinely broken. The routine creates an alternative policy, a counter-value, which becomes the operational norm for the organization. As this new policy and its values cannot be spoken of, it is almost impervious to change. The "real rule" is unspoken, detached from official management practices, and cemented into everyday interactions at the organization.

In a mostly volunteer organization, like a learned society, or an online network, this situation will lead to almost certain failure; volunteers will flee, staff will be disengaged. In a university department or school, employees will look for lateral transfers to less stressful units. Those that remain may do so for suspect reasons. Bullies and jerks take advantage of institutional guilt by creating a show of following the written rules and threatening those who don't.

Institutional guilt grows from a routinized violation of your organization's stated values, vision, rules, or policies. When enough people violate a rule without effects, the rule does not just go away. The rule becomes weaponizable as a tool to arbitrarily punish individuals. Institutional guilt is symptomatic of dysfunctional communication strategies inside an organization. It leads to distrust of staff and disengagement from the organization's vision. Staff and volunteer disengagement/disenchantment is a prime reason organizations fail (Duckles et al. 2005). Your university needs to escape this all-too common vortex of toxic behaviors. Institutional guilt is something that will ruin your academic institution or online organization. It poisons the culture and it drives away volunteers while it demoralizes your staff.

The routines that include institutional guilt are a subset of what Rice and Cooper (2010) call "unusual routines." These are dysfunctional outcomes, mostly of flawed communication practices. There is no organizational structure that can prevent these entirely. There are cultural practices available to repair unusual routines. The notion that toxic culture fosters jerks is only a part of the dynamic. The emboldened assholes do their part to add more toxicity over time. One of the remedies for toxic culture is to marginalize those jerks who are a) unrepentant, and b) un-fireable (e.g., assholes with tenure).

In the process of introducing the virtues and values of open science, you might (and should) look to see how these dis-empowering jerkish tactics operate in your team, department, or laboratory.

As opportunities and incentives for bad behavior diminish, you might discover that colleagues who behaved badly in the past-perhaps including you, on occasion (getting a $\mathrm{PhD}$ opens up a lot of doors for bad behavior) - no longer feel like they have the need to be unkind to one another. Change the culture and you might also help others internalize the new virtues your culture promotes. 


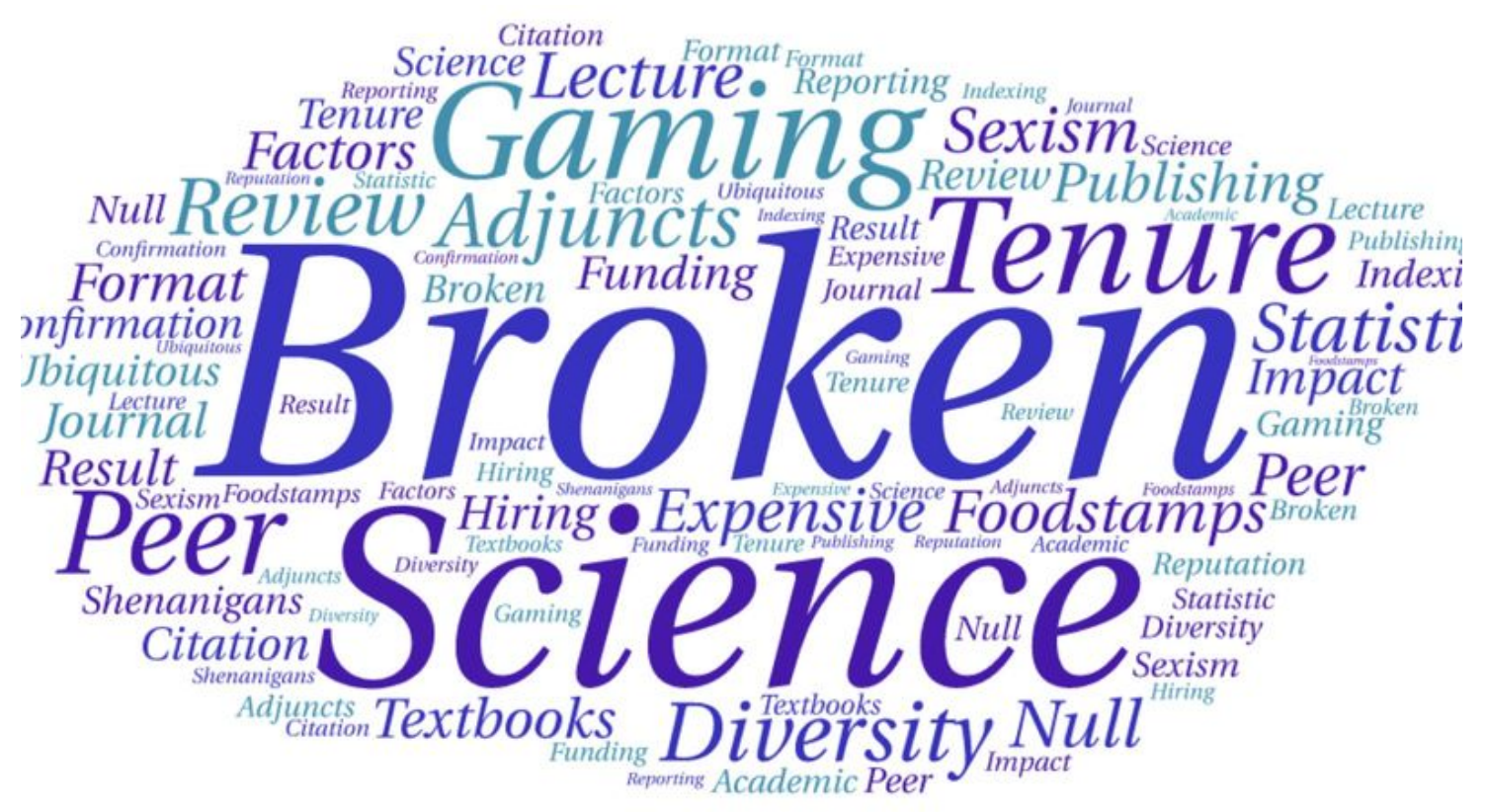

Toxic cultural practices will murder your academic dreams

Let's recap here:

1. Hyper-competition feeds self-interested behaviors and can lead to sadism, or, more commonly, to efforts at manipulation (faking data, taking credit, undermining the efforts of others), and harassment of junior colleagues. Other people become obstacles. They exist to be defeated/demeaned or to become instruments to use in the process of "winning."

2. Sexual harassment and intellectual bullying feeds spitefulness in those who survive this, and who later find themselves in a position to bully others. Spitefulness also infects the peer-review process for publication, employment, career advancement, and research funding.

3. Bullshit prestige and the Matthew Effect feed entitlement, narcissism, and egoism among those in career positions that have benefitted from the process of cumulative advantage. They use their advantaged position to promote more bullshit prestige.

4. The neo-liberal market logic feeds moral disengagement, delaying moral decisions in favor of expedience and advantage. Cheating on research results to gain publication harms the entire academy.

5. Organizational culture decays from neglect and the intentions of bad actors within. Institutional guilt replaces transparency and silences conflict. Workplaces become fearful 
and employees unproductive and unhappy. There's a whole other blog here about how the day-to-day emotional work of navigating these toxic workplaces gets offloaded to assistants and low-paid staff, often women.

\section{Your organization can govern its way out of any toxic culture}

There are some roads that lead away from toxic practices in the academy. One of them is through new governance practices. Sometimes toxic practices get burned into the everyday life of an organization. Implementing corrective governance is a good way to build in protection against institutional guilt. You also need to be sure that your employees and volunteer committees do not fall into the trap of violating your own values and policies for some immediate purpose. Better governance opens up learning capabilities and communication channels to help limit and repair occasions where volunteers or staff do stray from your organization's vision and values.

The next section of the Handbook will take you through the process of refactoring your organization using a form of "double-loop" governance. This is one way forward. There are others you can try. The point is, you are not trapped in a toxic culture, run by assholes who get to decide your professional life for you, or the front path for science.

Remember that decisions that don't get made by the people who are supposed to make them get made anyhow by the people who need them. Even the decision not to decide today is made by someone. When decisions are guided by the values and vision of the organization, when the process is transparent, when the conflicts appear on the surface, when failure is just another chance at success, and when leadership opens up in front of those who have proven their worth: that is when institutional guilt has no purchase on the logic of your organization. 


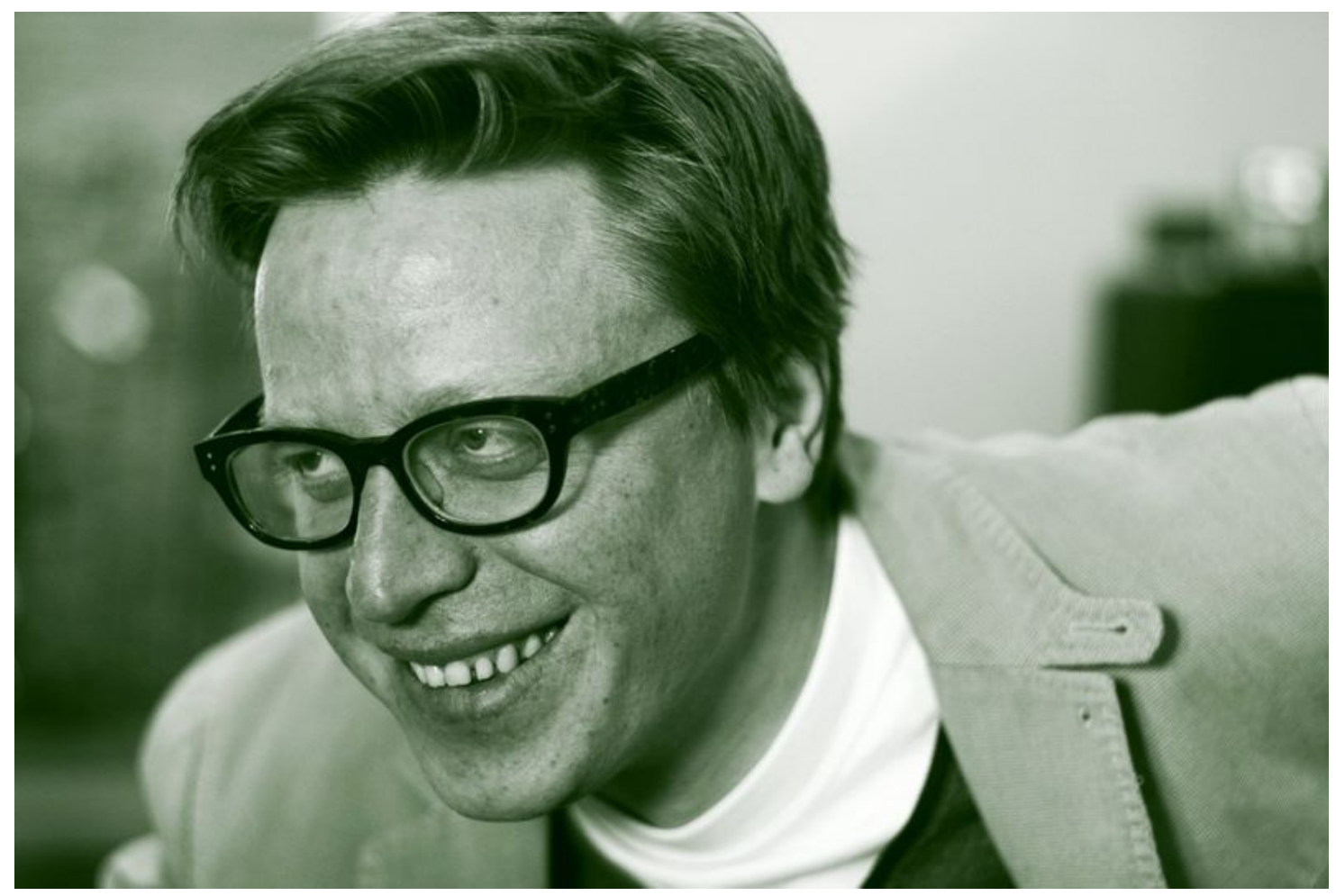

All it takes is one asshole to ruin you life. Photo Credit: Igor Pavlov on Flickr CC licensed

"First: the asshole helps himself to special privileges in cooperative life; second: he does this out of an intrenched sense of entitlement; third: he is immunized against the complaints of other people." Aaron James: Assholes: a Theory... the intro video (2012) <https://youtu.be/d2y-pt0makw>

"An asshole is someone who leaves us feeling demeaned, de-energized, disrespected, and/or oppressed. In other words, someone who makes you feel like dirt" (Robert Sutton 2019; Accessed April 9, 2019).

\section{The Zero-Asshole Zone}

\section{Your organization can make this happen}

Depending on who you talk to, the academy's asshole problem is either extremely dire, vastly complicated, or both. Very few people would say it doesn't exist. The "complicated" version tries to balance assholish behaviors with some idea that the pursuit of new knowledge in a hyper-competitive environment requires a intellectual with an enhanced sense of self-confidence, an enormous ego, a thick skin, and relentless drive. Only a 
complete narcissist can out-compete all the other assholes in the struggle for resources and credit. Colleagues who hang around this social-black-hole personality hope to ride along in the car of his success (i.e., "He may well be an asshole, but he's our asshole”):

"The traits associated with narcissism explain why some people have an innate ability to dominate the scene. This includes the good serious face that implicitly tells their entourage that their research is important but also their willingness to use resources without any scruples or any sense of a possible cost for the community as a whole. This provides advantages in a system that monitors production and not productivity. We can understand why these innate leaders have supporters that praise their qualities - because of their fast-track access to resources that are usually difficult to get" (Lemaitre 2015).

This "nice scientists finish last" mind-set serves to demonstrate why open science, with fierce equality and demand sharing, is an important, and urgent, remedy for the academy. The "cost" to the community-and to your own team, lab, department, or school-of even one real asshole is greater than you might at first guess. Assholes breed more assholes as they chase away nice, clever people. "Ultimately we are all diminished when clever people walk away from academia. So what can we do? It's tempting to point the finger at senior academics for creating a poor workplace culture, but I've experienced this behaviour from people at all levels of the academic hierarchy. We need to work together to break the circle of nastiness" (emphasis in the original) (Mewburn 2015; Accessed April 9, 2019).

\section{You can argue ideas without being an asshole}

It is really important here to understand that arguments over ideas are not intrinsically assholish events. As we will see below, assholes demean other individuals; their behavior is aimed at people. They will also be abrasive and demeaning in the manner in which they defend their ideas. We've all witnessed this in conferences and seminars. Entire paragraphs of meeting "code of conduct" rules are meant to counteract this kind of behavior. Sutton offers this: "enforcing a no asshole rule doesn't mean turning your organization into a paradise for conflict-averse wimps. The best groups and organizations-especially the most creative ones-are places where people know how to fight" (Sutton 2007).

Another complication the academy has is this: assholes with tenure. Sutton has no clear answer for this problem: "I'm with all these colleagues that are all tenured, and Stanford has no mandatory retirement,' he points out. 'So when I'm with an asshole, all I can do is hope"' (Sutton 2017; Accessed 4/9/2019; emphasis in the original).

"Science advances one funeral at a time;" Max Planck (1932/2015) had other, grand theoretical, reasons to say this. It also applies to assholes with tenure. So the best thing to do is: never hire an asshole in the first place. This is the essential message of the No Asshole 
Rule. No matter how much of an academic star she/he might be, adding him/her to your faculty is a huge mistake, even more so when they show up already with tenure.

In a corporate environment, you can just ask a high-powered jerk employee to go be a jerk in some other corporation. CEO coaches offer a simple principle: "'genuine collaboration and accountability for our own actions are non-negotiable if you plan on succeeding in this place'. ... Get this right [as a CEO], and you will set yourself up with a culture that delivers far greater and more consistent long term success than the short term spikes delivered by a Jerk!" (Francis 2017; Accessed April 9, 2019).

Assholes in positions of power in your organization can be sidetracked as much as possible, isolated and ignored as circumstances allow. Graduate students can be warned away, administrators can be informed, and professional associations - where these assholes are eager to get into leadership positions-can be immunized through active word-of-mouth. Remember that a single asshole can impact your organization for years.

In The Problem with Assholes, Elizabeth Cullen Dunn announced that "Anthropology has an asshole problem." She notes, "[a]ssholery is contagious. Once people see an asshole being an asshole and winning, actually gaining power and prestige by being an obnoxious selfinterested bully, it creates a huge incentive for other people to emulate that behavior. Assholery has ripple effects as it spreads in the form of disciplinary norms that not only enable, but hyper-value nasty, elitist, demeaning behavior" (Dunn 2018; Accessed April 9, 2019). Anthropology is not alone. In a 2018 report, the National Academies note: "In a survey conducted by the University of Texas System..., about 20 percent of female science students (undergraduate and graduate) experienced sexual harassment from faculty or staff, while more than a quarter of female engineering students and greater than 40 percent of medical students experienced sexual harassment from faculty or staff" (NAS 2018). The asshole problem is acute across the academy.

\section{Situational assholes}

Sutton (2018 and 2007) notes that, on occasion, anyone can act assholishly. These "temporary assholes" are not the real problem. They tend to want to repair their lapses of civility, and to feel bad about their own behavior. The real problem comes from "authentic assholes." Authentic assholes display durable personality issues, and are also more likely to engage in an "exploitative sexual style" (Jones and Figueredo 2013) that seeks instrumental sex with multiple partners; a trait that powers workplace harassment.

There is also a subset of assholes in the academy who are "accidental assholes" (epah 2017; Accessed April 9, 2019). These are nerdish individuals who are, for example, on the autism spectrum, and who do not have the social skills to always act appropriately. They may do randomly assholish things, or they may simply copy the bad behaviors they find 
around them. Accidental assholes are still jerks. And being on the spectrum is no excuse. "It's a conscious choice, no matter how much lead-in, to become like that," Brandon Weaver (2019; Accessed November 10, 2019) explains.

Not all assholes are born that way: lots of them are nurtured into bad behaviors on the job. The current, toxic academic culture can turn a temporary asshole into an chronic bad actor, a kind of "opportune asshole;" (or, in evolutionary culture terms, an "adaptive asshole"): someone who believes that bad behavior is expected of them and rewarded by their peers. They are happy to oblige.

This may be why so many precincts of the academy seem to be swarming with assholes (jerks, bad-actors, etc.). When you add the opportune- and temporary-assholes to the authentic ones, the numbers and their bad effects really add up. Sutton addressed this situation in an article in the Harvard Business Review (<https://hbrorg/2007/05/why-arethere-so-many> Accessed April 9, 2019). As the National Academies found, the most asshole-infested profession is medicine and medical school:

"A longitudinal study of nearly 3,000 medical students from 16 medical schools was just published in The British Medical Journal. Erica Frank and her colleagues at the Emory Medical School found that 42 percent of seniors reported being harassed by fellow students, professors, physicians, or patients; 84 percent reported they had been belittled and 40 percent reported being both harassed and belittled" (Sutton 2007).

So, why are we surrounded by assholes? Sutton explains:

"The truth is that assholes breed like rabbits. Their poison quickly infects others; even worse, if you let them make hiring decisions, they will start cloning themselves. Once people believe that they can get away with treating others with contempt or, worse yet, believe they will be praised and rewarded for it, a reign of psychological terror can spread throughout your organization that is damn hard to stop" (Sutton 2007).

\section{The who is more important than the what}

The good news is that the principles of fierce equality and demand sharing are diagnostic and therapeutic in finding and neutralizing assholes. Once the opportune-assholes find that their bad behavior is no longer applauded or even acceptable, they will need to selfmonitor their personal interactions. When open-science norms support public acknowledgement of the asshole problem, and offer remedies for this in departments, labs, colleges, professional associations, etc., authentic assholes will find that their toxic actions serve only to isolate and shame them (even though they may not feel this shame as shame, but convert it into anger (Brown 2012)). Over time, when new norms take hold, and new 
hires bring fresh non-assholic voices into the mix, your corner of the academy can regain its fundamental civility, and you and your students can again argue theories and ideas, methods and experiments, without resorting to abuse and fear.

Working in a zero-asshole environment is significantly more pleasant and productive than toiling in the psychological minefield that even one asshole can create in your department, laboratory, agency, or college. Achieving a zero-asshole status takes a principled stance and procedural follow-through. It is a worthwhile goal for you as an open science culturechange agent to pursue. "Bear in mind that negative interactions have five times the effect on mood than positive interactions-it takes a lot of good people to make up for the damage done by just a few demeaning jerks" (Sutton 2007).

\section{The asshole in the mirror}

A final thought here. Each of us is capable of astounding assholishness at any time. Most of us have experienced being on the receiving end on some occasions (in seminars, through peer review, at office hours) of abuse by those who control our academic fortunes, and use fear and humiliation in their critiques of our work, or of our capacities for research or teaching. We know how to do asshole; we've had enough training. We just need to not go there. And we need to isolate ourselves from the assholes we encounter. Sutton reminds us of this:

"If you want to build an asshole-free environment, you've got to start by looking in the mirror. When have you been an asshole? When have you caught and spread this contagious disease? What can you do, or what have you done, to keep your inner asshole from firing away at others? The most powerful single step you can take is to...just stay away from nasty people and places. This means you must defy the temptation to work with a swarm of assholes, regardless of a job's other perks and charms. It also means that if you make this mistake, get out as fast as you can. And remember, as my student Dave Sanford taught me, that admitting you're an asshole is the first step" (Sutton 2007).

You can always take Sutton's (2007) "asshole test" to self-diagnose. Or, if you find yourself believing that you are surrounded by idiots and that you should be recognized for your real talents and elevated into a higher level of society: you are probably an asshole, or at least, a "jerk":

"Because the jerk tends to disregard the perspectives of those below him in the hierarchy, he often has little idea how he appears to them. This leads to hypocrisies. He might rage against the smallest typo in a student's or secretary's document, while producing a torrent of errors himself; it just wouldn't occur to him to apply the same standards to himself. He might insist on promptness, while 
always running late. He might freely reprimand other people, expecting them to take it with good grace, while any complaints directed against him earn his eternal enmity" (Schwitzgabel 2014; Accessed April 9, 2019).

This is a good reminder that assholes know who and what to kiss to get ahead. They may direct their assholocity at anyone/everyone lesser than them in the academic scheme, and act entirely respectful and encouraging to those above them. Your dean may not know who's an asshole, but grad-students might have a clear idea. Listen to them. They may already be organizing to minimize the impacts of faculty assholes on their future careers. At the University of North Carolina, graduate students organized "Academics taking Action" for this purpose ( $<$ https://academicstakingaction.wordpress.com/> Accessed 5/14/2019). And should you, in a moment of fatigue or stress lash out at your students, if you are a temporary asshole, then it's up to you to make them know you acted poorly and regret it.

Feeling mean today? Go ahead, be mean to your data; interrogate it ruthlessly. Be cruel to your theories. Don't look to validate them, find new ways to attack them. Be an asshole with your methodology; it's certainly not as rigorous as it could be. Then, have some more coffee and be kind and humble with your students and colleagues. 


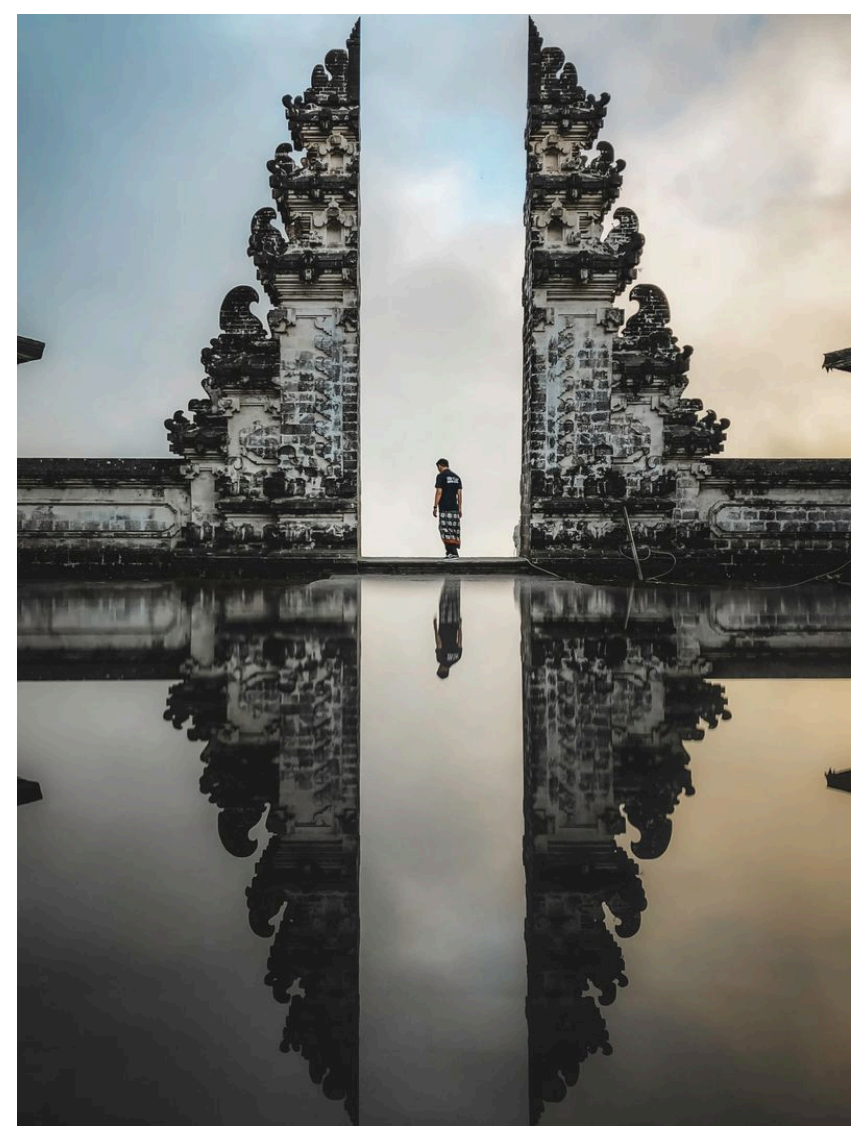

\section{The Congruent Scientist: Infinite play builds personal wisdom}

"Yet notoriously the cultivation of truthfulness, justice and courage will often, the world being what it contingently is, bar us from being rich or famous or powerful. Thus although we may hope that we can not only achieve the standards of excellence and the internal goods of certain practices by possessing the virtues and become rich, famous and powerful, the virtues are always a potential stumbling block to this comfortable ambition" (MacIntyre 1984).

"For us, activism in the academy springs from and serves the infinite game: it is action beyond the rules that calls us to take our intuitions, lived experience and observations of injustice and exclusion seriously. Academic activism aims to document, subvert and ultimately rewrite the rules of the finite games we currently live by, so that they make more sense to us as people seeking to give of our best to an endeavour ('the university') that we cannot help but believe in" (Harré, et al. 2017; emphasis added). 
Alasdair MacIntyre's caveat rings true when applied to finite power games in and out of the academy. It's the same "nice guys finish last" logic that your academic advisor may have given you; "practical" advice that many in the academy have used as an alibi against sharing their data and methods openly. Open scientists know better. This logic exposes how the external goods of the neo-liberal market are crowding out the internal goods so vital to the success of science as a practice. Nicholas Gruen (Accessed February 21, 2020) puts it this way: "Here's the serpent in paradise. External goods are necessary, but, at the same time, in tension with internal goods. This is an ethical tension. The risk is always that the pursuit of external goods compromises the pursuit of internal goods, and thus the excellence of the practice."

Certainly, during the transition phase to open practices of Fierce Equality and Demand Sharing, there will still be finite games for external goods where the few consider themselves winners, and all others as losers. The hyper-competition for scarce external goods in the academy is not going to simply disappear on its own. Certain finite game strategies can also be helpful, for sharpening methodologies, say, or microfunding opportunities. So, some finite games will continue.

Today, dozens of open science endeavors have articulated alternative solution pathways for distributing external goods without supporting perverse incentives (Edwards and Roy 2017; Bartling and Friesike 2014). However, building alternative practices through intentional culture change takes time and effort. The open, infinite game of science treasures its abundant internal goods-including internal measures of recognition and modes of compensation-above money, fame, and glory. Something to remember when you have little of the latter.

One particular internal good in the academy happens when your work and your person become congruent - the practical wisdom of doing science you learn and apply to your research, and with your teammates. You will also find this useful in your personal life with friends and family.

\section{Background on the notion of congruence}

In 1961, the psychotherapist Carl Rogers compiled three decades of papers into the book On Becoming a Person. The main frame of the book describes his client-centric approach to psychotherapy, how he arrived at this and what he learned as a practitioner. Many of the articles read very much as blogs do today (and were unpublishable in the scientific journals for this reason). He then links this frame to other human endeavors. In particular, he looks to education and personal relations in organizations.

His main therapeutic process involves how the psychoanalyst as a person develops her own personhood by becoming more congruent (more about this in a minute) and then uses this 
congruence as a communication tool to open up the client to the process of becoming more congruent. The therapist is really only someone further along the same road to "becoming a person."

The process of becoming a person, of achieving more and wider congruence, and so having fewer and fewer defenses, brings the client to a better life, with less tension and fear, better communication with everyone, and new opportunities to explore each moment fully. For Rogers, congruence happens when one's real self (the one we all start out with-all infants are congruent-also the one we can shape with our own skills and the virtues we build) fully resembles one's ideal self (the one we acquire from social interactions with others).

Another way to look at this reveals the parallels between personal change and organizational change: the person moves away from managing their fears, and attempts at controlling the behavior of others, to governing their own growth: becoming more reflexive, open to self-examination, better able to handle conflict, and by this, becoming more resilient and capable of change. The very same pathway is how organizations can successfully change (Argyris 2004). The goal of congruence is also extremely well aligned with the goal of "becoming a scientist:" congruence unlocks intellectual creativity.

\section{Creativity in science is a self-therapeutic practice}

"The mainspring of creativity appears to be the same tendency which we discover so deeply as the curative force in psychotherapy-man's tendency to actualize himself, to become his potentialities....This tendency may be come deeply buried under layer after layer of encrusted psychological defenses; it may be hidden behind elaborate façades which deny its existence; it is my belief however, based on my experience, that it exists in every individual, and awaits only the proper conditions to be released and expressed. It is this tendency which is the primary motivation for creativity as the organism forms new relationships to the environment in its endeavor most fully to be itself" (Rogers 1961).

The ideal self represents the roles you acquire in order to play finite games in your life and career. The rules you follow may still be the same rules you determined in early childhood. So by now, you are really good at following; and far less capable of knowing why you do so. For example, in your academic position, you are "The Scientist." As Carse (1987) noted and Rogers might agree here, you have forgotten that you have the freedom to set aside your role. Quite the reverse: the role has become you. This is why you spend so much effort defending your ideal self/role, even (or especially) against your real self. How do you escape?

"[P]rogress in personal life and in group living is made in the same way, by releasing variation, freedom, creativity" (Rogers 1961). The creativity you invest in the infinite game 
of science builds the capacity you can use to release your real self from the roles you play in finite games. You acquire the mindset of an infinite player and become self-directed toward congruence. As Rogers notes:

"[T]he individual who is open to his experience, and self-directing, is harmonious, not chaotic, ingenious rather than random, as he orders his responses imaginatively toward the achievement of his own purposes. His creative actions are no more a chaotic accident than was Einstein's development of the theory of relativity" (ibid).

Simon Sinek (2019) would add that your infinite player mindset is precisely what you need to succeed as a team member in $21^{\text {st }}$ Century science.

\section{Open science and an open you}

How does the authenticity of doing science as an infinite game bleed over into your personal life? Can science really make you a "better person"? The skills you acquired to become a scientist, and the cultural practices of open science you are weaving into your work are tools you can use if you bring along the courage to seek change; "making courage part of your personal culture means you are always willing to keep making changes in your life until it is the life that you want and the life that you deserve. Courage in your life means you accept that there will be missteps - that constant and repeated change may be necessary, but that it is nothing to be ashamed of if it leads to a more fulfilling, positive outcome (Dudley 2018).

The intellectual tools of science, such as rigorous, reflexive curiosity, openness, and intellectual humility are available to the scientist for use in other circumstances. You can bring these skills home with you. The same kindness and humility you bring (one hopes) to your teaching and research can become an interactive style elsewhere.

\section{On becoming an organization}

“If things aren't going right, the first response is: let's make more rules, let's set up a set of detailed procedures to make sure that people will do the right thing. Give teachers scripts to follow in the classroom, so even if they don't know what they're doing and don't care about the welfare of our kids, as long as they follow the scripts, our kids will get educated. Give judges a list of mandatory sentences to impose for crimes, so that you don't need to rely on judges using their judgment....Impose limits on what credit card companies can charge in interest and on what they can charge in fees. More and more rules to protect us against an indifferent, uncaring set of institutions we have to deal with" (Barry Schwartz 2011 TED Talk; Accessed 02/17/2020). 
In the Handbook's section on Learning Organizations, we discovered double-loop learning/ governance. Double-loop governance maps directly into congruence at the organizational level: the so-called "ideal" organization being the first loop, and the real organization being the second loop. Instead of hiding the real organization behind the idealized intentions of the founder, or top-down rules and regulations derived from an imperious CEO or provost, double-loop governance makes the real organization available to every member. Each member has the same view and purview of the rules and roles, the values and the vision of the organization, and also an obligation to make these congruent with the everyday activities of the organization. Real change for the organization also requires its membersnot just the CEO-to learn to use "valid information, informed choice, and vigilant monitoring of the implementation of the choice in order to detect and correct error" (Argyris 2004).

"Double-loop learning focuses on detecting and correcting errors in the designs of the master programs that underlie the routines. When this is the case, the attention is focused on changing the governing of the values, policies, and master programs that produced the routines in question in the first place. Double-loop learning is at the heart of the distinction, often made in the literature, between doing something right and doing the right thing" (Argyris 2004).

The notion that your organization can also be a therapeutic setting where members can learn to become more congruent may seem peculiar today, where management mainly promotes rule-governed compliance. Barry Schwartz tells us to stop already with the rules and use our everyday interactions to grow social and personal virtues:

“Rules and incentives don't tell you how to be a good friend, how to be a good parent, how to be a good spouse, or how to be a good doctor or a good lawyer or a good teacher. Rules and incentives are no substitutes for wisdom. Indeed, we argue, there is no substitute for wisdom. And so practical wisdom does not require heroic acts of self-sacrifice on the part of practitioners. In giving us the will and the skill to do the right thing - to do right by others-practical wisdom also gives us the will and the skill to do right by ourselves. (Barry Schwartz 2011 TED Talk; Accessed February 17, 2020).

Remember also that organizational "knowing" - the stock of sharable knowledge that defines your academic institution's main research resource-is a series of conversations between two or more people. Knowing cannot be stored, only generated anew as each conversation leverages the prior ones. Your organization's culture and governance sets up the circumstances at work where members can (or cannot) communicate effectively to share their insights and grow creativity. Mainly what they share is a specific, collective scientific ignorance. 
"This [the shared ignorance in a scientific conversation] is knowledgeable ignorance, perceptive ignorance, insightful ignorance. It leads us to frame better questions, the first step to getting better answers. It is the most important resource we scientists have, and using it correctly is the most important thing a scientist does" (Firestein 2012). Using open science with an infinite play mindset, you can make your organization a place where each member can become more of a person. In return they will make your organization a better place in which to get science done.

\section{Coda}

You've likely met one or more congruent scientists. Someone who has engaged you in conversation at a workshop or conference. You walk away thinking, "She is so open and intellectually humble, knows so much, and asks really great questions. She must be such fun to work with." You might have also sensed a wellspring of truthfulness, justice, and courage there. A corner of your ego might wonder what she thought of you. If you were engaged in infinite play with her, and not trying to win points, she probably noticed.

You may also have visited a congruent organization (or, with great luck, you've ended up working in one), and come away wondering how they manage to avoid all the administrative bullshit you deal with every day. How do they sustain that level of creative interactions? How come everybody felt safe enough to say those things out loud? What does one need to do to get a job there?

In the Earth sciences, there's a virtual organization called Earth Science Information Partners (ESIPfed.org). This is a great example of a congruent organization. A few years back, they hired an independent review organization to advise them on what they could do to improve. The report came back: sorry, everyone who knows you loves how you work right now. The reviewers were apologetic about not exposing problems to be fixed. ESIP has been a double-loop learning organization for two decades. It shows. Here's an introduction to their bi-yearly gatherings.

The Open Scientist Handbook offers tools for you to become more congruent and to build congruent governance practices into your own academy home. 


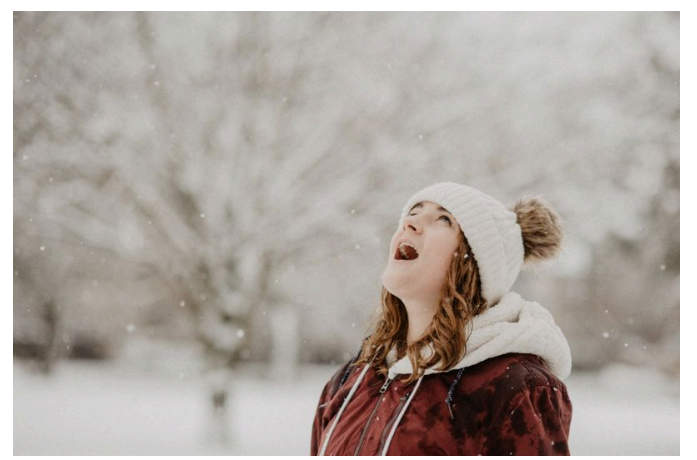

\section{Open science combines the joy of and the passion for new knowledge with a love for learning and the fun of sharing}

"It was opal and this was something I knew, something I could draw a circle around and testify to as being true. While looking at the graph, I thought about how I now knew something for certain that only an hour ago had been an absolute unknown, and I slowly began to appreciate how my life had just changed. I was the only person in an infinite exploding universe who knew that this powder was made of opal. In a wide, wide world, full of unimaginable numbers of people, I was - in addition to being small and insufficient-special. I was not only a quirky bundle of genes, but I was also unique existentially, because of the tiny detail that I knew about Creation, because of what I had seen and then understood" (Jahren 2016).

Universities are not "knowledge factories." Rather, they contain hundreds of knowledge gardens: interfaces to infinite play.

"'Garden' does not refer to the bounded plot at the edge of the house or the margin of the city. This is not a garden one lives beside, but a garden one lives within. It is a place of growth, of maximized spontaneity. To garden is not to engage in a hobby or an amusement; it is to design a culture capable of adjusting to the widest possible range of surprise in nature. Gardeners are acutely attentive to the deep patterns of natural order, but are also aware that there will always be much lying beyond their vision" (Carse 1987).

Looking at higher education at the national level in the US, every one of the 1.6 million faculty members (now, half of them part-time) adds their own knowledge resources to these gardens. By this they grow the varieties of knowing available in any one place and time: the events that allow giant ideas to emerge.

Students come to campus to acquire their own complex, emergent capacity to be "attentive to the deep patterns" of knowledge. To be clear, students also come to university to 
embrace their young adult (or pre-career) status in many ways. A lot of learning happens outside the lecture halls. The 2020 pandemic has thrown a sharp light on the value and problematics of on-campus life.

Saying the university nurtures "knowledge gardens" appears as a highly idealized notion. Granted. But it is also important to remember that without its knowledge gardens, a college becomes just another info-plantation, where low-paid precarious labor can be tasked to meet the worker needs of the information industry (sound familiar?): a higher-high school for the technically minded.

A university's knowledge gardens occupy the very center of its core value proposition: they are crucibles for new knowing and wellsprings for creativity and innovation. Additionally, there are many who are looking to carry the knowledge-garden experience down to the middle-school level (why stop there?) (Bereiter and Scardamalia 2016; Dintersmith 2018; Thomas and Brown 2011). Others would add that $21^{\text {st }}$ Century corporations need to adopt a knowledge garden-style outlook (Sinek 2019; Ito and Howe 2016; Hagel and Brown 2008).

There are those who would replace the university's knowledge gardens with infoplantations, which are vastly easier to "manage" and "improve." With some depressing regularity, the metrics for these "improvements" soon devolve into gaming the metrics themselves (Muller 2018). Applying a neoliberal, market-place logic to a university takes the worst of $20^{\text {th }}$ century hierarchical management techniques (calling these "best practices") and attaches them to faculty activities and careers, creating metric ratchets that reward additional "productivity" on a year-to-year basis.

"Universities obviously need to keep good financial books and have systems that are efficient.... But they cannot function properly as capitalist institutions. Their work of labor-intensive, craft-based creation and teaching is non-capitalist. Since capitalism will continue to insist on bottom-line measures of their output, universities will at those times need to be frankly anticapitalist" (Newfield 2016).

Of course, publicly-funded, higher education and research institutions (colleges, universities, research centers) and their sub-organizations (schools, libraries, research units) have similar personnel and resource management needs as do for-profit corporations. There is plenty of institutional work to be done, budgets to figure and stick to, deadlines to meet, facilities to maintain, the emotional labor needed to keep the workplace convivial: just like any organization.

All workers benefit when open, transparent, consensus-value-guided governance practices apply at their workplaces. But universities are not just like any organization. They cannot be managed through any set of metrics. Open science looks to break the "tyranny of 
metrics" (Muller, op cit) by expanding descriptions of the value proposition of the university (See Also: Newfield 2016; and Scarcity) to include a broad range of public goods and societal value created from the provident bounty of the academy's knowledge gardens.

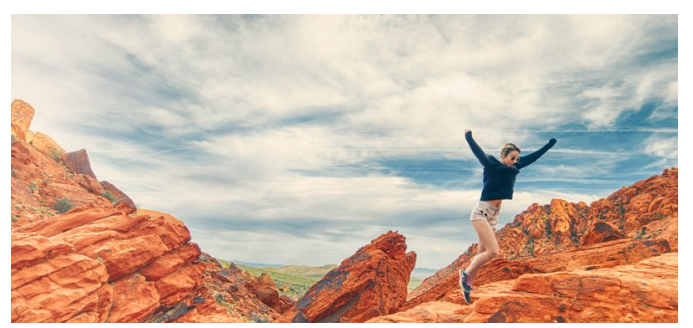

\section{The passion of the open scientist}

"A passion for knowledge, idle curiosity, altruistic concern with the benefit to humanity, and a host of other special motives have been attributed to the scientist"(Merton 1942).

"I didn't realize it at the time, but it was a turning point for me in my life. I had decided to stop chasing the money, and start chasing the passion. I was ready for the next chapter in my life"(Hsieh 2010).

John Hagel III has recently offered research suggesting that scientists are actually unavailable to be engaged as productive employees (something that university chancellors have long realized). And so, the "community engagement" efforts of college deans, learned societies and academic publishers will necessarily fail, and for a good reason. Perhaps for the best reason.

Hagel notes that "engaged" employees or customers are those who report they are happy with/in their current job, or with the current product/service. They have achieved a static form of satisfaction. From this disposition they can be relied upon to work harder or to buy more. Hagel's argument is supported by a long-term research project he helped lead at the Deloitte Center for the Edge. See: Exploring Passion (Accessed June 8, 2019). See also: Unlocking the Passion of the Explorer (Accessed June 8, 2019).

After decades of thousands of corporate engagement programs across the US, only about $30 \%$ of employees (in their survey) self-report as engaged (op cit). The bulk of the remainder are unhappy for a variety of reasons. Among the serially unengaged are those who come to work or to the marketplace following their own passions. 
Hagel is most interested in three passionate dispositions that he claims can add a lot of value to a company in today's emergent economy, well above the return on any engagement program. Combined, these dispositions form what he calls "the passion of the explorer." This description can be extended to include "knowledge explorers": scientists (including librarians and data scientists).

Hagel (op cit) writes:

"This form of passion has three components:

A long-term commitment to achieving an increasing impact in a domain; A questing disposition that creates excitement when confronted with an unexpected challenge;

A connecting disposition that motivates the individual to systematically seek out others who can help them to get to a better answer faster when confronted with an unexpected challenge."

That's a powerful combination. People with the passion of the explorer are never satisfied or happy with what they have accomplished. What excites them is the next challenge on the horizon-it's an opportunity to achieve more of their potential and take their impact in the domain to the next level. They are constantly seeking out those challenges and connecting with anyone who can help them address the challenge.

\section{Six rules about passion in the workplace:}

Passion is powerful, and it can shape teams interactions in a undesirable manner without some guidance.

1) Your passion is not a license to be the asshole in your group. You can use your passion to be an asshole with yourself only (well, and with your own research methods). (Sutton 2006; Accessed June 4, 2019).

2) Passion is also not more important than intellectual humility. (Resnick 2019; Accessed June 4, 2019). You can be passionate about learning something new, but don't let this bleed over into being passionate about what you think you already know. That's when you stop learning. Besides, intellectually humble people "possess more knowledge" (KrumreiMancuso et al. 2019).

3) Actually passionate people don't say they are. They act passionately, but don't profess this (Schmidt and Rosenberg 2019). They will confess, however, under light interrogation, or a few beers.

4) Having a colleague who is passionate about their work is no excuse to offload extra work on them, simply because they might do this without complaint. Do not abuse passionate 
workers in this way. They will leave and be passionate in another organization (Kim 2019; Accessed June 9, 2019).

5) During discussions about values and visions, passion needs to give way to humble listening (Schein 2013). Everyone has a voice, each person has a say. You get your time to contribute, but no more. Become passionate about the values the whole team decides are core values.

6) For those who are waiting to "find your passion": Don't wait for it to show up, develop your passion a little more each day. "Urging people to find their passion may lead them to put all their eggs in one basket but then to drop that basket when it becomes difficult to carry" (O'Keefe et al 2018). If there really is nothing in your job that you can connect to with passion, then you might want to ask yourself where else might you belong.

\section{How to engage the already engaged}

Passionate employees (in Hagel's sense) are predictably unhappy with the status quo. Of course, an original meaning of "passion" is "to suffer." They are necessarily immune from becoming engaged, and, one might guess, reactive to attempts made to engage them. In a 20th Century mode, these are not ideal employees. But the Deloitte study claims that these are precisely the type of employee needed for a 21st Century corporation.

In the academy, these are the scientists, intellectuals who are passionate about their research, who are eager to teach others, and who are resource-aggressive for any new knowledge they can acquire. Attempts to improve their "engagement" in some form of community will find them refractory in the extreme. Gamification will leave them merely irritated. Emails to them will be deleted unread.

The only community these scientists will really join, and then with some hesitation, are those they own and manage by themselves for their own purposes. They are happiest when they can be connected to others who share their specific objects of study, and even there, their discussions point to unknowns and pain-points in the research process.

How then can these passionate scientists be encouraged to connect, to coordinate their efforts, and collaborate online? What skills and knowledge do academic societies and universities need to acquire to move beyond engagement in order to unleash the collective intelligence of these scientists?

"What many people do not realize is that motivation by fear is indeed highly effective-effective at creating the illusion that goals are being achieved. It is not effective in ensuring that people bring the creativity, good process, and passion needed to accomplish challenging goals in knowledge-intensive workplaces" (Edmonson 2019). 
Open science offers a new level of support for the passion of the knowledge explorer. The best environment for this support will be new clubs, research/teaching collectives, and open collaborative networks where small groups of shared-vision/problem-minded scientists work together instead of alone.

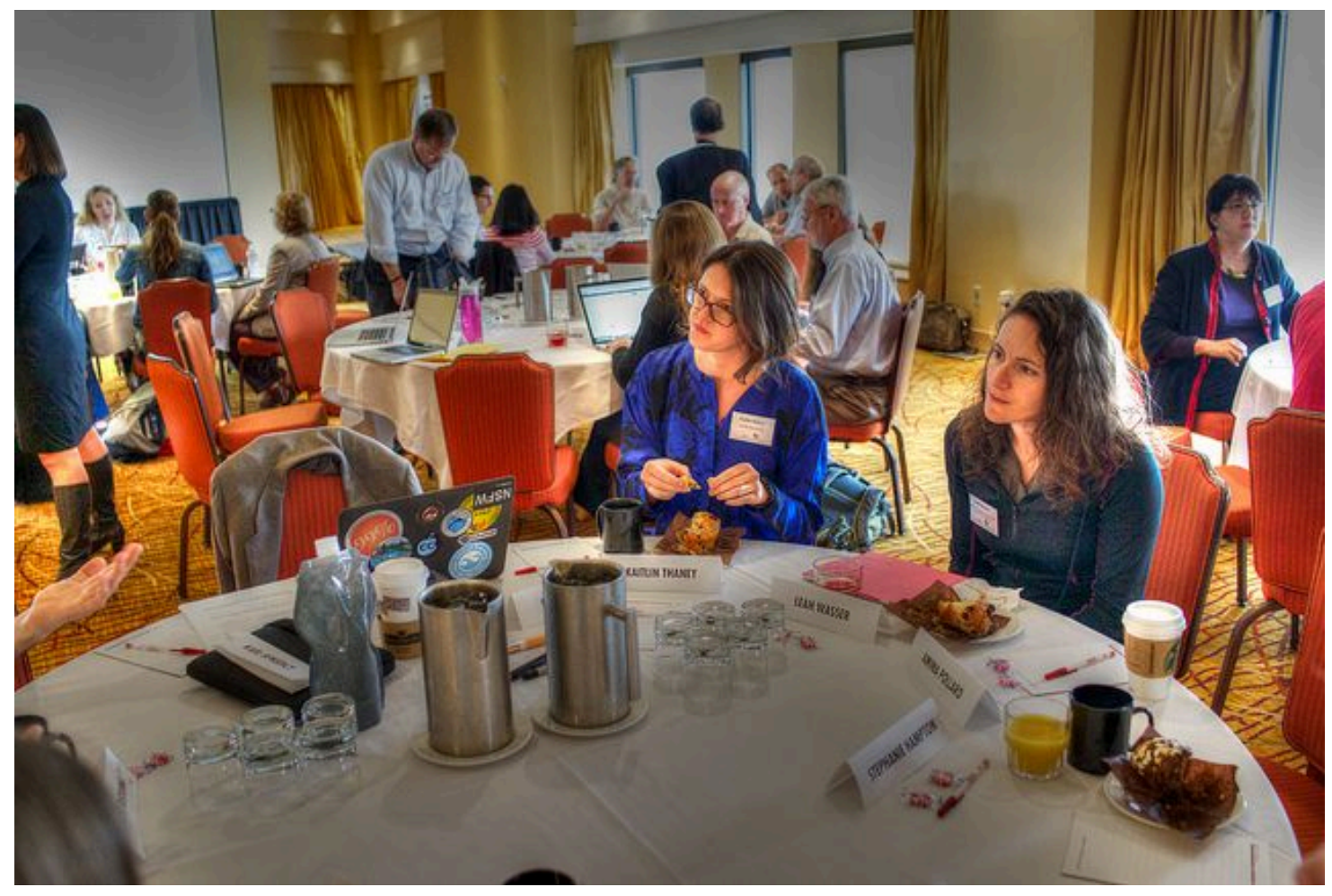

\section{What's the final word on Open Science?}

There is no final word here. We are still into beginnings. What is clear is that open science will help science expand and collaborate planet-wide. These new capabilities will leverage local public investments by the state and society, rewarding these with new value-added capabilities. Every locale will get more than it gives in this transaction.

Getting to open science will require inserting cultural practices into existing organizations and bootstrapping new ones with open science values and shared governance. You are a culture change agent. But you don't need to change the world, just the corner of it where you work and live. And you are not alone. The best way to start is to get into conversation with others. Real conversation, where you listen more than talk. 
Perhaps this Handbook has given you a few things to bring into that conversation. If so, then it's done its job. Pass it on to others, and make a ruckus.

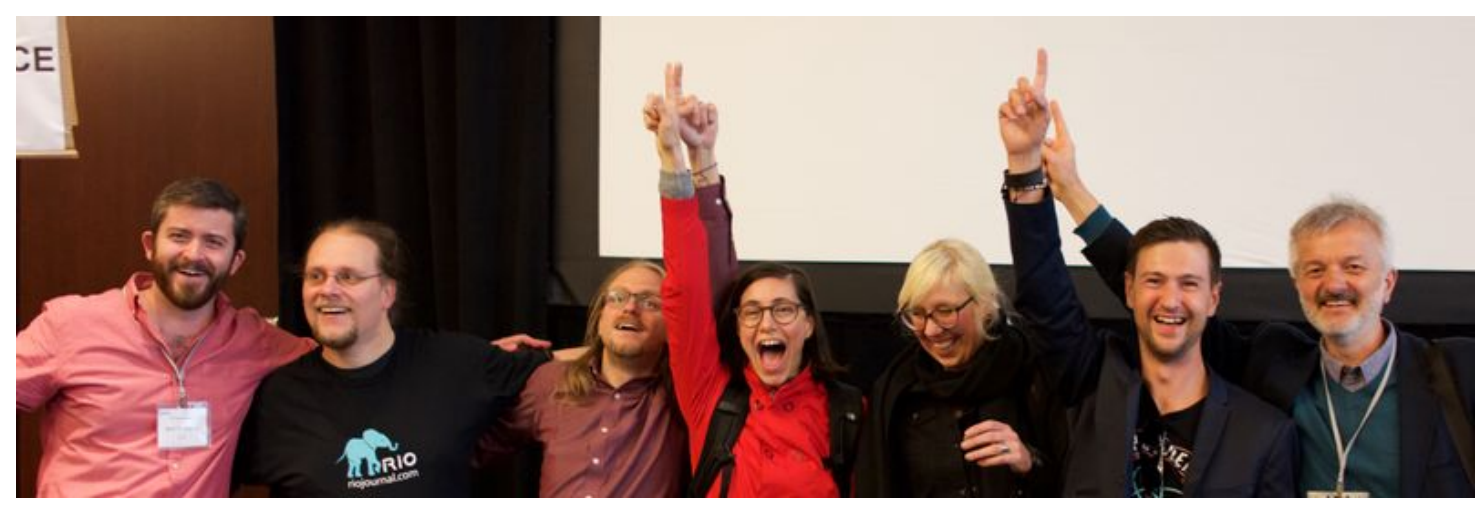




\section{Bibliography}

\section{This Bibliography is also sharable on Zotero here.}

Abambres, Miguel, Tony Salloom, and Nejra Beganovic. 2019. "Bye Bye Peer-Reviewed Publishing." Preprint. Open Science Framework. https://doi.org/10.31219/osf.io/4ezvf.

Abbott, Mark R. 2009. "A New Path for Science?" In The Fourth Paradigm: Data-Intensive Scientific Discovery, 111-16. Redmond, Washington: Microsoft Research.

Aleksic, Jelena, Adrian Alexa, Teresa K Attwood, Neil Chue Hong, Martin Dahlö, Robert Davey, Holger Dinkel, et al. 2015. "An Open Science Peer Review Oath." F1000Research 3 (January): 271. https://doi.org/10.12688/f1000research.5686.2.

Alon, Uri. 2010. “How to Build a Motivated Research Group.” Molecular Cell 37 (2): 151-52. https://doi.org/10.1016/j.molcel.2010.01.011.

Anderson, Benedict. 1983. Imagined Communities: Reflections on the Origin and Spread of Nationalism. London: Verso.

Anderson, Melissa S, Emily A. Ronning, Raymond De Vries, and Brian C. Martinson. 2010. "Extending the Mertonian Norms: Scientists' Subscription to Norms of Research." The Journal of Higher Education 81 (3): 366-93. https://doi.org/10.1353/jhe.0.0095.

Anderson, Melissa S., Brian C. Martinson, and Raymond De Vries. 2007. "Normative Dissonance in Science: Results from a National Survey of U.S. Scientists." Journal of Empirical Research on Human Research Ethics 2 (4): 3-14. https://doi.org/10.1525/ jer.2007.2.4.3.

Appadurai, Arjun. 1996. Modernity al Large: Cultural Dimensions of Globalization. Vol. 1. U of Minnesota Press.

Arbinger Institute. 2010. Leadership and Self-Deception: Getting out of the Box. BerrettKoehler Publishers.

Argyris, C., and D. Schön. 1978. Organizational Learning: A Theory of Action Perspective. Reading, Mass: Addison Wesley.

Argyris, Chris. 1982. “The Executive Mind and Double-Loop Learning." Organizational Dynamics 11 (2): 5-22.

——_. 1986. “Skilled Incompetence." Harvard Business Review 64 (5): 74-79. 
- - 2000. Flawed Advice and the Management Trap: How Managers Can Know When They're Getting Good Advice and When They're Not. Oxford: New York : Oxford University Press.

- - - 2004. "Double-Loop Learning and Organizational Change: Facilitating Transformational Change." Dynamics of Organizational Change and Learning, 389-402.

Ashford, Elizabeth, and Tim Mulgan. 2018. "Contractualism." In The Stanford Encyclopedia of Philosophy, edited by Edward N. Zalta, Summer 2018. Metaphysics Research Lab, Stanford University. https://plato.stanford.edu/archives/sum2018/entries/ contractualism/.

Ashkanasy, N.M., C.P.M. Wilderom, and M.F. Peterson, eds. 2000. Hand Book of Organizational Culture and Climate. Thousand Oaks. CA: Sage.

Association, American Library, and others. 2012. "Diversity Standards: Cultural Competency for Academic Libraries." Retrieved April 1: 2018.

Bacon, Jono. 2009. The Art of Community: Building the New Age of Participation. Sebastapol:O'Reilly. Available At. http://www.artofcommunityonline.org/downloads/ jonobacon-theartofcommunity-1ed.pdf.

Badiou, Alain, and Fabien Tarby. 2013. Philosophy and the Event. Translated by Louise Burchill. Cambridge: Polity.

Bailey, Michael, and Des Freedman, eds. 2011. The Assault on Universities: A Manifesto for Resistance. London : New York, NY: Pluto Press ; Distributed in the United States by Palgrave Macmillan, a Division of St. Martin's Press.

Banker, Chloe C, and Mark R Leary. 2019. "Hypo-Egoic Nonentitlement as a Feature of Humility." Personality and Social Psychology Bulletin, 0146167219875144.

Barsade, Sigal G., and Olivia A. O'Neill. 2014. “What's Love Got to Do with It? A Longitudinal Study of the Culture of Companionate Love and Employee and Client Outcomes in a LongTerm Care Setting." Administrative Science Quarterly 59 (4): 551-98. https://doi.org/ 10.1177/0001839214538636.

Bartling, Sönke, and Sascha Friesike, eds. 2014. Opening Science: The Evolving Guide on How the Internet Is Changing Research, Collaboration and Scholarly Publishing. Heidelberg: Springer Open.

Battaly, Heather, ed. 2019. The Routledge Handbook of Virtue Epistemology. New York London: Routledge, Taylor \& Francis Group. 
Bauer, Johannes, and Michael Latzer, eds. 2016. Handbook on the Economics of the Internet. Cheltenham, UK ; Northampton, MA: Edward Elgar Publishing.

Bauwens, M. 2012. "Evolving Towards a Partner State in an Ethical Economy." Accessible at: $<$ <ttp://Realitysandwich.Com/142773/Evolving_partner_state_ethical_economy/>. Accessed.

Bayet, A. 1947. La morale de la science. Société des éditions rationalistes.

Benkler, Yochai. 2006. The Wealth of Networks: How Social Production Transforms Markets and Freedom. New Haven [Conn.]: Yale University Press.

_-_ 2011. The Penguin and the Leviathan: How Cooperation Triumphs over Self-Interest. Crown Business.

-_- 2013. "Commons and Growth: The Essential Role of Open Commons in Market Economies." The University of Chicago Law Review 80 (3): 1499-1555.

- - 2 2015. "The Idea of the Commons \& Future of Capitalism." Presented at the Creative Commons Global Summit Seoul, Korea, October 15, 2015. https://www.slideshare.net/ cckslide/the-idea-of-the-commons-future-of-capitalism-yochai-benkler.

_- 2016. "Peer Production and Cooperation." Handbook on the Economics of the Internet 91.

_- _ 2017. "Open Access and Information Commons." The Oxford Handbook of Law and Economics: Volume 2: Private and Commercial Law, 256.

Benkler, Yochai, and Helen Nissenbaum. 2006. "Commons-Based Peer Production and Virtue." Journal of Political Philosophy 14 (4): 394-419.

Bereiter, Carl, and Marlene Scardamalia. 2016. “'Good Moves' in Knowledge-Creating Dialogue." Open and Interdisciplinary Journal of Technology, Culture and Education 11 (2): 12-26.

Berg, Maggie, and Barbara Karolina Seeber. 2016. The Slow Professor: Challenging the Culture of Speed in the Academy. Toronto: University of Toronto Press.

Berger, Warren. 2014. A More Beautiful Question: The Power of Inquiry to Spark Breakthrough Ideas. Bloomsbury Publishing USA.

Bergstrom, Theodore C, Paul N Courant, R Preston McAfee, and Michael A Williams. 2014. "Evaluating Big Deal Journal Bundles." Proceedings of the National Academy of Sciences 111 (26): 9425-9430. 
Best, Steven. 1991. Postmodern Theory: Critical Interrogations. Macmillan International Higher Education.

Best, Steven, and Douglas Kellner. 1997. The Postmodern Turn. Guilford Press.

Bilder, Geoffrey, Jennifer Lin, and Cameron Neylon. 2015. "Principles for Open Scholarly Infrastructures." Science in the Open.

Binswanger, Mathias. 2014. "Excellence by Nonsense: The Competition for Publications in Modern Science." In Opening Science, edited by Sönke Bartling and Sascha Friesike, 49-72. Cham: Springer International Publishing. https://doi.org/10.1007/978-3-319-00026-8_3.

—_- 2015. "How Nonsense Became Excellence: Forcing Professors to Publish." In Incentives and Performance, edited by Isabell M. Welpe, Jutta Wollersheim, Stefanie Ringelhan, and Margit Osterloh, 19-32. Cham: Springer International Publishing. https:// doi.org/10.1007/978-3-319-09785-5_2.

Bissola, Rita, Barbara Imperatori, and Alfredo Biffi. 2017. "A Rhizomatic Learning Process to Create Collective Knowledge in Entrepreneurship Education: Open Innovation and Collaboration beyond Boundaries." Management Learning 48 (2): 206-26. https://doi.org/ $10.1177 / 1350507616672735$.

Björk, B.C. 2017. "Scholarly Journal Publishing in Transition-from Restricted to Open Access." Electronic Markets 2 (101-109).

Blanchard, A.L.and M.L.Markus. 2002. "Sense of Virtual Community - Maintaining the Experience of Belonging." In Proceedings of the 35th Hawaii International Conference on System Sciences.

Boik, J.C. 2016. “Optimality of Social Choice Systems: Complexity, Wisdom, and Wellbeing Centrality." Open Science Framework. December, 9.

Boland Jr, Richard J, and Ramkrishnan V Tenkasi. 1995. "Perspective Making and Perspective Taking in Communities of Knowing." Organization Science 6 (4): 350-372.

Bollier, David. 2017. "Re-Imagining Value: Insights from the Care Economy, Commons, Cyberspace and Nature." Berlin: Heinrich Böll Siftung.

Bollier, David, and Silke Helfrich. 2019. Free, Fair, and Alive: The Insurgent Power of the Commons.

Bourdieu, Pierre. 1990a. In Other Words: Essays towards a Reflexive Sociology. Stanford University Press. 
_- _ 1990b. The Logic of Practice. Stanford: Stanford University Press. [Originally Le Sens Practique].

Bowles, Samuel, and Sandra Polania-Reyes. 2012. "Economic Incentives and Social Preferences: Substitutes or Complements?" Journal of Economic Literature 50 (2): 368-425.

Boyle, J. 2007. “Mertonianism Unbound?: imagining free, decentralized access to most cultural and scientific material." In , edited by C. Hess and E. Ostrom. The MIT press.

- - 2008. The Public Domain: Enclosing the Commons of the Mind. New: Haven. Yale University Press.

Branch Jr, William T, and Gary A Mitchell. 2011. “Wisdom in Medicine.” Pharos Alpha Omega Alpha Honor Med Soc 74: 12-17.

Brook, Freeda, Dave Ellenwood, and Althea Eannace Lazzaro. 2015. "In Pursuit of Antiracist Social Justice: Denaturalizing Whiteness in the Academic Library." Library Trends 64 (2): 246-284.

Broß, Justis, Harold Sack, and and Christof Meinel. 2007. Encouraging Participation in Virtual Communities: The "IT-Summit-Blog" Case. ESociety. http://www.informatik.unijena.de/.

Brown, Brené. 2007. I Thought It Was Just Me: Women Reclaiming Power and Courage in a Culture of Shame. Gotham New York.

- - 2012. Daring Greatly: How the Courage to Be Vulnerable Transforms the Way We Live, Love, Parent, and Lead. 1st ed. New York, NY: Gotham Books.

Brown, J.S.and P.Duguid. 1991. “Organizational Knowledge and Communities of Practice.” Organization Science 2 (1): 40-57.

Brown, Stuart L. 2009. Play: How It Shapes the Brain, Opens the Imagination, and Invigorates the Soul. Penguin.

Buckheit, Jonathan B, and David L Donoho. 1995. "Wavelab and Reproducible Research.” In Wavelets and Statistics, 55-81. Springer.

Buckingham, Marcus, and Ashley Goodall. 2019. Nine Lies about Work: A Freethinking Leader's Guide to the Real World. Boston, Massachusetts: Harvard Business Review Press.

Bush, Vannevar. 1945. Science, the Endless Frontier: A Report to the President. US Govt. print. off. 
Butler, Brian, Lee Sproull, Sara Kiesler, and Robert Kraut. 2007. “Community Effort in Online Groups: Who Does the Work and Why?" In Leadership at a Distance: Research in Technologically-Supported Work. Suzanne, edited by P. Weisband. Psychology Press.

Cantrell, Deborah J, and Kenneth Sharpe. 2015. "Practicing Practical Wisdom." Mercer L. Rev. 67: 331.

Carlson, Stephanie M, and Rachel E White. 2013. "Executive Function, Pretend Play, and Imagination." The Oxford Handbook of the Development of Imagination, 161-174.

Caron, Bruce. 2017. “Serious Games and the Study of Society." https://doi.org/10.6084/ m9.figshare.3206233.v2.

Carse, James P. 1987. Finite and Infinite Games. Ballantine Books.

Casadevall, Arturo, and Ferric C Fang. 2012a. Reforming Science: Methodological and Cultural Reforms. Am Soc Microbiol.

___ 2012b. “Winner Takes All." Scientific American 307 (2): 13-17.

_—_. 2013. "Is the Nobel Prize Good for Science?" The FASEB Journal 27 (12): 46824690.

Caswell, Michelle, and Marika Cifor. 2016. "From Human Rights to Feminist Ethics: Radical Empathy in the Archives." Archivaria 81 (1): 23-43.

Chapman, Colin A., Júlio César Bicca-Marques, Sébastien Calvignac-Spencer, Pengfei Fan, Peter J. Fashing, Jan Gogarten, Songtao Guo, et al. 2019. “Games Academics Play and Their Consequences: How Authorship, $h$-Index and Journal Impact Factors Are Shaping the Future of Academia." Proceedings of the Royal Society B: Biological Sciences 286 (1916): 20192047. https://doi.org/10.1098/rspb.2019.2047.

Chen, Katherine K. 2009. Enabling Creative Chaos: The Organization Behind the Burning Man Event. Chicago: University of Chicago Press. Kindle Edition.

Chipuer, H.M., and G.M.H. Pretty. 1999. "A Review of the Sense of Community Index: Current Uses, Factor Structure, Reliability, and Further Development." Journal of Community Psychology 27 (6): 643-658.

Chomsky, N. 1966. Current Issues in Linguistic Theory. Vol. 38. Mouton.

Chopra, Vineet, Dana P Edelson, and Sanjay Saint. 2016. “Mentorship Malpractice.” Jama 315 (14): 1453-1454. 
Chu, Clara M. 1999. “Transformative Information Services: Uprooting Race Politics." In Presentation, Black Caucus of the American Library Association Conference, Las Vegas, 175204.

Clancy, Kathryn B. H., Robin G. Nelson, Julienne N. Rutherford, and Katie Hinde. 2014. "Survey of Academic Field Experiences (SAFE): Trainees Report Harassment and Assault." Edited by Coren Lee Apicella. PLoS ONE 9 (7): e102172. https://doi.org/10.1371/ journal.pone.0102172.

Clark, David H., and Stephen P. H. Clark. 2001. Newton's Tyranny: The Suppressed Scientific Discoveries of Stephen Gray and John Flamsteed. New York: W.H. Freeman and Co.

Clegg, S., and S. Rowland. 2010. "Kindness in pedagogical practice and academic life." British Journal of Sociology of Education 31 (6): 719-735.

Cloninger, C. Robert, and Ada H. Zohar. 2011. "Personality and the Perception of Health and Happiness." Journal of Affective Disorders 128 (1-2): 24-32. https://doi.org/10.1016/ j.jad.2010.06.012.

Cohen, A.P. 1985. The Symbolic Construction of Community. Chichester, Sussex: Ellis Horwood Ltd.

Cook, D.N., and John Seely Brown. 1999. "Bridging Epistemologies: The Generative Dance between Organizational Knowledge and Organizational Knowing." Organization Science 10 (4): 381-400. http://www.jstor.org/stable/2640362.

Cook, S.D.N., and D. Yanow. 1993. "Culture and organizational learning." Journal of Management Inquiry 2 (4): 373-390.

Copeland, Samantha. 2019. "On Serendipity in Science: Discovery at the Intersection of Chance and Wisdom." Synthese 196 (6): 2385-2406. https://doi.org/10.1007/ s11229-017-1544-3.

Cosley, Dan. 2005. Mining Social Theory to Build Member-Maintained Communities. AAAI.

Cosley, Dan, Dan Frankowski, Sara Kiesler, Loren Terveen, and John Riedl. 2005. “How Oversight Improves Member-Maintained Communities." In Proceedings of the CHI.

Crawford, S.E., and E. Ostrom. 1995. "A grammar of institutions." American Political Science Review 89 (3): 582-600.

Crick, Tom, Benjamin A Hall, and Samin Ishtiaq. 2015. "Reproducibility in Research: Systems, Infrastructure, Culture." ArXiv Preprint ArXiv:1503.02388. 
Csikszentmihalyi, Mihaly, and Isabella Selega Csikszentmihalyi. 1992. Optimal Experience: Psychological Studies of Flow in Consciousness. Cambridge university press.

Csikszentmihalyi, Mihalyi. 1991. Flow: The Psychology of Optimal Experience. New York: Harper Collins.

Curry, Stephen. 2018. "DORA, Plan S and the (Open) Future of Research Evaluation." Presented at the Science Europe General Assembly, Brussels, November 22.

Dasgupta, Partha, and Paul A David. 1994. "Toward a New Economics of Science." Research Policy 23 (5): 487-521.

Dastur, F. 2017. "How to Open an Academic Department." In , edited by R.S. Jhangiani and R. Biswas-Diener, 163-178. London: Ubiquity Press. https://doi.

David, Paul A. 1998. "Communication Norms and the Collective Cognitive Performance of 'Invisible Colleges."' In Creation and Transfer of Knowledge, 115-163. Springer.

Davies, Bronwyn. 2005. "The (Im)Possibility of Intellectual Work in Neoliberal Regimes." Discourse: Studies in the Cultural Politics of Education 26 (1): 1-14. https://doi.org/ 10.1080/01596300500039310.

Deal, T.E., and A.A. Kennedy. 1999. The New Corporate Cultures. New York: Perseus.

Deal, Terrence, and Allan Kennedy. 1982. "Corporate Cultures." The Rites and Rituals of Corporate Life. London, Pinguin Books.

Debord, Guy. 2010. The Society of the Spectacle. Reprint. Detroit, Mich: Black \& Red [u.a.].

Deutsch, David. 2011. The Beginning of Infinity: Explanations That Transform the World. Penguin UK.

Dintersmith, Ted. 2018. What School Could Be: Insights and Inspiration from Teachers across America. Princeton University Press.

DiPrete, Thomas A., and Gregory M. Eirich. 2006. "Cumulative Advantage as a Mechanism for Inequality: A Review of Theoretical and Empirical Developments." Annual Review of Sociology 32 (1): 271-97. https://doi.org/10.1146/annurev.soc.32.061604.123127.

Diver, Colin. 2005. “Is There Life after Rankings?” Atlantic Monthly 296 (4): 136.

Doctorow, Cory. 2014. Information Doesn't Want to Be Free: Laws for the Internet Age. McSweeney's. 
Donskis, Leonidas, ed. 2019. Academia in Crisis: Dystopic Optimism and Postalgic Realism in University Life. Value Inquiry Book Series, volume 335. Leiden ; Boston: Brill-Rodopi.

Douglas, M. 1986. How Institutions Think. Syracuse, NY: Syracuse: University Press.

Dowd, Karen O., and David M. Kaplan. 2005. "The Career Life of Academics: Boundaried or Boundaryless?" Human Relations 58 (6): 699-721. https://doi.org/ $10.1177 / 0018726705057156$.

Drucker, Peter Ferdinand. 2001. The Essential Drucker. Oxford: Butterworth-Heinemann.

Drutman, Lee. 2012. "How the NSF Allocates Billions of Federal Dollars to Top Universities." Sunlight Foundation Blog.

Duckles, Beth M., Mark A. Hager, and and Joseph Galaskiewicz. 2005. "How Nonprofits Close: Using Narratives to Study Organizational Processes." In Qualitative Organizational Research: Best Papers from the Davis Conference on Qualitative Research, edited by Kimberly D. Elsbach, 169-203. Greenwich, CT: Information Age Publishing.

Dudley, Drew. 2018. This Is Day One: A Practical Guide to Leadership That Matters. First edition. New York: Hachette Books.

Dunwoody, Ann, and Tomago Collins. 2015. A Higher Standard: Leadership Strategies from America's First Female Four-Star General. First Da Dapo Press edition. Boston, MA: Da Capo Lifelong, a member of the Perseus Books Group.

Edmondson, Amy C. 2019. The Fearless Organization: Creating Psychological Safety in the Workplace for Learning, Innovation, and Growth. Hoboken, New Jersey: John Wiley \& Sons, Inc.

Edwards, Marc A., and Siddhartha Roy. 2017. "Academic Research in the 21st Century: Maintaining Scientific Integrity in a Climate of Perverse Incentives and Hypercompetition." Environmental Engineering Science 34 (1): 51-61. https://doi.org/10.1089/ees.2016.0223.

Eghbal, N. 2018. Roads and Bridges: The Unseen Labor Behind Our Digital Infrastructure. White Paper. Ford Foundation.

Einstein, Albert. 1960. Ideas and Opinions. Crown Trade Paperbacks New York.

Eisfeld-Reschke, Jörg, Ulrich Herb, and Karsten Wenzlaff. 2014. "Research Funding in Open Science." In Opening Science, edited by Sönke Bartling and Sascha Friesike, 237-53. Cham: Springer International Publishing. https://doi.org/10.1007/978-3-319-00026-8_16.

Europäische Kommission, ed. 2016. Open Innovation, Open Science, Open to the World: A Vision for Europe. Luxembourg: Publications Office of the European Union. 
Fang, Ferric C, and Arturo Casadevall. 2016. Research Funding: The Case for a Modified Lottery. Am Soc Microbiol.

Feinberg, M., J.T. Cheng, and Willer, R. 2012. “Gossip as an Effective and Low-Cost Form of Punishment." Behavioral and Brain Sciences 35 (1): 25-25.

Feinberg, M., R. Willer, J. Stellar, and D. Keltner. 2012. “The Virtues of Gossip: Reputational Information Sharing as Prosocial Behavior." Journal of Personality and Social Psychology 102 (5): 1015.

Feist, Gregory J. 2006. The Psychology of Science and the Origins of the Scientific Mind. New Haven: Yale University Press.

Feynman, Richard Phillips. 2006. QED: The Strange Theory of Light and Matter. Princeton University Press.

Feynman, R.P., J. Robbins, H. Sturman, and A. Löhnberg,. 2005. The Pleasure of Finding Things Out. Nieuw Amsterdam.

Fine, Michelle, and Susan Merle Gordon. 1991. "Effacing the Center and the Margins: Life at the Intersection of Psychology and Feminism." Feminism \& Psychology 1 (1): 19-27. https:// doi.org/10.1177/0959353591011002.

Finholt, Thomas A., Lee Sproull, and Sara Keisler. 2002. Outsiders on the Inside: Sharing Know-How across Time and Space. Edited by Sara Keisler and Pamala J. Hinds. Distributed Work. Boston: MIT Press.

Fire, Michael, and Carlos Guestrin. 2019. "Over-Optimization of Academic Publishing Metrics: Observing Goodhart's Law in Action." GigaScience 8 (6): giz053. https://doi.org/ 10.1093/gigascience/giz053.

Firestein, Stuart. 2012. Ignorance: How It Drives Science. New York: Oxford University Press.

Fitzpatrick, Kathleen. 2019. Generous Thinking: A Radical Approach to Saving the University. Baltimore: Johns Hopkins University Press.

Foray, Dominique. 2004. Economics of Knowledge. MIT press.

Foray, Dominique, and Francesco Lissoni. 2010. "University Research and Public-Private Interaction." In Handbook of the Economics of Innovation, 1:275-314. Elsevier. https:// doi.org/10.1016/S0169-7218(10)01006-3.

Foucault, Michel, Arnold I Davidson, and Graham Burchell. 2008. The Birth of Biopolitics: Lectures at the Collège de France, 1978-1979. Springer. 
Frankfurt, Harry G. 2009. On Bullshit. Princeton University Press.

Freistetter, F. 2018. Isaac Newton: The Asshole Who Reinvented the Universe. First American hardcover edition in English. Amherst, New York: Prometheus Books.

Frey, B.S. 2003. "Publishing as Prostitution?-Choosing between One's Own Ideas and Academic Success.” Public Choice 116 (1-2): 205-223.

Frith, Uta. 2019. "Fast Lane to Slow Science." Trends in Cognitive Sciences, November, S1364661319302426. https://doi.org/10.1016/j.tics.2019.10.007.

Fukuyama, Francis. 1995. Trust: The Social Virtues and the Creation of Prosperity. Vol. 99. Free press New York.

Fyfe, Aileen, Kelly Coate, Stephen Curry, Stuart Lawson, Noah Moxham, and Camilla Mørk Røstvik. 2017. "Untangling Academic Publishing: A History of the Relationship between Commercial Interests, Academic Prestige and the Circulation of Research." Zenodo. https:// doi.org/10.5281/zenodo.546100.

Gagliardi, P., ed. 1990. Symbols and Artifacts: Views of the Corporate Landscape. New York: Walter de Gruyter.

Galegher, Jolene, Lee Sproull, and Sara Kiesler. 1998. "Legitimacy, authority and community in electronic support groups." Written Communication 15: 493-530.

Garcia, Danilo, and Patricia Rosenberg. 2016. “The Dark Cube: Dark and Light Character Profiles." PeerJ 4 (February): e1675. https://doi.org/10.7717/peerj.1675.

Gardner, Rita. 2013. “Open Access and Learned Societies." Vincent and Wickham (2013), 1329.

Gay, Geneva. 2010. Culturally Responsive Teaching: Theory, Research, and Practice. Teachers College Press.

Geertz, C. 1973. The Interpretation of Cultures. New York: Basic Books.

Geertz, Clifford. 1973. Thick Description: Toward an Interpretive Theory of Culture. in Clifford Geertz, The Interpretation of Cultures.

Giamatti, A.Bartlett. 1989. Take Time for Paradise: Americans and Their Games. New York: Summit Books.

Giddens, Anthony. 1991. Modernity and Self-Identity: Self and Society in the Late Modern Age. Stanford university press. 
_-_ 1994. Beyond Left and Right: The Future of Radical Politics. Stanford: Stanford University Press.

Ginsberg, Benjamin. 2011. The Fall of the Faculty. Oxford University Press.

Gleick, James. 2004. Isaac Newton. New York; Westminster: Vintage Imprint ; Knopf Doubleday Publishing Group ; Random House, Incorporated Distributor. http:// site.ebrary.com/id/10063736.

——_ 2011. The Information: A History, a Theory, a Flood. 1st ed. New York: Pantheon Books.

Glück, Judith. 2017. “... and the Wisdom to Know the Difference: Scholarly Success From a Wisdom Perspective." Perspectives on Psychological Science 12 (6): 1148-52. https:// doi.org/10.1177/1745691617727528.

__— 2018a. "Measuring Wisdom: Existing Approaches, Continuing Challenges, and New Developments." The Journals of Gerontology: Series B 73 (8): 1393-1403. https://doi.org/ 10.1093/geronb/gbx140.

- - 2 2018b. "New Developments in Psychological Wisdom Research: A Growing Field of Increasing Importance." The Journals of Gerontology: Series B 73 (8): 1335-38. https:// doi.org/10.1093/geronb/gby102.

Glück, Judith, and Susan Bluck. 2013. "The MORE Life Experience Model: A Theory of the Development of Personal Wisdom." In The Scientific Study of Personal Wisdom, 75-97. Springer.

Godin, Seth. 2012. Stop Stealing Dreams:(What Is School For?).

Goffman, E. 1959. “The Presentation of Self in Everyday Life." In . New York: Doubleday.

_-_ 1967. Interaction Ritual. Hawthorne, NY: Aldine.

Goldman, Ron, and Richard P. Gabriel. 2005. Innovation Happens Elsewhere: Open Source as Business Strategy. Morgan Kaufmann.

Gopnik, A. 2012. "Scientific Thinking in Young Children: Theoretical Advances, Empirical Research, and Policy Implications.” Science 337 (6102): 1623-27. https://doi.org/10.1126/ science.1223416.

Graeber, David. 2001. Toward an Anthropological Theory of Value: The False Coin of Our Own Dreams. New York: Palgrave.

—__ 2011. Debt: The First 5,000 Years. Brooklyn, N.Y: Melville House. 
-_- 2014. "Anthropology and the Rise of the Professional-Managerial Class." $H A U$ : Journal of Ethnographic Theory 4 (3): 73-88. https://doi.org/10.14318/hau4.3.007.

——_. 2019. Bullshit Jobs: A Theory.

Grahe, Jon E, Kelly Cuccolo, Dana C Leighton, and Leslie D Cramblet Alvarez. 2020. “Open Science Promotes Diverse, Just, and Sustainable Research and Educational Outcomes." Psychology Learning \& Teaching 19 (1): 5-20. https://doi.org/ $10.1177 / 1475725719869164$.

Granovetter, M.S. 1977. “The Strength of Weak Ties.” In Social Networks, 347-367.

_-_ 1983. The Strength of Weak Ties: A Network Theory Revisited. Sociological Theory.

Grant, Adam M. 2013. Give and Take: A Revolutionary Approach to Success. New York, N.Y: Viking.

Grant, Sheryl. 2014. What Counts as Learning: Open Digital Badges for New Opportunities. Cork: BookBaby.

Greenhalgh, Trisha, Mustafa F. Ozbilgin, Barbara Prainsack, and Sara Shaw. 2019. "Moral Entrepreneurship, the Power-knowledge Nexus, and the Cochrane 'Crisis.'” Journal of Evaluation in Clinical Practice, March. https://doi.org/10.1111/jep.13124.

Grimm, Stephen R. 2015. “Wisdom.” Australasian Journal of Philosophy 93 (1): 139-154.

Gross, Kevin, and Carl T. Bergstrom. 2019. “Contest Models Highlight Inherent Inefficiencies of Scientific Funding Competitions." Edited by John PA Ioannidis. PLOS Biology 17 (1): e3000065. https://doi.org/10.1371/journal.pbio.3000065.

Grossmann, Alexander, and Björn Brembs. 2019. "Assessing the Size of the Affordability Problem in Scholarly Publishing." PeerJ Preprints 7 (June): e27809v1. https://doi.org/ 10.7287/peerj.preprints.27809v1.

Grossmann, Igor, Tanja M. Gerlach, and Jaap J. A. Denissen. 2016. "Wise Reasoning in the Face of Everyday Life Challenges." Social Psychological and Personality Science 7 (7): 61122. https://doi.org/10.1177/1948550616652206.

Grossmann, Igor, Jinkyung Na, Michael EW Varnum, Shinobu Kitayama, and Richard E Nisbett. 2013. "A Route to Well-Being: Intelligence versus Wise Reasoning." Journal of Experimental Psychology: General 142 (3): 944.

Hagel, John, and John Seely Brown. 2008. "Creation Nets: Harnessing The Potential Of Open Innovation." Journal of Service Science 1 (2): 27-40. 
Hagel, John, John Seely Brown, and Lang Davison. 2012. The Power of Pull: How Small Moves, Smartly Made, Can Set Big Things in Motion. Basic Books.

Hagstrom, Warren 0. 1965. The Scientific Community. New York: basic books.

Halberstam, Judith. 2011. The Queer Art of Failure. Durham: Duke University Press.

Hallward, Peter, ed. 2004. Think Again: Alain Badiou and the Future of Philosophy. London ; New York: Continuum.

Harari, Y.N. 2014. Sapiens: A Brief History of Humankind. Random House.

Hargrave-Thomas, Emily. 2012. "Serendipity in Anticancer Drug Discovery." World Journal of Clinical Oncology 3 (1): 1. https://doi.org/10.5306/wjco.v3.i1.1.

Harré, Niki, Barbara M Grant, Kirsten Locke, Sean Sturm, and others. 2017. “The University as an Infinite Game." Australian Universities' Review, The 59 (2): 5.

Hartley, John, Jason Potts, Lucy Montgomery, Ellie Rennie, and Cameron Neylon. 2019. “Do We Need to Move from Communication Technology to User Community? A New Economic Model of the Journal as a Club." Learned Publishing 32 (1): 27-35. https://doi.org/10.1002/ leap.1228.

Harvey, David. 1989. The Condition of Postmodernity. Vol. 14. Blackwell Oxford.

Hatch, M.J. 1990. "The Symbolics of Office Design." In , edited by P. Gagliardi. New York: Walter de Gruyter.

Hazelkorn, Ellen. 2019. "University Rankings: There Is Room for Error and 'Malpractice."” March 2019. https://doi.org/10.5281/zenodo.2592196.

Headlee, Celeste. 2018. We Need To Talk: How To Have Conversations That Matter. Harpercollins. http://www.vlebooks.com/vleweb/product/openreader? id=none\&isbn=9780062669025.

Heesen, Remco, and Jan-Willem Romeijn. 2019. "Epistemic Diversity and Editor Decisions: A Statistical Matthew Effect." Philosophers' Imprint 19 (39): 1-20.

Helfrich, Silke, and David Bollier. 2019. Free, Fair, and Alive: The Insurgent Power of the Commons.

Heller, M. A., and Rebecca S. Eisenberg. 1998. "Can Patents Deter Innovation? The Anticommons in Biomedical Research." Science 280 (5364): 698-701. https://doi.org/ 10.1126/science.280.5364.698. 
Henderson, Linda, Eileen Honan, and Sarah Loch. 2016. "The Production of the Academicwritingmachine." Reconceptualizing Educational Research Methodology 7 (2). https://doi.org/10.7577/rerm.1838.

Hess, Charlotte, and Elinor Ostrom, eds. 2009. Understanding Knowledge as a Commons. From Theory to Practice. Cambridge MA: The MIT Press.

Hesselbein, Frances. 1998. The Community of the Future. Edited by Marshall Goldsmith, Richard Bechard, and Richard F. Schubert. San Francisco: Josey-Bass.

Hey, Anthony J. G., ed. 2009. The Fourth Paradigm: Data-Intensive Scientific Discovery. Redmond, Washington: Microsoft Research.

Hey, V. 2004. "Perverse Pleasures-Identity Work and the Paradoxes of Greedy Institutions." Journal of International Women's Studies 5 (3): 33-43.

Hiatt, L.R. 1997. Scholar and Sceptic: Australian Aboriginal Studies in Honour of LR Hiatt. Aboriginal Studies Press.

Hildreth, Paul, and Chris Kimble. 2004. Knowledge Networks: Innovation through Communities of Practice. London: Hershey: Idea Group Inc.

Hirsch, Jorge E. 2005. "An Index to Quantify an Individual's Scientific Research Output." Proceedings of the National Academy of Sciences 102 (46): 16569-16572.

Hodson, Gordon, Angela Book, Beth A. Visser, Anthony A. Volk, Michael C. Ashton, and Kibeom Lee. 2018. "Is the Dark Triad Common Factor Distinct from Low HonestyHumility?" Journal of Research in Personality 73 (April): 123-29. https://doi.org/10.1016/ j.jrp.2017.11.012.

Hodson, Simon, Sarah Jones, Sandra Collins, Françoise Genova, Natalie Harrower, Leif Laaksonen, Daniel Mietchen, Rūta Petrauskaité, and Peter Wittenburg. 2018. “Turning FAIR Data into Reality: Interim Report from the European Commission Expert Group on FAIR Data," June. https://doi.org/10.5281/ZENODO.1285272.

Hoeschele, Wolfgang. 2010. The Economics of Abundance: A Political Economy of Freedom, Equity, and Sustainability. Gower Green Economics and Sustainable Growth Series. Farnham ; Burlington, VT: Gower.

Hofstede, G. 2001. Culture's consequences. 2nd ed.). Thousand, (1st ed. Oaks, CA: Sage.

Holbrook, J Britt. 2019. “Open Science, Open Access, and the Democratization of Knowledge." Issues in Science and Technology 35 (3): 26-28. 
Holcombe, Alex. 2019. "Farewell Authors, Hello Contributors." Nature 571 (7764): 147-147. https://doi.org/10.1038/d41586-019-02084-8.

Homans, G. 1950. The Human Group. New York: Harcourt Brace Jovanovich.

Honan, Eileen. 2014. "Disrupting the Habit of Interviewing." Reconceptualizing Educational Research Methodology 5 (1). https://doi.org/10.7577/rerm.929.

Honma, Todd. 2005. “Trippin'over the Color Line: The Invisibility of Race in Library and Information Studies." InterActions: UCLA Journal of Education and Information Studies 1 (2).

Horrobin, David F. 1996. "Peer Review of Grant Applications: A Harbinger for Mediocrity in Clinical Research?" The Lancet 348 (9037): 1293-1295.

Hsieh, Tony. 2010. Delivering Happiness: A Path to Profits, Passion, and Purpose. Hachette Book Group. Kindle Edition.

Hyde, Lewis. 2009. The Gift: Creativity and the Artist in the Modern World. Vintage.

Ito, Joi, and Jeff Howe. 2016. Whiplash: How to Survive Our Faster Future. Grand Central Publishing.

James, A. 2012. Fairness in Practice: A Social Contract for a Global Economy. Oxford University Press.

Jenks, Chris. 1993. Culture. London And. New York: Routledge.

Jeppesen, Lars Bo, and Karim R Lakhani. 2010. "Marginality and Problem-Solving Effectiveness in Broadcast Search." Organization Science 21 (5): 1016-1033.

Jeste, Dilip V., Ellen E. Lee, Charles Cassidy, Rachel Caspari, Pascal Gagneux, Danielle Glorioso, Bruce L. Miller, et al. 2019. "The New Science of Practical Wisdom." Perspectives in Biology and Medicine 62 (2): 216-36. https://doi.org/10.1353/pbm.2019.0011.

Jhangiani, Rajiv S., and Robert Biswas-Diener, eds. 2017. Open: The Philosophy and Practices That Are Revolutionizing Education and Science. Ubiquity Press. http://www.oapen.org/ download?type=document $\&$ docid $=627025$.

John, Leslie K., George Loewenstein, and Drazen Prelec. 2012. "Measuring the Prevalence of Questionable Research Practices With Incentives for Truth Telling." Psychological Science 23 (5): 524-32. https://doi.org/10.1177/0956797611430953.

Johnson, S. 2011. Where Good Ideas Come from: The Seven Patterns of Innovation. Penguin UK. 
Jones, Daniel Nelson, and Aurelio Jose Figueredo. 2013. "The Core of Darkness: Uncovering the Heart of the Dark Triad: The Core of Darkness." European Journal of Personality 27 (6): 521-31. https://doi.org/10.1002/per.1893.

Jones, M.O., M.D. Moore, and R.C. Snyder, eds. 1988. Inside organizations. Thousand Oaks. CA: Sage.

Joseph, Miranda. 2002. Against the Romance of Community. Minneapolis: University of Minnesota Press.

- - . 2017. “Community, Collectivity, Affinities." In A Companion to Critical and Cultural Theory, edited by Imre Szeman, Sarah Blacker, and Justin Sully, 205-21. Chichester, UK: John Wiley \& Sons, Ltd. https://doi.org/10.1002/9781118472262.ch12.

Juarrero, Alicia. 1999. Dynamics in Action. Cambridge, Ma: MIT Press.

Kahneman, Daniel. 2013. Thinking, Fast and Slow. 1st pbk. ed. New York: Farrar, Straus and Giroux.

Kalanithi, Paul, and A. Verghese. 2016. When Breath Becomes Air. First edition. New York: Random House.

Kaldjian, L. C. 2010. "Teaching Practical Wisdom in Medicine through Clinical Judgement, Goals of Care, and Ethical Reasoning." Journal of Medical Ethics 36 (9): 558-62. https:// doi.org/10.1136/jme.2009.035295.

Kao, John. 2002. "Reinventing Innovation: A Perspective from The Idea Factory." In Frances Hesselbein; Marshall Goldsmith; Iain Somerville. Leading for Innovation: And Organizing For Results. Kindle Edition. Originally Published By. Josey-Bass: New York.

Katz, Daniel S., Gabrielle Allen, Lorena A. Barba, Devin R. Berg, Holly Bik, Carl Boettiger, Christine L. Borgman, et al. 2018. "The Principles of Tomorrow's University." F1000Research 7 (December): 1926. https://doi.org/10.12688/f1000research.17425.1.

Kauffman, Stuart. 2014. At Home in the Universe The Search for the Laws of Self-Organization and Complexity. Cary: Oxford University Press, USA.

Kauffman, Stuart A. 2000. Investigations. Oxford University Press.

Kaufman, Scott Barry, David Bryce Yaden, Elizabeth Hyde, and Eli Tsukayama. 2019. "The Light vs. Dark Triad of Personality: Contrasting Two Very Different Profiles of Human Nature." Frontiers in Psychology 10: 467. https://doi.org/10.3389/fpsyg.2019.00467.

Kawasaki, Guy. 2012. Enchantment: The Art of Changing Hearts, Minds and Actions. Penguin UK. 
Keating, Brian. 2018. Losing the Nobel Prize: A Story of Cosmology, Ambition, and the Perils of Science's Highest Honor. WW Norton \& Company.

Keltner, Dacher, and Jonathan Haidt. 2003. "Approaching Awe, a Moral, Spiritual, and Aesthetic Emotion." Cognition and Emotion 17 (2): 297-314. https://doi.org/ 10.1080/02699930302297.

Kelty, Christopher. 2014. "Beyond Copyright and Technology: What Open Access Can Tell Us about Precarity, Authority, Innovation, and Automation in the University Today." Cultural Anthropology 29 (2): 203-215.

Kerr, Norbert L. 1998. “HARKing: Hypothesizing after the Results Are Known.” Personality and Social Psychology Review 2 (3): 196-217.

Kidwell, M.C., L.B. Lazarević, E. Baranski, T.E. Hardwicke, and S. Piechowski. 2016. “Badges to Acknowledge Open Practices: A Simple, Low-Cost, Effective Method for Increasing Transparency." PLOS Biology 14 (5): 1002456. https://doi.org/10.1371/ journal.pbio.1002456.

Kilmann, R.H., and M.J. Saxton. 1983. The Kilmann-Saxton Culture Gap Survey. Pittsburgh, PA: Organizational Design Consultants.

Kimmerer, Robin Wall. 2003. Gathering Moss: A Natural and Cultural History of Mosses. 1st ed. Corvallis, OR: Oregon State University Press.

_—_. 2013. Braiding Sweetgrass: Indigenous Wisdom, Scientific Knowledge and the Teachings of Plants. Milkweed Editions.

Kitchener, Karen Strohm, and Helene G Brenner. 1990. "Wisdom and Reflective Judgment: Knowing in the Face of Uncertainty.” Wisdom: Its Nature, Origins, and Development, 212.

Kjellström, Zakayo. 2019. "Black Open Access in Sweden : A Study on the Perceptions on and Usage of Illicit Repositories of Academic Documents.” In .

Klamer, A. 2017. Doing the Right Thing: A Value Based Economy. 2nd ed. London: Ubiquity Press. https://doi.org/10.5334/bbb.

Kleon, Austin. 2012. Steal like an Artist: 10 Things Nobody Told You about Being Creative. New York: Workman Pub. Co.

Koch, Richard, and Greg Lockwood. 2011. Superconnect: Harnessing the Power of Networks and the Strength of Weak Links. Random House Digital, Inc.

Kornfield, J. 2004. Meditation for Beginners. Boulder: Sounds True. 
Krauss, Lawrence M, and Robert H March. 1994. "Fear of Physics: A Guide for the Perplexed." Physics Today 47: 58.

Krumrei-Mancuso, Elizabeth J. 2019. "Reflections on the Science of Joy: Current Challenges and Future Directions." The Journal of Positive Psychology, October, 1-5. https://doi.org/ 10.1080/17439760.2019.1685574.

Krumrei-Mancuso, Elizabeth J., Megan C. Haggard, Jordan P. LaBouff, and Wade C. Rowatt. 2019. "Links between Intellectual Humility and Acquiring Knowledge." The Journal of Positive Psychology, February, 1-16. https://doi.org/10.1080/17439760.2019.1579359.

Krumrei-Mancuso, Elizabeth J, and Steven V Rouse. 2016. "The Development and Validation of the Comprehensive Intellectual Humility Scale." Journal of Personality Assessment 98 (2): 209-221.

Kubátová, Jaroslava, and others. 2012. “Growth of Collective Intelligence by Linking Knowledge Workers through Social Media.” LESIJ-Lex ET Scientia International Journal 19 (1): 135-145.

Kuper, Adam. 2009. Culture: The Anthropologists' Account. Harvard University Press.

Kurtz, Cynthia F, and David J Snowden. 2003. "The New Dynamics of Strategy: Sense-Making in a Complex and Complicated World." IBM Systems Journal 42 (3): 462-483.

Laloux, Frederic. 2014. Reinventing Organizations: A Guide to Creating Organizations Inspired by the next Stage in Human Consciousness. Nelson Parker.

Lancaster, Alexander K, Anne E Thessen, and Arika Virapongse. 2018. "A New Paradigm for the Scientific Enterprise: Nurturing the Ecosystem." F1000Research 7 (June): 803-803. https://doi.org/10.12688/f1000research.15078.1.

Lanier, J. 2014. Who Owns the Future? Simon and Schuster.

Larivière, V., S. Haustein, and P. Mongeon. 2015. "The Oligopoly of Academic Publishers in the Digital Era." PLoS ONE 10 (6). https://doi.org/doi:10.1371/journal.pone.0127502.

Lariviere, Vincent, and Yves Gingras. 2009. “The Impact Factor's Matthew Effect: A Natural Experiment in Bibliometrics." ArXiv:0908.3177 [Physics], August. http://arxiv.org/abs/ 0908.3177.

Larivière, Vincent, Stefanie Haustein, and Philippe Mongeon. 2015. "The Oligopoly of Academic Publishers in the Digital Era." Edited by Wolfgang Glanzel. PLOS ONE 10 (6): e0127502. https://doi.org/10.1371/journal.pone.0127502. 
Lawrence, Peter A. 2007. “The Mismeasurement of Science.” Current Biology 17 (15): R58385. https://doi.org/10.1016/j.cub.2007.06.014.

Lawson, Stuart. 2019. "Open Access Policy in the UK: From Neoliberalism to the Commons." Humanities Commons. https://doi.org/10.17613/ktz2-w249.

LeBar, Mark, ed. 2018. Justice. The Virtues: Multidisciplinary Perspectives. New York: Oxford University Press.

Ledeneva, Alena. 2018. Global Encyclopaedia of Informality, Volume 1: Towards Understanding of Social and Cultural Complexity. UCL Press.

Lehr, Jennifer K., and Ronald E. Rice. 2002. ““'Organizational Measures as a Form of Knowledge Management: A Multitheoretic, Communication-Based Exploration." Journal of the American Society for Information Science and Technology 53 (12): 1060-1073.

Lemaitre, Bruno. 2015. An Essay on Science and Narcissism: How Do High-Ego Personalities Drive Research in Life Sciences? Chicago: Federation of American Societies for Experimental Biology.

Levin, Janna. 2016. Black Hole Blues: And Other Songs from Outer Space. First edition. New York: Alfred A. Knopf.

Levine, Rick, Christopher Locke, Doc Searls, and David Weinberger. 2009. The Cluetrain Manifesto. Basic books.

Lewis, Thomas, Fari Amini, and Richard Lannon. 2001. A General Theory of Love. 1. Vintage ed. New York: Vintage.

Lindblom, Charles Edward. 1990. Inquiry and Change. Yale University Press.

Linebaugh, Peter. 2014. Stop, Thief! The Commons, Enclosures and Resistance. Oakland: PM Press.

Lynch, K, and M Ivancheva. 2016. "Academic Freedom and the Commercialisation of Universities: A Critical Ethical Analysis." Ethics in Science and Environmental Politics 15 (1): 71-85. https://doi.org/10.3354/esep00160.

MacIntyre, Alasdair C. 1984. After Virtue: A Study in Moral Theory. 2nd ed. Notre Dame, Ind: University of Notre Dame Press.

Madison, Michael J, Brett M Frischmann, and Katherine J Strandburg. 2009. "The University as Constructed Cultural Commons." Washington University Journal of Law and Policy 30: 365-403. 
Maestre, Fernando T. 2018. "Seven Steps towards Health and Happiness in the Lab." Nature, November, d41586-018-07514-17. https://doi.org/10.1038/d41586-018-07514-7.

Maintainers, The Information, D. Olson, J. Meyerson, M. A. Parsons, J. Castro, M. Lassere, D. J. Wright, et al. 2019. "Information Maintenance as a Practice of Care," June. https://doi.org/ 10.5281/zenodo.3236409.

Malone, Thomas W., Robert Laubacher, and Dellarocas Chrysanthos. 2009. Harnessing Crowds: Mapping the Genome of Collective Intelligence. MIT Sloan Research.

Malone, T.W., R. Laubacher, and C. Dellarocas. 2010. “The Collective Intelligence Genome.” MIT Sloan Management Review 51 (3): 21.

Manuel, Frank Edward. 1979. A Portrait of Isaac Newton. Washington: New Republic Books.

Markus, Hazel Rose, and Alana Conner. 2014. Clash!: How to Thrive in a Multicultural World. Penguin.

Martin, Ronald E. 1998. One Long Experiment: Scale and Process in Earth History. Columbia University Press.

Massy, William F. 2016. Reengineering the University: How to Be Mission Centered, Market Smart, and Margin Conscious. JHU Press.

McCarthy, Michael. 2015. The Moth Snowstorm: Nature and Joy. New York Review of Books.

McGinnis, M.D. 2011. "An Introduction to IAD and the Language of the Ostrom Workshop: A Simple Guide to a Complex Framework." Policy Studies Journal 39 (1): 169-183.

McKiernan, E.C., P.E. Bourne, C.T. Brown, S. Buck, A. Kenall, J. Lin, D. McDougall, et al. 2016. How Open Science Helps Researchers Succeed.

McKiernan, Erin C., Lesley A. Schimanski, Carol Muñoz Nieves, Lisa Matthias, Meredith T. Niles, and Juan Pablo Alperin. 2019. "Use of the Journal Impact Factor in Academic Review, Promotion, and Tenure Evaluations." PeerJ Preprints 7 (April): e27638v2. https://doi.org/ 10.7287/peerj.preprints.27638v2.

McMillan, D.W., and M. D. Chavis. 1986. "Sense of Community: A Definition and Theory." J. Community Psychol 14: 6-23. https://doi.org/ doi:10.1002/1520-6629(198601)14:1<6::AID-JCOP2290140103>3.0.CO;2-I.

McPhetres, Jonathon. 2019. “Oh, the Things You Don't Know: Awe Promotes Awareness of Knowledge Gaps and Science Interest." Cognition and Emotion, February, 1-17. https:// doi.org/10.1080/02699931.2019.1585331. 
Meindl, Peter, Eranda Jayawickreme, R. Michael Furr, and William Fleeson. 2015. “A Foundation Beam for Studying Morality from a Personological Point of View: Are Individual Differences in Moral Behaviors and Thoughts Consistent?" Journal of Research in Personality 59 (December): 81-92. https://doi.org/10.1016/j.jrp.2015.09.005.

Merchant, Nilofer. 2017. The Power of Onlyness: Make Your Wild Ideas Mighty Enough to Dent the World. New York, New York: Viking.

Merton, Robert K. 1942. "Science and Technology in a Democratic Order." Journal of Legal and Political Sociology 1 (1): 115-126.

- - - 1973. The Sociology of Science: Theoretical and Empirical Investigations. University of Chicago press.

- - 1 1988. "The Matthew Effect in Science, II: Cumulative Advantage and the Symbolism of Intellectual Property." Isis 79 (4): 606-623.

Meyerhoff, Eli, Elizabeth Johnson, and Bruce Braun. 2011. "Time and the University." ACME: An International e-Journal for Critical Geographies. 10 (3): 483-507.

Millman, D. 2007. How to Think like a Great Graphic Designer. Skyhorse Publishing Inc.

Mitchell Jr, Richard G. 1992. "Sociological Implications of the Flow Experience."

Montgomery, L., J. Hartley, C. Neylon, M. Gillies, E. Gray, C. Herrmann-Pillath, and K. Wilson. 2018. Open Knowledge Institutions. MIT Press OA Books. https://doi.org/ 10.21428/99f89a34/a594a484.

Moore, S., C. Neylon, M.P. Eve, D.P. O’Donnell, and D Pattinson. 2017. “'Excellence R Us': University Research and the Fetishisation of Excellence." Palgrave Communications 3: 16105.

Morrison, Toni. 2019. The Source of Self-Regard: Selected Essays, Speeches, and Meditations. Knopf.

Moshagen, Morten, Benjamin E. Hilbig, and Ingo Zettler. 2018. "The Dark Core of Personality." Psychological Review 125 (5): 656-88. https://doi.org/10.1037/rev0000111.

Mountz, Alison, Anne Bonds, Becky Mansfield, Jenna Loyd, Jennifer Hyndman, Margaret Walton-Roberts, Ranu Basu, et al. 2015. "For Slow Scholarship: A Feminist Politics of Resistance through Collective Action in the Neoliberal University." ACME: An International E-Journal for Critical Geographies 14 (4). 
Mowery, David C, Richard R Nelson, Bhaven N Sampat, and Arvids A Ziedonis. 2001. "The Growth of Patenting and Licensing by US Universities: An Assessment of the Effects of the Bayh-Dole Act of 1980." Research Policy 30 (1): 99-119.

Mozilla Foundation, The, and Peer 2 Peer University. 2012. “Open Badges for Lifelong Learning:Exploring an Open Badge Ecosystem to Support Skill Development and Lifelong Learning for Real Results Such as Jobs and Advancement" Working Paper, Funding from The MacArthur Foundation.

Muller, Jerry Z. 2018. The Tyranny of Metrics. Princeton Oxford: Princeton University Press.

Münch, Richard. 2015. "Science in the Hands of Strategic Management: The Metrification of Scientific Work and Its Impact on the Evolution of Knowledge." In Incentives and Performance, edited by Isabell M. Welpe, Jutta Wollersheim, Stefanie Ringelhan, and Margit Osterloh, 33-48. Cham: Springer International Publishing. https://doi.org/ 10.1007/978-3-319-09785-5_3.

Napolitano, Christopher M. 2013. "More than Just a Simple Twist of Fate: Serendipitous Relations in Developmental Science." Human Development 56 (5): 291-318. https:// doi.org/10.1159/000355022.

National Academies of Sciences, Engineering, and Medicine. 2020. Promising Practices for Addressing the Underrepresentation of Women in Science, Engineering, and Medicine: Opening Doors. Edited by Rita Colwell, Ashley Bear, and Alex Helman. Washington, D.C.: National Academies Press. https://doi.org/10.17226/25585.

National Academies of Sciences, Engineering, and Medicine, NAS Committee on the Impacts of Sexual Harassment in Academia, Committee on Women in Science, Engineering, and Medicine, and Policy and Global Affairs. 2018. Sexual Harassment of Women: Climate, Culture, and Consequences in Academic Sciences, Engineering, and Medicine. Edited by Paula A. Johnson, Sheila E. Widnall, and Frazier F. Benya. Washington, D.C.: National Academies Press. https://doi.org/10.17226/24994.

National Academies of Sciences, Engineering, and Medicine (U.S.). 2018. Open Science by Design: Realizing a Vision for 21st Century Research. A Consensus Study Report. Washington, DC: The National Academies Press.

Newfield, Christopher. 2016. The Great Mistake: How We Wrecked Public Universities and How We Can Fix Them. JHU Press.

Neylon, Cameron. 2017a. "Openness in Scholarship: A Return to Core Values?" Stand Alone, 6-17. https://doi.org/10.3233/978-1-61499-769-6-6. 
_- - 2017b. "Sustaining Scholarly Infrastructures through Collective Action: The Lessons That Olson Can Teach Us.” Preprint. Scientific Communication and Education. https:// doi.org/10.1101/116756.

Neylon, Cameron, Rene Belsø, Magchiel Bijsterbosch, Bas Cordewener, Jérôme Foncel, Sascha Friesike, Aileen Fyfe, et al. 2019. Open Scholarship and the Need for Collective Action. Zenodo. https://doi.org/10.5281/ZENODO.3454688.

Nielsen, M. 2011. Reinventing Discovery: The New Era of Networked Science. Princeton University Press.

Nixon, Natalie W. 2020. The Creativity Leap: Unleash Curiosity, Improvisation, and Intuition at Work. First edition. Oakland, CA: Berrett-Koehler Publishers, Inc.

Noddings, Nel. 1988. "An Ethic of Caring and Its Implications for Instructional Arrangements." American Journal of Education 96 (2): 215-230.

——_. 2003. Happiness and Education. Cambridge University Press.

Nordhoff, Sebastian. 2018. Cookbook for Open Access Books. Language Science Press, Berlin. https://doi.org/10.5281/zenodo.1286925.

Norton, Michael I., Daniel Mochon, and Dan Ariely. 2012. "The IKEA Effect: When Labor Leads to Love." Journal of Consumer Psychology 22 (3): 453-60. https://doi.org/10.1016/ j.jcps.2011.08.002.

Nosek, Brian A, Jeffrey R Spies, and Matt Motyl. 2012. "Scientific Utopia: II. Restructuring Incentives and Practices to Promote Truth over Publishability." Perspectives on Psychological Science 7 (6): 615-631.

Nussbaum, Martha C. 1995. “Emotions and Women's Capabilities.” Women, Culture and Development: A Study of Human Capabilities, 360-395.

O’Keefe, Paul A, Carol S Dweck, and Gregory M Walton. 2018. "Implicit Theories of Interest: Finding Your Passion or Developing It?” Psychological Science 29 (10): 1653-1664.

Olson, Randy. 2015. Houston, We Have a Narrative: Why Science Needs Story. University of Chicago Press.

——_. 2019. Narrative Is Everything: The ABT Framework and Narrative Evolution.

Osborne, Robin. 2013. “Why Open Access Makes No Sense.” Debating Open Access 10.

Osterloh, Margit, and Bruno S. Frey. 1999. "Motivation, Knowledge Transfer, and Organizational Form.” ETH Zurich. https://doi.org/10.3929/ethz-a-004373006. 
Osterloh, Margit, and Bruno S Frey. 2011. "Rankings Games." University of Zurich. https:// doi.org/10.5167/uzh-51543.

Ostrom, Elinor. 2015. Governing the Commons. Cambridge university press.

Ouchi, W.G. 1981. Theory Z. Reading, MA: Addison-Wesley.

Overall, Patricia Montiel. 2009. "Cultural Competence: A Conceptual Framework for Library and Information Science Professionals." The Library Quarterly 79 (2): 175-204.

P2P Foundation, GB, Michel Bauwens, Vasilis Kostakis, Tallinn University of Technology; P2P Lab, EE, Alex Pazaitis, and Tallinn University of Technology; P2P Lab, EE. 2019. Peer to Peer: The Commons Manifesto. University of Westminster Press. https://doi.org/10.16997/ book33.

Packard, D. 1995. The HP Way. New York: HarperCollins.

Pagoto, Sherry, and Camille Nebeker. 2019. "How Scientists Can Take the Lead in Establishing Ethical Practices for Social Media Research." https://doi.org/10.1093/jamia/ ocy174.

Panitch, J.M., and S. Michalak. 2015. The Serials Crisis: A White Paper for the UNC-Chapel Hill Scholarly Communications Convocation. Online.

Pascale, R.T., and A.G. Athos. 1981. The Art of Japanese Management. New York: Simon \& Schuster.

Peters, T.J., and R.H. Waterman Jr. 1982. In Search of Excellence. New York: HarperCollins.

Peterson, N. 1993. "Demand Sharing: Reciprocity and the Pressure for Generosity among Foragers." American Anthropologist 95 (4): 860-874.

Phillips, Adam, and Barbara Taylor. 2009. On Kindness. 1st American ed. New York: Farrar, Straus and Giroux.

Planck, Max, Albert Einstein, and James Murphy. 1932. Where Is Science Going?

Pluchino, Alessandro, Alessio Emanuele Biondo, and Andrea Rapisarda. 2018. "TALENT VERSUS LUCK: THE ROLE OF RANDOMNESS IN SUCCESS AND FAILURE." Advances in Complex Systems 21 (03n04): 1850014. https://doi.org/10.1142/S0219525918500145.

Polanyi, M. 1962. "The Republic of Science: Its Political and Economic Theory." Minerva 1: 54-73. 
Pondy, L.R., P.J. Frost, G. Morgan, and T. Dandridge, eds. 1983. Organizational Symbolism. Greenwich, CT: JAI Press.

Potts, J., J. Hartley, L. Montgomery, C. Neylon, and E. Rennie. 2017. “A Journal Is a Club: A New Economic Model for Scholarly Publishing." Prometheus 35 (1): 75-92. https://doi.org/ DOI: $10.1080 / 08109028.2017 .1386949$.

Pusey, Andre. 2017. "Towards a University of the Common: Reimagining the University in Order to Abolish It with the Really Open University." Open Library of Humanities 3 (2): 1. https://doi.org/10.16995/olh.90.

Putnam, R.D. 2000. "Bowling alone: America's declining social capital." In Culture and politics, 223-234. New York: Palgrave Macmillan.

Rathunde, Kevin. 2010. "Experiential Wisdom and Optimal Experience: Interviews with Three Distinguished Lifelong Learners." Journal of Adult Development 17 (2): 81-93. https://doi.org/10.1007/s10804-009-9083-x.

- - 2015. "Creating a Context for Flow: The Importance of Personal Insight and Experience." NAMTA Journal 40 (3): 15-27.

Rheingold, H. 2002. Smart mobs: The next social revolution. Publisher: Basic Books.

Rice, Ronald E, and Stephen D Cooper. 2010. Organizations and Unusual Routines: A Systems Analysis of Dysfunctional Feedback Processes. Cambridge University Press.

Ridley, M. 2010. The Rational Optimist: How Prosperity Evolves. 4th Estate.

Ritti, R Richard, and G Ray Funkhouser. 1982. "The Ropes to Skip and the Ropes to Know. Columbus." Ohio: Grid.

Roberts, Brent W., M. Brent Donnellan, and Patrick L. Hill. 2012. "Personality Trait Development in Adulthood." In Handbook of Psychology, Second Edition, edited by Irving Weiner, hop205009. Hoboken, NJ, USA: John Wiley \& Sons, Inc. https://doi.org/ 10.1002/9781118133880.hop205009.

Roberts, Royston M. 1989. Serendipity: Accidental Discoveries in Science. Wiley Science Editions. New York: Wiley.

Rogers, Carl Ransom. 1995. On Becoming a Person: A Therapist's View of Psychotherapy. Houghton Mifflin Harcourt.

Rowland, Stephen. 2008. “Collegiality and Intellectual Love.” British Journal of Sociology of Education 29 (3): 353-60. https://doi.org/10.1080/01425690801966493. 
- - 2009. "Kindness." London Review of Education 7 (3): 207-210.

Ruef, Martin. 2002. "Strong Ties, Weak Ties, and Islands: Structural and Cultural Predictors of Organizational Innovation." Industrial and Corporate Change 11 (3): 427-449.

Sagan, Carl. 1987. "The Burden of Skepticism." Skeptical Inquirer 12 (1): 38-46.

Sahlins, Marshall David. 2011. Stone Age Economics. New Brunswick, NJ: Transaction Publishers.

Saint-Exupéry, Antoine de. 1948. Citadelle. Gallimard,.

Sargut, G., and R.G. McGrath. 2011. "Learning to Live with Complexity." Harvard Business Review 89 (9): 68-76.

Scardamalia, Marlene. 2002. "Collective Cognitive Responsibility for the Advancement of Knowledge." Liberal Education in a Knowledge Society 97: 67-98.

Schein, Edgar H. 2010. Organizational Culture and Leadership. Vol. 2. John Wiley \& Sons.

_-_. 2013. Humble Inquiry: The Gentle Art of Asking Instead of Telling. Berrett-Koehler Publishers.

Schein, E.H. 1968. "Organizational socialization and the profession of management." Industrial Management Review 9: 1-15.

- - - 1978. Career Dynamics: Matching Individual and Organizational Needs. Reading, MA: Addison-Wesley.

Schmidt, E., and J. Rosenberg. 2014. How google works. Hachette UK.

Schneider, B., ed. 1990. Organizational Climate and Culture. San Francisco, CA: Jossey-Bass.

Scholz, Trebor, and Nathan Schneider. 2017. Ours to Hack and to Own: The Rise of Platform Cooperativism, a New Vision for the Future of Work and a Fairer Internet. OR books.

Schuler, D. 2008. Liberating Voices: A Pattern Language for Communication Revolution. MIT Press.

Schultz, M. 1995. On Studying Organizational Cultures. New York: Walter de Gruyter.

Schultz, Vicki. 2002. “The Sanitized Workplace.” Yale Lj 112: 2061.

Schulz, Kathryn. 2011. Being wrong: Adventures in the margin of error. Granta Books.

Schwartz, Barry, and Kenneth Sharpe. 2010. Practical Wisdom: The Right Way to Do the Right Thing. Penguin. 
Schwartz, Barry, and Kenneth E. Sharpe. 2006. "Practical Wisdom: Aristotle Meets Positive Psychology." Journal of Happiness Studies 7 (3): 377-95. https://doi.org/10.1007/ s10902-005-3651-y.

Scotchmer, Suzanne. 1991. "Standing on the Shoulders of Giants: Cumulative Research and the Patent Law." Journal of Economic Perspectives 5 (1): 29-41. https://doi.org/10.1257/ jep.5.1.29.

Seligman, Martin EP, and Mihaly Csikszentmihalyi. 2014. "Positive Psychology: An Introduction." In Flow and the Foundations of Positive Psychology, 279-298. Springer.

Senge, P.M. 1990. The Fifth Discipline. New York: Doubleday Currency.

Sharma, Ankita, and Roshan Lal Dewangan. 2017. "Can Wisdom Be Fostered: Time to Test the Model of Wisdom." Edited by Feng Kong. Cogent Psychology 4 (1). https://doi.org/ $10.1080 / 23311908.2017 .1381456$.

Sharpe, Kenneth, and Elizabeth Bolton. 2016. "Teaching Ourselves to Teach.” Inside Higher Ed.

Shirky, C. 2010. Cognitive Surplus: Creativity and Generosity in a Connected Age. Penguin UK.

Shirky, Clay. 2008. Here Comes Everybody: The Power of Organizing without Organizations. Penguin.

Siefkes, C. 2008. From Exchange to Contributions: Generalizing Peer Production into the Physical World. Berlin: Edition.

Sigl, Lisa, Ulrike Felt, and Maximilian Fochler. 2020. “'I Am Primarily Paid for Publishing...' The Narrative Framing of Societal Responsibilities in Academic Life Science Research." Science and Engineering Ethics 26 (3): 1569-93. https://doi.org/10.1007/ s11948-020-00191-8.

Sinek, Simon. 2009. Start with Why: How Great Leaders Inspire Everyone to Take Action. Penguin Group. Kindle Edition.

___ 2019. The Infinite Game. New York: Portfolio/Penguin.

Skinner, Quentin. 1969. "II. Thomas Hobbes and the Nature of the Early Royal Society." The Historical Journal 12 (02): 217. https://doi.org/10.1017/S0018246X00004271.

Slaughter, Sheila, and Larry L. Leslie. 2001. "Expanding and Elaborating the Concept of Academic Capitalism.” Organization 8 (2): 154-61. https://doi.org/ 10.1177/1350508401082003. 
Slaughter, Sheila, and Gary Rhoades. 2010. Academic Capitalism and the New Economy: Markets, State, and Higher Education. Paperback ed. Baltimore: Johns Hopkins Univ. Press.

Smaldino, Paul E, and Richard McElreath. 2016. "The Natural Selection of Bad Science." Royal Society Open Science 3 (9): 160384.

Smaldino, Paul, Matthew Adam Turner, and Pablo Andrés Contreras Kallens. 2019. "Open Science and Modified Funding Lotteries Can Impede the Natural Selection of Bad Science." Preprint. Open Science Framework. https://doi.org/10.31219/osf.io/zvkwq.

Smichowski, Bruno Carballa. 2016. "Data as a Common in the Sharing Economy: A General Policy Proposal." https://hal.archives-ouvertes.fr/hal-01386644.

Smircich, L. 1983. "Concepts of Culture and Organizational Analysis." Administrative Science Quarterly 28: 339-358.

Smith, G. 2009. Democratic Innovations: Designing Institutions for Citizen Participation. Cambridge: Cambridge University Press.

Smith, M.K. 2001. "Chris Argyris: Theories of Action, Double-Loop Learning and Organizational Learning," The Encyclopedia of Informal Education, Http://Www.Infed.Org/ Thinkers/Argyris.Htm. Last Update.

Snowden, David. 2000. "Organic Knowledge Management: Part I The ASHEN Model: An Enabler of Action." Knowledge Management 3 (7): 14-17.

-_- 2002. "Complex Acts of Knowing: Paradox and Descriptive Self-Awareness." Journal of Knowledge Management 6 (2): 100-111.

Snowden, David J, and Mary E Boone. 2007. "A Leader's Framework for Decision Making." Harvard Business Review 85 (11): 68.

Soderqvist, Thomas. 2008. Science as Autobiography: The Troubled Life of Niels Jern. Yale University Press.

Sosa, Ernest. 2015. Judgment and Agency. Oxford University Press. https://doi.org/ 10.1093/acprof:oso/9780198719694.001.0001.

Standing, Guy. 2016. The Precariat: The New Dangerous Class. Revised edition. London ; New York: Bloomsbury Academic, an imprint of Bloomsbury Publishing Plc.

Sternberg, Robert J. 1990. Wisdom: Its Nature, Origins, and Development. Cambridge University Press. 
Stichter, Matt. 2007. "Ethical Expertise: The Skill Model of Virtue." Ethical Theory and Moral Practice 10 (2): 183-94. https://doi.org/10.1007/s10677-006-9054-2.

Stodden, Victoria. 2014. "Intellectual Property and Computational Science." In Opening Science, edited by Sönke Bartling and Sascha Friesike, 225-35. Cham: Springer International Publishing. https://doi.org/10.1007/978-3-319-00026-8_15.

Strandburg, Katherine J. 2005. "Curiosity-Driven Research and University Technology Transfer." University Entrepreneurship and Technology Transfer: Process, Design, and Intellectual Property, 93-123.

Strathern, M. 2002. Abstraction and Decontextualization: An Anthropological Comment. Virtual Society: Technology, Cyberbole, Reality.

Suber, P. 2012. Open Access. MIT Press.

Sutton, R.I. 2007. The No Asshole Rule: Building a Civilized Workplace and Surviving One That Isn't. Hachette UK.

Suzman, J. 2017a. Affluence Without Abundance: The Disappearing World of the Bushmen. Bloomsbury Publishing USA.

-_- 2017b. "Why 'Bushman Banter' Was Crucial to Hunter-Gatherers' Evolutionary Success." The Guardian October 29.

Tagiuri, R., and G.H. Litwin. 1968. "Organizational Climate: Exploration of a Concept.” Division of Research, Harvard Graduate School of Business.

Taleb, N.N. 2012. Antifragile: Things That Gain from Disorder (Vol. 3). Random House Incorporated.

Tangney, June Price. 2000. "Humility: Theoretical Perspectives, Empirical Findings and Directions for Future Research." Journal of Social and Clinical Psychology 19 (1): 70-82.

Tanner, K.J. 2005. "Emotion, Gender and the Sustainability of Communities." The Journal of Community Informatics 1 (2).

Tcherneva, Pavlina R. 2020. The Case for a Job Guarantee. S.l.: Polity Press.

Tennant, Jonathan. 2019. Supplemental Materials for Preprint: Foundations for Open Scholarship Strategy Development. OSF. osf.io/wbfru.

Tennant, Jonathan, Natalia Z Bielczyk, Bastian Greshake Tzovaras, Paola Masuzzo, and Tobias Steiner. 2019. “Introducing Massively Open Online Papers (MOOPs).” Preprint. MetaArXiv. https://doi.org/10.31222/osf.io/et8ak. 
Tennant, Jonathan P., Harry Crane, Tom Crick, Jacinto Davila, Asura Enkhbayar, Johanna Havemann, Bianca Kramer, et al. 2019. "Ten Hot Topics around Scholarly Publishing." Publications 7 (2): 34. https://doi.org/10.3390/publications7020034.

Tennant, Jonathan P, Harry Crane, Tom Crick, Jacinto Davila, Asura Enkhbayar, Johanna Havemann, Bianca Kramer, et al. n.d. "Ten Myths around Open Scholarly Publishing." Accessed March 12, 2019. https://doi.org/10.7287/peerj.preprints.27580v1.

Tennant, Jonathan P., Jonathan M. Dugan, Daniel Graziotin, Damien C. Jacques, François Waldner, Daniel Mietchen, Yehia Elkhatib, et al. 2017. "A Multi-Disciplinary Perspective on Emergent and Future Innovations in Peer Review." F1000Research 6 (November): 1151. https://doi.org/10.12688/f1000research.12037.3.

Tennant, Jonathan, and Tony Ross-Hellauer. 2019. "The Limitations to Our Understanding of Peer Review." Preprint. SocArXiv. https://doi.org/10.31235/osf.io/jq623.

Tennant, Jonathan, Ritwik Agarwal, Ksenija Baždarić, David Brassard, Tom Crick, Daniel J. Dunleavy, Thomas R. Evans, et al. 2020. "A Tale of Two 'opens': Intersections Between Free and Open Source Software and Open Scholarship." SocArXiv. March 6. doi:10.31235/osf.io/ $2 \mathrm{kxq} 8$.

Thomas, Douglas, and John Seely Brown. 2007. "The Play of Imagination: Extending the Literary Mind." Games and Culture 2 (2): 149-72. https://doi.org/ $10.1177 / 1555412007299458$.

- - 2 2011. A New Culture of Learning: Cultivating the Imagination for a World of Constant Change. Vol. 219. CreateSpace Lexington, KY.

Thompson, E.P. 2015. Customs in Common: Studies in Traditional Popular Culture. New: Press, The.

Thompson, Hunter S. 1972. Fear and Loathing in Las Vegas; a Savage Journey to the Heart of the American Dream. Random House.

Trice, H.M., and J.M. Beyer. 1985. "Using Six Organizational Rites to Change Culture." In , edited by R.H. Kilmann, M.J. Saxton, R. Serpa, and and, 370-399. San Francisco, CA: JosseyBass.

1993. The Cultures of Work Organizations. Englewood Cliffs, NJ: Prentice Hall.

Tsai, Cheng-hung. 2019. "Phronesis and Techne: The Skill Model of Wisdom Defended." Australasian Journal of Philosophy, June, 1-14. https://doi.org/

10.1080/00048402.2019.1618352. 
Twenge, J.M., and W. K. Campbell. 2009. The Narcissism Epidemic: Living in the Age of Entitlement. Simon and Schuster.

Ullmann-Margalit, Edna. 2011. "Considerateness." Iyyun: The Jerusalem Philosophical Quarterly/עיון: רבעון פילוסופי 205-244.

University of Virginia \& Center for Open Science, and Brian A. Nosek. 2017. "Opening Science." In Open: The Philosophy and Practices That Are Revolutionizing Education and Science, edited by Kwantlen Polytechnic University, CA, Rajiv S. Jhangiani, Robert BiswasDiener, and Noba Project, 89-99. Ubiquity Press. https://doi.org/10.5334/bbc.g.

“Valve Corporation Handbook for New Employees." 2012. http://newcdn.flamehaus.com/ Valve_Handbook_LowRes.pdf.

Van de Ven, Andrew H. 1986. "Central Problems in the Management of Innovation." Management Science 32 (5): 590-607.

Van Maanen, J. 1979a. "The Fact of Fiction in Organizational Ethnography." Administrative Science Quarterly 24: 539-550.

_- - 1979b. "The Self, the Situation, and the Rules of Interpersonal Relations." In , edited by W.Bennis and. Florence, KY: Dorsey Press.

Van Maanen, J., and S.R. Barley. 1984. "Occupational Communities: Culture and Control in Organizations." In Research in Organizational Behavior, edited by B.M. Staw and L.L. Cummings. Vol. 6. Greenwich, CT: JAI Press.

Velleman, J David. 2006. Self to Self: Selected Essays. Cambridge University Press.

Vincent, Nigel, and Chris Wickham. 2013. "Debating Open Access." In . British Academy.

Wahls, Wayne P. 2018. "The NIH Must Reduce Disparities in Funding to Maximize Its Return on Investments from Taxpayers." ELife 7 (March). https://doi.org/10.7554/eLife.34965.

Washburn, Jennifer. 2008. University, Inc.: The Corporate Corruption of Higher Education. Basic Books.

Way, Samuel F., Allison C. Morgan, Daniel B. Larremore, and Aaron Clauset. 2019. "Productivity, Prominence, and the Effects of Academic Environment." Proceedings of the National Academy of Sciences 116 (22): 10729-33. https://doi.org/10.1073/ pnas.1817431116.

Wayment, Heidi A., Jack J. Bauer, and Kateryna Sylaska. 2015. "The Quiet Ego Scale: Measuring the Compassionate Self-Identity." Journal of Happiness Studies 16 (4): 999-1033. https://doi.org/10.1007/s10902-014-9546-z. 
Weick, K. 1995. Sense-making in organizations. Thousand Oaks. CA: Sage.

Weinberger, David. 2014. Too Big to Know: Rethinking Knowledge Now That the Facts Aren't the Facts, Experts Are Everywhere, and the Smartest Person in the Room Is the Room.

Welpe, Isabell M., Jutta Wollersheim, Stefanie Ringelhan, and Margit Osterloh, eds. 2015. Incentives and Performance. Cham: Springer International Publishing. https://doi.org/ 10.1007/978-3-319-09785-5.

Wenger, E., R.A. McDermott, and W. Snyder. 2002. Cultivating Communities of Practice: A Guide to Managing Knowledge. Harvard Business Press.

Whitchurch, Celia. 2013. Reconstructing Identities in Higher Education: The Rise of "Third Space" Professionals. Research into Higher Education. New York, NY: Routledge.

2015. "The Rise of Third Space Professionals: Paradoxes and Dilemmas." In Forming, Recruiting and Managing the Academic Profession, edited by Ulrich Teichler and William K. Cummings, 79-99. Cham: Springer International Publishing. https://doi.org/ 10.1007/978-3-319-16080-1_5.

Widlok, Thomas. 2013. “Sharing: Allowing Others to Take What Is Valued.” HAU: Journal of Ethnographic Theory 3 (2): 11-31.

—_- 2016. Anthropology and the Economy of Sharing. Routledge.

_- _ 2018. Learning How to Share. Vol. 1. Oxford University Press. https://doi.org/ 10.1093/oso/9780190631741.003.0005.

Willis, Paul. 2016. "From Humble Inquiry to Humble Intelligence: Confronting Wicked Problems and Augmenting Public Relations." Public Relations Review 42 (2): 306-313.

Wilson, Greg. 2020. Teaching Tech Together: How to Create and Deliver Lessons That Work and Build a Teaching Community around Them. Boca Raton: CRC Press.

Wiseman, Liz. 2017. Multipliers: How the Best Leaders Make Everyone Smarter. Revised and Updated edition. New York, NY: HarperBusiness, an imprint of HarperCollinsPublishers.

Wolpe, Paul Root. 2006. "Reasons Scientists Avoid Thinking about Ethics." Cell 125 (6): 1023-25. https://doi.org/10.1016/j.cell.2006.06.001.

Woolgar, Steve, ed. 2002. Virtual Society? Technology, Cyberbole, Reality. Oxford ; New York: Oxford University Press.

Yaqub, Ohid. 2018. "Serendipity: Towards a Taxonomy and a Theory." Research Policy 47 (1): 169-79. https://doi.org/10.1016/j.respol.2017.10.007. 
Yunkaporta, Tyson. 2020. Sand Talk: How Indigenous Thinking Can Save the World. First edition. New York, New York: HarperOne, an imprint of HarperCollins Publications.

Zachry, Corinne E, Le Vy Phan, Laura E R Blackie, and Eranda Jayawickreme. 2018. "Situation-Based Contingencies Underlying Wisdom-Content Manifestations: Examining Intellectual Humility in Daily Life." The Journals of Gerontology: Series B 73 (8): 1404-15. https://doi.org/10.1093/geronb/gby016.

Zalta, Edward N, Uri Nodelman, Colin Allen, and R Lanier Anderson. 2002. "Stanford Encyclopedia of Philosophy." See Http://Plato. Stanford. Edu/. Received September.

Ziman, John. 2002. Real Science: What It Is and What It Means. Cambridge University Press.

Zittrain, Jonathan. 2008. The Future of the Internet-and How to Stop It. Yale University Press.

Zwanenberg, Patrick van, Mariano Fressoli, Valeria Arza, Adrian Smith, and Anabel Marin. 2017. "Open and Collaborative Developments." 